2012

\title{
Torque converter turbine noise and cavitation noise over varying speed ratio
}

Chad Michael Walber

Michigan Technological University

Copyright 2012 Chad Michael Walber

\section{Recommended Citation}

Walber, Chad Michael, "Torque converter turbine noise and cavitation noise over varying speed ratio", Dissertation, Michigan Technological University, 2012.

https://digitalcommons.mtu.edu/etds/420

Follow this and additional works at: https://digitalcommons.mtu.edu/etds

Part of the Mechanical Engineering Commons 


\title{
TORQUE CONVERTER TURBINE NOISE AND CAVITATION NOISE OVER VARYING SPEED RATIO
}

By

Chad Michael Walber

\author{
A DISSERATION \\ Submitted in partial fulfillment of the requirements for the degree of \\ DOCTOR OF PHILOSOPHY \\ (Mechanical Engineering-Engineering Mechanics) \\ MICHIGAN TECHNOLOGICAL UNIVERSITY \\ 2012 \\ (C) 2012 Chad Michael Walber
}



This dissertation, "Torque Converter Turbine Noise and Cavitation Noise over Varying Speed Ratio," is hereby approved in partial fulfillment of the requirements for the degree of DOCTOR OF PHILOSOPHY IN MECHANICAL ENGINEERING-ENGINEERING MECHANICS.

Department of Mechanical Engineering-Engineering Mechanics

Signatures:

Dissertation Advisor

Jason R. Blough

Department Chair

William W. Predebon

Date 



\section{Table of Contents}

List of Figures........................................................................................................................ vii

List of Tables ........................................................................................................................... xiii

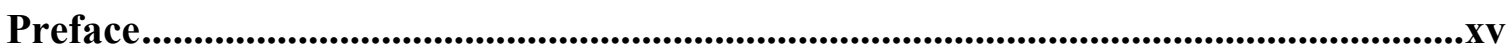

Acknowledgements ............................................................................................................. xvii

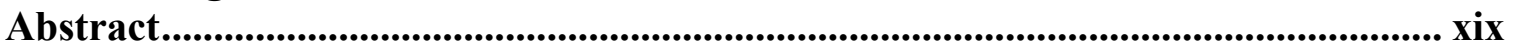

Chapter 1 - Literature Review ................................................................................................1

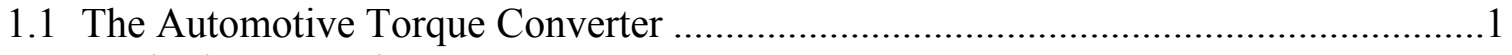

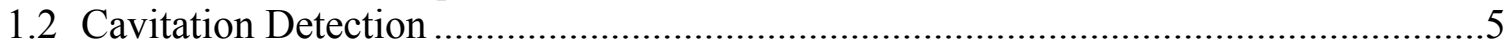

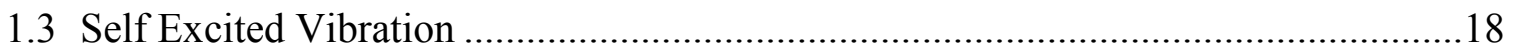

1.4 Torque Converter Turbine Noise and Cavitation Noise over Varying Speed Ratio ..26

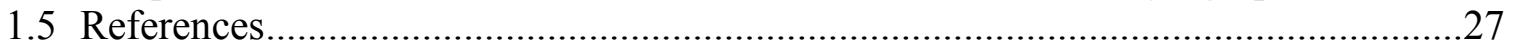

Chapter 2 - Predicting Cavitation Desinence in Automotive Torque Converters ....31

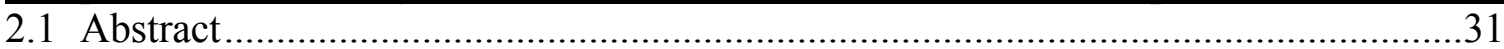

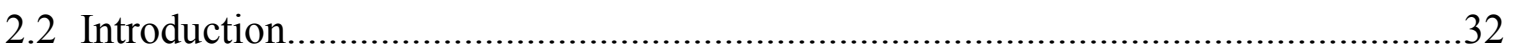

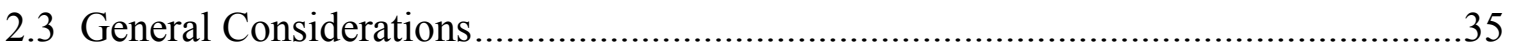

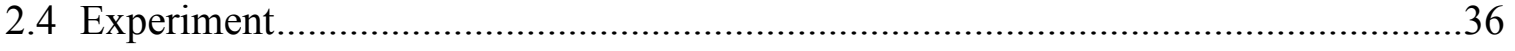

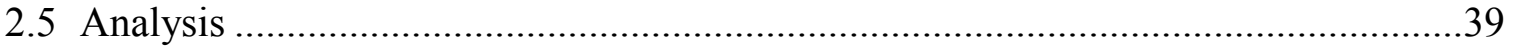

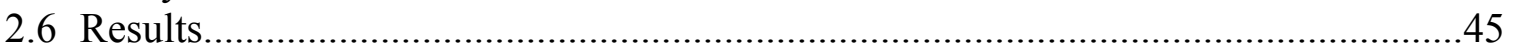

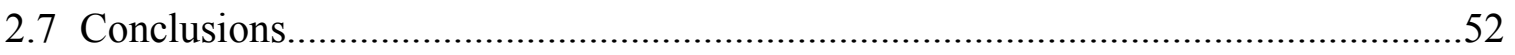

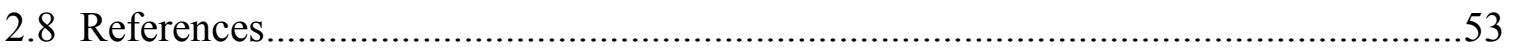

Chapter 3 - Measuring and Comparing Frequency Response Functions of Torque Converter Turbines Submerged in Transmission Fluid ....................................................55

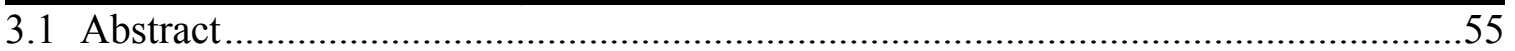

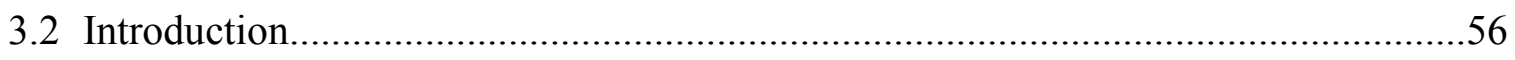

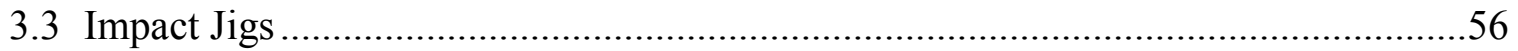

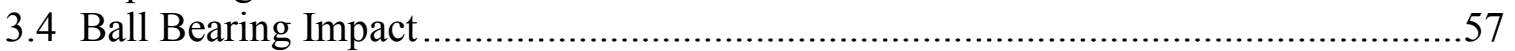

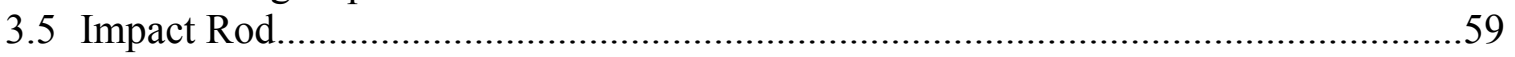

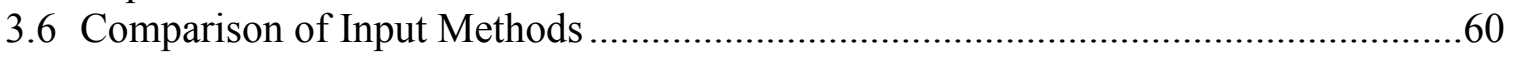

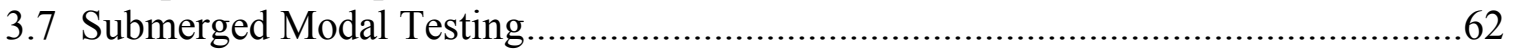

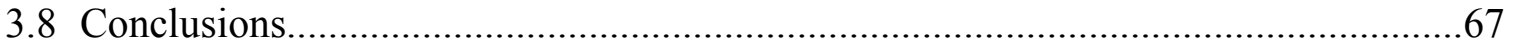

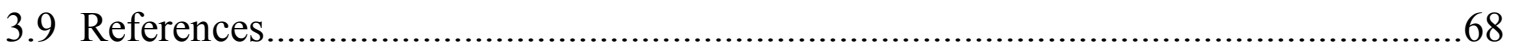

Chapter 4-Characterizing Torque Converter Turbine Noise ....................................69

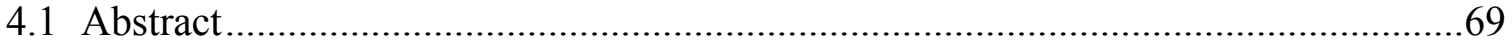

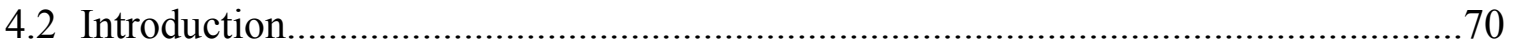

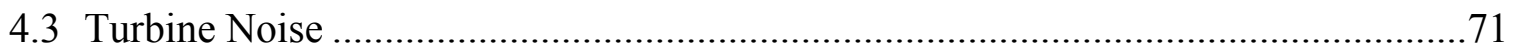

4.4 Vortex Shedding and Vortex-induced Vibration ......................................................

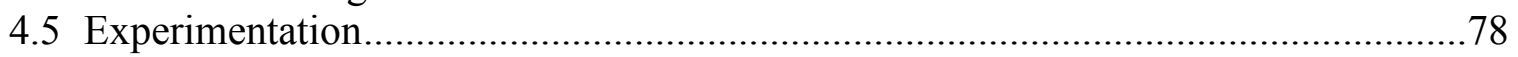

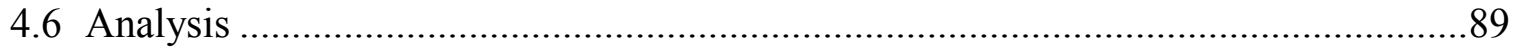

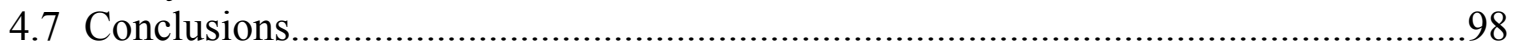




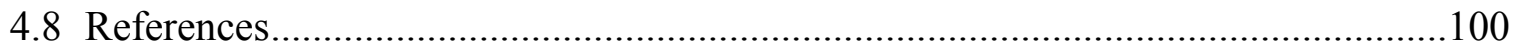

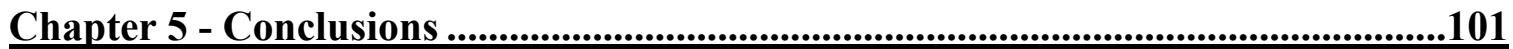

5.1 Experimental Desinent Cavitation Conclusions ..................................................101

5.2 Turbine Noise Study Conclusions ...................................................................... 102

5.3 General Automotive Torque Converter Recommendations .................................102

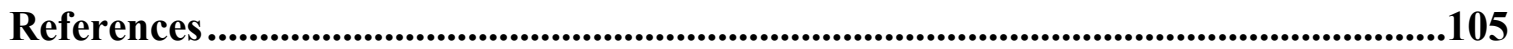

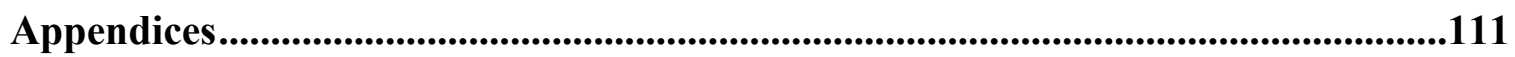




\section{List of Figures}

\section{Chapter 1 - Literature Review}

Figure 1.1: Cross section of an automotive torque converter; toroidal flow is indicated by the direction of the arrows with no tail. Arrows with tails indicate the normal cooling flow. (1) 2

Figure 1.2: Flow incidence angle across the blades of the stator. (1) 3

Figure 1.3: Left: Pressure - Volume phase diagram depicting difference between boiling (constant arrow) and cavitation (dashed arrow). Right: Pressure - Temperature phase diagram depicting difference between boiling (constant arrow) and cavitation (dashed arrow). 6

Figure 1.4: Submarine noise showing transition to noisy operation (cavitation) to depend on depth. (1) 10

Figure 1.5: Effect of charge pressure on the onset of cavitation for a sample torque converter. (1)

Figure 1.6: Left: Comparison of original torque converter dynamometer test fixture (Red) and acoustic test fixture (Gray). Right: Close up of opened acoustic test stand with

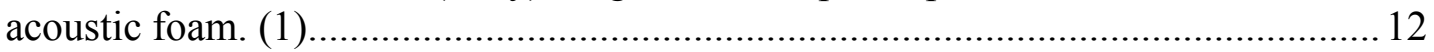

Figure 1.7: Microphone placement for torque converter near-field noise

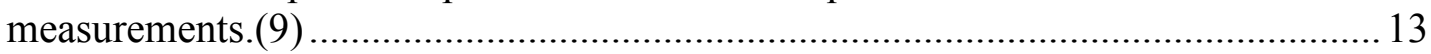

Figure 1.8: Torque converter pump with attached microwave telemetry.(11)................14

Figure 1.9: Signal evolution of the transmitter ring used for microwave telemetry

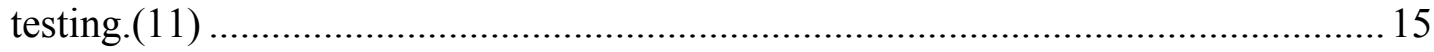

Figure 1.10: Signal evolution for the receiver modules. (11) ........................................ 16

Figure 1.11: Diagram of the mass on a conveyor belt vibration system........................ 19

Figure 1.12: Side view of an airfoil in a transverse flow causing a Von Karman Vortex

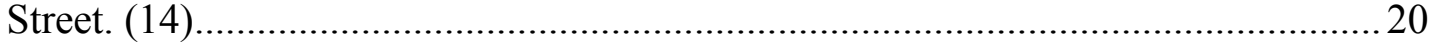

Figure 1.13: Strouhal Number for various geometries over Reynolds Number.(15).....22

Figure 1.14: Vortex-induced vibration of a spring-supported, damped circular cylinder. $\zeta$ is the damping ratio of the structure. As seen in the two data sets, the lower the damping of the structure, the more motion caused by vortex lock-in, and the longer the vortices remain locked into the motion of the cylinder. (15) 23 
Figure 1.15: Vortex suppression devices attached to cylinders; (a) helical strakes, (b) shroud, (c) axial slats, (d) streamlined fairing, (e) splitter, (f) ribboned cable, (g) pivoted guiding vane, (h) spoiler plates.(15)

Figure 1.16: Diagram of flow over a standard fan blade trailing edge, and the trailing edge of a fan made by Noctua with their Vortex-Control Notches. (16)... 25

Figure 1.17: Front and side views of a Noctua Fan with Vortex-Control Notches. (16) 25

\section{Chapter 2 - Predicting Cavitation Desinence in Automotive Torque Converters}

Figure 2.1: Cross section of an automotive torque converter; toroidal flow is indicated by the direction of the arrows with no tail. Arrows with tails indicate the normal cooling

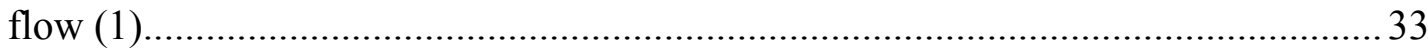

Figure 2.2: Flow incidence angle across the blades of the stator (1) ........................... 34

Figure 2.3: Left: Comparison of original torque converter dynamometer test fixture (red) and acoustic test fixture (gray). Right: Close up of opened acoustic test stand with

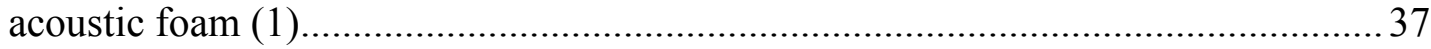

Figure 2.4: Example of torque converter speed ratio data plot .................................. 38

Figure 2.5: Visualization of the algorithm used to determine desinent cavitation in a torque converter 39

Figure 2.6: Left: Schematic of torque converter showing diameter (D) and torus length (Lt) Right: Profile of stator blade depicting maximum thickness (tmax) and chord length (lc) (1) 42

Figure 2.7: Reduction of \%RMSE and R2a during stepwise regression for round torus model. .45

Figure 2.8: RS model of desinent cavitation in constant unit input speed family..... .46

Figure 2.9: RS model of desinent cavitation in torque converters with a round torus shape 47

Figure 2.10: RS model of desinent cavitation in machined from solid torque converters48

Figure 2.11: RS model of desinent cavitation in entire torque converter population

\section{Chapter 3 - Measuring and Comparing Frequency Response Functions of Torque Converter Turbines Submerged in Transmission Fluid}

Figure 3.1: Close up image of turbine used for testing. .56 
Figure 3.2: Image of the ball bearing drop jig. The red dot is the laser vibrometer reflecting off of the trailing edge blade of the turbine. The guide tube comes down from the upper right portion of the picture to guide the ball bearing to impact near the laser dot. 58

Figure 3.3: Photo of impact rod assembly depicting the various parts used. 59

Figure 3.4: Plot comparing FRFs taken using a ball bearing as the excitation and the impact rod.

Figure 3.5: Plot comparing the autopower spectra of the "Ideal" impact with the ball bearings with the measured impact with the impact rod.

Figure 3.6: FRF plot of driving point on a trailing edge blade. 63

Figure 3.7: FRF plot of driving point on edge of shell. 64

Figure 3.8: Plot of FRFs of the dry turbine and the wet turbine. The wet turbine has had its frequency scaled in an attempt to remove the additional mass coupling caused by the fluid. 65

\section{Chapter 4-Characterizing Torque Converter Turbine Noise}

Figure 4.1: Cross section of an automotive torque converter; toroidal flow is indicated by the direction of the arrows with no tail. Arrows with tails indicate the normal cooling flow (1) 70

Figure 4.2: Nearfield acoustic measurement of automotive torque converter equipped with an un-notched trailing edge turbine (Top) and a notched trailing edge turbine (Bottom) during simulated vehicle launch on dynamometer with an input torque of $350 \mathrm{Nm}$. The area circled in white denotes the Turbine noise 73

Figure 4.3: Comparison of noise from an un-notched "Noisy" turbine and a notched quiet turbine during simulated vehicle launch with an input torque of $350 \mathrm{Nm}$. 74

Figure 4.4: Side view of an airfoil in a transverse flow causing a Von Karman Vortex Street.(3) 75

Figure 4.5: Strouhal Number for various geometries over Reynolds Number. (4).........76

Figure 4.6: Vortex-induced vibration of a spring-supported, damped circular cylinder. $\zeta$ is the damping ratio of the structure. As seen in the two data sets, the lower the damping of the structure, the more motion caused by vortex lock-in, and the longer the vortices remain locked into the motion of the cylinder. (4) 77

Figure 4.7: Diagram of flow over a standard fan blade trailing edge, and the trailing edge of a fan made by Noctua with their Vortex-Control Notches. (5). 78 
Figure 4.8: Photographs of an un-notched turbine that exhibits the "Turbine Noise" (Left) and a notched turbine (Right). 79

Figure 4.9: Comparison of autopower response of turbines known to be noisy during operation (Both un-notched turbines) to those that were known to be quiet during operation (MFS and both notched turbines) in air. 80

Figure 4.10: Comparison of frequency response functions of a reference point on the hub of the un-notched turbine of a noisy turbine with four trailing edge blades tested in air. 81

Figure 4.11: Comparison of frequency response functions of a reference point on the hub of the un-notched turbine of a noisy turbine with four trailing edge blades zoomed in to the natural frequencies thought to be associated with the trailing edge blades tested in air. 81

Figure 4.12: Comparison of frequency response functions of a reference point on the hub of the un-notched turbine of a noisy turbine with four trailing edge blades tested in ATF. 83

Figure 4.13: Left: Comparison of original torque converter dynamometer test fixture (Red) and acoustic test fixture (Gray). Right: Close up of opened acoustic test stand with acoustic foam. (1) 84

Figure 4.14: Comparison of Torque Converter turbine noise at various input torque steps. 85

Figure 4.15: Photographs of un-notched turbine trailing edge with dummy transducers (Left) and wires combined with dummy transducers (Right) ................................ 86

Figure 4.16: SPL plot comparing test alterations to turbine trailing edge. .87

Figure 4.17: Polycarbonate Extension Ring used to facilitate microwave telemetry measurements. 88

Figure 4.18: Cross-section of assembled telemetry torque converter. The polycarbonate extension ring and the microwave transmitter are annotated. 88

Figure 4.19: Spectrogram of trailing edge accelerometer measurement on automotive torque converters equipped with an un-notched (Top) and notched (Bottom) trailing edge turbine during simulated vehicle launch with an input torque of $350 \mathrm{Nm}$. 90

Figure 4.20: Spectrogram of trailing edge pressure measurement on automotive torque converter equipped with an un-notched (Top) and notched (Bottom) trailing edge turbine during simulated vehicle launch with an input torque of $350 \mathrm{Nm}$. 92

Figure 4.21: Spectrogram of trailing edge strain gage measurement on automotive torque converter equipped with an un-notched (Top) and notched (Bottom) trailing edge turbine during simulated vehicle launch with an input torque of $350 \mathrm{Nm}$. 93 
Figure 4.22: Estimated turbine exit mean velocity at each tested input torque over speed ratio.

Figure 4.23: Plots of estimated vortex frequency (Top) and filtered sound pressure level (Bottom) over speed ratio. The noise data was taken during simulated vehicle launch testing on dynamometer with an input torque of $350 \mathrm{Nm}$. The vortex frequency was calculated using the turbine exit velocity curve for $350 \mathrm{Nm}$, a trailing edge thickness of $.6 \mathrm{~mm}$, and a Strouhal Number of .28 over the entire test. .95

Figure 4.24: SPL plot comparing noise level in converters with different stator and pump combinations. Each of the tests shown was run at $300 \mathrm{Nm}$ input torque.

Figure 4.25: Color maps comparing a stock turbine noise (Top) with the same turbine after machining the trailing edge thinner (Bottom), both tests at $350 \mathrm{Nm}$ input torque 98

\section{$\underline{\text { Appendix B - Submerged Turbine Modal Analysis }}$}

Figure B.1: Comparison of autopower response of turbines known to be noisy during operation (Both unnotched turbines) to those that were known to be quiet during operation (MFS and both notched turbines) in air. 126

Figure B.2: Comparison of frequency response functions of a reference point on the hub of the unnotched turbine of a noisy turbine with four trailing edge blades tested in air. 127

Figure B.3: Comparison of frequency response functions of a reference point on the hub of the unnotched turbine of a noisy turbine with four trailing edge blades zoomed in to the natural frequencies thought to be associated with the trailing edge blades tested in air.

Figure B.4: Comparison of frequency response functions of a reference point on the hub of the unnotched turbine of a noisy turbine with four trailing edge blades tested in ATF. 129

Figure B.5: Shell breathing mode shape of turbine in air (Left column) and in ATF (Right column) shown at the opposite motion extremes (Top and bottom). $\mathrm{MAC}=72 \%$ 130

Figure B.6: Three lobed mode shape of turbine in air (Left column) and in ATF (Right column) shown at the opposite motion extremes (Top and bottom). MAC $=69 \% \ldots 130$ 


\section{List of Tables}

\section{Chapter 2 - Predicting Cavitation Desinence in Automotive Torque Converters}

Table 2.1: Dimensionless Parameters used in Speed Ratio Correlation...........................40

Table 2.2: RS Models by Torque Converter Population minus Regression Coefficients .50

Chapter 3 - Measuring and Comparing Frequency Response Functions of Torque Converter Turbines Submerged in Transmission Fluid

Table 3.1: This table is a comparison of the first six natural frequencies that appear in the FRFs of the driving point on the shell of the turbine .............................................63 


\section{Preface}

The content of this dissertation includes several full-text journal articles. A description of the contribution by the author of this dissertation to each article follows.

In all three of the papers included in this dissertation:

- Predicting Cavitation Desinence in Automotive Torque Converters

- Measuring and Comparing Frequency Response Functions of Torque Converter Turbines Submerged in Transmission Fluid

\section{- Characterizing Torque Converter Turbine Noise}

All of the experimentation, data analysis, dimensionless correlations, and background research was performed by the author of this dissertation. The coauthors of these papers supplied valued insight and experience into the phenomena discussed. 


\section{Acknowledgements}

Very few things in the world can be accomplished without the assistance of at least one other person. I have been blessed in my life to have many people there for me as an emotional support system, a helping hand in the work shop, a sounding board for my new ideas, or just a dear friend to help me relax. There is no way I can thank everyone for what they have done for me, but I hope that this is a good start...

First, I need to thank my parents, without whom I would not be here, both in a sense that I would not exist, and in that I would not have achieved so much at this point in my life without them. They let me be my own person throughout my life, with a couple pushes here and there to keep me going down the road I'm on. I love both of you guys very much.

I was not blessed with siblings, but I was given something I consider to be better, my best friends. Most count themselves lucky to have one best friend; I have five: Tyler Schwochert, Josh Fogarty, Cory Basler, Scott Gross, and Chris Rice. You guys have always been there for me. You were always honest, wise, and yet caring. I take a little piece of each of you with me wherever I go, and because of each of you I am a better man.

I thank my entire committee, but especially Jason Blough and Chuck VanKarsen, both of whom I would not be fulfilling this task without. You guys dared me to do better, and because of that I have achieved this great task. I thank Ashok Ambardar for teaching me that knowing something is not enough, that understanding is the key. I thank Chris Passerello for teaching me that although it can be arduous and time consuming, dynamics truly is the most fun you can have with a piece of chalk. All those who have been in the lab working with me also need thanks, for your support, as well as for the comic relief. Final thanks go out to VK, Keske, and Brandon, who have all been both coworkers and dear friends.

I thank all of the folks at General Motors, most especially Jean Schweitzer, for the fiscal assistance and guidance throughout this project. I know times were tough there for a while, but you stood by through it all and help make this all of the needs of this project come to fruition.

Lastly I would like to thank the many others who have contributed to this task and helped to make my journey fantastic. This includes all of the MEEM Staff, the people at IR Telemetrics, and the many other friends, family, and coworkers that I couldn't list here. Thank you all. 
xviii 


\section{Abstract}

These investigations will discuss the operational noise caused by automotive torque converters during speed ratio operation. Two specific cases of torque converter noise will be studied; cavitation, and a monotonic turbine induced noise. Cavitation occurs at or near stall, or zero turbine speed. The bubbles produced due to the extreme torques at low speed ratio operation, upon collapse, may cause a broadband noise that is unwanted by those who are occupying the vehicle as other portions of the vehicle drive train improve acoustically. Turbine induced noise, which occurs at high engine torque at around 0.5 speed ratio, is a narrow-band phenomenon that is audible to vehicle occupants currently. The solution to the turbine induced noise is known, however this study is to gain a better understanding of the mechanics behind this occurrence.

The automated torque converter dynamometer test cell was utilized in these experiments to determine the effect of torque converter design parameters on the offset of cavitation and to employ the use a microwave telemetry system to directly measure pressures and structural motion on the turbine. Nearfield acoustics were used as a detection method for all phenomena while using a standardized speed ratio sweep test. Changes in filtered sound pressure levels enabled the ability to detect cavitation desinence. This, in turn, was utilized to determine the effects of various torque converter design parameters, including diameter, torus dimensions, and pump and stator blade designs on cavitation. The on turbine pressures and motion measured with the microwave telemetry were used to understand better the effects of a notched trailing edge turbine blade on the turbine induced noise. 


\section{Chapter 1 - Literature Review}

This chapter is the review of the important literature with regards to automotive torque converters, experimental cavitation detection, and Von Karman vortex shedding.

\subsection{The Automotive Torque Converter}

Several definitions need to be made to further discuss the automotive torque converter which is a member of a much larger family of machines called turbomachines. Turbomachines are a class of devices that utilize continuously flowing fluids to transfer energy to or from one or more rows of rotating blades. A subset of turbomachines is the pump in which energy is imparted to a working fluid; this subset includes propellers, impellors, fans, and compressors. Turbines are turbomachines that receive and utilize the energy of a fluid in motion. Common turbines are found in hydroelectric power generators, flow meters, torque converters, and windmills. Flow through these machines can be axial, which is parallel to the axis of rotation, radial, which is perpendicular to the axis of rotation, or a combination of these. Lastly, turbomachines can be classified by the extent which the fluid affects the machine. Open machines have a control volume that is theoretically infinite, such as wind turbines and propellers. Closed machines are those such as pumps, water turbines, and torque converters which have a finite control volume. In general, open machines are more difficult to test and analyze without making a large number of assumptions, therefore they will not be further discussed in this paper.

The automotive torque converter is the powertrain component that multiplies engine torque, acts to augment a much smaller flywheel than what is found in manual transmission applications, and allows the engine to idle. Modern automotive torque converters are made up of three elements, a mixed flow pump and turbine, and an axial flow stator. Figure 1.1 is a basic schematic of a torque converter, including arrows showing the direction of the toroidal flow induced during operation. Maximum efficiency is achieved during high speed ratio operation while maximum torque multiplication is attained at stall, or turbine speed equal to zero. Speed ratio (SR) is defined as the ratio of the turbine speed over the pump speed. 


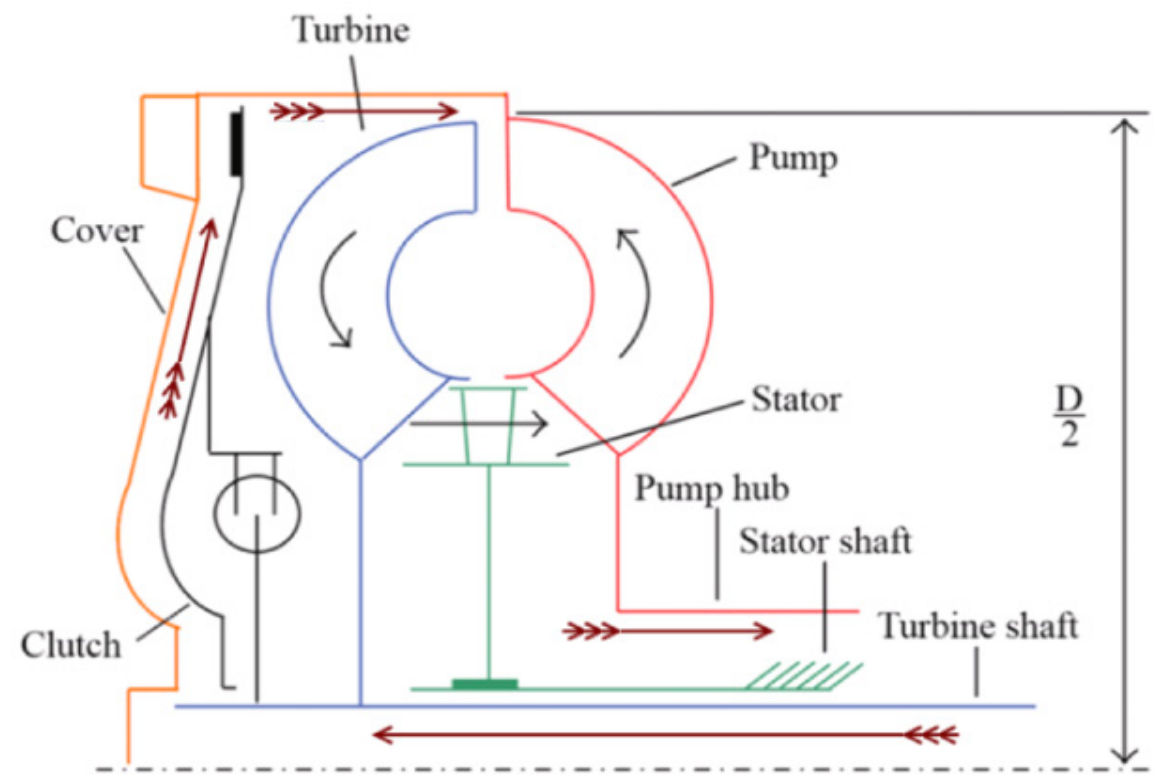

Figure 1.1: Cross section of an automotive torque converter; toroidal flow is indicated by the direction of the arrows with no tail. Arrows with tails indicate the normal cooling flow. (1)

In the basic operation of a torque converter, the pump is driven by the engine through connection of the engine crankshaft and flexplate to the cover of the torque converter. Rotation of the pump imparts angular momentum onto the automatic transmission fluid (ATF). The fluid flows from the inner radius of the pump flow path to the outer radius, and is directed into the turbine. The turbine turns the flow and directs it radially inward to the stator which guides the flow back into the pump. Fluid is redirected as it flows through each of the bladed elements shown in Figure 1.1. The change in the angular momentum of the fluid across a particular element results in a torque being applied to the shaft attached to that element. Therefore, angular momentum change of the fluid across the turbine imparts a torque on the turbine shaft which is the input shaft to the automatic transmission. The stator, which is designed to redirect the flow from the turbine into a favorable angle with regards to pump operation, creates another change in the fluid's angular momentum, creating another torque across the stator. Since the stator is fixed via a one-way clutch to a static shaft attached to the transmission housing, this torque is absorbed. It is the torque being absorbed by the stator that creates torque multiplication. Equation 1 shows the equation created via free body analysis of the three elements of the torque converter. The torque multiplication factor calculated by the torque ratio (TR) of 
the turbine over the pump is the performance characteristic that helps the vehicle accelerate from a stop.

$$
T_{\text {turbine }}=T_{\text {Pump }}+T_{\text {Stator }}
$$

Maximum torque multiplication occurs at stall. As turbine speed and speed ratio increase, torque ratio decreases. This is due in part to the change in flow incidence angle into the stator. The angle of flow exiting the turbine relative to the stator blade inlet angle decreases with increasing turbine speed such that the change in angular momentum across the stator decreases, and stator torque decreases. At a high speed ratio, the incidence angle lines up with the stator blade inlet angle, and torque multiplication diminishes to one. Up to this point of operation, the stator is grounded to the stator shaft and does not rotate. As turbine speed further increases, incidence angle rotates around the nose of the stator blade such that flow impacts the stator blades on the opposite side. To prevent a stator torque that opposes the pump from occurring, the one-way clutch on the stator assembly allows it to overrun, effectively getting out of the way of the flow. At the point when the stator overruns, the converter is acting as a fluid coupling. The speed ratio at which the stator overruns is called the coupling speed ratio. Progression of incidence angle with speed ratio is depicted in Figure 1.2.

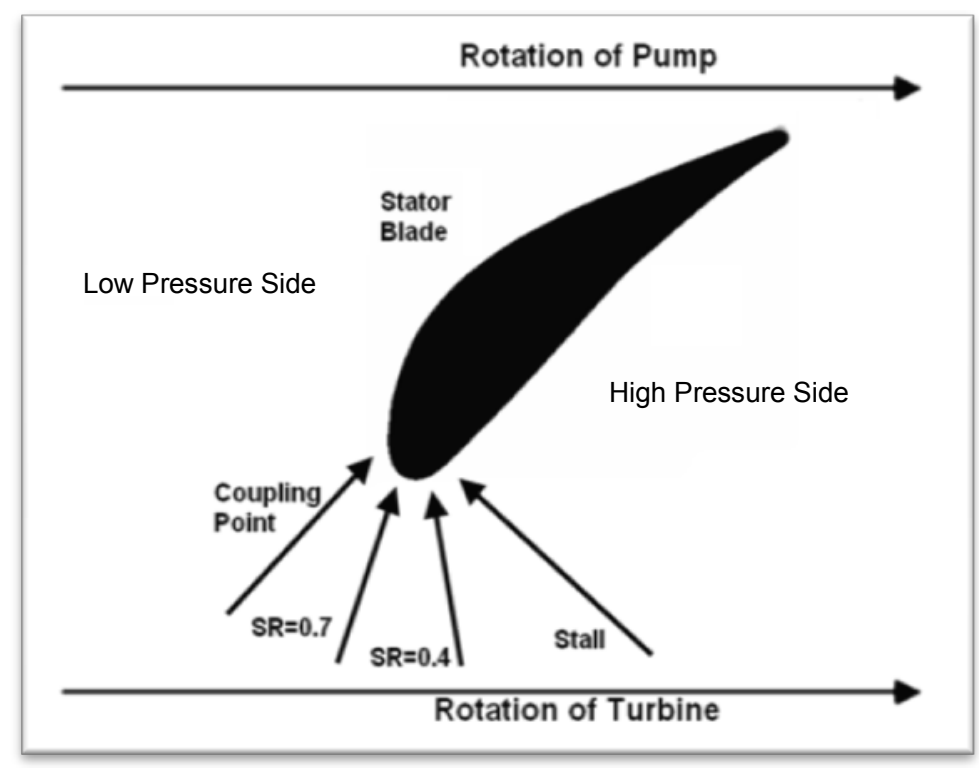

Figure 1.2: Flow incidence angle across the blades of the stator. (1) 
Torque converters need temperature control to prevent overheating of the ATF. Under practical operation, a converter functions at less than unit efficiency, and all power loss is converted to heat within the working fluid. The automatic transmission pump forces a continuous flow of cooling fluid through the torque converter. ATF flows into the converter between the cover and the clutch, and then into the outer torus region between the pump and the turbine where it is integrated into the toroidal flow. The direction of this flow is indicated in Figure 1.1 by the arrows with tails. The hotter fluid is evacuated from the converter to the vehicle cooling system. A second function of the transmission pump is to control the level of pressure in the torque converter to suppress cavitation. The flow into the converter is called charge pressure, and the flow out is called back pressure.

When the torque converter is operating at high speed ratios, a clutch can be engaged to create a direct shaft connection between the engine and transmission to bypass the hydrodynamic inefficiency of the torque converter. The clutch is engaged by reversing the cooling flow to raise the pressure behind the clutch assembly and lower the pressure between the cover and clutch. This pushes the pressure plate of the clutch toward the cover of the converter, and friction material on the clutch plate engages with the surface of the cover. Apply pressure is regulated to create either a full lock between the cover and clutch, or a controlled speed slip.

In torque converter mode, individual element torques are created from the change in angular momentum flux across an element from inlet to outlet. The torque is a function of the local static pressure times the radius integrated over the entire surface of each blade. A greater pressure differential across a blade proportionally increases the individual element torque. Consequently, at some large element torque, the pressure on the low pressure side of a blade can drop to below the vapor pressure of the fluid, and the nucleation of cavitation bubbles may occur.

The effect of cavitation on performance is dependent on whether sustained cavitation is reached. Incipient cavitation can occur at stall and dissipate before it is of any consequence. But if a high level of element torque is sustained for a long duration, the heavy cavitation will result in large vapor regions that displace the working fluid, affect 
performance, and possibly cause damage when cavitation bubbles collapse. Furthermore, collapsing bubbles cause a broadband noise that may affect the overall sound quality of the vehicle. (1) Advanced cavitation causes a decrease in individual element torques, which alters the relationship between speed and torque for the converter.

\subsection{Cavitation Detection}

Cavitation is the formation and collapse of voids within a pure liquid due to rapid high magnitude pressure fluctuation. These voids are the gaseous phase of the operating fluid. The difference between cavitating and boiling lies in the method by which the phase change occurs. Boiling is caused by changing the temperature of the liquid to the boiling temperature while the pressure is kept fairly constant, while cavitation is caused by keeping the temperature fairly constant while lowering the pressure below the vapor pressure of the fluid and thereby causing bubbles of gas to form. These are outlined better in Figure 1.3. In ideal liquids these phase diagrams accurately represent the pressure at which vapor cavities form. In the P-V diagram, following an isotherm across the liquid+vapor region is a cavitation process. The difference between boiling and cavitation is even more evident in the P-T diagram, where crossing the saturated vapor line horizontally, that is maintaining constant pressure and increasing temperature, is boiling and crossing it vertically, or dynamically decreasing pressure while somehow maintaining temperature, is cavitation. 

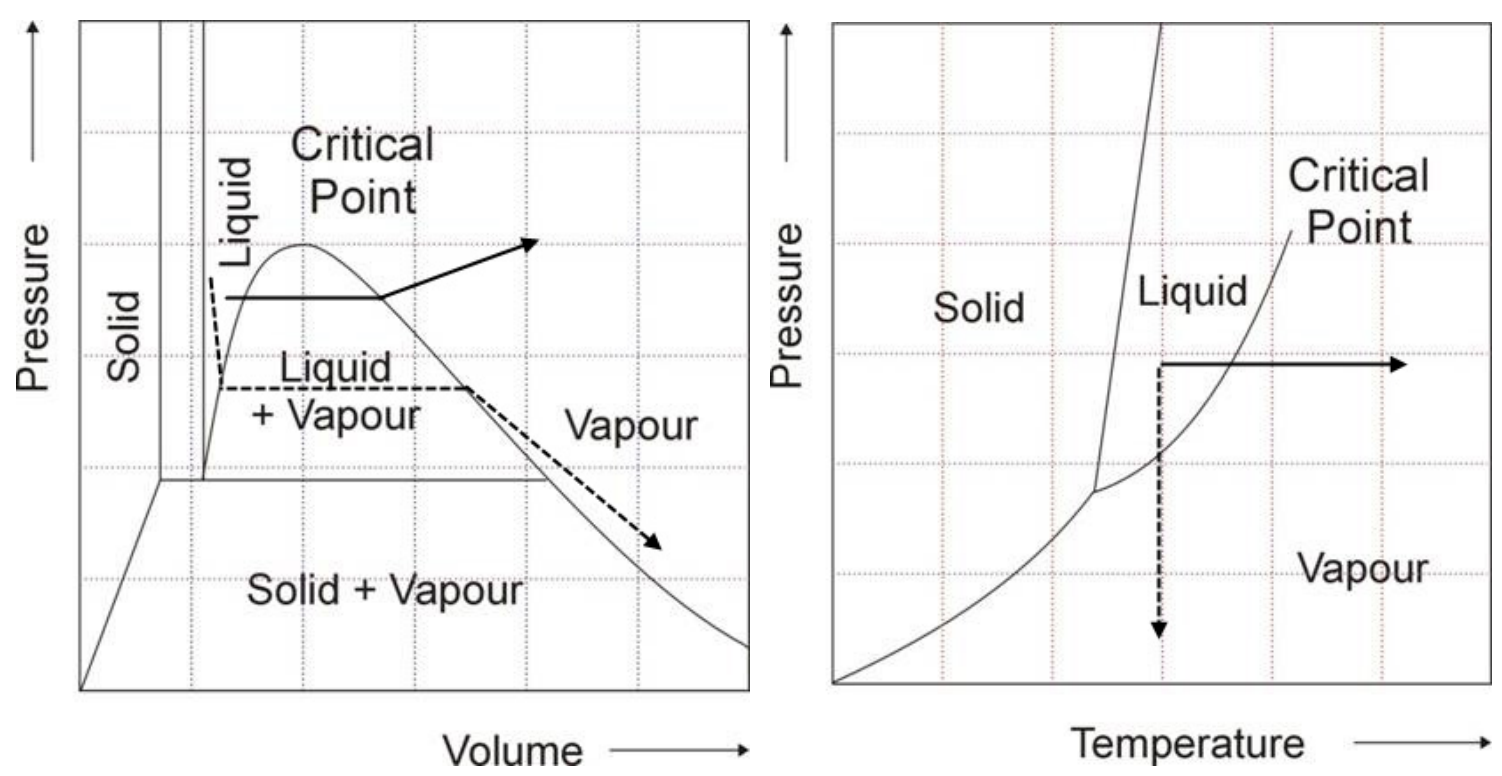

Figure 1.3: Left: Pressure - Volume phase diagram depicting difference between boiling (constant arrow) and cavitation (dashed arrow). Right: Pressure Temperature phase diagram depicting difference between boiling (constant arrow) and cavitation (dashed arrow).

Nucleation is the term used to describe the growth of cavities, or bubbles, within the fluid. (1) These nuclei are the points within the liquid where a tear or rupture may occur causing the bubble to form. Once the bubble is formed, it will either fill with the gaseous phase of the surrounding liquid or any gaseous contaminants within the liquid. The reference of these cavities as bubbles is due to the fact that they are commonly filled with the gaseous contaminants, and thus are not pure pockets of vaporized liquid. Bubble growth occurs by the dynamic reduction of pressure to the liquid vapor pressure, called vaporous cavitation. The other form of bubble formation is called gaseous cavitation that occurs when bubbles of diffused gases emerge within the fluid. This is the process which takes place when opening a can of carbonated beverage. (2)

Ultimately, liquids are not ideal substances. They contain solid, liquid, or gaseous contaminants and may have various boundary conditions that may all have an effect on the inception of cavitation bubbles.(2) Contaminants decrease the surface tension of the fluid, increasing the likelihood that a bubble can form in a specific place in the fluid. The global pressure of the fluid also has an effect on bubble formation. To characterize 
cavitation, the cavitation number, shown in Equation 2, classifies whether cavitation is occurring.

$$
\sigma_{\text {local }}=\frac{p_{\text {local }}-p_{\text {vapor }}}{\frac{1}{2} \rho V^{2}}
$$

In this equation $\mathrm{p}_{\text {local }}$ is the local pressure of the liquid, $\mathrm{p}_{\text {vapor }}$ is the vapor pressure of the bulk liquid including contaminants, $\rho$ is the density of the fluid, and $\mathrm{V}$ is the freestream velocity of the fluid. When a fluid is forced to move around solid boundaries pressure drops are caused. The nuclei that come close to those pressure drops, if they are sufficient to break the tensile strength of said liquid, will grow into a bubble and continue on to flow with the liquid portion of the fluid. These bubbles will keep growing and eventually collapsing as either the local liquid pressure increases, or the temperature drops thus increasing the tensile strength of the liquid again. Ideally, once bubbles are forming and the liquid pressure reaches the vapor pressure, Equation 2 should equal zero. Experimental results have show with pressure taps at the point of cavitation inception that liquid pressure does indeed follow this phenomenon.(2)

Experimental Cavitation Detection - Cavitation was first discovered in turbomachines as a drop in element torque which caused inefficiency in the system.(4) Since that discovery, it has been found that cavitation also causes wideband noise, material damage, and unwanted vibration. Several applications within chemical engineering have determined that the phase change induced by a cavitated flow can have ill effects on certain chemical reactions.(5)

For all of these reasons, it is vital that whenever advanced cavitation occurs that it is brought under control. Many of the previously mentioned experiments in cavitation occurred in water tunnels to simply determine the parameters that cause cavitation to occur. These were all designed to make the phenomenon easily measureable. Real life applications can be much more difficult, especially when the flow under study has already been established within a machine. Furthermore, as turbomachines can be 
difficult to model in their own right, it can be even more difficult to get measurement devices in a location to accurately measure if cavitation is occurring. Rotating blades, solid housings, high pressures, and hot fluids can all prevent an experimentalist from getting the measurements needed in order to determine the cavitation behavior of a fluid.

Fortunately, there are many specific phenomena that can easily be measured from the inception of cavitation within a flow. The collapsing of these bubbles along with the impact they make upon solid boundaries causes a broadband noise to be emitted from the liquid. Furthermore, the bubbles can be visually seen forming and collapsing within the liquid. Most invasively, pressure transducers within the flow can also be used at and along the path over which cavitation is thought to be occurring. In this last case, cavitation is detected by a wideband fluctuation of the pressure within the flow. However, placing actual transducers near or on the machine can cause a change in the cavitating flow. The rougher a surface is, the more likely the lower the liquid tensile strength and therefore any aberration on a surface can act as a nucleation point, causing an otherwise smooth surface to begin cavitation in the liquid before it normally would. Image acquisition may require a sight glass or a Plexiglas pipe be installed near the machine. This would be to great cost, and would ultimately have an effect on the flow being studied. Near-field acoustic acquisition is a completely noninvasive technique utilizing microphones near the cavitation source to detect broadband noise. Acoustic detection is the most cost efficient means of determining the presence of cavitation, and therefore will be discussed next.

Acoustic Detection - One of the signatures of a cavitating flow is the noise produced. As the local pressures within a flow vary, bubbles grow, shrink, and ultimately collapse once they have reached a location in the flow where the local pressure is greater than the tensile strength of the liquid. Furthermore, the collapse of bubbles creates more nucleation sites within the liquid. These additional nuclei are the reason why liquid history is important when attempting to analytically determine the point of cavitation. If a liquid has been previously cavitated the collapsed bubbles of a previously cavitated liquid make it more prone to cavitation additional times. The collapse of the bubbles, along with 
any impact they may make on a solid surface within the flow, all produce noise within the liquid which is ultimately radiated by the structure.

Noise generated by a cavitating flow was reported by Reynolds in 1894. (6) It was, however thought to be a curiosity until the First World War when the first attack submarines were found to be detectable due to the cavitation noise created in the propellers. As described by the early pioneers in underwater sound:

"The greater part of the underwater sound from a moving ship comes from the screw propeller. As it revolves, the pressure behind the blades is reduced and, above a certain speed, partial vacuum and unstable cavities are formed. When these "cavitations" collapse either on themselves or on the blades, noise is produced..." (7)

Cavitation noise was left forgotten, while the concerns of cavitation erosion and performance degradation were more thoroughly researched until 1940. At the dawning of the Second World War when submarines were utilized to travel covertly under newly invented RADAR technology, it was once again realized that a submarine could still be detected through hydrophones on a nearby ship. From that point on, cavitation noise research has been adopted as one of the more common detection methods.

This acoustic method is simply the use of a high sensitivity dynamic pressure sensor submerged either in the cavitating fluid far downstream from the cavitation (as is the case with hydrophones and passive SONAR on a submarine), or outside of the flow in the open air. The sound of the collapsing bubbles can be detected anywhere in the vicinity of the cavitating structure, however placing the transducer in the near-field of the structure containing the flow that is cavitating will obtain the best results. It is interesting to note that the transducer does not need to be placed directly in the vicinity of the collapsing bubbles for this detection method because the radiated sound from the bubbles collapsing is what is being measured instead of the flow along the streamline. (7) 


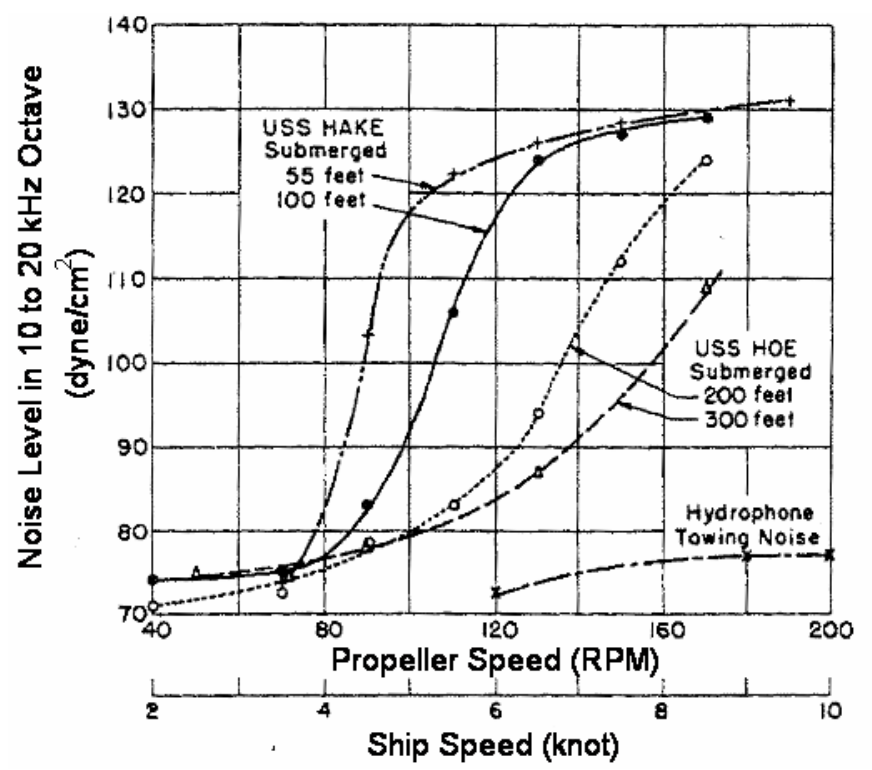

Figure 1.4: Submarine noise showing transition to noisy operation (cavitation) to depend on depth. (1)

The results of acoustic cavitation detection have a well established correlation. Figure 1.4 shows the time averaged noise level over time of a submarine in the water. As the depth of the boat increases (analogous to the controlled pressure of the fluid of operation), the speed required to increase the noise level also increases. As this noise level is a direct correlation of the number of bubbles collapsing in the flow, it also correlates with Equation 2, which is that it will take more work to create a bubble if the liquid's controlled pressure is increased. The sudden increase in the slope of the noise level dictates the inception of cavitation. At deeper depths, the rapid and sudden slope change does not occur, which indicates that either cavitation may not be occurring, the noise detected is from another point in the machine, or that the amount of cavitation occurring is very insignificant. (7) 


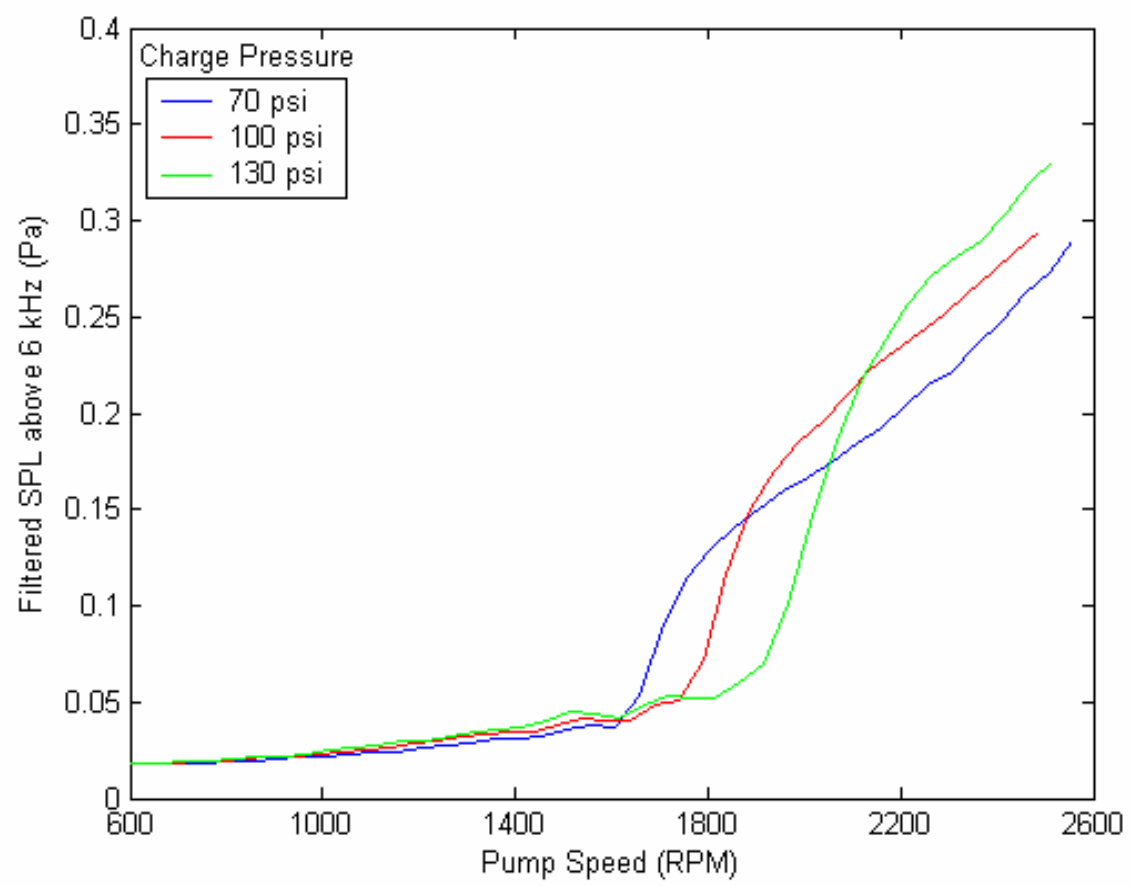

Figure 1.5: Effect of charge pressure on the onset of cavitation for a sample torque converter. (1)

Figure 1.5 depicts a filtered sound pressure signature recorded using a near-field microphone next to a torque converter as it enters cavitation. In a torque converter, cavitation is believed to occur on the airfoil shaped blades of the stator when the turbine is static or at low speeds. (1) The incidence angle of the flow across the stator blades and the speed of the flow dictate the state of cavitation. This angle of attack changes throughout torque converter operation, and is depicted in Figure 1.2.

The initial studies of cavitation have been done using a nearfield acoustic technique in a specialized torque converter test stand. This test stand and the procedures described by Kowalski in (8) and Robinette in (1) were used to determine more information regarding cavitation throughout the study detailed here. A test fixture utilizing two dynamometers to simulate automotive engine and transmission conditions was constructed for taking acoustic data with torque converters for previous projects. The fixture is much larger than typically used for torque converter testing so that it can be filled with acoustic foam to assist in absorbing and insulating noise. Figure 1.6 shows pictures of both a typical torque converter test fixture and the acoustically treated fixture used for the cavitation 
experiments. On the right is a picture of the fixture opened to reveal a torque converter and acoustic foam. A standard test procedure was developed to emulate torque converter performance during vehicle takeoff while utilizing these fixtures.

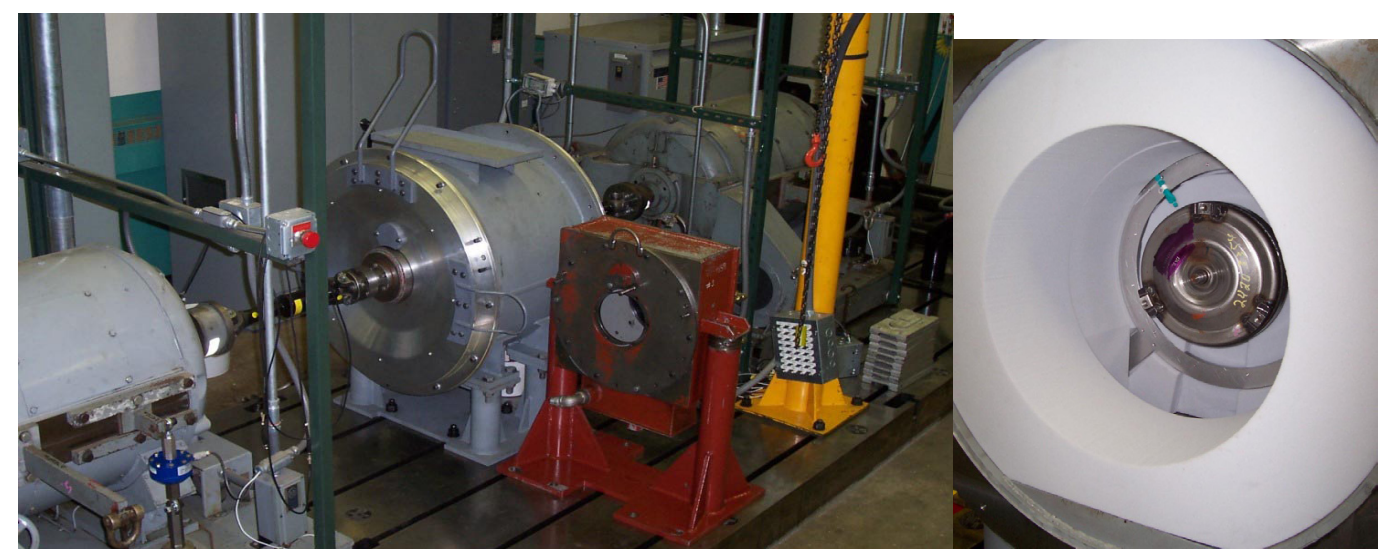

Figure 1.6: Left: Comparison of original torque converter dynamometer test fixture (Red) and acoustic test fixture (Gray). Right: Close up of opened acoustic test stand with acoustic foam. (1)

When taking acoustic data, the microphone should be as close to the machine as possible without it interfering with or coming into contact with it. The closeness to the torque converter is due to the fact that the converter is the noise of interest, and other noises that may be picked up will be much lower in magnitude with respect to the converter noise. Figure 1.7 shows a diagram of the microphone placement within the torque converter test stand used for the acoustic measurements. As seen in Figure 1.6, this particular test stand happens to be semi-anechoic as well in order to attenuate outside noise from the dynamometers and hydraulic system that operate the torque converter, as well as a majority of the reflections that could be caused by the inside of the test stand. 


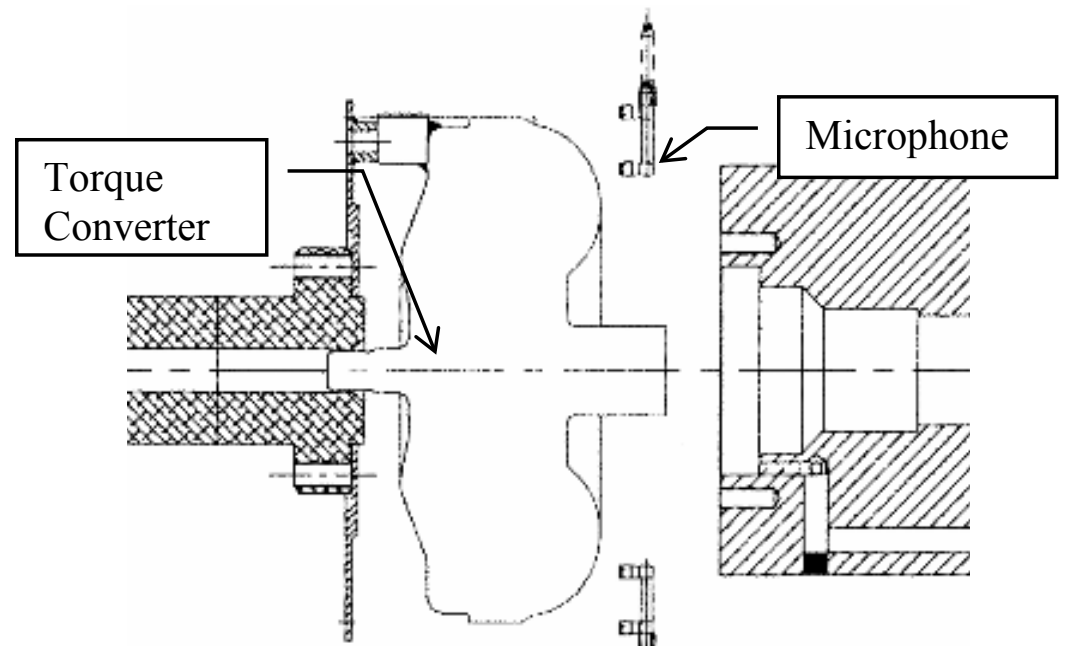

Figure 1.7: Microphone placement for torque converter near-field noise measurements.(9)

Using the noise level as an indicator function is a common means of determining the onset of cavitation; however it is not without its shortcomings. Cavitation is a broadband noise, so care needs to be taken to be sure that what is being measured is indeed cavitation and not some other broadband noise source. When making in-situ measurements or those where there are other noise sources close a high pass filter can remove a great deal of surrounding machine noise so that the high frequencies of the cavitation noise can be detected.(10)

Pressure Telemetry Measurements - As technology evolves, it becomes easier to apply transducers to complex systems and even systems that are rotating within static surfaces such as turbomachines. This wireless telemetry allows for pressure, strain, and acceleration measurements to be taken right at the tip of a rotating airfoil. A picture of a torque converter pump with attached microwave telemetry is shown in Figure 1.8. This particular pump was outfitted with pressure taps that measured the pressure fluctuations of a torque converter stator while it was cavitating.(4) 


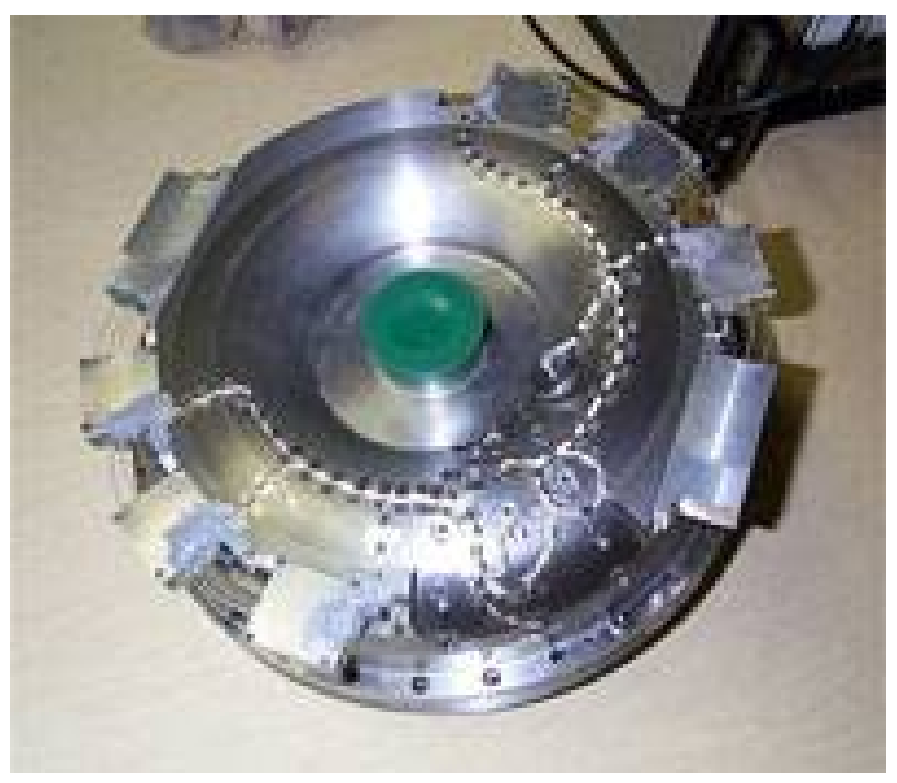

Figure 1.8: Torque converter pump with attached microwave telemetry.(11)

The principle by which the telemetry works is called a double frequency modulation (FM) technique. This is identical to radio transmission, with the exception that the electromagnetic frequencies for transmission are in the microwave realm which is the 1 to $300 \mathrm{GHz}$ range. Any transducer type may be used provided they can be adapted to interface with the telemetry system.

First the conditioned signal is converted to a one-volt amplitude square wave with a fundamental frequency in the range of frequencies of 10 and $60 \mathrm{kHz}$ corresponding to 0 and 10 volts respectively. This signal is modulated with a final $2.4 \mathrm{GHz}$ band carrier wave. Two frequencies in this band are used as signal references for high and low voltages respectively from the previous step. This final $2.4 \mathrm{GHz}$ signal is what is then transmitted. The signal evolution diagram of these steps is shown in Figure 1.9. 


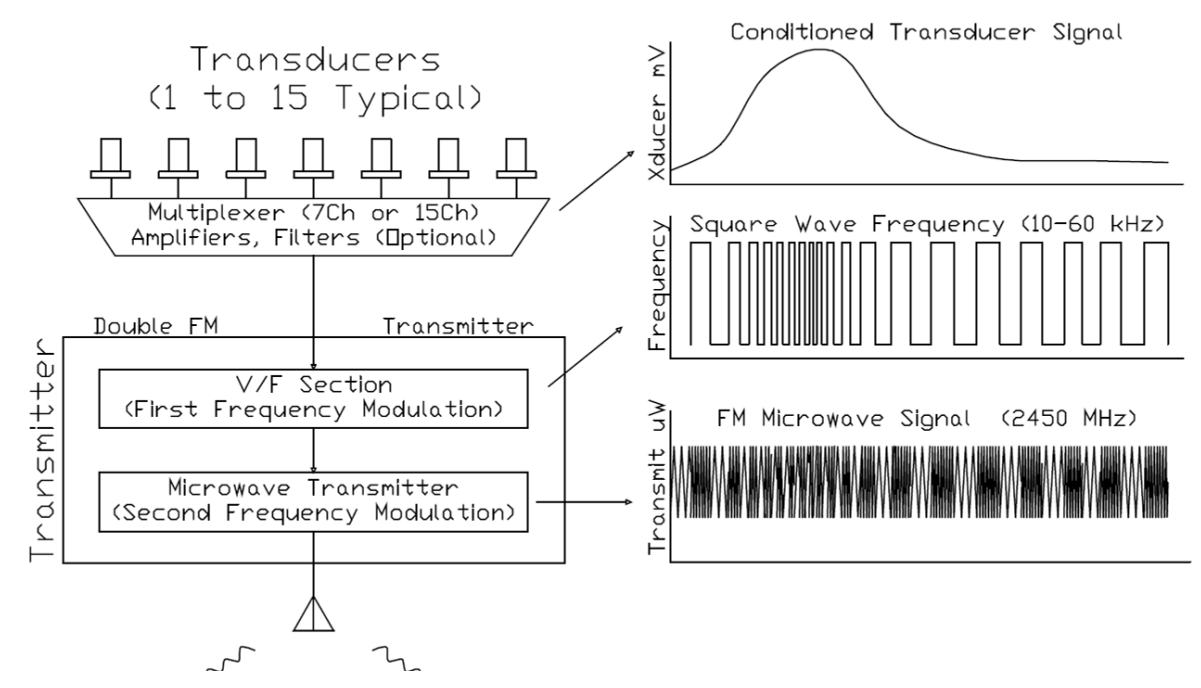

Figure 1.9: Signal evolution of the transmitter ring used for microwave telemetry testing.(11)

A microwave antenna can be set up for machines that rotate external to the flow, and the wiring can simply be machined into the part. If the part is spinning within a metal housing, a special cover made of Lexan or some other dielectric material may need to be constructed in order to allow the microwaves to pass to the receiver outside of the flow being measured.

In the case of the Zeng project (4), antennas placed inside the test stand gather the signals being transmitted and send them down a wire to an amplifier. At this point all signals from all transmitters, as well as all outside noise sources which can include car radio, wifi, and cell phone signals, are included in the signal. Once the amplifier sends the signal on to the receiver, all data from everything except the specific channel of interest are filtered out, leaving an amplitude-varied version of the signal transmitted by the corresponding transmitter.

This signal is demodulated into the square wave, which should theoretically be identical to the square wave created in the transmitter. The square wave goes through a frequency to voltage conversion (another demodulation process) and is low-pass filtered. This final signal should be nearly identical to the original conditioned signal, and is fed into the high speed data acquisition system. The signal evolution of this portion of the telemetry scheme is shown in Figure 1.10. 


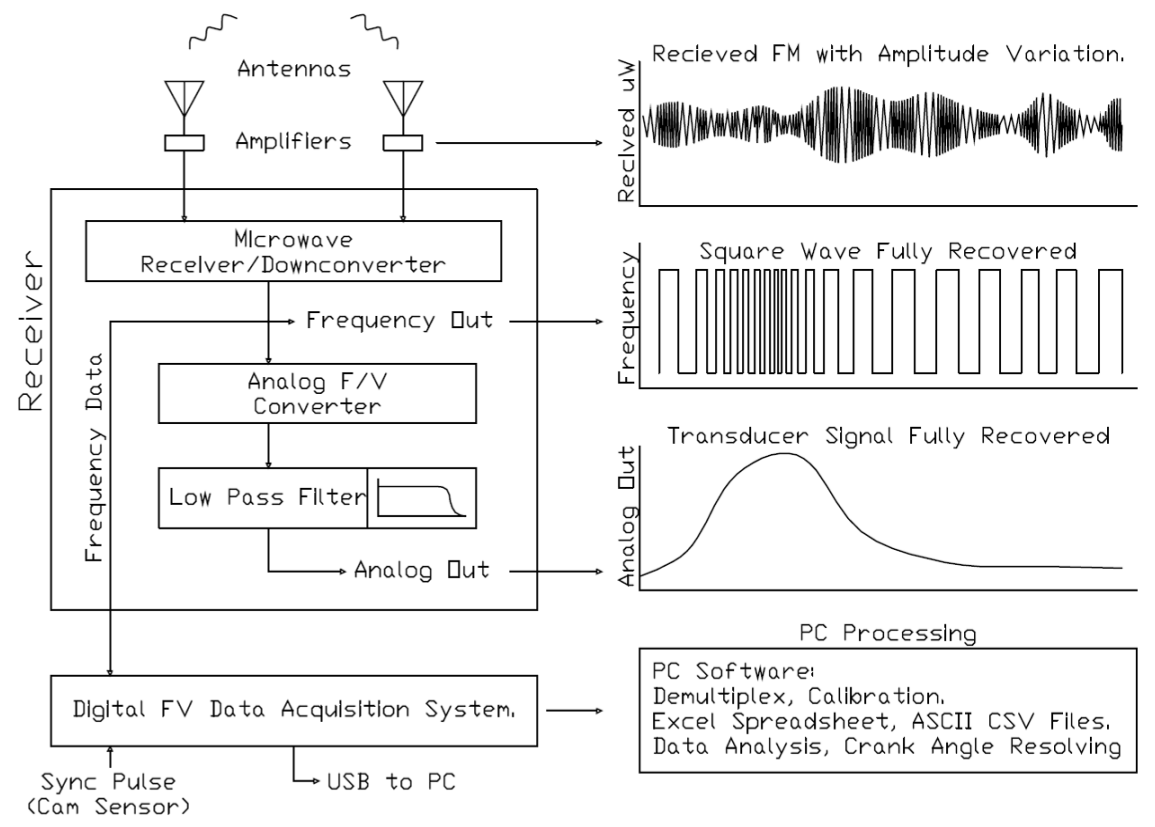

Figure 1.10: Signal evolution for the receiver modules. (11)

Another scheme that is currently being tested is an inductive transmission scheme.(11) In this method, two sets of coils are set rotating very close to each other. The primary coil attached to the telemetry is then energized with an electric current that is operating at a set carrier frequency and contains the data encoded by the telemetry inside the machine. Similar to an electric power transformer, the varying electric field creates a magnetic field which excites the second coil causing it to pick up the signal being transmitted. To enhance transmission, iron cores are used to refocus the magnetic field from around the primary coil to around the secondary.

Using telemetry coupled with fluctuating pressure measurements is, experimentally, one of the best methods that can be used to determine if cavitation is occurring at a specific place within a flow. A pressure measurement can be taken at a very specific place on the blades of a turbomachine. The pressure measurement can then be entered into Equation 2 to give an experimentally determined cavitation number. If dynamic transducers are used, the violent pressure fluctuations associated with bubble collapse can even be measured and used as an indicator of the onset of cavitation.

However, telemetry has its disadvantages. Due to the expense associated with the modifications required to be made on the rotating device, including all of the electronic 
components and balancing, this type of measurement is typically performed once. This is to learn all that can be learned about cavitation in a specific device and then it is hypothesized that geometric similitude may assist in determining how the flow is altered in similar machines. Transducer placement is very important. You cannot measure something without changing it; however you want to be as close to the phenomenon as possible in order to measure it properly. This is very true for cavitation in that adding any aberration to the surface, such as a pressure transducer or accelerometer, will potentially create a new nucleation site within the flow. Lastly, powering the electronics within the telemetry is a very important issue and is typically done by batteries, however if the inductive scheme is used, a double inductive transmission scheme can be used with one pair of coils used to power the telemetry, and another pair used to transmit the data.

Torque Converter Cavitation - In previous research, Robinette et al. in (1), (12), (9) and Kowalski et al. in (8), (13) studied torque converter cavitation in depth with the converter operating at stall. This is the condition at which a torque converter is most susceptible to cavitation due in part to the high element torques. Toroidal flow within a converter is highly three dimensional with large element flow incidence angles. The high angle of flow into the stator at stall creates regions of separated flow on the low pressure side of the blade. Research by Mekkes et al. (2) confirmed that cavitation initiates in the separated flow region at the stator inlet. As speed ratio increases, stator torque decreases, and flow incidence angle becomes less severe. Regions of separated flow are reduced, and the pressure differential across a stator blade drops, reducing the likelihood of cavitation bubbles forming at the inlet of the stator.

Mekkes (2) used wireless microwave telemetry to measure pressure at the stator inlet. The telemetry was placed on the stator, and pressure measurements were made at the inlet region of the blades. Wireless telemetry was required due to the difficulty in running wires from inside a rotating torque converter to the data acquisition equipment. The telemetry was used to acquire pressure measurements from taps on stator blades and to transmit readings to an antenna through the pump via a series of RTV filled slits milled out of the pump/stator interface. This antenna carried the signal to a receiver which 
converted it back to the raw data for a data acquisition system. A method was developed by Kowalski (8) that uses nearfield acoustic measurements to accurately detect cavitation without requiring wireless telemetry. In this way, a large population of torque converters can be tested for cavitation without the time and expense needed to create microwave instrumented torque converters for each blade design of interest. Robinette et al. (1) and (9) used the acoustic method to determine incipient cavitation at stall for a population of torque converters with a wide range of sizes and blade geometries. A correlation was formulated based on operating conditions and converter geometry to estimate the point of incipient cavitation during stall.

\subsection{Self Excited Vibration}

The study of vibratory motion has three major sub topics. Free vibration is when a system is taken out of rest for a short time, usually through some sort of impulse, and then released freely. The structure may oscillate back and forth about an equilibrium position for a short time before coming to rest. A forced vibration occurs when the input to the system happens to be some periodic function over an extended period. In most systems undergoing some form of forced vibration, the force acting on the structure is an external force, such as a motor, gears meshing, or even acoustic excitation. Self excited vibrations occur in systems where the input force on the system is a function of the motion variables of the system, that is, the displacement, velocity, or acceleration. The motion of the system couples with the system itself, in some cases causing the motion of the system to increase unbounded.

One of the basic examples of self excited vibration is the mass (m) attached to ground via a spring (with a stiffness of $\mathrm{k}$ ) that is being carried on a moving belt with a velocity of $\mathrm{v}_{0}$ by friction. The coefficient of friction $(\mu)$ of the surface between the mass and belt is usually a function of the relative velocity between them. Figure 1.11 is a diagram of this system. 


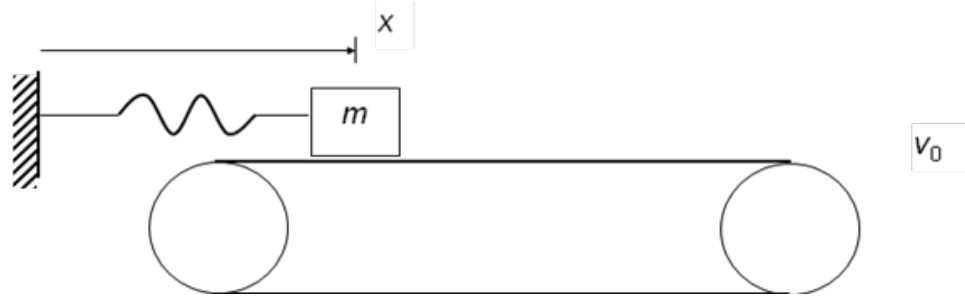

\section{Figure 1.11: Diagram of the mass on a conveyor belt vibration system.}

The equation of motion of the mass-belt system is in Equation 3. The solution to this system is far more complex than the simple spring mass system. As the spring extends due to the belt moving the mass away from ground, the force of the spring will increase until the point where it is greater than static friction. The spring force will cause the mass to slip in the opposite direction of the belt motion until the relative velocity between the belt and mass is again zero. Because kinetic friction decreases with an increase in the relative velocity of the mass and the belt, the mass slips past the original equilibrium point before "sticking" the belt due to friction taking over again. As with Figure $1.11, \mathrm{~m}$ is the mass, $\mathrm{k}$ is the stiffness of the spring, $\mu$ is the coefficient of friction, and $\mathrm{g}$ is the acceleration due to gravity. Additionally, $\mathrm{x}$ and $\ddot{x}$ are the position and the acceleration of the mass.

$$
m \ddot{x}+k x=\mu m g
$$

Equation 4 describes the entire frictional characteristic of the system. Static friction is denoted by $\mu$, and $\alpha$ is the slope parameter describing how friction changes over relative speed of the bodies. $\mathrm{v}_{0}$ is the velocity of the belt and $\dot{x}$ is the velocity of the mass.

$$
\mu=\mu_{0}\left[1-\alpha\left(\dot{x}-v_{0}\right)\right]
$$

If the velocity of the belt increases, so too does the amplitude of the motion of the mass on the belt. This result means that mathematically a negative damping term occurs when Equations 3 and 4 are combined in the final equation of motion; Equation 5. The solution to Equation 5 in the case of a negative velocity term is an exponentially growing sinusoidal response. Since the velocity of the belt and the frictional characteristic 
between the belt and the mass are sustaining the motion, and are not periodic in nature, this vibration is classified as self exciting.

$$
m \ddot{x}+\left(c-\mu_{0} \alpha m g\right) \dot{x}+k x=-\mu_{0}\left(1+\alpha v_{0}\right) m g
$$

The system shown in Figure 1.11 does not show a damper. However if damping (c) existed within the system naturally, as it does in most real dynamic systems, the magnitude of the static friction, slope parameter, and the mass could be partially offset. Therefore lightly damped systems might show a marginal instability if the mass is large enough. This concept of a negative damping term shown in Equation 5 can be used to explain phenomena such as brake squeal and clutch chatter. When frictional forces are replaced by cutting forces as in machine tools, tool chatter can be described. Lastly, when aerodynamic forces are substituted for the frictional forces in this model, aerodynamic flutter and vortex shedding can be described.

Vortex Shedding and Vortex Induced Vibration - VonKarman Vortex shedding is the unsteady flow that occurs around bluff bodies with specific fluid velocities. The flow past the object creates alternating high and low pressure vortices that form downstream of the object. Common structures on which vortices can form include power cables, bridges, offshore pipelines, heat exchangers, and towers. Figure 1.12 shows a side view of an airfoil in a transverse flow, with the alternating vortices of light and dark gray.

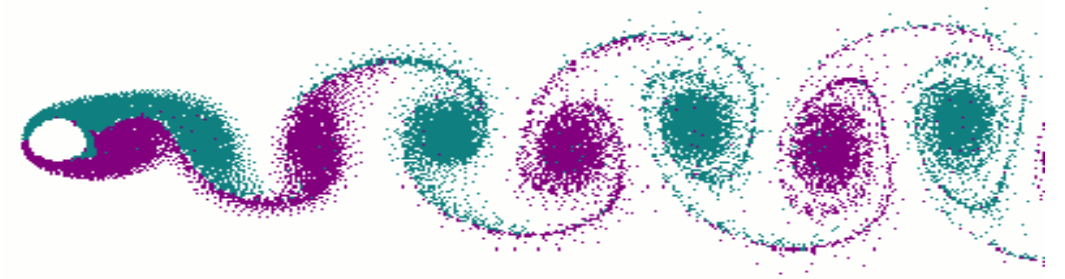

Figure 1.12: Side view of an airfoil in a transverse flow causing a Von Karman Vortex Street. (14)

Vortex shedding can occur in flows with Reynolds Numbers (Re) ranging from 47 to as high as $10^{7}$ (15) Reynolds Number, a nondimensional description of velocity, is the 
product of free stream velocity ( $U$ in meters per second) and characteristic length ( $D$ in meters), divided by kinematic viscosity ( $v$ in meters squared divided by seconds), shown in Equation 6.

$$
\operatorname{Re}=\frac{U D}{v}
$$

Often various geometries will use different characteristic lengths when calculating Reynolds Number. When used to assist in describing oscillating flows, Reynolds Number uses the characteristic lengths depicted in Figure 1.13.

The oscillating flow itself is often described using the dimensionless Strouhal Number (St). The Strouhal Number can be experimentally determined for a given geometry over a range of Reynolds Numbers. It is often observed to be relatively constant over a wide range of Reynolds Numbers for a particular geometry, and typically lies in the range of .1 to .3 for oscillating flow. The equation for Strouhal Number is shown in Equation 7.

$$
S t=\frac{f D}{V}
$$

The parameters for Strouhal Number are the vortex frequency ( $\mathrm{f}$ in $\mathrm{Hz}$ ), the characteristic length of the structure $(\mathrm{D}$, in the case of a cylinder, the diameter in meters), and the free stream velocity of the flow around the structure ( $\mathrm{U}$ in meters per second, the same as $U$ for the Reynolds Number). The range of Strouhal Number over Reynolds Number for various geometries is shown in Figure 1.13. 


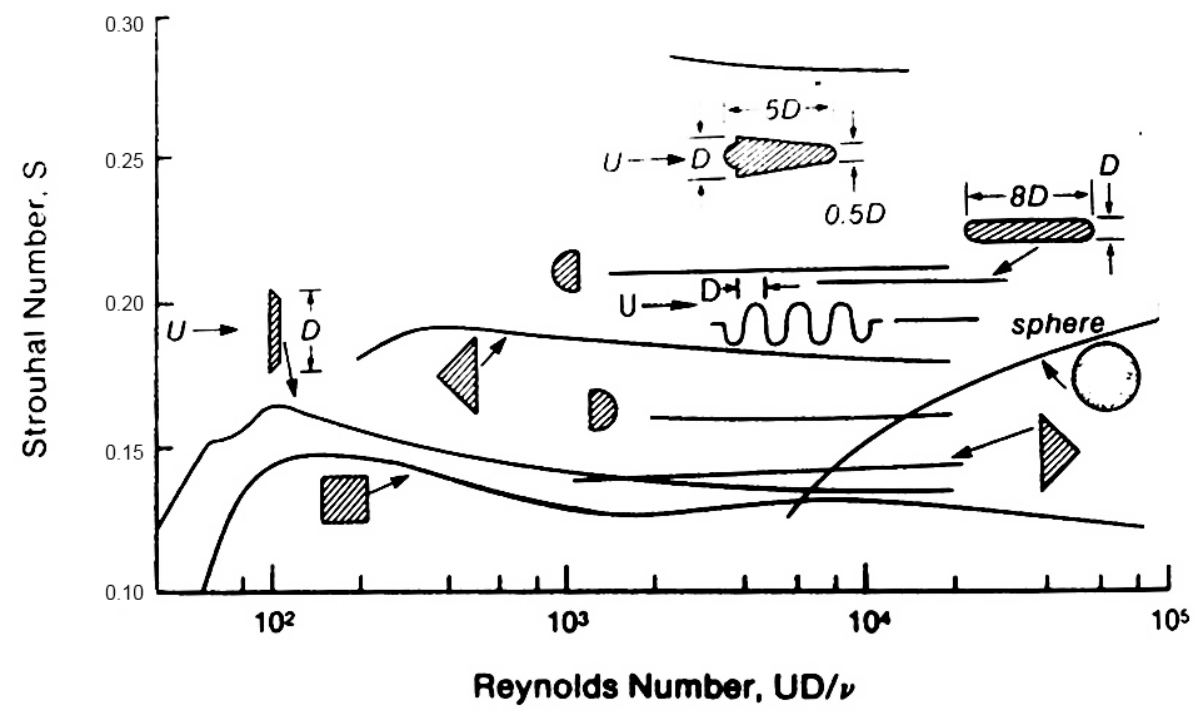

Figure 1.13: Strouhal Number for various geometries over Reynolds Number.(15)

Ultimately, the Strouhal Number is a means of determining frequency of vortex release. As each vortex sheds from the structure, the body will tend to move toward the low pressure zone left in the wake of the vortex. Therefore, if the vortex frequency matches close to a natural frequency of the structure, the structure can resonate and a self-excited vibration can occur. This is called lock-in, and while the structure resonates at its natural frequency the vortices shed at the natural frequency of the structure. The top portion of Figure 1.14 shows a plot that tracks vortex frequency as it approaches the natural frequency of the cylinder. Lock-in is the region in Figure 1.14 where the data points indicating the vortex frequency begin to follow a horizontal line at a natural frequency of the cylinder. 


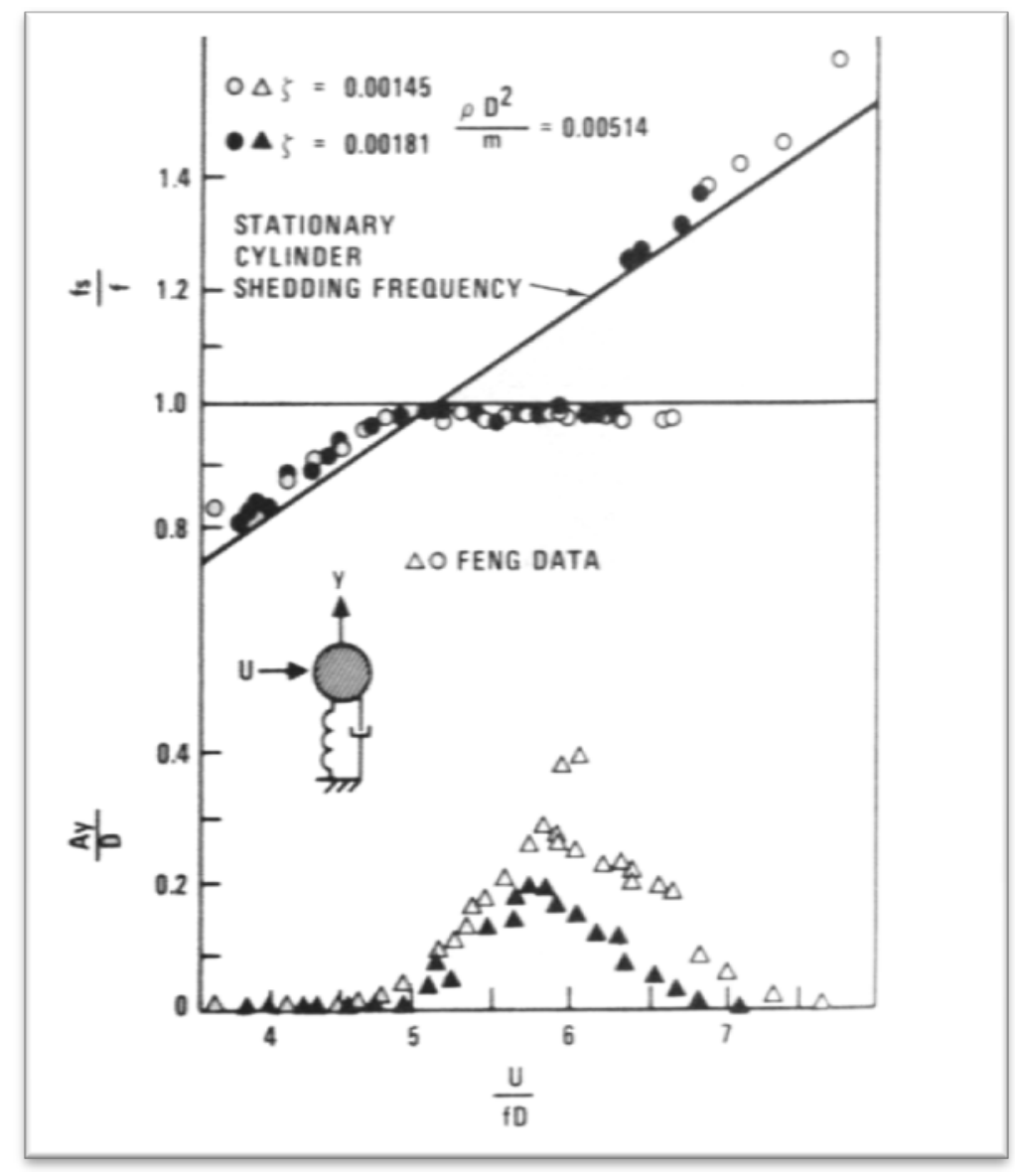

Figure 1.14: Vortex-induced vibration of a spring-supported, damped circular cylinder. $\zeta$ is the damping ratio of the structure. As seen in the two data sets, the lower the damping of the structure, the more motion caused by vortex lock-in, and the longer the vortices remain locked into the motion of the cylinder. (15)

Once the vortex frequency is theoretically far enough away from the structure's natural frequency, the vortex frequency will part from the natural frequency as the interaction between the structure and fluid becomes too weak to continue to support the feedback required for the self-excited vibration. If no natural frequency is present, the vortex frequency will remain near the "Stationary Cylinder Shedding Frequency" line indicated in Figure 1.14. The motion of the cylinder (shown in the bottom portion of Figure 1.14) is driving the vortex frequency (shown in the top portion of the figure) and vice versa. The top vertical axis of Figure 1.14 is the dimensionless vortex frequency, with $f$, the natural frequency of the system, normalizing $\mathrm{f}_{\mathrm{s}}$, the vortex frequency. The lower plot shows the amplitude of the motion of the cylinder, Ay, as it is normalized by the diameter of the 
cylinder, D. The horizontal axis in both cases is the free stream velocity of the flow, $\mathrm{U}$, as it increases. $\mathrm{U}$ is normalized by the product of the natural frequency and the diameter of the structure.

The most common means to reduce the occurrence of vortex-induced vibration is to add some vortex suppression device, such as helical strakes, axial slats, streamlined fairings, or spoiler plates. These vortex suppression devices are shown in Figure 1.15. These devices are used to suppress vortices on many structures, including high tension cables and oil rigs.

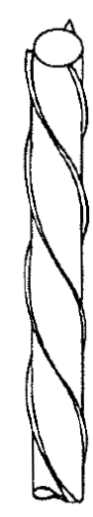

(a)

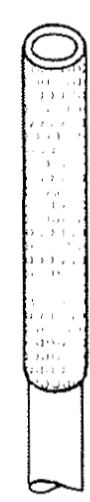

(b)

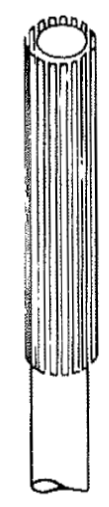

(c)

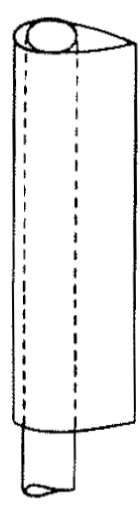

(d)

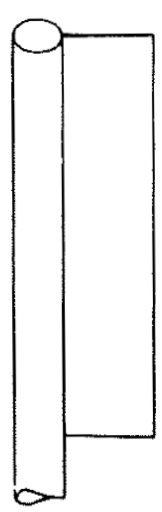

(e)

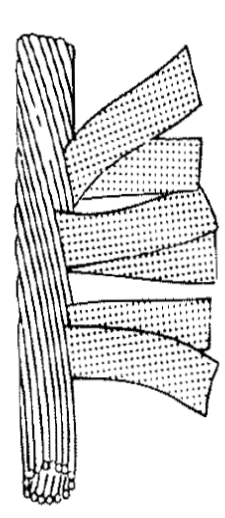

$(f)$

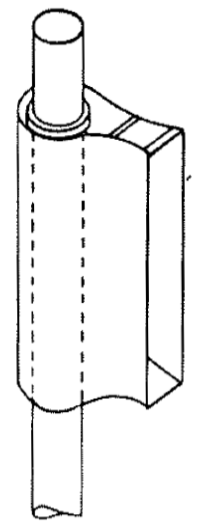

(g)

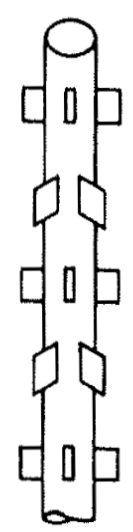

(h)

Figure 1.15: Vortex suppression devices attached to cylinders; (a) helical strakes, (b) shroud, (c) axial slats, (d) streamlined fairing, (e) splitter, (f) ribboned cable, (g) pivoted guiding vane, (h) spoiler plates.(15)

Additionally, Blevins (15) states that vortex-induced vibrations of a plate were suppressed by streamlining the trailing edge. Noctua, a fan manufacturer, advertises the use of vortex control notches which are said to reduce the noise of their fans. Figure 1.16 shows a diagram of the flow over a conventional fan trailing edge, and a trailing edge with "vortex-control" notches. (16) Noctua states that these notches create a disturbance upstream of where the vortices form, thereby breaking up the vortex street before it fully forms or preventing the vortices from coupling with the structure to produce a selfexcited vibration. 

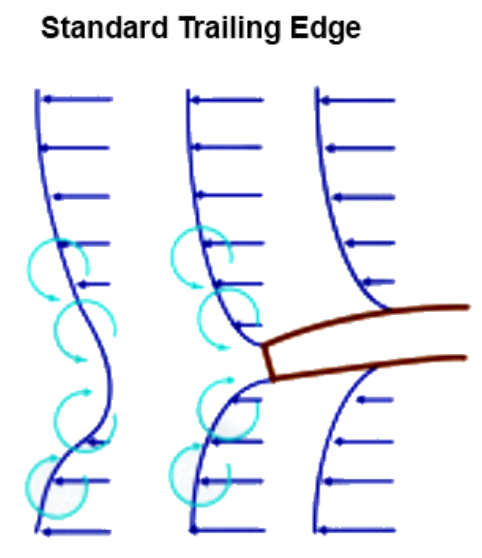

Trailing Edge with Vortex-Control Notches

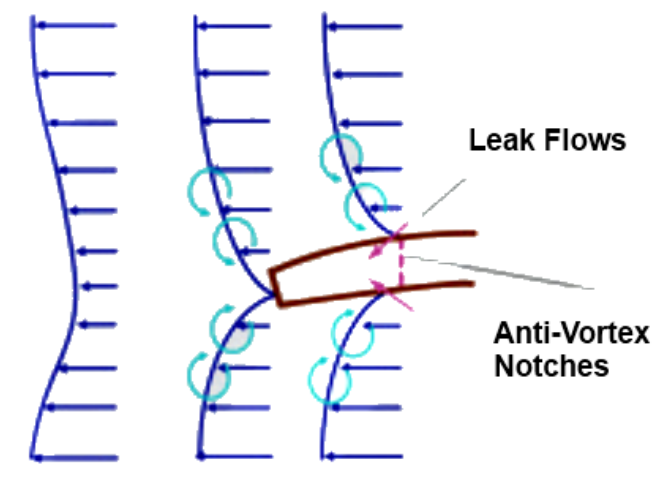

- Fan Blade - Vortices - Airflow

Figure 1.16: Diagram of flow over a standard fan blade trailing edge, and the trailing edge of a fan made by Noctua with their Vortex-Control Notches. (16)

Figure 1.17 is a front and side view of the Noctua fan with Vortex-Control Notches. The noise created by the vortices is increased due to the nine blade design of the fan. When this design was implemented it increased the flow velocity to the point where vortex noise and its interactions with the structure around the fan were more noticeable than the noise from a conventional fan. Noctua states that the two notches at the trailing edge allow the flow from the pressure and suction sides of the blade to mix smoothly which reduces the vortex noise.(16)
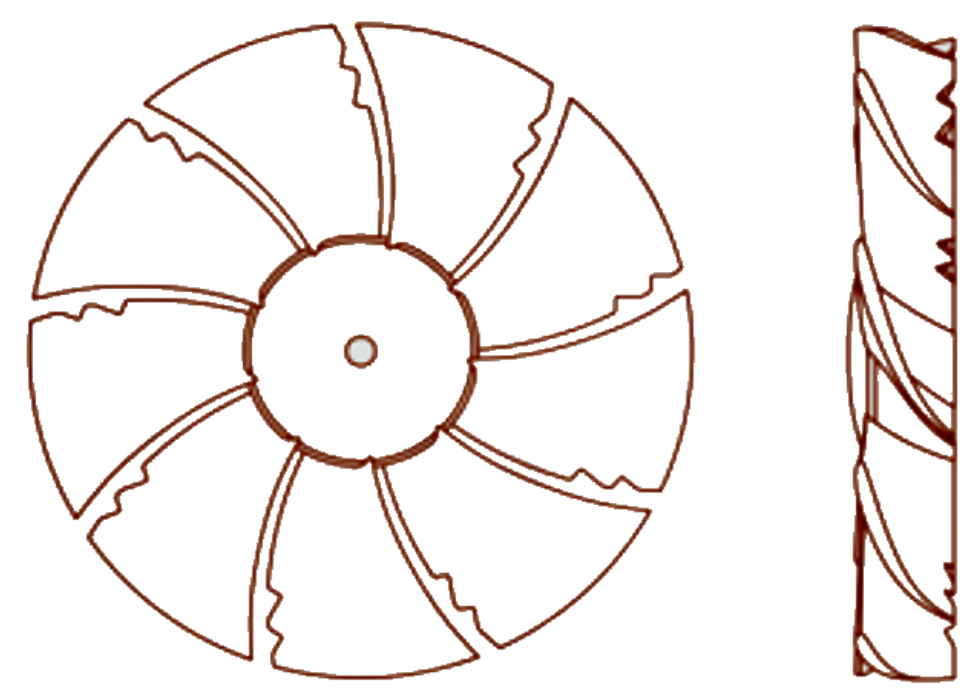

Figure 1.17: Front and side views of a Noctua Fan with Vortex-Control Notches. (16) 


\subsection{Torque Converter Turbine Noise and Cavitation Noise over Varying Speed Ratio}

The remainder of this dissertation discusses experimentation done with torque converters with respect to operation over simulated vehicle take-off. Chapter 2 is a paper discussing torque converter cavitation desinence during operation. Previous works by Robinette in (1), (12), and (9) detail torque converter cavitation during stall operation. However, a torque converter remains in stall only so long as there is not sufficient torque to create vehicle motion. If cavitation is present at stall, it will cease at some higher speed ratio as stator torque and turbine torque decrease. If cavitation at stall is at a low level, and cavitation desinence occurs shortly into the vehicle launch, then noise from cavitation will not be noticed, and its effect on performance will be of very little consequence. If, however, torque levels are high enough to sustain cavitation to a high vehicle speed, then cavitation noise could be objectionable to the vehicle operator and torque converter performance might be impacted

Chapter 3 describes the methodology used to compare the turbine dynamics in air to the dynamics in its working fluid, automatic transmission fluid. Since the structure of the turbine is more highly damped and has more virtual mass when submerged in ATF than air, the dynamic parameters of the structure might also be different. It was hypothesized that this might in some way make it possible to determine if a turbine exhibited the "Turbine Noise" phenomenon discussed in Chapter 4.

The project outlined in the Chapter 4 utilized the previously discussed wireless telemetry and acoustic methods applied to speed ratio testing to identify and detect a noisy turbine phenomenon. A 4-6 kHz narrow band noise occurred at mid to high speed ratios in some converters tested. This noise was eliminated by the addition of trailing edge notches to the turbine, similar to those described in the Figure 1.17. The "Turbine Noise" phenomenon was thought to be a result of VonKarman Vortex Shedding coupled with a natural frequency of the torque converter turbine. The experimentation discussed was performed to determine if the vortex shedding hypothesis was valid. 


\subsection{References}

(1) Robinette DL. Detecting and Predicting the onset of Cavitation in Automotive Torque Converters. PhD Dissertation, Michigan Technological University. Ann Arbor: ProQuest/UMI. 2007.

(2) Arakeri V. Cavitation Inception. Proc. Indian Acad. Sci. 1979; Vol. C2, Pt. 2: pp. 149-177.

(3) Mekkes J, Anderson CL, Narain A. Static Pressure Measurements on the Nose of a Torque Converter Stator during Cavitation. ISROMAC. 2004.

(4) Zeng L, Anderson CL, Sweger PO, Narain A. Experimental Investigation of Cavitation Signatures in an Automotive Torque Converter Using a Microwave Telemetry Technique. ISORMAC. 2002.

(5) Blander M, Katz JL. Bubble Nucleation in Liquids. American Institute of Chemical Engineers. 1975; Vol. 21, No. 5: pp. 833-847.

(6) Reynolds O. On The Dynamical Theory of Incompressible Viscous Fluids and the Determination of the Criterion. Proceedings of the Royal Society of London. 1895.

(7) Strasberg M, Taylor D. Propeller Cavitation Noise after 35 Years of Study. Noise and Fluids Engineering. 1977.

(8) Kowalski D, Anderson CL, Blough JR. Cavitation Detection in Automotive Torque Converters Using Nearfield Acoustical Measurements. SAE-NVH. 5/16/2005; 2005-01-2516.

(9) Robinette DL, Anderson CL, Blough JR, Johnson MA, Maddock DG, Schweitzer JM. Characterizing the Effect of Automotive Torque Converter Design Parameters on the Onset of Cavitation at Stall. SAE-NVH. 5/15/2007; 2007-01-2231.

(10) Alfayez L, Mba D, Dyson G. The Application of Acoustic Emission for Detecting Incipient Cavitation and Best Efficiency Point of a $60 \mathrm{Kw}$ Centrifugal Pump: Case Study. NDT \& E International. 2005; Vol. 38, Issue 5: pp. 354-358.

(11) Barna G. IR Telemetrics. 2010.

(12) Robinette DL, Schweitzer JM, Maddock DG, Anderson CL, Blough JR, Johnson MA. Predicting the Onset of Cavitation in Automotive Torque Converters-Part II: A Generalized Model. ISORMAC. 2008; Article ID 312753.

(13) Kowalski D, Anderson CL, Blough JR. Cavitation Prediction in Automotive Torque Converters. SAE-NVH. 2005; 2005-01-2557.

(14) La Rosa Siqueira C. Wikipedia. File:Vortext-street-animation.gif. 2005. http://en.wikipedia.org/wiki/File:Vortex-street-animation.gif.

(15) Blevins R. Flow Induced Vibration. Krieger Publishing Company. 2001 
(16) Noctua Fan Company. Nine Blade Design with Vortex-Control Notches. 2012; http://www.noctua.at/main.php?show=nine_blade_design\&lng=en.

(17) Raabe J. Cavitation Effects in Turbomachinery - European Experiences: Cavitation State of Knowledge. ASME Fluid Engineering Div. 6/1969.

(18) Sweger P, Anderson CL, Blough JR. Measurements of Strain on $310 \mathrm{Mm}$ Converter Turbine Blade. International Journal of Rotating Machinery. 2004; Volume 10, No 1: pp. 55-63.

(19) Wen Y, Henry M. Time Frequency Characteristics of the Vibroacoustic Signal of Hydrodynamic Cavitation. ASME Journal of Vibration and Acoustics. 2002; Vol 124: pp. 469-476.

(20) Arakeri VH, Acosta AJ. Viscous Effects in the Inception of Cavitation on Axisymmetric Bodies. ASME Journal of Fluids Engineering. 12/1973; pp. 519527.

(21) Baldassarre A, DeLucia M, Nesi P. Real-Time Detection of Cavitation for Hydraulic Turbomachines. Real-Time Imaging. 1998; pp. 403-416.

(22) Blake WK, Wolpert MJ, Geib FE. Cavitation Noise As Influenced By Boundary Layer Development on a Hydrofoil. Journal of Fluid Mechanics. 1977; Vol. 80: pp. 617-640.

(23) Brennen CE. Cavitation and Bubble Dynamics. Oxford University Press. 1995; .

(24) Caron JF, Farhat M, Avellan F. Physical Investigation of the Cavitation Phenomenon. 4th International Symposium on Cavitation. 6/2001.

(25) Chahine GL. Nuclei Effects on Cavitation Inception and Noise. 25th Symposium on Naval Hydrodynamics. 8/2004.

(26) Chen Y, Heister SD. Two-Phase Modeling of Cavitated Flows. Computers and Fluids. 1995; Vol. 24, No. 7: pp. 799-809.

(27) $\mathrm{Gu} \mathrm{W}, \mathrm{He} \mathrm{Y}, \mathrm{Hu}$ T. Transcritical Patterns of Cavitating Flow and Trends of Acoustic Level. ASME. 2001; Vol. 123: pp. 850-857.

(28) Guennoun F, Farhat M, Bouziad YA, Avellan F. Experimental Investigation of a Particular Traveling Bubble Cavitation. 5th International Symposium on Cavitation. 2003.

(29) Knapp RT, Hammitt JW, Daily FG. Cavitation. Engineering Societies Monographs. 1970.

(30) Kunz R, Boger D, Stinebring D, Checzewski T. A Preconditioned Navier-Stokes Method for Two-Phase Flows with Application to Cavitation Prediction. Computers and Fluids. 2000; Vol. 29: pp. 849-875. 
(31) Martin CS, Medlarz H, Wiggert DC, Brennen C. Cavitation Inception in Spool Valves. Transaction of ASME. 1981; Vol. 103: pp. 564-576.

(32) McNulty PJ, Pearsall IS. Cavitation Inception in Pumps. ASME Journal of Fluids Engineering. 1982; Vol. 104: pp. 99-104.

(33) Robinette DL, Schweitzer JM, Maddock DG, Anderson CL, Blough JR, Johnson MA. Predicting the Onset of Cavitation in Automotive Torque Converters - Part II: A Generalized Model. International Journal of Rotating Machinery. 2008; Article ID 312753. 


\title{
Chapter 2 - Predicting Cavitation Desinence in Automotive Torque Converters ${ }^{1}$
}

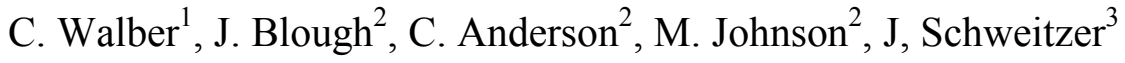 \\ ${ }^{1}$ PCB Piezotronics \\ 3425 Walden Ave Depew, New York, USA \\ email: cmwalber@mtu.edu \\ ${ }^{2}$ Michigan Technological University, Department of Mechanical Engineering \\ 1400 Townsend Dr. Houghton, Michigan, USA \\ ${ }^{3}$ General Motors Powertrain \\ Pontiac, Michigan, USA
}

\subsection{Abstract}

Cavitation is a concern in automotive torque converters because it can cause operation to be altered from design intent, generate unaccepTable 2.noise in the passenger compartment of a vehicle, and damage torque converter components in extreme situations. Previous cavitation experiments have determined the onset of cavitation in stall mode. During actual operating conditions, however, torque converter stall occurs for a short duration. Thus characterization of cavitation in speed ratio operation is needed. Torque converter speed, torque, and hydraulic parameters at cavitation desinence were determined experimentally using a dynamometer test cell in concert with nearfield acoustic measurements. These experimentally determined operating conditions along with design parameters were used to create a set of dimensionless parameters, which were used to develop non-dimensional predictive models of the cessation of cavitation during simulated vehicle takeoff. Models were developed for several groups of torque converters with varying degrees of similitude.

${ }^{1}$ The material contained in this chapter has been submitted to the International Journal of Rotating Machinery. 


\subsection{Introduction}

The automotive torque converter is the powertrain component that multiplies engine torque to overcome the poor low speed torque characteristics of an internal combustion engine. It regulates engine speed to evenly increase during vehicle acceleration. Modern automotive torque converters are made up of three elements, a mixed flow pump and turbine, and an axial flow stator. Figure 2.1 is a basic schematic of a torque converter, including arrows showing the direction of the toroidal flow induced during operation. Maximum efficiency is achieved during high speed ratio operation while maximum torque multiplication is attained at stall, or turbine speed equal to zero. Speed ratio (SR) is defined as the ratio of the turbine speed over the pump speed.

In the basic operation of a torque converter, the pump is driven by the engine through connection of the engine crankshaft and flexplate to the cover of the torque converter. Rotation of the pump imparts angular momentum onto the automatic transmission fluid (ATF). The fluid flows from the inner radius of the pump flow path to the outer radius, and is directed into the turbine. The angular momentum of the fluid acts on the turbine, imparting a torque to the turbine shaft which is the input shaft to the automatic transmission. The fluid exits the turbine and is redirected by the stator toward the pump at a favorable angle with regards to pump operation. The change in angular momentum by the stator is what creates torque multiplication. The torque multiplication factor, called torque ratio (TR), is the performance characteristic that helps the vehicle accelerate from a stop. 


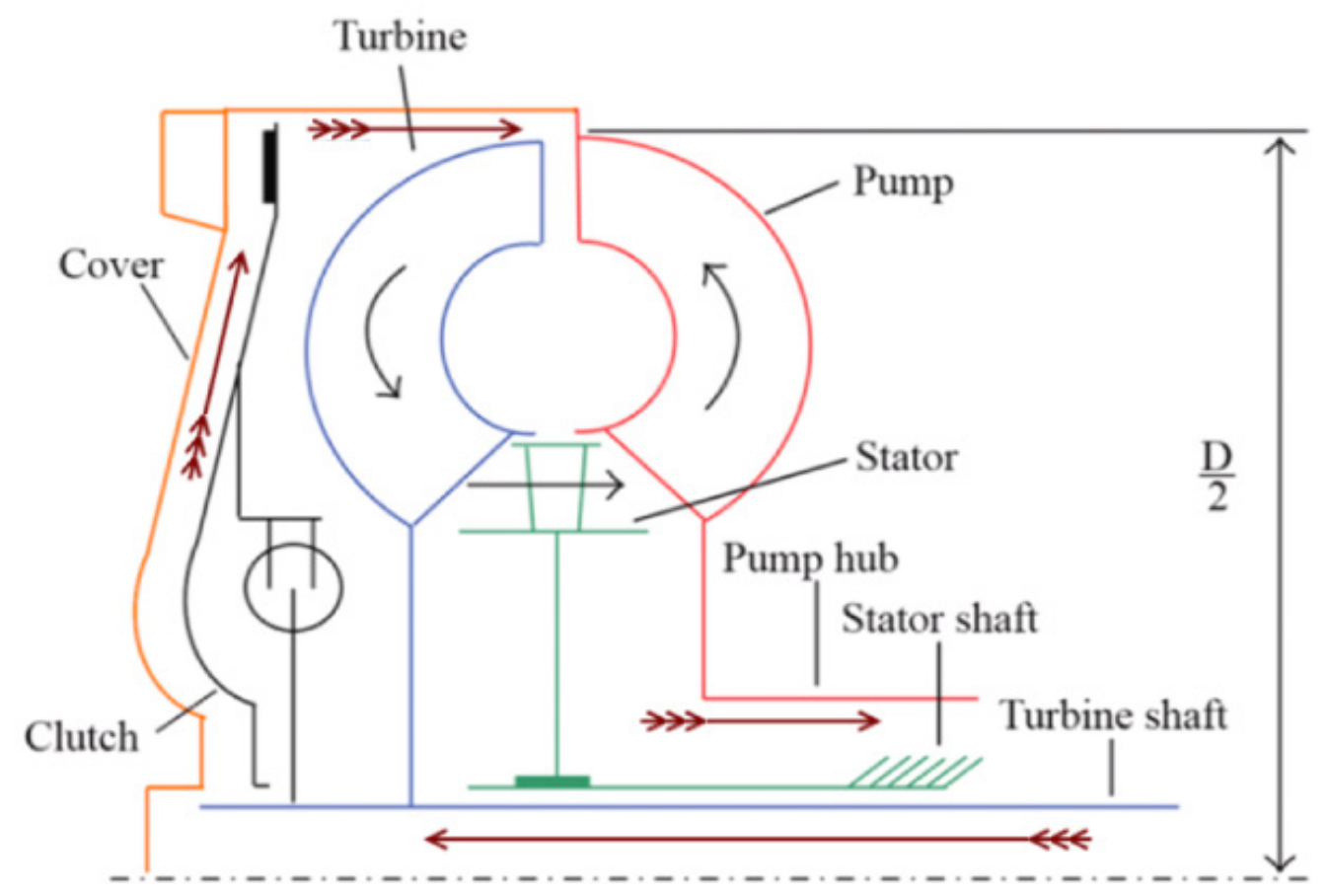

Figure 2.1: Cross section of an automotive torque converter; toroidal flow is indicated by the direction of the arrows with no tail. Arrows with tails indicate the normal cooling flow (1)

Maximum torque multiplication occurs at stall. As turbine speed and speed ratio increase, torque ratio decreases. This is due in part to the change in flow incidence angle into the stator. The angle of flow exiting the turbine relative to the stator blade inlet angle decreases with increasing turbine speed such that the change in angular momentum across the stator decreases, and stator torque decreases. At a high speed ratio, the incidence angle lines up with the stator blade inlet angle, and torque multiplication becomes one. Up to this point of operation, the stator is grounded to the stator shaft and does not rotate. As turbine speed further increases, incidence angle rotates around the nose of the stator blade such that flow impacts the stator blades on the opposite side. To prevent torque ratio from becoming less than one, the stator assembly has a one-way clutch that allows it to overrun, effectively getting out of the way of the flow. At the point when the stator overruns, the converter is acting as a fluid coupling. The speed ratio at which the stator overruns is called the coupling speed ratio. Progression of incidence angle with speed ratio is depicted in Figure 2.2. 
Torque converters need temperature control to prevent overheating of the ATF. Under practical operation, a converter functions at less than unit efficiency, and all power loss is converted to heat within the working fluid. The automatic transmission pump forces a continuous flow of cooling fluid through the torque converter. ATF flows into the converter between the cover and the clutch, and then into the outer torus region between the pump and the turbine where it is integrated into the toroidal flow. The direction of this flow is indicated in Figure 2.1 by the maroon arrows. The hotter fluid is evacuated from the converter to the vehicle cooling system. A second function of the transmission pump is to control the level of pressure in the torque converter to suppress cavitation. The flow into the converter is called charge pressure, and the flow out is called back pressure.

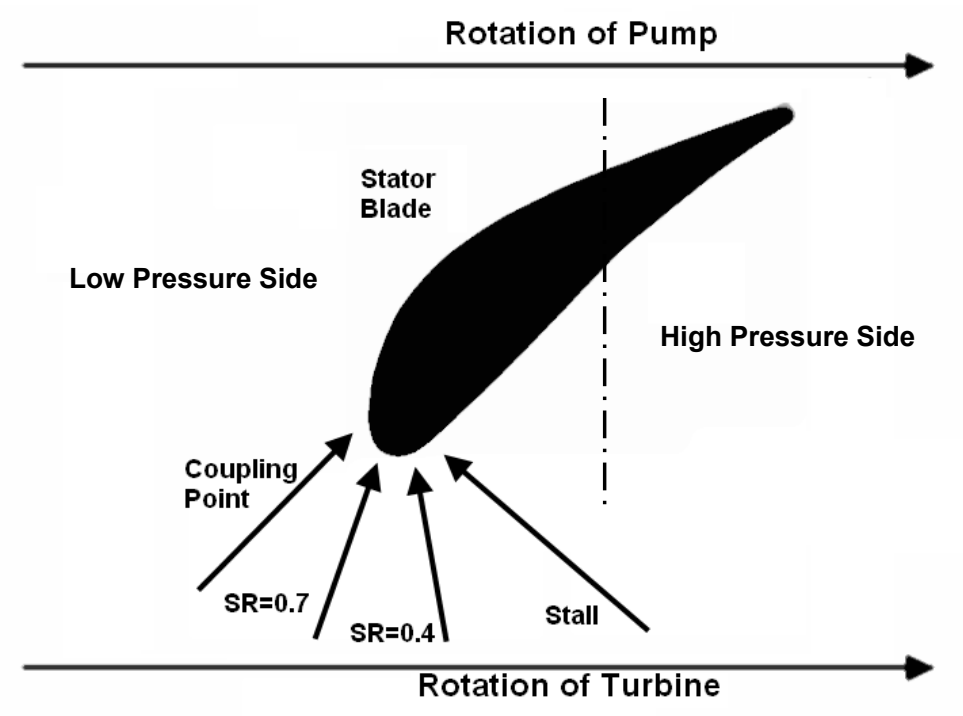

Figure 2.2: Flow incidence angle across the blades of the stator (1)

When the torque converter is operating at high speed ratios, a clutch can be engaged to create a direct shaft connection between the engine and transmission to bypass the hydrodynamic inefficiency of the torque converter. The clutch is engaged by reversing this cooling flow, pushing the pressure plate of the clutch toward the cover of the converter. Apply pressure is regulated to create a full lock between the cover and clutch, or a controlled speed slip.

In torque converter mode, individual element torques are created from the change in angular momentum flux across an element from inlet to outlet. The torque is a function of the local static pressure times the radius integrated over the entire surface of each blade. 
A greater pressure differential across a blade proportionally increases the individual element torque. Consequently, at some large element torque, the pressure on the low pressure side of a blade can drop to below the vapor pressure of the fluid, and the nucleation of cavitation bubbles may occur.

The effect of cavitation on performance is dependent on whether sustained cavitation is reached. Incipient cavitation can occur at stall and dissipate before it is of any consequence. But if a high level of element torque is sustained for a long duration, the heavy cavitation will result in large vapor regions that displace the working fluid, affect performance, and possibly cause damage when cavitation bubbles collapse. Furthermore, collapsing bubbles cause a broadband noise that may affect the overall sound quality of the vehicle. Advanced cavitation causes a decrease in individual element torques, which alters the relationship between speed and torque for the converter.

\subsection{General Considerations}

Torque Converter Cavitation at Stall - In previous research, Robinette et al. (1) and Kowalski et al. (2) studied torque converter cavitation in depth with the converter operating at stall. This is the condition at which a torque converter is most susceptible to cavitation due in part to the high element torques. Toroidal flow within a converter is highly three dimensional with large element flow incidence angles. The high angle of flow into the stator at stall creates regions of separated flow on the low pressure side of the blade. Research by Mekkes et al. (3) confirmed that cavitation initiates in the separated flow region at the stator inlet. As speed ratio increases, stator torque decreases, and flow incidence angle becomes less severe. Regions of separated flow are reduced, and the pressure differential across a stator blade drops, reducing the likelihood of cavitation bubbles forming at the inlet of the stator.

Mekkes (3) used wireless microwave telemetry to measure pressure at the stator inlet. The telemetry was placed on the stator, and pressure measurements were made at the inlet region of the blades. Wireless telemetry was required due to the difficulty in running wires from inside a rotating torque converter to the data acquisition equipment. The telemetry was used to acquire pressure measurements from taps on stator blades and to 
transmit readings to an antenna through the pump via a series of RTV filled slits milled out of the pump/stator interface. This antenna carried the signal to a receiver which converted it back to the raw data for a data acquisition system. A method was developed by Kowalski (2) that uses nearfield acoustic measurements to accurately detect cavitation without requiring wireless telemetry. In this way, a large population of torque converters can be tested for cavitation without the time and expense needed to create microwave instrumented torque converters for each blade design of interest. Robinette et al. (4) used the acoustic method to determine incipient cavitation at stall for a population of torque converters with a wide range of sizes and blade geometries. A correlation was formulated based on operating conditions and converter geometry to estimate the point of incipient cavitation during stall.

Speed Ratio Operation - A torque converter remains in stall only so long as there is not sufficient torque to create vehicle motion. If cavitation is present at stall, it will cease at some higher speed ratio as stator torque and turbine torque decrease. If cavitation at stall is at a low level, and cavitation desinence occurs shortly into the vehicle launch, then noise from cavitation will not be noticed, and its effect on performance will be of no consequence. If, however, torque levels are high enough to sustain cavitation to a high vehicle speed, then cavitation noise could be objectionable to the vehicle operator. Therefore, it is important to characterize the duration of cavitation versus speed ratio with respect to torque converter design parameters and operating conditions. This project utilized the previously developed acoustic method that was used to identify incipient cavitation, but applied it to speed ratio testing to identify cavitation desinence.

\subsection{Experiment}

A dynamometer test fixture was constructed for taking acoustic data with torque converters during the previous stall cavitation project. The fixture is much larger than typically used for torque converter testing so that it can be filled with acoustic foam to assist in absorbing and insulating noise. Figure 2.3 shows pictures of both a typical torque converter test fixture and the acoustically treated fixture used for the cavitation 
experiments. On the right is a picture of the fixture opened to reveal a torque converter and acoustic foam.

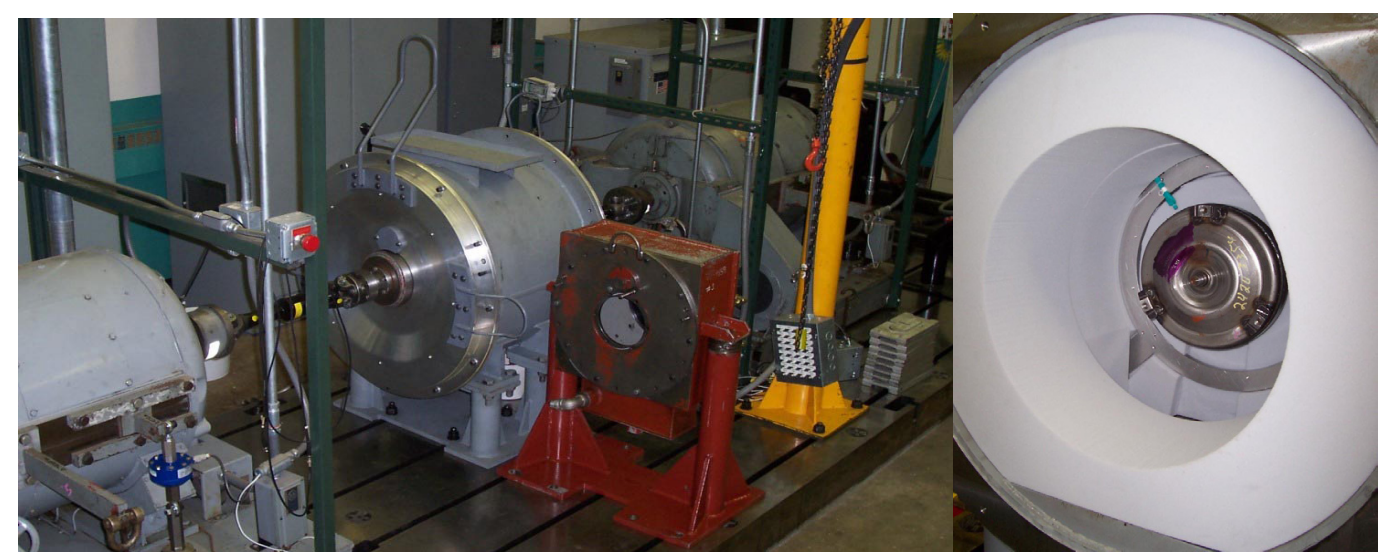

Figure 2.3: Left: Comparison of original torque converter dynamometer test fixture (red) and acoustic test fixture (gray). Right: Close up of opened acoustic test stand with acoustic foam (1)

The SR test procedure was developed to emulate torque converter performance during vehicle takeoff with the engine running at constant torque. During a test, the turbine speed is accelerated at a steady rate while the pump is held at a fixed torque. The first step in the test procedure is to bring the pump up to the torque it will sustain throughout the experiment. A short settling period is needed to allow speed transients due to the control system to settle out. The turbine is then ramped at $50 \mathrm{rpm} / \mathrm{s}$ from low speed (100 $\mathrm{rpm}$ ) to high speed (2500 rpm). An upper speed of $2500 \mathrm{rpm}$ was selected to achieve high enough speed ratio to ensure that cavitation desinence would occur for any of the torque converters planned to be tested. After the test is completed, a torque correction factor which includes dynamic torque losses due to the entire dynamometer system is applied to the measured torques. As with all torque converter testing, speeds, torques, pressures, temperatures, and flow rates are collected via the control system at a $10 \mathrm{~Hz}$ sample rate. Nearfield acoustic measurements are taken at a $51.2 \mathrm{kHz}$ sample rate. Pump and turbine speeds then are also measured at $51.2 \mathrm{kHz}$ so that some reference may be made between the acoustic data and other test parameters.

Speed ratio sweeps were conducted for each torque converter at various input torques and charge pressures. Charge pressures tested were 480, 690, 900, 1030, and $1240 \mathrm{kPa}$ with a 
pressure delta of $-210 \mathrm{kPa}$ for the back pressure. Cooling flow temperature was held constant throughout all of the tests at $70^{\circ} \mathrm{C}$. Input torque for each test was selected based on the torque required to initiate cavitation stall in the previous stall testing plus $70 \mathrm{Nm}$. The extra torque was added to make sure that cavitation occurred during the speed ratio test. The minimum input torque used was $150 \mathrm{Nm}$ and the maximum was $310 \mathrm{Nm}$ for the torque converters in this study.

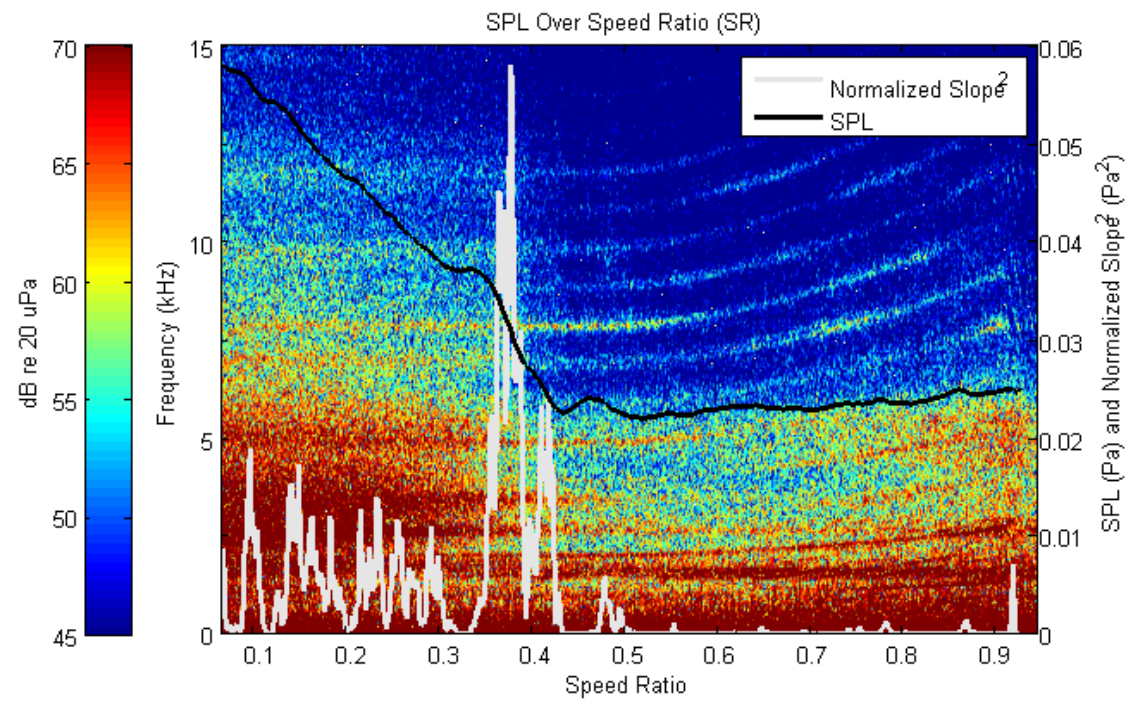

Figure 2.4: Example of torque converter speed ratio data plot

The goal of the speed ratio testing was to determine the desinence of cavitation, or the point at which the bubbles can no longer be detected within the working fluid. In the stall testing by Robinette (1), incipient cavitation was characterized by a sudden increase in the amplitude of the high pass filtered sound pressure level (SPL) of the noise measurements. This SPL was further processed into a slope2 curve, which was then used as an indicator function to determine the onset of cavitation. A similar approach is taken to determine cavitation desinence. Figure 2.4 is a colormap from a SR test showing the nearfield noise versus SR and frequency. Overlaid on the plot are the filtered SPL curve and the slope 2 curve of SPL normalized to the sound pressure level so that both curves can be plotted on the same graph. The slope 2 curve is used to facilitate identifying the key changes in the SPL curve. The region of high broadband noise on the left half side of the colormap corresponds to cavitation. The high magnitude peak in the slope 2 curve near 0.4 SR corresponds to the desinence of cavitation. 


\subsection{Analysis}

Preliminary Data - After data for a torque converter SR test is acquired, the speed ratio at desinence of cavitation, labeled $\mathrm{SR}_{\mathrm{d}}$, needs to be identified. During speed ratio operation, however, the noise from the turbine and pump increases as turbine speed increases, and the overall noise content of the converter is complex. This is evidenced by the numerous local peaks and valleys in the SPL curve in Figure 2.4. SPL drops to a fairly constant level after cavitation stops, but the roughness of the curve and the lack of a sharp transition from the cavitation region to non cavitation necessitated the development of a repeaTable 2.and objective procedure for identifying $\mathrm{SR}_{\mathrm{d}}$. Figure 2.5 demonstrates the method that was used.
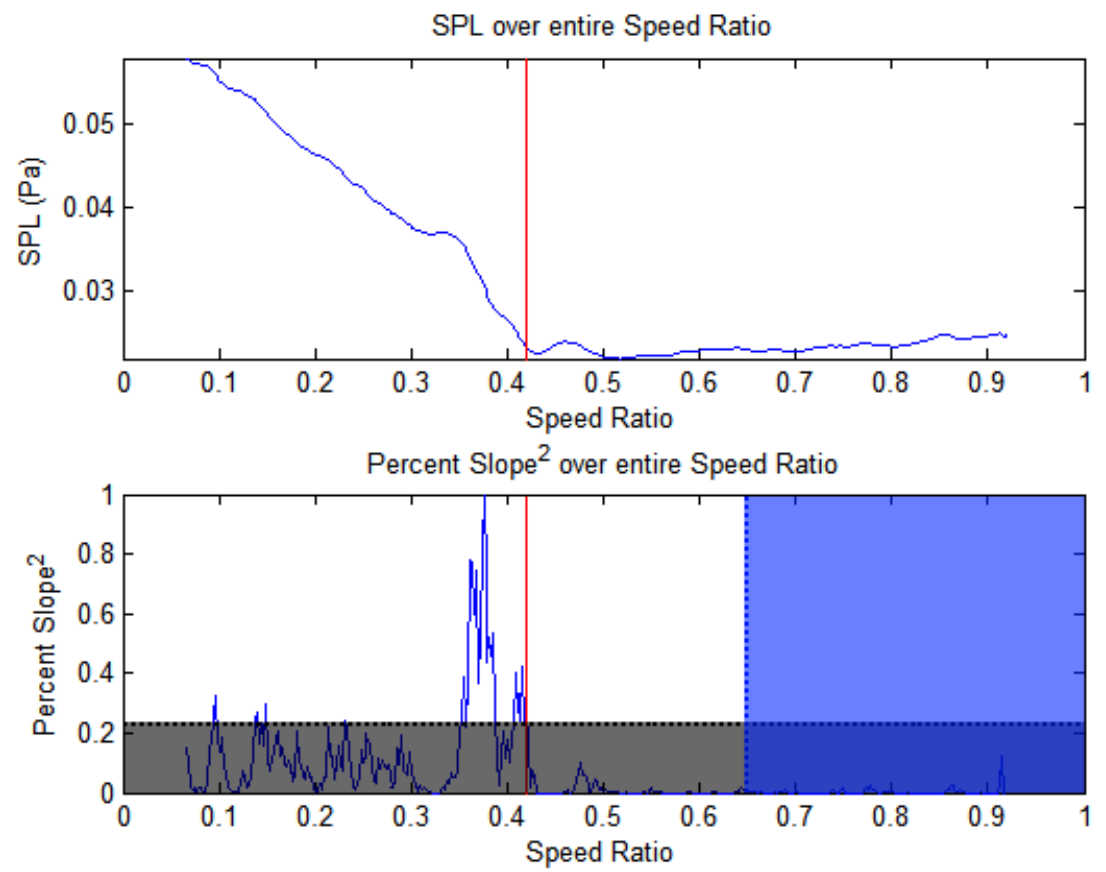

Figure 2.5: Visualization of the algorithm used to determine desinent cavitation in a torque converter

The top portion of Figure 2.5 shows the filtered SPL curve. A high pass filter with a cutoff of $10 \mathrm{kHz}$ is used to filter out the background noise, rotational orders, and blade pass frequencies that occur below $10 \mathrm{kHz}$. The resulting filtered SPL curve displays the wideband noise from cavitation. As expected, cavitation noise is highest near stall, and gradually decreases to a relatively constant level when cavitation bubbles cease to form. A lower high pass filter of $6 \mathrm{kHz}$ was used in the analysis by Robinette (1) due to less 
background noise being present in stall testing. Due to the jagged nature of the SPL curve, it is smoothed using a three point running average filter. This curve is then used to generate a slope 2 curve plotted as a percentage as shown in the bottom portion of Figure 2.5. The resulting curve is examined to find the maximum speed ratio less than 0.65 where the slope 2 curve is greater than 22.5 percent of its maximum. The $0.65 \mathrm{SR}$ limit was determined after examining data from all the tests and finding that cavitation always ends at a lower speed ratio. The 22.5 percent slope2 threshold was selected after studying the data from all the tested converters and selecting a level that correlated with the perceived change in the SPL over the entire population. These criteria were entered into an algorithm to search for the points of cavitation desinence from all the test runs. Figure 2.5 shows the resulting slope 2 curve from the colormap in Figure 2.4 with the excluded areas shaded out in the lower plot, and the determined point of desinent on both plots. The fluid parameters required for further analysis are taken at the speed ratio of cavitation desinence.

Dimensional Analysis - Dimensional analysis was used to develop models for predicting torque converter cavitation desinence using torque converter design parameters and operating parameters measured or controlled in the speed ratio tests. In the stall cavitation research done by Robinette (4), a set of dimensionless parameters that incorporates torque converter design variables, performance parameters and operating conditions was derived and used to develop dimensionless models to predict the emergence of cavitation at stall. Similar parameters were used in this study to develop models for cavitation desinence, but were modified somewhat to accommodate speed ratio operation. These are listed in Table 2.1. 
Table 2.1: Dimensionless Parameters used in Speed Ratio Correlation

\begin{tabular}{|c|c|c|}
\hline$\prod_{1}=U_{\text {stall }}$ & $\begin{array}{l}\text { Unit Input } \\
\text { Speed }\end{array}$ & $\begin{array}{l}\text { Based on overall torque } \\
\text { converter design }\end{array}$ \\
\hline$\prod_{2}=T R_{\text {stall }}$ & $\begin{array}{l}\text { Stall Torque } \\
\text { Ratio }\end{array}$ & $\begin{array}{l}\text { Based on overall torque } \\
\text { converter design }\end{array}$ \\
\hline$\prod_{3}=\frac{L_{t}}{D}$ & $\begin{array}{l}\text { Torus Aspect } \\
\text { Ratio }\end{array}$ & $\begin{array}{l}\text { Based on torque converter } \\
\text { geometry }\end{array}$ \\
\hline$\prod_{4}=\frac{t_{\max }}{l_{c}}$ & $\begin{array}{l}\text { Stator Blade } \\
\text { Thickness } \\
\text { Ratio }\end{array}$ & $\begin{array}{l}\text { Based on torque converter } \\
\text { geometry }\end{array}$ \\
\hline$\prod_{5}=N_{s b}$ & $\begin{array}{l}\text { Number of } \\
\text { Stator Blades }\end{array}$ & $\begin{array}{l}\text { Based on torque converter } \\
\text { geometry }\end{array}$ \\
\hline$\prod_{6}=\frac{\Delta p}{p_{\text {ave }}}$ & $\begin{array}{l}\text { Dimensionless } \\
\text { Pressure Ratio }\end{array}$ & Operating Point Parameter \\
\hline$\prod_{7}=\operatorname{Pr}$ & $\begin{array}{l}\text { Prandtl } \\
\text { Number }\end{array}$ & Operating Point Parameter \\
\hline$\prod_{r e s p}=\frac{T_{p}}{D^{3} p_{a v e}} * S R_{d}$ & $\begin{array}{l}\text { Dimensionless } \\
\text { Rotational } \\
\text { Power }\end{array}$ & $\begin{array}{l}\text { Combination of Operating } \\
\text { Point Parameter and Measured } \\
\text { Response }\end{array}$ \\
\hline
\end{tabular}

Unit input speed at stall, $\mathrm{U}$, shown in Equation 1, is a parameter that describes torque converter performance and is used to select the best torque converter for a given engine and vehicle application. It contains the relationship between the working fluid (density), the size of the converter (diameter), the input speed, and the input torque.

$$
U=N_{p}\left(\frac{\rho D^{5}}{T_{p}}\right)^{\frac{1}{2}}
$$

The value of $U$ is a function of the blade designs for the pump, turbine, and stator. It is used in the dimensional analysis instead of using a longer list of blade design parameters. 
Torque Ratio was described earlier as the ratio of output torque over input torque. Note that since both $U$ and TR have a dependence on speed ratio, the values of these variables at stall were used for this correlation to improve equation conditioning.

Torus aspect ratio is a definition of the roundness of a torque converter. The measurements used for this along with stator blade thickness ratio are shown in Figure 2.6. The number of stator blades is a design variable that is a function of stator blade angles and the axial length of the stator.

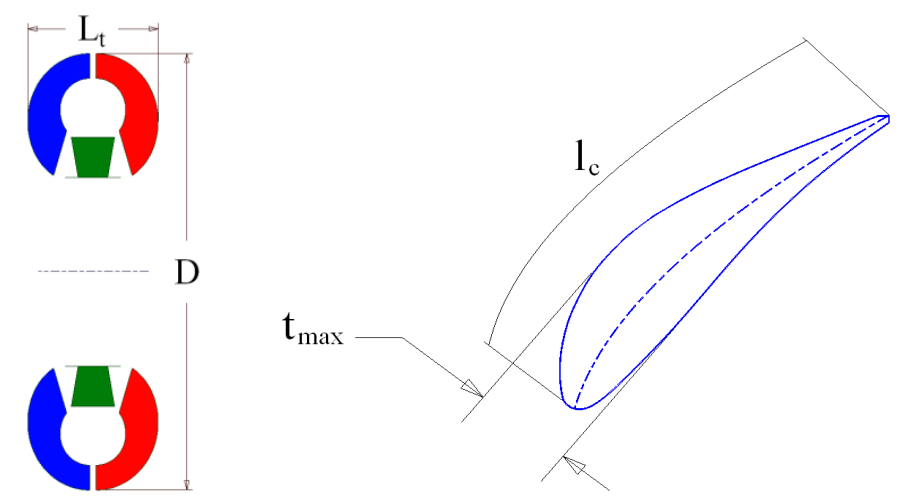

Figure 2.6: Left: Schematic of torque converter showing diameter (D) and torus length $\left(L_{t}\right)$ Right: Profile of stator blade depicting maximum thickness $\left(t_{\max }\right)$ and chord length $\left(I_{c}\right)(1)$

The dimensionless pressure ratio and the Prandtl number are calculated from the values of the measured charge and back pressures, flows, and temperature taken due to the cooling flow mentioned in the introduction. The formulations of these parameters are shown in Equations 2 and 3. In Equation 2, $\mathrm{p}_{\text {charge }}$ and $\mathrm{p}_{\text {back }}$ are the charge and back pressures applied to the test stand during operation respectively. The term, $c_{p}$, in Equation 3, is the specific heat of the working fluid, $\mathrm{k}$ is the thermal conductivity, and $\mu$ is the dynamic viscosity. All of these values are measured or calculated based on the temperature at the point of cavitation desinence.

$$
\begin{gathered}
\frac{\Delta p}{p_{\text {ave }}}=\frac{2\left(p_{\text {charge }}-p_{\text {back }}\right)}{\left(p_{\text {charge }}+p_{\text {back }}\right)} \\
\operatorname{Pr}=\frac{c_{p} \mu}{k}
\end{gathered}
$$


The last dimensionless group, Dimensionless Rotational Power, is the product of the speed ratio at desinent cavitation, $\mathrm{SR}_{\mathrm{d}}$, (selected by the algorithm detailed in Section 3) and the dimensionless input torque. This combination of dimensionless parameters was selected as the dimensionless response in the correlation development because it is defined by quantities that can be used to assess the potential for cavitation in a new torque converter design or a new application of an existing design.

The dimensionless groups are applied to a Response Surface Model (RS) using a stepwise regression procedure to predict the dimensionless rotational power. The general form of the response surface model is outlined in the research of Madsen et al. (6), and is given here by Equation 4 .

$$
\hat{y}=\beta_{0}+\sum_{i=1}^{k} \beta_{i} x_{i}+\sum_{i=1}^{k} \beta_{i i} x_{i}^{2}+\sum_{i=1}^{k-1} \sum_{j=i+1}^{k} \beta_{i j} x_{i} x_{j}+\epsilon
$$

The response, $\hat{y}$, is dimensionless rotational power, while the linear, quadratic, and interaction terms are generated from the other dimensionless regressors listed in Table 2.1. These are used to solve for the $\beta_{0}, \beta_{\mathrm{ii}}$, and $\beta_{\mathrm{ij}}$ terms using a least squares method. The residual error, symbolized in Equation 4 as $\varepsilon$, is the difference between the experimentally measured quantity of the dimensionless rotational power and the predicted value.

A forward selection path of stepwise regression is used to minimize the error in the models. This method starts with a basic equation comprised of only the intercept term, $\beta_{0}$. From there, regressors are added until the error of the model is minimized. At each step the response is calculated using all of the remaining regressors in addition to those that had previously been added, after which the new regressor is selected by determining which one minimizes the error to the greatest degree.

The quality of fit for any RS model is verified by the root mean square error (RMSE) and by determining the linear association between the dimensionless response and the regressors. RMSE estimates the model's standard deviation and for these correlations are computed as a percentage. Equation 5 shows the calculation for \%RMSE. The term yi is 
the dimensionless rotational power, $\hat{y} i$ is the calculated response from the model, $\mathrm{n}$ is the number of data points, and $\mathrm{p}$ is the number of dimensionless regressors in the model.

$$
\% R M S E=\sqrt{\frac{\sum_{i=1}^{n}\left(\left(\left(y_{i}-\hat{y}_{i}\right) / y_{i}\right) * 100\right)^{2}}{n-p}}
$$

The proportionate amount of variation in the response explained by a particular set of regressors in the model is measured using the adjusted coefficient of multiple determination, $\mathrm{R}_{\mathrm{a}}^{2}$, in Equation 6. This metric calculates to between 0 and 1. Generally, results above .85 signify an accurate model of the data in question. The coefficient of multiple determination, $\mathrm{R}^{2}$, was also calculated. However, it is not preferred as the value of $\mathrm{R}^{2}$ always increases with the addition of regressors. $\mathrm{R}^{2}$ may increase or decrease depending on whether or not the additional terms actually reduce variation in the response.

$$
R_{a}^{2}=1-\frac{\sum_{i=1}^{n}\left(y_{i}-\hat{y}_{i}\right) / n-p}{\sum_{i=1}^{n}\left(y_{i}-\bar{y}\right) / n-1}
$$

Figure 2.7 shows results from the stepwise regression procedure for the analysis of a population of round torus torque converters to determine the regressors to be added to the model. Each step of the procedure optimizes the $\%$ RMSE, the $\mathrm{R}^{2}$, or both. Only those regressors that have a significant impact on model error or linear association between the response and the regressors are included in the model. This makes the final model for the example population a function of 16 terms, including the intercept term, instead of the 36 possible terms. The value for $\mathrm{R}^{2}$ a was brought to above .85 within the first three iterations. Each subsequent iteration was done to improve model error. 


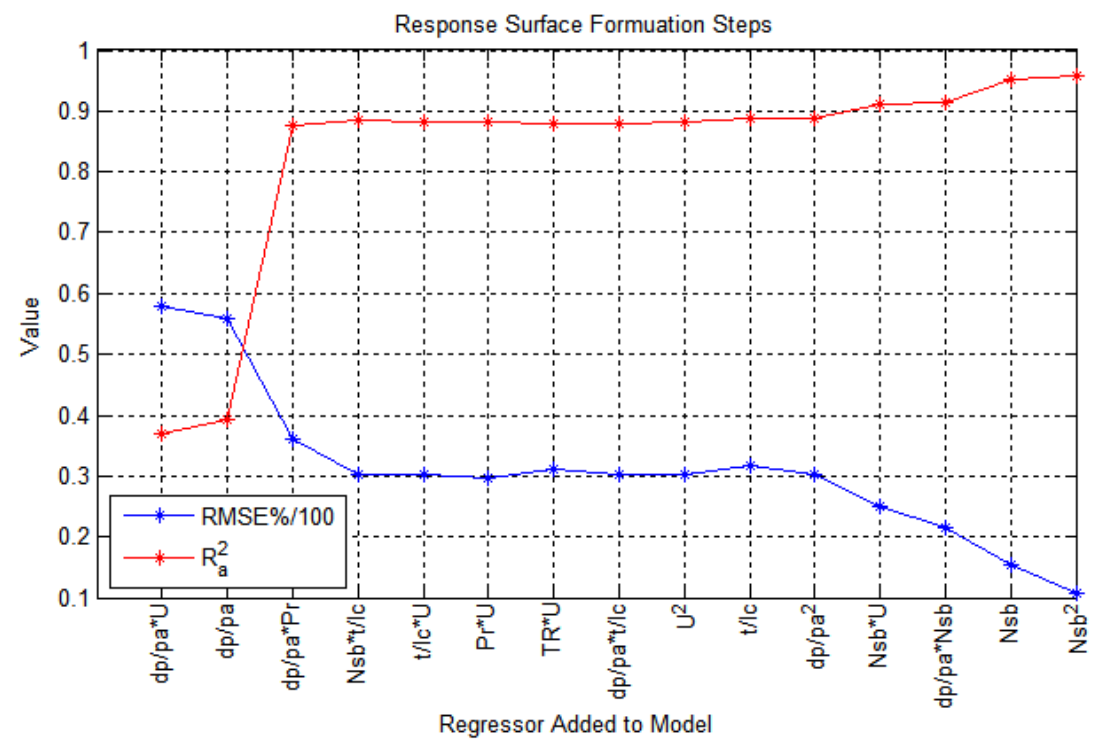

Figure 2.7: Reduction of \%RMSE and $\mathrm{R}^{2}$ a during stepwise regression for round torus model

Conditioning of the data matrix also needs to be taken into account. This study was done with a limited number of converters, some of which were somewhat similar. With an illconditioned system, if too many regressors are added to the formulation for a particular family of converters, the data matrix becomes close to singular due to the fact that some of the data being used may be similar. The resulting model is unsTable 2.in that small changes in the dimensionless regressors will create very large changes with respect to the response.

\subsection{Results}

Several RS models based on geometric similitude between torque converters are presented. Each group increases in complexity as the similarity between the converters in the family decreases. The first group shows a correlation using converters of the same unit input speed. The second deals with a specific torus aspect ratio which is common to the converters in that group. As such, that dimensionless regressor is eliminated from the correlation. The third group, made up of machined from solid only torque converters, was analyzed to determine if geometric differences that result from the manufacturing process have an effect on formulating a response surface model. The last group correlates all of the converters tested that were made by the same manufacturer. The torque converters in 
the final group cover a wide range of geometry, but follow similar design rules for torus shapes, blade angles, and for the stator blades, the airfoil shapes. The goal was to develop a general model for predicting dimensionless rotational power at cavitation desinence that can be applied to a wide range of torque converter designs. The predicted value can be used with input torque and operating parameters for a specific application to calculate $\mathrm{SR}_{\mathrm{d}}$ and estimate the risks for cavitation.

Constant Unit Input Speed - Three torque converters with close to the same unit input speed, but different diameters were analyzed in this group to model how converters with similar unitized performance characteristics compare with regards to desinent cavitation. The functional form of this RS model is given in Equation 7. Figure 2.8 is a plot of dimensionless rotational power at cavitation desinence that compares test results (point data) with the predicted curve from the model.

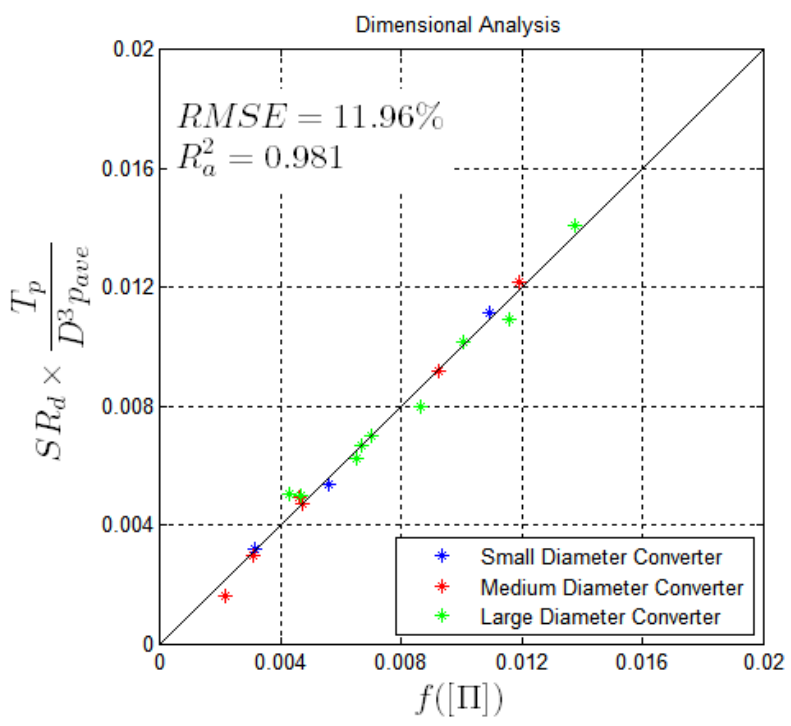

Figure 2.8: RS model of desinent cavitation in constant unit input speed family

$$
f\left(U_{\text {stall }}, T R_{\text {stall }}, \frac{L_{t}}{D}, N_{s b}, \frac{\Delta p}{p_{\text {ave }}}, \operatorname{Pr}\right)
$$

The \%RMSE and $\mathrm{R}_{\mathrm{a}}^{2}$ values for the RS model are also displayed in Figure 2.8. The model uses a total of 9 terms that are a combination of the dimensionless regressors listed in Equation 7 plus $\beta_{0}$ to determine the estimated dimensionless rotational power. This population contains three torque converters, with a total of 18 test conditions. The resulting RS model can be used to predict dimensionless rotational power at cavitation 
desinence with less than $12 \%$ RMSE for torque converter designs with this unit input speed. It should be noted that although the values for unit input speed for the three converters in this population are very close, they are not identical.

Round Torus - The next family contains torque converters with a large torus aspect ratio, considered to be round in shape. As similarity of torus ratio exists between all of these converters, that regressor was ignored in computing the correlation. This population contains six torque converters with a total of 49 test conditions. The converters are comprised of a range of component blade designs. Due to greater design diversity as compared to the constant unit input speed population, more terms, a total of 16 , are required to minimize the model error. Figure 2.9 shows the model diagnostics, as well as the plot of measured response versus response calculated from regressors. Equation 8 is the functional form of the RS model. The model from this group can be used to predict dimensionless rotational power for torque converters with similar torus aspect ratio as this population with less than 11 percent error.

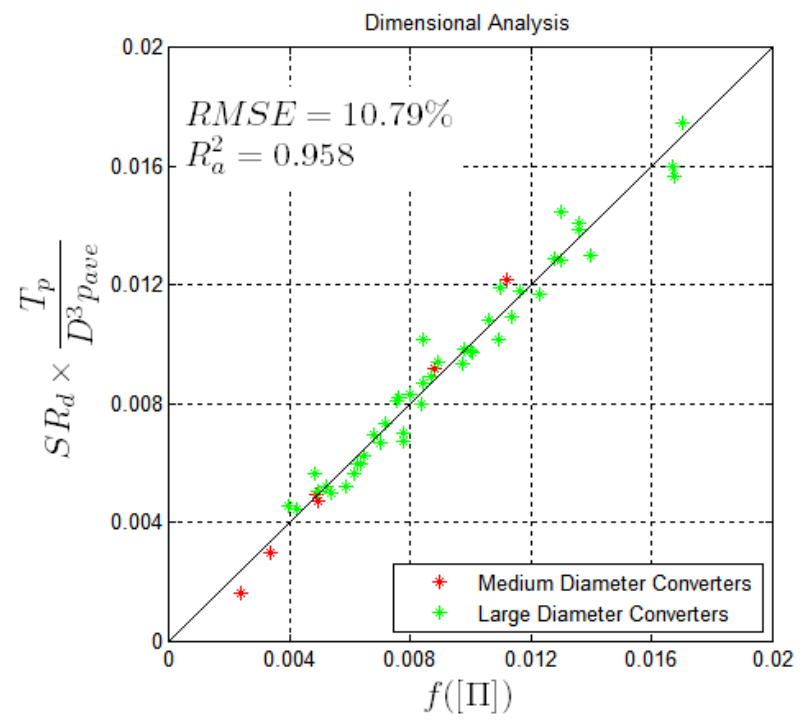

Figure 2.9: RS model of desinent cavitation in torque converters with a round torus shape

$$
\frac{T_{p}}{D^{3} p_{\text {ave }}} * S R_{d}=f\left(U_{\text {stall }}, T R_{\text {stall }}, \frac{t_{\text {max }}}{l_{c}}, N_{s b}, \frac{\Delta p}{p_{\text {ave }}}, P r\right)
$$

Similar to the case with the unit input speed population, there was some small variation in the torus aspect ratio with this group. However, because that regressor did not appear 
dominant when making this formulation, it was omitted from the final response calculation.

Machined From Solid Torque Converters - The population of the RS model shown in Figure 2.10 is made up of torque converters with machined from solid (MFS) pump, turbine, and stator assemblies. The MFS components are one off prototypes with tight size tolerances and sealed flow passages. Production pumps and turbines are made from stamped sheet metal blades and housings, and can have small leak paths between blades. MFS stator blades surfaces have a smoother surface finish than blades from a typical production cast stator.

The population for this model contained six torque converters and 46 test conditions. A total of 17 terms, comprised of all the possible dimensionless regressors and $\beta_{0}$, were used to calculate the response in this model. The torque converters in this group were made up of a range of diameters, torus aspect ratios, and blade designs such that all of the dimensionless regressors are significant to the model. Equation 9 shows the functional from of the response surface model.

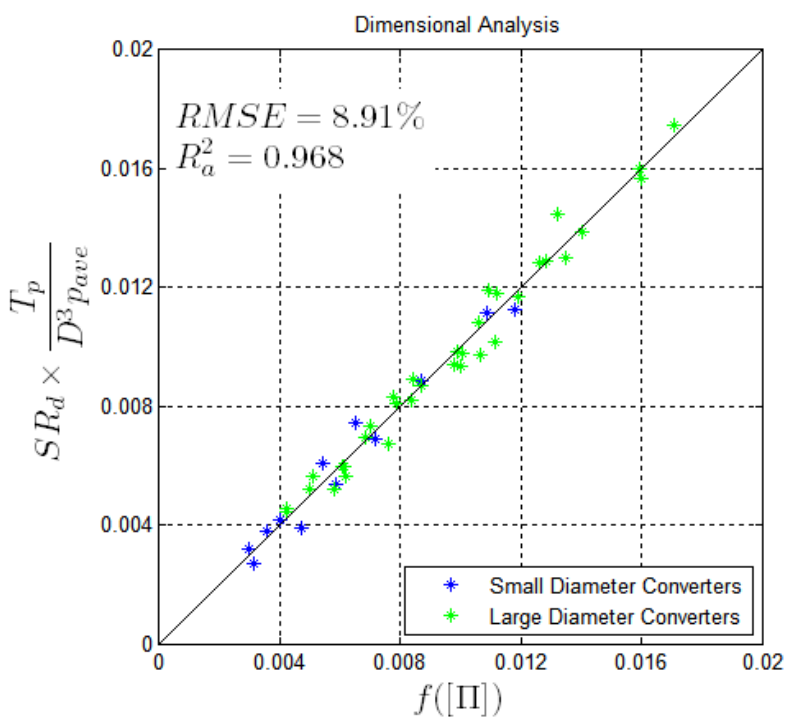

Figure 2.10: RS model of desinent cavitation in machined from solid torque converters

$$
\frac{T_{p}}{D^{3} p_{\text {ave }}} * S R_{d}=f\left(U_{\text {stall }}, T R_{\text {stall }}, \frac{L_{t}}{D}, \frac{t_{\text {max }}}{l_{c}}, N_{\text {sb }}, \frac{\Delta p}{p_{\text {ave }}}, \operatorname{Pr}\right)
$$


Although this group of converters is more diverse than the previously analyzed families, the accuracy with which the model can predict desinence is better. This might be due to the manufacturing differences between MFS components and production stamped and cast components. There can be part to part variation in the leak paths between blades and housings for stamped pumps and turbines which is eliminated with MFS parts. This can impact variations in unit input speed and torque ratio performance. Similarly with cast stators, there is greater part to part variation of blade surface finish which could affect nucleation sites for cavitation bubbles. Both of these features may contribute to the reason why the RS analysis for this family of converters converged to a model that has more accuracy as compared to the others.

Entire Population - Figure 2.11 shows the response surface model developed for the entire tested torque converter population. Eight torque converters and 61 test conditions were used to develop the generalized RS model. Similar to the MFS population, all dimensionless regressors were included in Equation 9 because of the variety of geometries and sizes in the population. The difference in this group from the previous is that it includes a combination of production and MFS assemblies. The final RS model contains 21 terms.

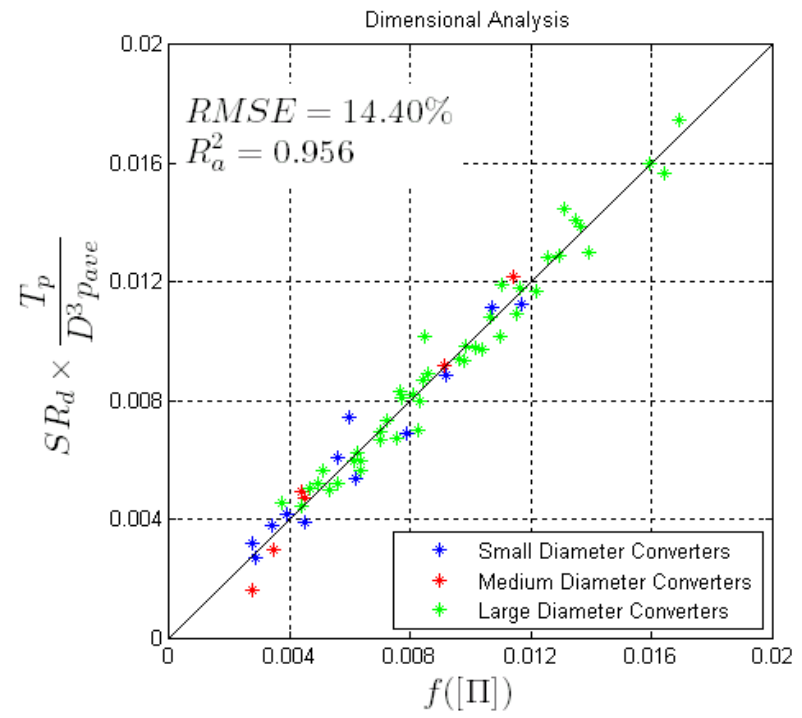

Figure 2.11: RS model of desinent cavitation in entire torque converter population

$$
\frac{T_{p}}{D^{3} p_{\text {ave }}} * S R_{d}=f\left(U_{\text {stall }}, T R_{\text {stall }}, \frac{L_{t}}{D}, \frac{t_{\text {max }}}{l_{c}}, N_{\text {sb }}, \frac{\Delta p}{p_{\text {ave }}}, \operatorname{Pr}\right)
$$


The RS model for this group essentially creates a generalized model to calculate the point of desinent cavitation of a converter during speed ratio operation. Equation 10 shows the functional form of the correlation for the entire converter population tested. The RS model can be used to predict dimensionless rotational power at desinent cavitation with $14.4 \%$ RMSE for torque converters that follow similar design rules for torus shapes and blade designs as those that were tested.

Model Discussion - A summary of the RS model equations for all four groups minus regression coefficients is listed in Table 2.2. The number of regression terms required to achieve desired accuracy increases with wider ranges of geometric design and performance parameters due to more interaction between parameters. All of the RS model equations include contributions from operating parameter and design parameter regressors. 
Table 2.2: RS Models by Torque Converter Population minus Regression Coefficients

\begin{tabular}{|c|c|}
\hline Population & Response surface model \\
\hline $\begin{array}{l}\text { Constant Unit Input } \\
\text { Speed }\end{array}$ & $\begin{array}{r}\frac{T_{p}}{D^{3} p_{\text {ave }}} * S R_{d}=\beta_{0}+\beta_{1}\left(\frac{\Delta p}{p_{\text {ave }}}\right)\left(T R_{\text {stall }}\right)+\beta_{2}\left(U_{\text {stall }}\right)^{2} \cdots \\
\cdots+\beta_{3}\left(\frac{\Delta p}{p_{\text {ave }}}\right)(\operatorname{Pr})+\beta_{4}\left(\frac{\Delta p}{p_{\text {ave }}}\right)+\beta_{5}\left(\frac{\Delta p}{p_{\text {ave }}}\right)^{2}+\beta_{6}(\operatorname{Pr})\left(N_{\text {sb }}\right) \ldots \\
\cdots+\beta_{7}\left(\frac{t_{\text {max }}}{l_{c}}\right)\left(\frac{L_{t}}{D}\right)+\beta_{8}\left(N_{s b}\right)\left(\frac{L_{t}}{D}\right)\end{array}$ \\
\hline Round Torus & $\begin{array}{r}\frac{T_{p}}{D^{3} p_{\text {ave }}} * S R_{d}=\beta_{0}+\beta_{1}\left(\frac{\Delta p}{p_{\text {ave }}}\right)\left(U_{\text {stall }}\right)+\beta_{2}\left(\frac{\Delta p}{p_{\text {ave }}}\right)+\beta_{3}\left(\frac{\Delta p}{p_{\text {ave }}}\right)(\operatorname{Pr}) \ldots \\
\cdots+\beta_{4}\left(N_{\text {sb }}\right)\left(\frac{t_{\text {max }}}{l_{c}}\right)+\beta_{5}\left(\frac{t_{\text {max }}}{l_{c}}\right)\left(U_{\text {stall }}\right)+\beta_{6}(\operatorname{Pr})\left(U_{\text {stall }}\right) \ldots \\
\ldots+\beta_{7}\left(T R_{\text {stall }}\right)\left(U_{\text {stall }}\right)+\beta_{8}\left(\frac{\Delta p}{p_{\text {ave }}}\right)\left(\frac{t_{\text {max }}}{l_{c}}\right)+\beta_{9}\left(U_{\text {stall }}\right)^{2} \ldots \\
\cdots+\beta_{10}\left(\frac{t_{\text {max }}}{l_{c}}\right)+\beta_{11}\left(\frac{\Delta p}{p_{\text {ave }}}\right)^{2}+\beta_{12}\left(N_{\text {sb }}\right)\left(U_{\text {stall }}\right) \ldots \\
\cdots+\beta_{13}\left(\frac{\Delta p}{p_{\text {ave }}}\right)\left(N_{\text {sb }}\right)+\beta_{14}\left(N_{\text {sb }}\right)+\beta_{15}\left(N_{\text {sb }}\right)^{2}\end{array}$ \\
\hline Machined from Solid & $\begin{array}{r}\frac{T_{p}}{D^{3} p_{\text {ave }}} * S R_{d}=\beta_{0}+\beta_{1}\left(\frac{\Delta p}{p_{\text {ave }}}\right)\left(\frac{L_{t}}{D}\right)+\beta_{2}\left(U_{\text {stall }}\right)^{2}+\beta_{3}(\operatorname{Pr})\left(U_{\text {stall }}\right) \ldots \\
\cdots+\beta_{4}\left(T R_{\text {stall }}\right)^{2}+\beta_{5}(\operatorname{Pr})^{2}+\beta_{6}\left(\frac{L_{t}}{D}\right)\left(U_{\text {stall }}\right)+\beta_{7}\left(\frac{\Delta p}{p_{\text {ave }}}\right)\left(U_{\text {stall }}\right) \ldots \\
\cdots+\beta_{8}\left(\frac{\Delta p}{p_{\text {ave }}}\right)\left(\frac{t_{\text {max }}}{l_{c}}\right)+\beta_{9}\left(\frac{\Delta p}{p_{\text {ave }}}\right)(\operatorname{Pr})+\beta_{10}\left(\frac{\Delta p}{p_{\text {ave }}}\right)\left(T R_{\text {stall }}\right)+\beta_{11}(\operatorname{Pr}) \ldots \\
\cdots+\beta_{12}(\operatorname{Pr})\left(\frac{L_{t}}{D}\right)+\beta_{13}\left(T R_{\text {stall }}\right)\left(U_{\text {stall }}\right)+\beta_{14}\left(T R_{\text {stall }}\right) \ldots \\
\cdots+\beta_{15}\left(U_{\text {stall }}\right)+\beta_{16}\left(N_{\text {sb }}\right)\left(\frac{L_{t}}{D}\right)\end{array}$ \\
\hline Entire Population & $\begin{array}{r}\frac{T_{p}}{D^{3} p_{\text {ave }}} * S R_{d}=\beta_{0}+\beta_{1}\left(\frac{\Delta p}{p_{\text {ave }}}\right)+\beta_{2}\left(\frac{\Delta p}{p_{\text {ave }}}\right)(P r)+\beta_{3}\left(\frac{\Delta p}{p_{\text {ave }}}\right)\left(U_{\text {stall }}\right) \ldots \\
\cdots+\beta_{4}\left(\frac{L_{t}}{D}\right)^{2}+\beta_{5}\left(\frac{L_{t}}{D}\right)+\beta_{6}\left(\frac{\Delta p}{p_{\text {ave }}}\right)\left(N_{\text {sb }}\right)+\beta_{7}\left(\frac{L_{t}}{D}\right)(\operatorname{Pr}) \ldots \\
\cdots+\beta_{8}\left(T R_{\text {stall }}\right)\left(\frac{L_{t}}{D}\right)+\beta_{9}\left(\frac{t_{\text {max }}}{l_{c}}\right)\left(N_{\text {sb }}\right)+\beta_{10}\left(U_{\text {stall }}\right)^{2}+\beta_{11}\left(\frac{\Delta p}{p_{\text {ave }}}\right)^{2} \cdots \\
\cdots+\beta_{12}\left(U_{\text {stall }}\right)+\beta_{13}\left(U_{\text {stall }}\right)\left(T R_{\text {stall }}\right)+\beta_{14}\left(\frac{\Delta p}{p_{\text {ave }}}\right)\left(T R_{\text {stall }}\right) \ldots \\
\cdots+\beta_{15}\left(T R_{\text {stall }}\right)^{2}+\beta_{16}\left(U_{\text {stall }}\right)\left(N_{\text {ssb }}\right)+\beta_{17}\left(\frac{L_{t}}{D}\right)\left(N_{s b}\right) \ldots \\
\cdots+\beta_{18}\left(N_{\text {ssb }}\right)(P r)+\beta_{19}\left(U_{\text {stall }}\right)\left(\frac{t_{\text {max }}}{l_{c}}\right)+\beta_{20}\left(T R_{\text {stall }}\right)\left(N_{\text {sb }}\right)\end{array}$ \\
\hline
\end{tabular}

In contrast to the models developed by Robinette (4) to predict incipient cavitation at stall, the models for cavitation desinence have larger values for \%RMSE. One contributor could be that the torque converters in the stall cavitation study were all comprised of MFS components. The RS model for the MFS population in this study has the highest accuracy of any of the groups. Greater model accuracy could be from less variation in $\mathrm{SR}_{\mathrm{d}}$ for a family of MFS converters due to reduction in part to part variation in blade 
surface finish for MFS stators as compared to die cast and to the elimination of small blade to housing flow paths in MFS pumps and turbines.

The turbine speed sweep rate might also be a factor in model accuracy. While the turbine acceleration that was used in dynamometer testing is not as fast as in a typical vehicle at wide open throttle, a slower sweep rate might reduce errors from the transient response of the torque converter dynamometer. Additional speed ratio testing at different rates could be done to measure potential influence.

\subsection{Conclusions}

A method was developed to determine the speed ratio of cavitation desinence in automotive torque converters by testing on a dynamometer and using acoustic measurements. Desinent cavitation for torque converters of various geometries was determined, and corresponding data was used to formulate RS models by which it can be predicted. The torque converter geometries and operating parameters of the working fluid were used as dimensionless regressors, while dimensionless rotational power was used as the dimensionless response. A stepwise regression analysis was used to simplify each model so that only the regressors that significantly reduced the error in the model while enhancing the model's predictive capability were included.

Test results from several populations of torque converters were analyzed to create RS models. As populations increase in diversity, the models are more complex. The RS models that were created based on the converters having similar geometric properties (Torus aspect ratio) or similar operating capacities (Unit input speed) are capable of predicting the dimensionless rotational power of the converter at the point of desinent cavitation within a \%RME of 10.8 and 11.6 percent respectively. The general model developed from test results for all the converters tested is capable of predicting dimensionless rotational power at desinent cavitation with $14.4 \% \mathrm{RMSE}$. The $\mathrm{R}^{2}$ a of all models presented is greater than .85 , making each of them useful as an accurate model to estimate the dimensionless rotational power. 


\subsection{References}

(1) Robinette DL. Detecting and Predicting the onset of Cavitation in Automotive Torque Converters. PhD Dissertation, Michigan Technological University. Ann Arbor: ProQuest/UMI. 2007.

(2) Kowalski D, Anderson CL, Blough JR. Cavitation Detection in Automotive Torque Converters Using Nearfield Acoustical Measurements. SAE-NVH. 5/16/2005; 200501-2516.

(3) Mekkes J, Anderson CL, Narain A. Static Pressure Measurements on the Nose of a Torque Converter Stator during Cavitation. ISROMAC. 2004.

(4) Robinette DL, Schweitzer JM, Maddock DG, Anderson CL, Blough JR, Johnson MA. Predicting the Onset of Cavitation in Automotive Torque Converters - Part II: A Generalized Model. International Journal of Rotating Machinery. 2008; Article ID 312753.

(5) Dong Y, Lakshminarayana B. Rotating Probe Measurements of the Pump Passage Flow Field in an Automotive Torque Converter. ASME J. of Fluids Eng.. 2001; Volume 123: pp. 81-91.

(6) Madsen JI, Shyy W, Haftka RT. Response Surface Techniques for Diffuser Shape Optimization. AIAA Journal. 2000; Volume 38, No 9: pp. 1512-1518.

(7) Strasberg M, Taylor D. Propeller Cavitation Noise after 35 Years of Study. Noise and Fluids Engineering. 1977.

(8) Robinette DL, Anderson CL, Blough JR, Johnson MA, Maddock DG, Schweitzer JM. Characterizing the Effect of Automotive Torque Converter Design Parameters on the Onset of Cavitation at Stall. SAE-NVH. 5/15/2007; 2007-01-2231. 


\section{Chapter 3 - Measuring and Comparing Frequency Response Functions of Torque Converter Turbines Submerged in Transmission Fluid $^{1}$}

C. Walber, J. Blough, C. Anderson, M. Johnson

Michigan Technological University, Department of Mechanical Engineering

1400 Townsend Dr. Houghton, Michigan, USA

Copyright $(\underset{0}{ } 2011$ SAE International

\subsection{Abstract}

When testing dynamic structures, it is important to note that the dynamic system in question may be submerged into a fluid during operation and to properly test the structure under the same condition in order to understand the true dynamic parameters of the system. In this way, the mass and stiffness coupling to the particular fluid, for the case of this study, automatic transmission fluid, may be taken into account. This is especially important in light structures where the coupling between the fluid mass and the structural mass may be great. A structure was tested with a laser vibrometer using several impact methods in open air to determine which impact method would be most suiTable 3.for submerged testing. The structure was then submerged in transmission fluid with an accelerometer attached and subsequently tested and compared to the previous results. In addition, two novel means of excitation, by use of a ball bearing impact and by use of a weighted impact rod, will also be discussed and compared.

\footnotetext{
${ }^{1}$ The material contained in this chapter has been published in the Journal of SAE Noise and Vibration
} 


\subsection{Introduction}

Structural dynamics experimentation has evolved greatly over the years. An experimentalist is called upon to adapt to various situations, including testing buildings, vehicles, individual parts, or even machinery as it is operating. Each situation requires a different approach to acquire the information needed to understand the system in question. However, even with the many commercially available test products it may not be able to test a structure in its operational environment. The structure is often suspended in a near free-free state, transducers are often affixed to the structure that may cause mass loading effects that can be detectible in the final calculations. This paper presents the testing of an automotive torque converter turbine with varying support mechanisms, transducers, excitation devices, and finally the medium into which it is submerged. As during the operational state of a torque converter turbine, it is submerged into automotive transmission fluid and the results compared to those obtained in air.

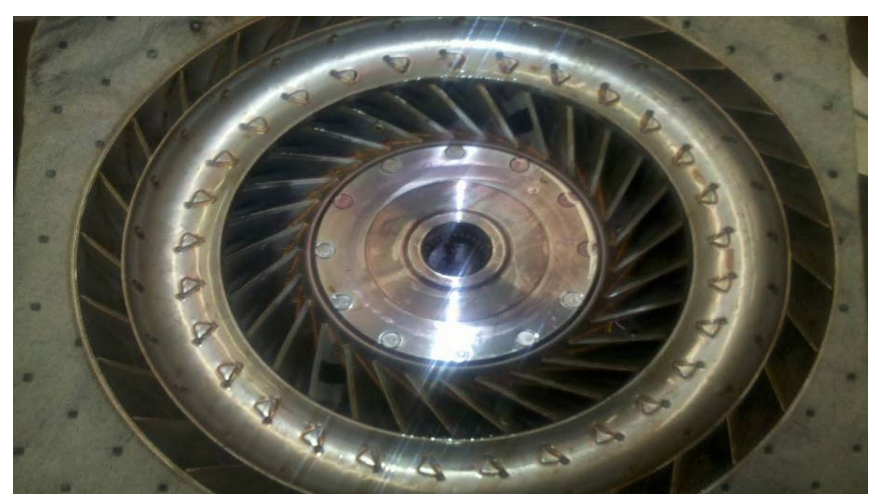

Figure 3.1: Close up image of turbine used for testing.

\subsection{Impact Jigs}

One of the most difficult aspects of testing small complex structures, such as the trailing edge blades on the torque converter turbine is being consistent in the location of force input. It is difficult to try to affix a force transducer onto the blade in this case due to a complex geometry and space issues. Since the blades of the turbine shown in Figure 3.1 are a poor candidate for shaker testing, impact testing is the preferred choice of force input. Impact testing on small structures can be difficult for those not well practiced in the use of a micro-impact hammer. Even then, in order to have a high degree of 
repeatability while performing impact testing, it is wise to create a jig to assist in both automating the impacts and making their amplitudes and position more repeatable.

The first jig discussed here is a very simple concept whereby a known, yet unmeasured, force created by a ball bearing impacting the structure is used as the excitation. This is a very precise method of maintaining consistency in the amplitude of the input force as well as the location of the impact. The second jig is an evolution of the first, replacing the ball bearing with a weighted rod, onto which a force transducer is affixed, thereby collecting an actual measurement of the input.

\subsection{Ball Bearing Impact}

The first jig takes advantage of Newton's Second Law to calculate the force input into the structure in lieu of a force transducer. The excitation is applied by means of using ball bearings of a known mass, and dropping them down a guide tube onto the blade so that they impact the same point on the structure with each drop. This method has shown to have a high degree of repeatability. The amount of energy to be put into the structure can be adjusted via the mass of the ball bearings or the height from which they are dropped.

Equation 1 is Newton's Law modified for dropping the ball bearings onto any structure. The mass of the ball bearing is $m_{b b}, g$ is the acceleration due to gravity, and $\theta_{\text {vert }}$ is the angle the surface of the structure makes with vertical.

$$
\mathrm{F}_{\text {impact }}=\mathrm{m}_{\mathrm{bb}} * \mathrm{~g} * \sin \left(\theta_{\text {vert }}\right)
$$

This equation gives a force which is used to create an artificial impact time history using a unit impulse function. The impulse is multiplied by the magnitude of the force and the autopower calculated. This autopower spectrum, seen in Figure 3.5 below, is then used to normalize each average of the autopower of the response, which in this case was measured by a laser vibrometer.

The ball bearing jig itself, shown in Figure 3.2, is simply a fixed tube aimed at the structure through which a ball bearing is dropped. Rough aiming is done by sighting the point of impact on the structure through the guide tube, small changes can be made 
afterward by several test drops to check positioning. It is very important that this jig is very sturdy so that the point of impact does not vary. Also the tube to be used should be very smooth on the inside, as a rough surface will make the ball bearing path more erratic. It is also required that the path of the ball bearing allows for it to impact the structure only once as with this method it is more difficult to determine whether a double hit occurred with the lack of a measured time history or autopower of the excitation. When using this jig in concert with a laser vibrometer, it must also be set up to make sure that the ball bearing does not cross the beam and interrupt the measurement. Alternately, when using this jig with accelerometers, it is inadvisable to impact the accelerometer directly, as this is not a true means of exciting the structure, and may cause the transducer to overload due to the direct impact.

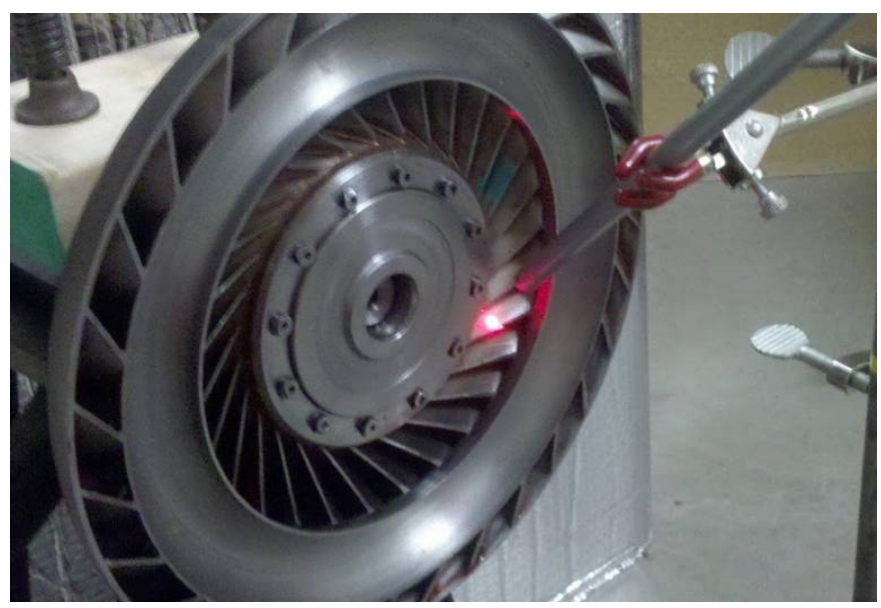

Figure 3.2: Image of the ball bearing drop jig. The red dot is the laser vibrometer reflecting off of the trailing edge blade of the turbine. The guide tube comes down from the upper right portion of the picture to guide the ball bearing to impact near the laser dot.

The ball bearing method isn't a perfect means by which to excite a structure, but it is a very good means by which to have a high degree of repeatability with your excitation on very small structures. The greatest drawback to this method is that the excitation point on the structure must allow for the ball bearing to drop away from the structure so that it does not create a double hit. As a result, it is almost impossible to use an input point that is on the top of a structure. Also, when using this method, it is impossible to collect true phase data. The exact moment at which the ball impacts the structure is unknown, and 
cannot be used as a phase reference. This also gives somewhat of an uncertain result with regards to the magnitude of the input as it is based on an idealized model. This effect is seen in Figure 3.4 when compared with the next method of excitation, in which the input force is measured instead of estimated.

\subsection{Impact Rod}

A second excitation method that was developed for this structure is an impact rod. This jig evolved from the previous in the use of a guide tube to accurately direct the input force. Not quite as compact or simple as the ball bearing method described above, this method has the advantage of being able to use an actual force transducer to measure the force imparted on the structure. This assembly also allows for very good repeatability in reference to the point of impact. Any discrepancy with regards to the energy input to the structure is alleviated because the input force is directly measured by the force transducer.

A stinger with a weight threaded on one end was slid first through a spring from a retracTable 3.ball point pen and then a steel tube. The end opposite the spring on the stinger is outfitted with a two part chuck assembly. An impact cap is threaded into the base of the chuck to create a smooth surface for the head from a micro-impact hammer to be glued securely. The entire striker assembly is shown in Figure 3.3. The guide tube of this assembly is clamped into a fixture similar to the tube into which the ball bearing assembly was clamped.

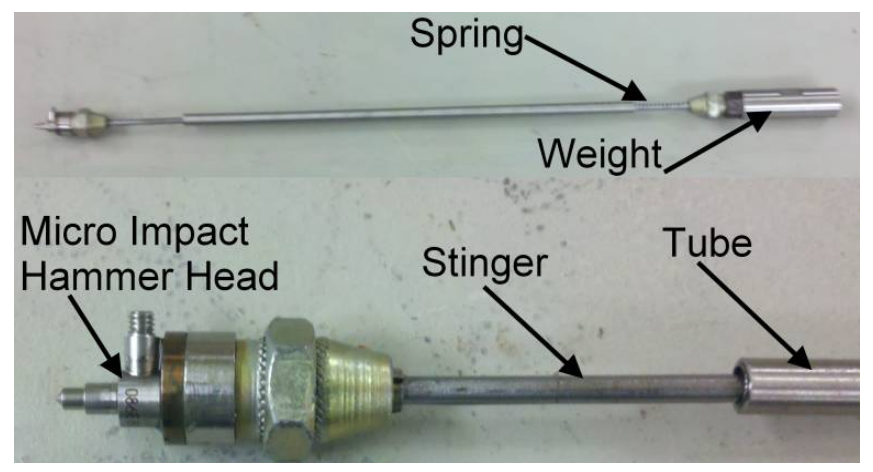

Figure 3.3: Photo of impact rod assembly depicting the various parts used. 
After an impact, the spring draws the rod assembly back to prevent it from impacting again. Double-hits are avoided easily by adjusting the initial distance of the tip from the impact point on the structure. As with the ball bearing method, the support structure should be very sturdy to prevent a loss of alignment over the various impacts. One of the main advantages of this method over the ball bearing method is that the rebound is controlled by the spring, not gravity. This means that the impact rod can be used on the structure at various angles and even on the top of the structure without worry of a second impact. The greatest disadvantage of this jig is the required clearance required to set it up. The length of the rod can be shortened, however it still will not allow it to be used in very cramped spaces.

\subsection{Comparison of Input Methods}

Figure 3.4 shows how the response of the structure is slightly different depending on the excitation method used. The FRFs from both methods in general agree quite well. The frequencies all line up, and even the amplitude at higher frequencies is very close. The difference in amplitude at the lower frequencies could be attributed to the fact that the impulse used to characterize the impact of the ball bearing was an ideal impulse, which is the amplitude of the force of the ball bearing on a perfectly rigid structure at one sample point in a time history. This would translate in the FFT as a perfectly flat response across all frequencies. An actual impact on the structure varies from this ideal model.

Figure 3.5 shows the comparison of the normalized autopower spectra between the impact methods. The time histories of both input methods were normalized by their maximum values as the amount of force in each impact were different. The larger amplitude in the measured autopower at lower frequencies explains how the FRF response for that input method might be smaller at those frequencies.

From these plots it can be stated that both methods are successful at exciting the structure to measure an FRF. In the event that a transducer to properly measure the input is unavailable, or that the structure in question is very difficult to impact with a high degree of repeatability due to small size, it is possible to collect FRF measurements using the 
ball bearing method. As stated earlier, this method sacrifices the phase data, and must be setup properly in order to maintain accuracy of input location.

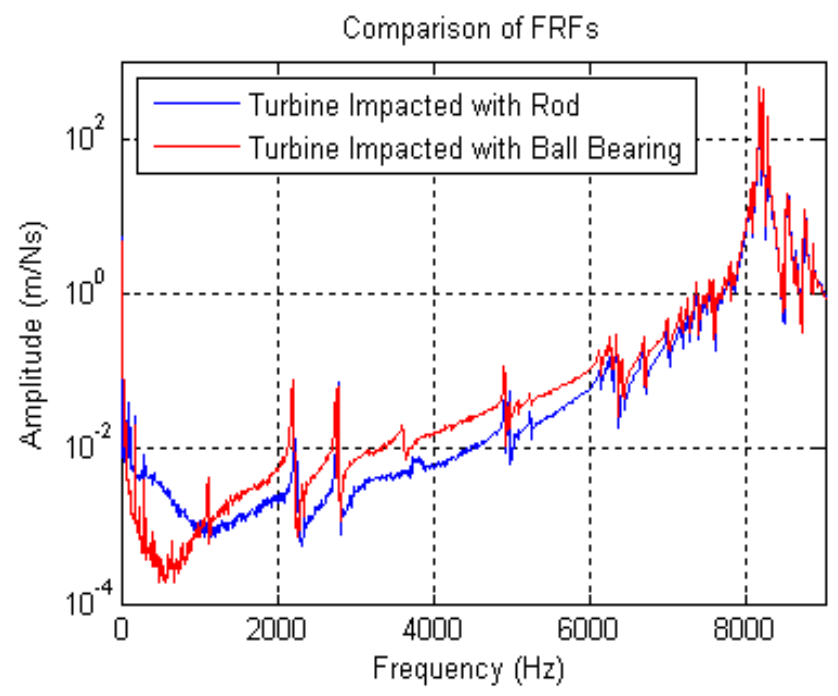

Figure 3.4: Plot comparing FRFs taken using a ball bearing as the excitation and the impact rod.

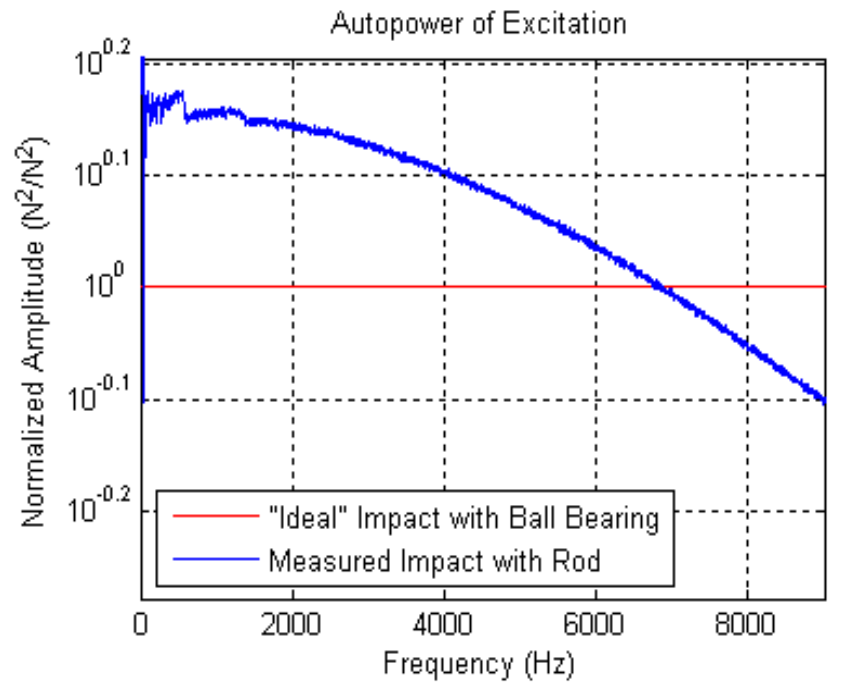

Figure 3.5: Plot comparing the autopower spectra of the "Ideal" impact with the ball bearings with the measured impact with the impact rod.

\subsection{Submerged Modal Testing}

Several difficulties occur when trying to measure the dynamics of a structure when it is in a fluid other than air. The laser vibrometer used in the early studies is an impractical tool 
with which to measure the response as any change in medium will bend the beam of the laser. Furthermore, it is very difficult to excite the structure without exciting the fluid as well, thereby changing the air fluid boundary resulting in a change in the position of the beam. Ultimately, trying to use a laser vibrometer to measure the response of a structure in something other than air results in measuring the response of the surface of the fluid the structure is submerged in.

For this reason accelerometers should be used when comparing a measurement in air with those in a liquid. Several precautions need to be taken. First, be sure that the accelerometer used is not creating a large amount of mass loading on the structure. For these studies the accelerometer used is 0.3 grams, which much is less than $0.1 \%$ of the mass of the turbine. Also, the accelerometer, along with any other transducer you want to submerge, should be hermetically sealed and have an integral cable so as to not damage the sensing elements. This is especially important in liquids that have a high electrical conductivity, such as city water, salt water, and alcohols. Oils, such as the ATF being used for these experiments, tend to have a very low electrical conductivity, and are fairly safe to submerge transducers in for short periods. For these experiments the impact rod was used and therefore the micro-impact head cable connection was wrapped in heat shrink tubing to help prevent any fluid leakage during the testing.

Another important thing to note is how the structure is coupling with the fluid. In a complex part, like this turbine, it is important to make sure that no air bubbles are trapped under/in the structure. Any pockets of air are points which are not properly coupling with the fluid, and therefore may create errors in the measurements. To be sure that the structure is in complete contact with the fluid, make sure to submerge it completely and then flip it several times within the fluid, making sure that all air passages are free from air pockets. As some structures had hollow passages that may not allow the trapped air to leak out quickly, it is advisable to let the structure "soak" in the fluid so that any air pockets have time to escape completely.

A full modal test was performed in air, with points all over the structure using a roving impact method. The same turbine was tested completely submerged in ATF. When the 
structure was submerged, it was difficult to measure at all of the points measured during the air experiment. However, all of the driving point measurements, along with several other points of interest were collected. Two points of interest on the structure were a driving point on the shell and one on a trailing edge blade. The comparison of these driving points in air and submerged is shown in Figure 3.6 and Figure 3.7.

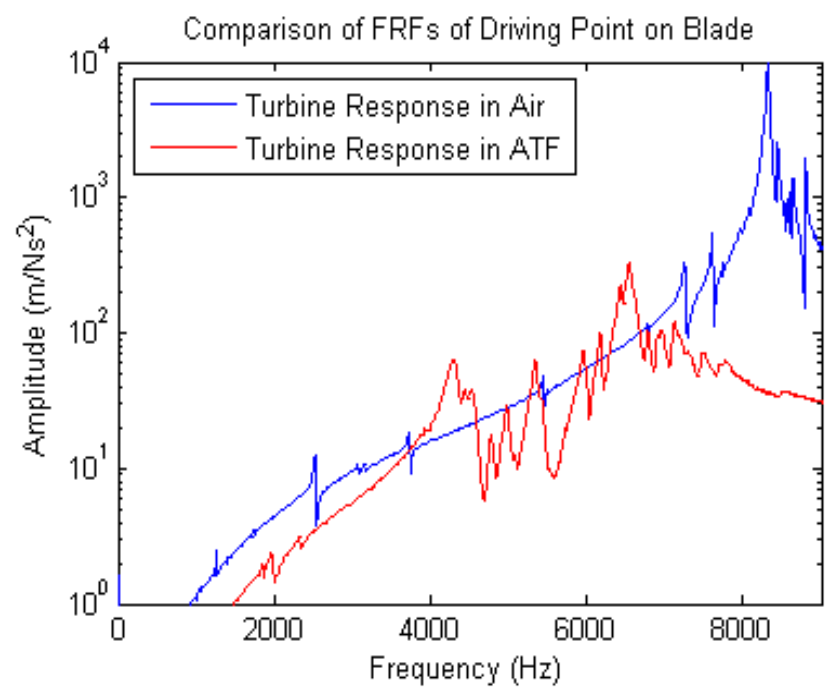

\section{Figure 3.6: FRF plot of driving point on a trailing edge blade.}

Looking at the turbine in air (dry) in Figure 3.6, it can be assumed that the main peak at around $8 \mathrm{kHz}$ is the natural frequency of the blade used for the driving point. This frequency has the largest response due to the fact that all of the blades are almost identical in shape, size, and how they are attached to the turbine as a whole, and therefore each blade exhibits around the same natural frequency. It is interesting to note that the turbine submerged in the ATF (wet) does not show this predominant peak. This might be explained by the fact that each turbine blade couples with the fluid a little bit differently, in effect smearing the natural frequencies when comparing the dry turbine to the wet turbine. 


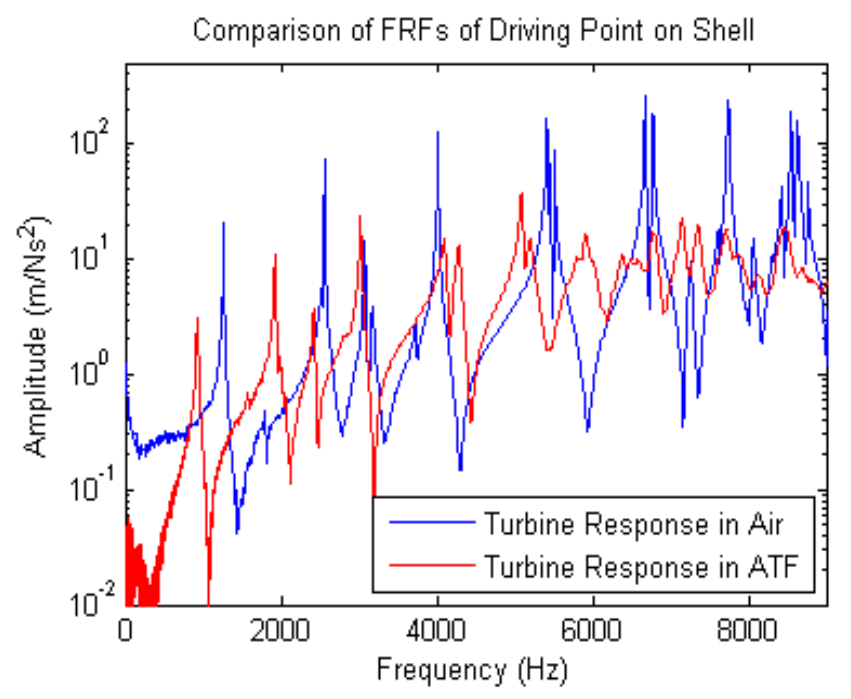

Figure 3.7: FRF plot of driving point on edge of shell.

Figure 3.7 shows that nearly all of the natural frequencies in the dry turbine can be identified in the wet turbine. It appears as if each natural frequency of this part of the structure couples with the ATF in a similar manner. In fact, upon further inspection, there exists a relationship between each of the first predominant modes that appear in this FRF. This relationship is shown in Table 3.1 .

Since each mode appears to shift by approximately the same percent, which can be correlated to an increase in mass across the entire FRF. Knowing that the natural frequency is proportional to the inverse of the square root of mass, the increase of mass across the structure can be calculated to be about $72.6 \%$. Furthermore, this can be removed from the wet turbine driving point FRF on the shell using a very simplified mass modification. Simply put, the frequencies of the FRF of the wet turbine were shifted to remove the additional mass. As an FRF is a function of frequency, the "Mass Modified" frequency is calculated by Equation 2.

$$
\mathrm{f}_{\mathrm{mm}}=\mathrm{f}_{0} *\left(1+\mathrm{C}_{\mathrm{m}}\right)
$$


Table 3.1: This Table 3.is a comparison of the first six natural frequencies that appear in the FRFs of the driving point on the shell of the turbine.

\begin{tabular}{|c|c|c|}
\hline $\begin{array}{ll}\begin{array}{l}\text { Dry } \\
(\mathrm{Hz})\end{array} & \mathrm{F}_{\mathrm{n}} \\
\end{array}$ & $\begin{array}{ll}\text { Wet } & F_{n} \\
(H z) & \\
\end{array}$ & Change \\
\hline 2560 & 1921 & $-25.0 \%$ \\
\hline 4010 & 3020 & $-24.7 \%$ \\
\hline 5410 & 4095 & $-24.3 \%$ \\
\hline 5510 & 4286 & $-22.2 \%$ \\
\hline 6670 & 5081 & $-23.8 \%$ \\
\hline 6770 & 5195 & $-23 . \%$ \\
\hline & Mean & $-23.9 \%$ \\
\hline & St Dev. & $1.0 \%$ \\
\hline
\end{tabular}

In this equation, $\mathrm{f}_{\mathrm{mm}}$ is the modified frequency vector to be used by the FRF function, fo is the original frequency vector, and $\mathrm{C}_{\mathrm{m}}$ is the mean value of the change from Table 3.1. The "Mass Modified" wet turbine FRF is shown with the dry turbine FRF in Figure 3.8.

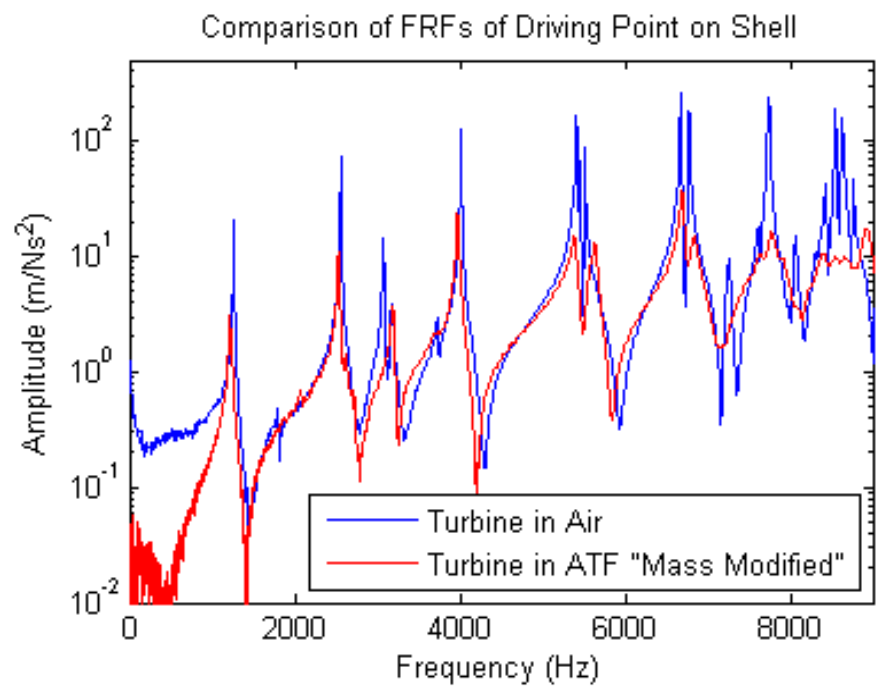

Figure 3.8: Plot of FRFs of the dry turbine and the wet turbine. The wet turbine has had its frequency scaled in an attempt to remove the additional mass coupling caused by the fluid.

The FRFs of the dry turbine and the wet turbine at this driving point match up very well after the modification. The amplitude of the peaks is not quite the same in both FRFs, and 
some of the modes have disappeared, but it is hypothesized that this can be accounted for by the additional damping caused by the coupling of the fluid with the structure, or that those modes are coupling with the fluid differently than the primary modes shown here.

One of the causes for the difference in the amount of mass coupling in this structure depending on the measurement location may have to do with the amount of surface area that is in contact with the fluid with relation to the motion of the structure. Since the shell is a large free surface area, the coupling of mass with that part of the structure is very uniform and easy to predict. The blades, however, have a much smaller surface area, along with more constrained boundary conditions which make the amount of surface area in motion on that part of the structure more difficult to predict. It could be assumed that the effective mass modification is then some function of the density of the liquid into which the structure is submerged and some surface integral of the deformation of the body

\subsection{Conclusions}

Two methods of force input were presented. The ball bearing jig is very good for high repeatability in quick tests on small structures. The major drawback of this test method lies in the loss of phase data, and the limitation on the input points possible for testing structures with this method. The ball bearing jig evolved into the impact rod jig, which also has very good repeatability as well as accuracy of measurement. The impact rod also has the advantage that it can be used on any part of a structure without worry of the clearance needed for a ball bearing to avoid a double hit. The main disadvantage of the impact rod is that it requires a lot of room for setup, and may not be feasible in tight spaces.

Taking data on a structure that is fully submerged in a fluid has several challenges. Precautions must be taken to ensure that the transducers used to make measurements are working properly. The structure must be completely submerged in the fluid and free of any air pockets that may cause errors in the measurements due to incorrect coupling of the structure with the fluid. 
After reviewing the data comparing the dry turbine to the wet turbine, several important points can be made. Some parts of the turbine, like the shell, behave in a manner that could easily be predicted when submerged in ATF due to a large surface area that is allowed to move freely. The behavior of the blades seems to be a little more erratic when comparing the wet turbine to the dry turbine FRF with regards to a more fixed point on the structure, such as the turbine blades. As the blades are smaller and far more constrained than the shell of the turbine, there is less effective mass added to that portion of the structure. As a result, the effective mass coupling added by submersion into a fluid is dependent on the amount of surface area of the structure that is both in motion and in contact with the fluid.

\subsection{References}

(1) Cakar O, Sanliturk KY. Elimination of Transducer Mass Loading Effects from Frequency Response Functions. Science Direct Mechanical Systems and Signal Processing. 2005; Volume 19: pp. 87-104.

(2) Heylen W, Lammens S, Sas P. Excitation Considerations. Modal Analysis Theory and Testing, Katholieke Universiteit Leuven. 1997.

(3) Homm, Ehrlich J, Peine H, Wiesner H. Experimental and Numerical Investigation of a Complex Submerged Structure, Part I: Modal Analysis. Acta Acustica united with Acustica. 2003; Volume 89: pp. 61-70. 


\section{Chapter 4 - Characterizing Torque Converter Turbine Noise ${ }^{1}$}

C. Walber ${ }^{1}$, J. Blough ${ }^{2}$, C. Anderson ${ }^{2}$, M. Johnson ${ }^{2}$, J, Schweitzer ${ }^{3}$

${ }^{1}$ PCB Piezotronics

3425 Walden Ave Depew, New York, USA

${ }^{2}$ Michigan Technological University, Department of Mechanical Engineering

1400 Townsend Dr. Houghton, Michigan, USA

${ }^{3}$ General Motors Powertrain

Pontiac, Michigan, USA

\subsection{Abstract}

As noise generated by other automotive components decreases, the noise generated by a more compact torque converter has the potential to become more evident. It is therefore important to characterize and understand any noise phenomena generated by the torque converter. The research discussed in this paper concerns a specific noise event within the torque converter that will be referred to as "turbine noise". In some torque converters operating at a high input torque, a high amplitude narrowband noise between 4 and $6 \mathrm{kHz}$ has been observed. The turbine noise appears to go away with the addition of notches to the trailing edges of the turbine blades. It has been hypothesized that the turbine noise is caused by Von Karman Vortex Shedding. The goal of this research was to determine the source of the noise, to characterize the flow that set up the noise condition, and to determine why trailing edge notches eliminate or reduce the noise. Several modifications to the torque converter turbine were made to assess their effects on turbine noise. Acoustic measurements were taken along with internal measurements of blade surface acceleration, pressure and strain acquired through use of microwave telemetry. Analysis

\footnotetext{
${ }^{1}$ The material contained in this chapter has been submitted to the Society for Experimental Mechanics Journal: Experimental Techniques
} 
of the test results confirmed that the characteristics of turbine noise follow the conditions for Von Karman Vortex Shedding.

\subsection{Introduction}

Torque Converter Operation - The automotive torque converter is the powertrain component that multiplies engine torque to help launch the vehicle from a stop. It is a hydrodynamic device which connects the engine to the transmission gears. As such it absorbs torsional torque disturbances from the engine and allows the engine to idle without requiring a separate mechanical clutch. Modern automotive torque converters are made up of three elements, a mixed flow pump and turbine, and an axial flow stator. Figure 4.1 is a basic schematic of a torque converter, including arrows showing the direction of the toroidal flow induced during operation. Maximum efficiency is achieved during high speed ratio operation while maximum torque multiplication is attained at turbine speed equal to zero, commonly referred to as stall. Speed ratio (SR) is defined as the ratio of the turbine speed to pump speed.

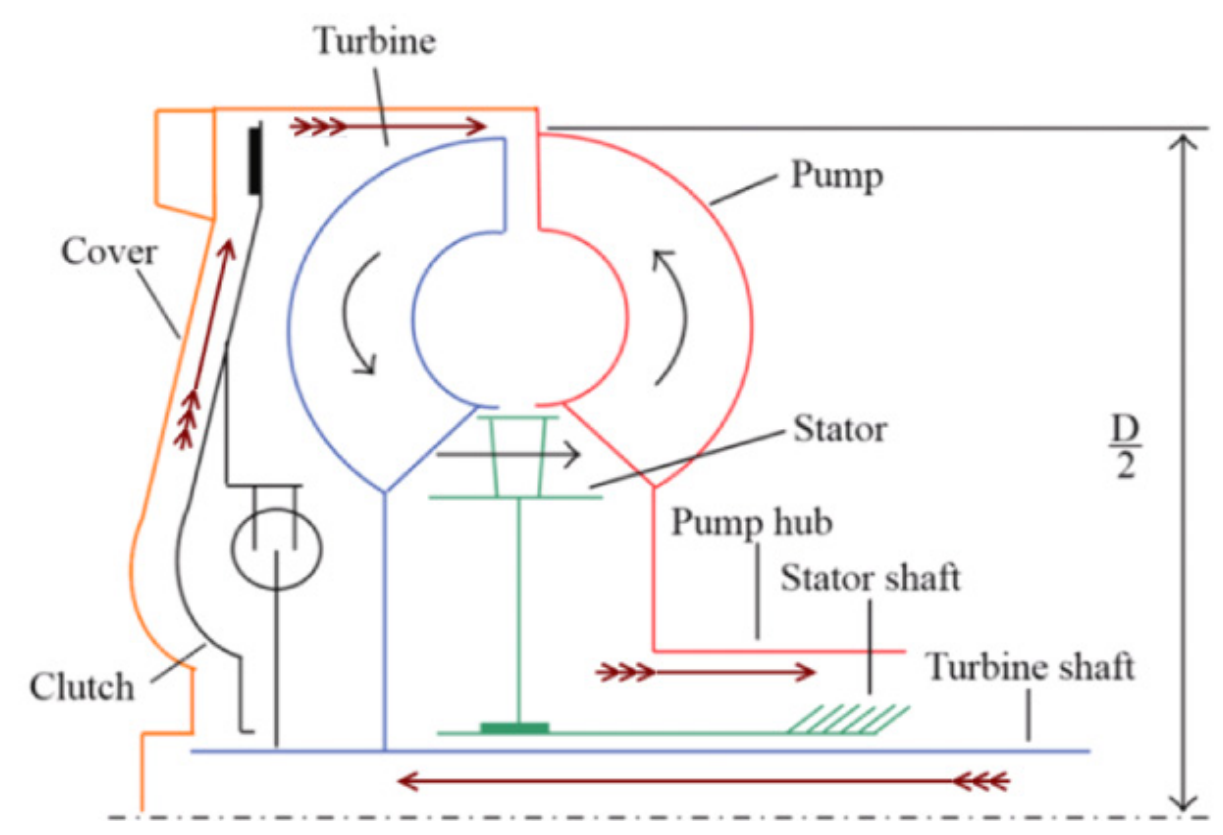

Figure 4.1: Cross section of an automotive torque converter; toroidal flow is indicated by the direction of the arrows with no tail. Arrows with tails indicate the normal cooling flow (1) 
In the basic operation of a torque converter, the pump is driven by the engine through connection of the engine crankshaft and flexplate to the cover of the torque converter. Rotation of the pump imparts a centrifugal force on the automatic transmission fluid (ATF). As depicted in Figure 4.1, this causes the fluid to flow from the inner radius of the pump flow path to the outer radius where it is directed into the turbine. The turbine turns the flow and directs it radially inward to the stator which guides the flow back into the pump in a favorable angle with regards to pump operation. The flow through a bladed element causes a change in the angular momentum of the fluid across the element which results in a torque being applied to the shaft attached to that element. The turbine is attached to the turbine shaft which is the input to the transmission gears. The stator shaft is grounded through a one-way clutch to the transmission case, and stator torque is added to pump torque. Summing the element torques through a free body analysis results in the torque balance shown in Equation 1. Thus, the turbine torque, which is the output, is greater than the pump torque, which is the input, and torque multiplication occurs.

$$
T_{\text {turbine }}=T_{\text {pump }}+T_{\text {stator }}
$$

When the torque converter is operating at high speed ratios, a clutch can be engaged to create a direct shaft connection between the engine and transmission to bypass the hydrodynamic inefficiency of the torque converter.

\subsection{Turbine Noise}

Torque Converter operational and performance parameters are generally viewed relative to the speed ratio. For the particular torque converter geometry studied, a high amplitude narrowband noise between 4 and $6 \mathrm{kHz}$ occurs at operation with input torque above 300 $\mathrm{Nm}$ and speed ratio near 0.5 . Other converters displayed a similar phenomenon at higher torques and different frequencies. This turbine noise was detectable in the interior of some early non-production development vehicles. After some research it was determined that notching the trailing edge of the turbine blades reduced the noise to a level that could not be detected. The noise is thought to be a result of a self-excited vibration phenomenon known as Von Karman Vortex Shedding. 
Some turbines are more susceptible to the noise phenomenon than others, possibly due to variability in structural damping and acoustic transmissibility of the torque converter on the whole. The sensitivity could be due to a combination of variability in turbine braze quality, hub attachment, and blade trailing edge geometry. In addition, prior testing had demonstrated that machined from solid turbines, and unbrazed turbines do not exhibit this noise behavior. It was hypothesized that the turbine variations listed, along with adding notches to blade trailing edges, alter either the structure of the turbine or the flow exiting the turbine sufficiently to reduce or eliminate the noise phenomenon. As stated previously, the root cause of the turbine noise phenomenon was thought to be Von Karmen Vortex shedding. In this type of flow, the vortices couple with a natural frequency of the turbine near the vortex frequency and cause a self-excited vibration, resulting in the turbine shell being a sounding board for the turbine noise. Sound measurements were taken of torque converters tested on a dynamometer that has a special test fixture which contains acoustic treatments. Figure 4.2 shows the results depicted in a frequency over speed ratio spectrogram for converters with turbines that contained blades without and with notches on the trailing edges.

The turbine noise, circled in white, could be heard as a rather high pitched whistle over the rest of the sound normally generated by the torque converter. The spectrograms in Figure 4.2 clearly show that the addition of a notch to the trailing edge of the turbine blades greatly diminishes the turbine noise. 

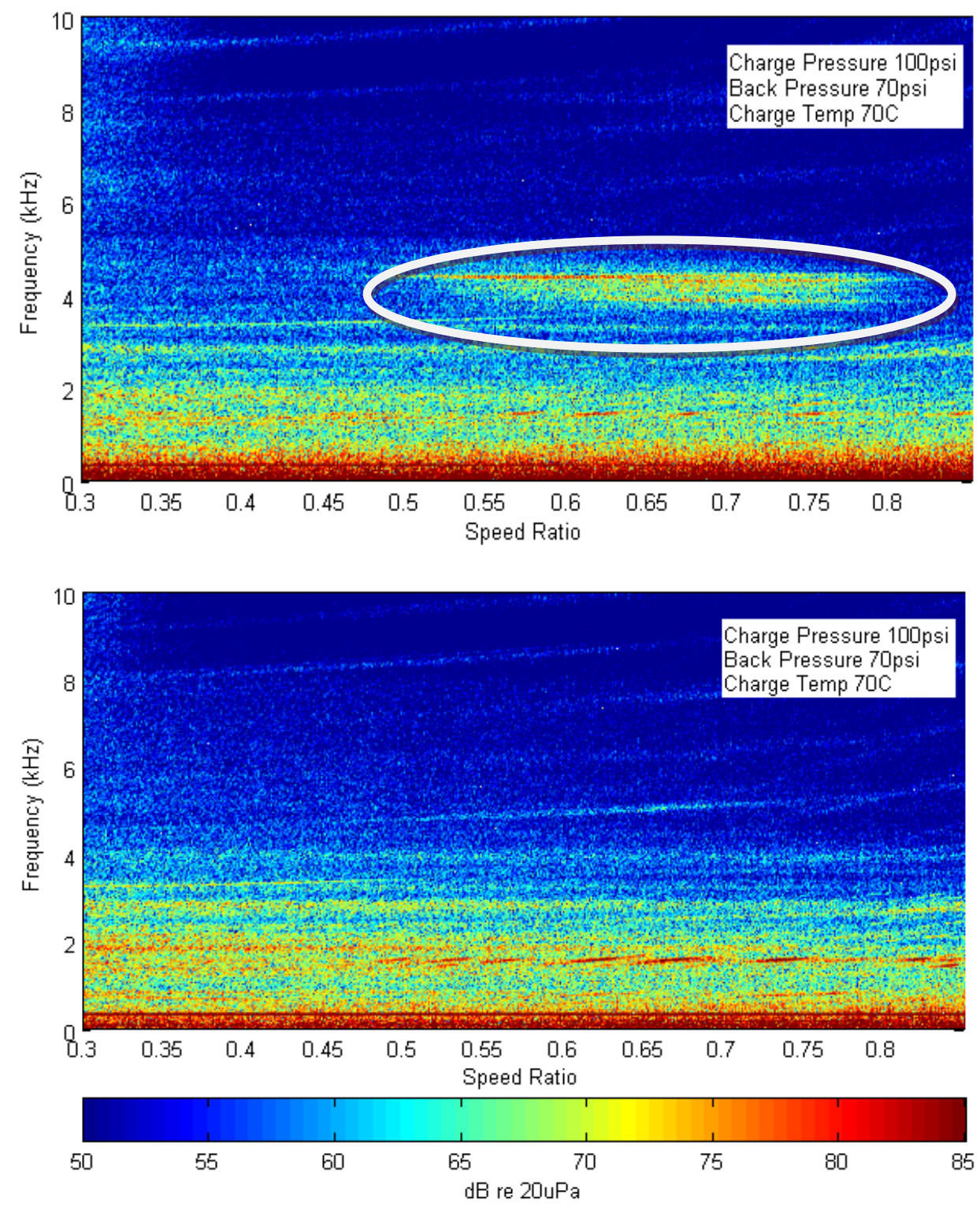

Figure 4.2: Nearfield acoustic measurement of automotive torque converter equipped with an un-notched trailing edge turbine (Top) and a notched trailing edge turbine (Bottom) during simulated vehicle launch on dynamometer with an input torque of $350 \mathrm{Nm}$. The area circled in white denotes the Turbine noise.

Since the noise phenomenon is very narrow in the frequency domain, an indicator function can be created using a bandpass filter. A sound pressure level (SPL) curve can then be calculated and used to determine the presence of turbine noise as well as any change in the noise over the course of various turbine alterations. The filter utilized is a finite impulse response (FIR) type bandpass filter with a pass band from $3.5 \mathrm{kHz}$ to 4.5 
kHz. The resulting SPL curves in Figure 4.3 were created from the data shown in Figure 4.2 .

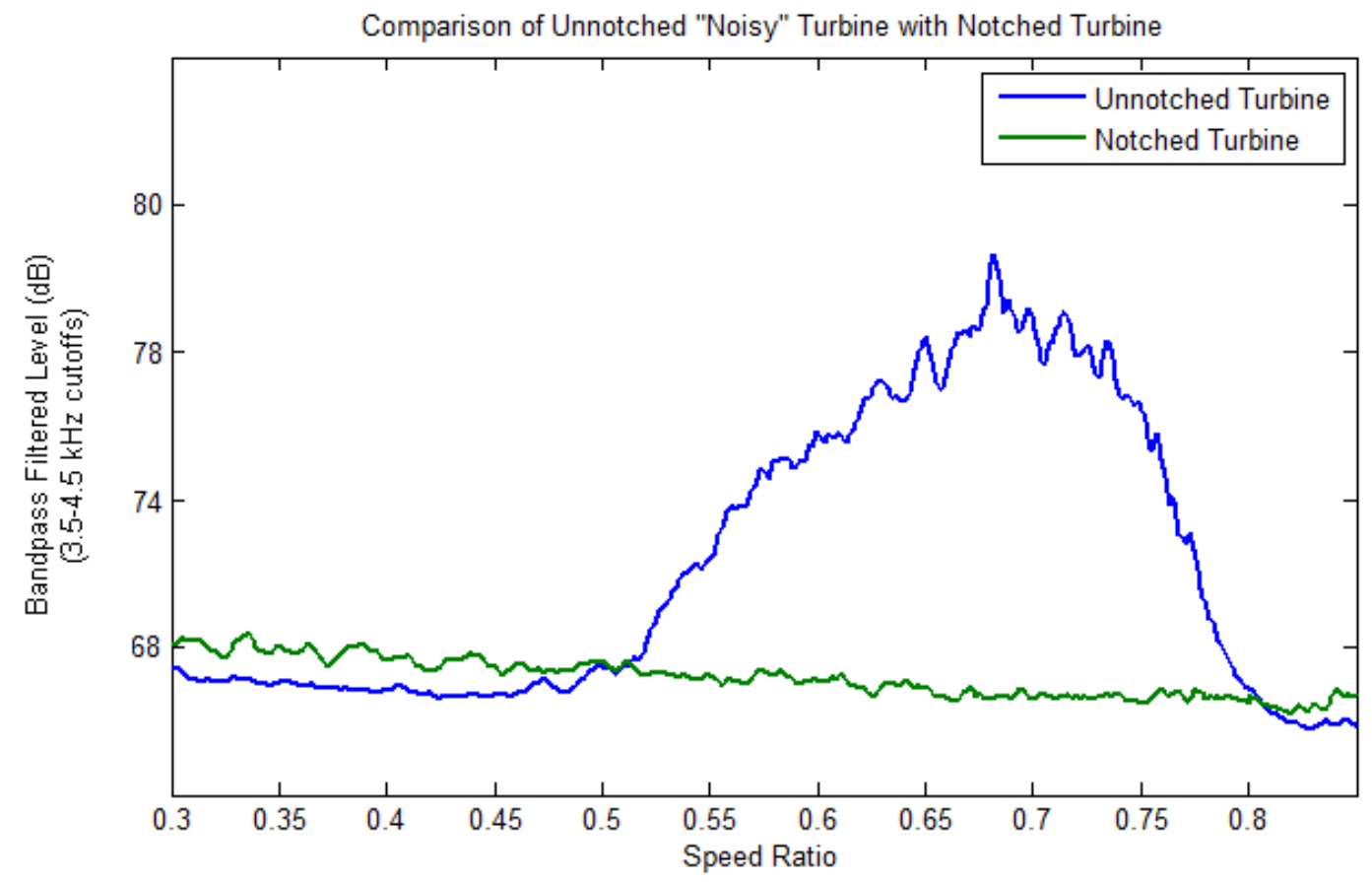

Figure 4.3: Comparison of noise from an un-notched "Noisy" turbine and a notched quiet turbine during simulated vehicle launch with an input torque of $350 \mathrm{Nm}$.

The peak SPL of the un-notched turbine is over three times the peak SPL of the notched turbine in Figure 4.3. The "noisy" range of this torque converter starts at around 0.5 SR and continues up to $0.8 \mathrm{SR}$. It should be noted that these two tests are a comparison of two different turbines. For the remaining tests, measurements were made on the same turbine for a specific test before and after modifications to eliminate effects of part to part variability. Because of this, several different turbines were used for the various tests. Thus, a baseline unaltered turbine, is included with each experimental battery as a control for that particular group.

\subsection{Vortex Shedding and Vortex-induced Vibration}

The flow phenomenon suspected of being the source of turbine noise is Von Karman vortex shedding. In Vortex shedding incidents, an unsteady flow occurs around bluff bodies with specific fluid velocities. The flow past the object creates low pressure 
vortices that alternate downstream of the object. Common structures on which vortices can form include power cables, bridges, offshore pipelines, heat exchangers, and towers. Figure 4.4 shows a side view of an airfoil in a transverse flow, with the alternating vortices of light and dark gray.

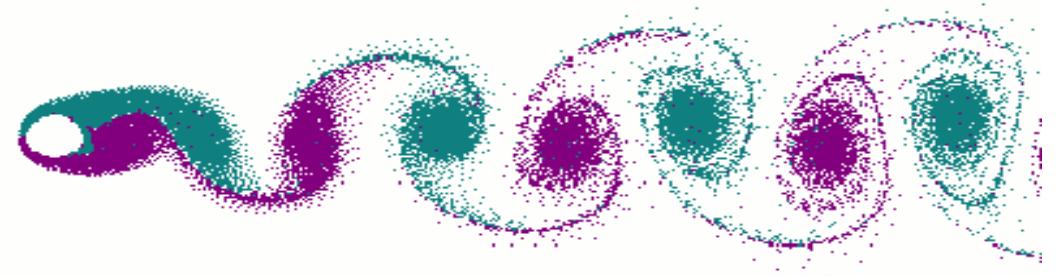

Figure 4.4: Side view of an airfoil in a transverse flow causing a Von Karman Vortex Street.(3)

Vortex shedding can occur in flows with Reynolds Numbers (Re) ranging from 47 to as high as $10^{7}$ (4). Re, a nondimensional description of velocity, is the product of free stream velocity (U in mps) and characteristic length ( $\mathrm{D}$ in meters), divided by kinematic viscosity $\left(v\right.$ in $\left.\mathrm{m}^{2} / \mathrm{s}\right)$, shown in Equation 2.

$$
R e=\frac{U D}{v}
$$

Various geometries use different characteristic lengths for calculating Re. Characteristic lengths for a sample of geometries are depicted in Figure 4.5. In this case Re is used in conjunction with the dimensionless Strouhal $(\mathrm{St})$ number to characterize oscillating flows. Strouhal Number is an experimentally determined quantity for a given geometry over a range of Re. It is generally constant over Re for a particular geometry, and typically lies in the range of .1 to .3 for oscillating flow. The equation for Strouhal Number is shown in Equation 3.

$$
S t=\frac{f D}{U}
$$

The parameters for Strouhal Number are the vortex frequency ( $\mathrm{f}$ in $\mathrm{Hz}$ ), the characteristic length of the structure ( $\mathrm{D}$, in the case of a cylinder, the diameter in meters), and the free stream velocity of the flow around the structure ( $U$ in $\mathrm{mps}$, the same as $U$ for the 
Reynolds Number). The range of Strouhal Number over Reynolds Number for various geometries is shown in Figure 4.5.

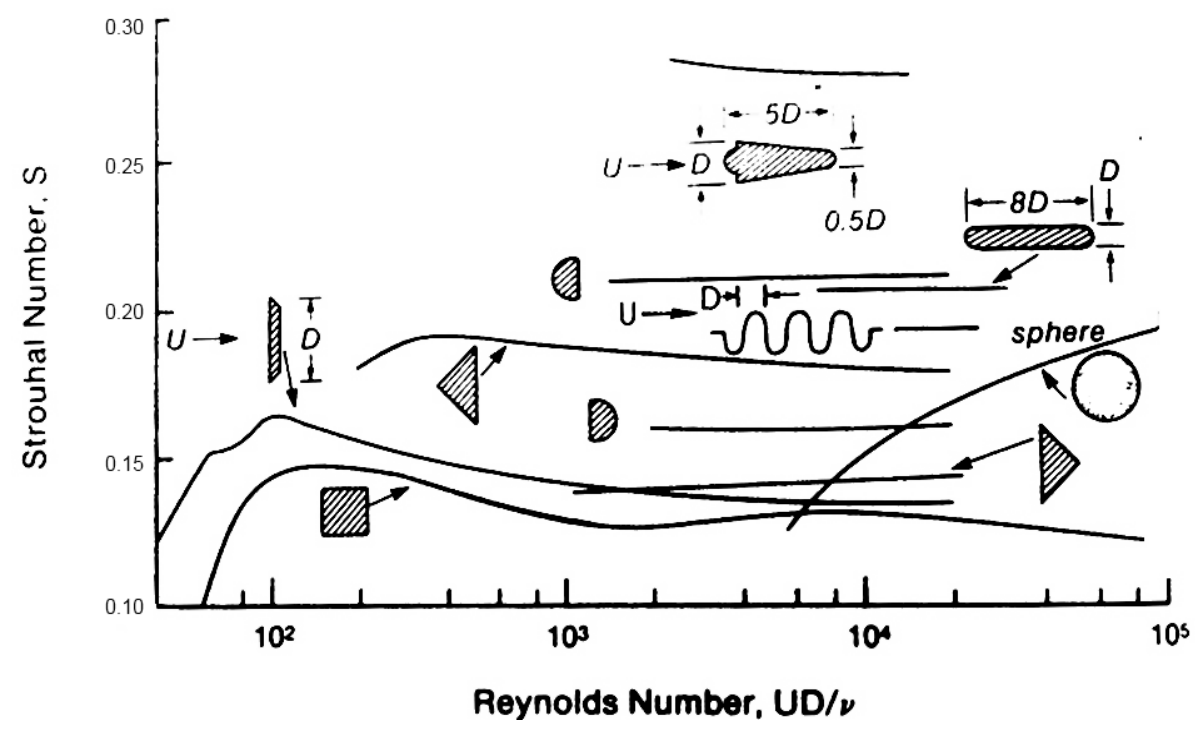

Figure 4.5: Strouhal Number for various geometries over Reynolds Number. (4)

Ultimately, the Strouhal Number is a means of determining frequency of vortex release. As each vortex sheds from the structure, the body will tend to move toward the low pressure zone left in the wake of the vortex. Therefore, if the vortex frequency matches close to a natural frequency of the structure, the structure can resonate and a self-excited vibration can occur. This is called lock-in, and while the structure resonates at its natural frequency the vortices shed at the natural frequency of the structure. The top portion of Figure 4.6 shows a plot that tracks vortex frequency as it approaches the natural frequency of the cylinder. Lock-in is the region in Figure 4.6 where the data points indicating the vortex frequency begin to follow a horizontal line at a natural frequency of the cylinder.

Once the vortex frequency is theoretically far enough away from the structure's natural frequency, the vortex frequency will part from the natural frequency as the interaction between the structure and fluid becomes too weak to continue to support the feedback required for the self-excited vibration. If no natural frequency is present, the vortex frequency will remain near the "Stationary Cylinder Shedding Frequency" line indicated in Figure 4.6. The motion of the cylinder (shown in the bottom portion of Figure 4.6) is 
driving the vortex frequency (shown in the top portion of the figure) and vice versa. The top vertical axis of Figure 4.6 is the dimensionless vortex frequency, with $\mathrm{f}$, the natural frequency of the system, normalizing $f_{s}$, the vortex frequency. The lower plot shows the amplitude of the motion of the cylinder, Ay, as it is normalized by the diameter of the cylinder, D. The horizontal axis in both cases is the free stream velocity of the flow, $U$, as it increases. $U$ is normalized by the product of the natural frequency and the diameter of the structure.

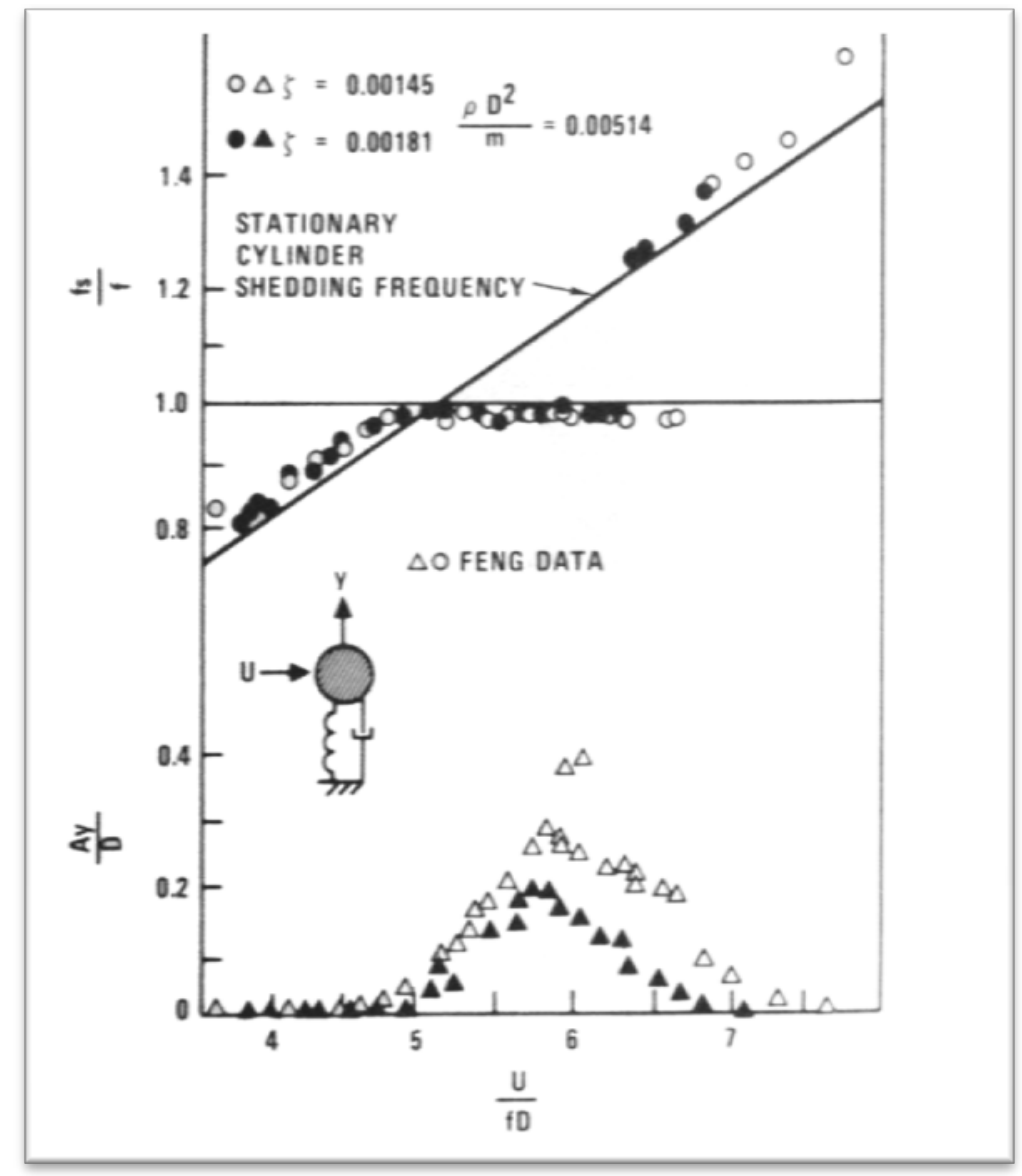

Figure 4.6: Vortex-induced vibration of a spring-supported, damped circular cylinder. $\zeta$ is the damping ratio of the structure. As seen in the two data sets, the lower the damping of the structure, the more motion caused by vortex lock-in, and the longer the vortices remain locked into the motion of the cylinder. (4)

In the case of vortex lock-in for turbine noise, the free stream velocity is the toroidal flow as it comes off of the trailing edge of the turbine blades. The characteristic length for use in the Strouhal Number is a geometric feature normal to the direction of flow, possibly 
the thickness or rake length of the blade trailing edge. Since the phenomenon is thought to be lock-in, the vortex frequency would coincide with the natural frequency of the structure for a long period. The spectrogram plots in Figure 4.2 show that this frequency is in the $4-6 \mathrm{kHz}$ range.

The most common means to reduce the occurrence of vortex-induced vibration is to add some vortex suppression device, such as helical strakes, axial slats, streamlined fairings, or spoiler plates. Blevins (4) stated that vortex-induced vibrations of a plate are suppressed by streamlining the trailing edge. Some computer fan manufacturers advertise the use of vortex control notches to reduce the noise of their fans. Figure 4.7 shows a diagram of the flow over a conventional fan trailing edge, and a trailing edge with "vortex-control" notches. (5) The manufacturer states that these notches create a disturbance upstream of where the vortices form, thereby breaking up the vortex street before it fully forms or by preventing the vortices from coupling with the structure to produce a self-excited vibration. This is similar to the turbine noise phenomenon.

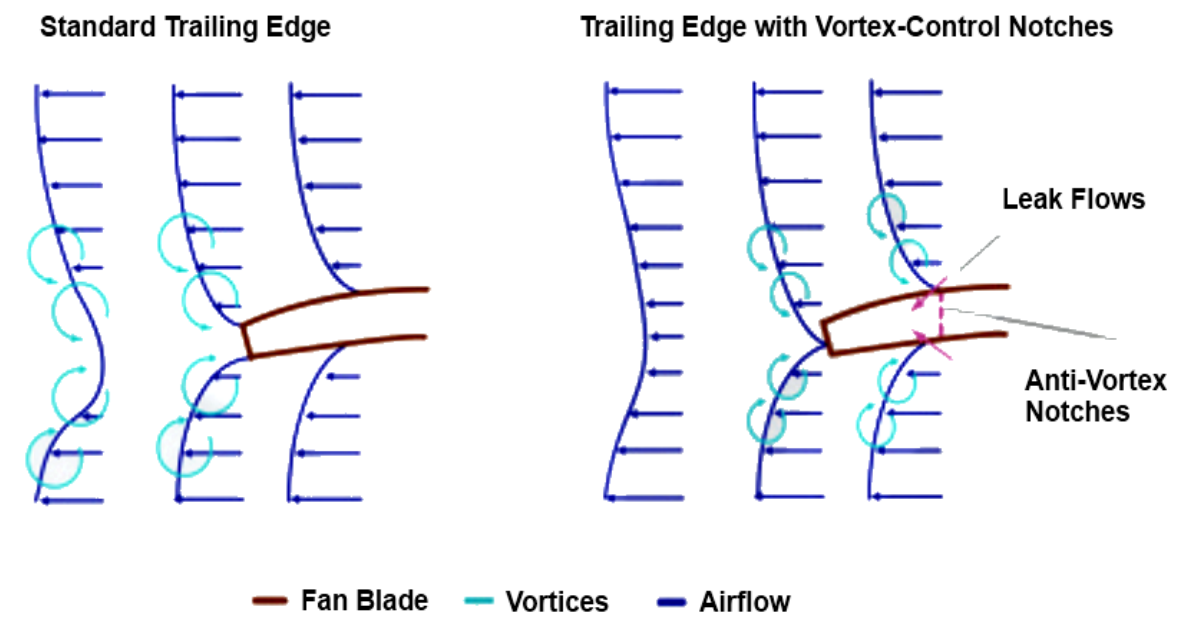

Figure 4.7: Diagram of flow over a standard fan blade trailing edge, and the trailing edge of a fan made by Noctua with their Vortex-Control Notches. (5)

\subsection{Experimentation}

In order to investigate the Vortex shedding theory as the source of the turbine noise, two types of experiments were conducted with torque converters having various blade geometries. Structural testing was performed on the turbine alone to determine whether 
or not a turbine known to generate noise had unique structural properties that could be used to identify it as a potentially noisy turbine. Testing on a dynamometer was also performed in order to better understand the noise during torque converter operation.

As previously stated, it had been established that adding a notch to the trailing edges of turbine blades reduces the turbine noise. Figure 4.8 contains pictures of the turbine blade trailing edges with and without notches. The particular un-notched turbine shown was used in later testing with the wireless telemetry, and is shown again in later images with the modifications for each test.
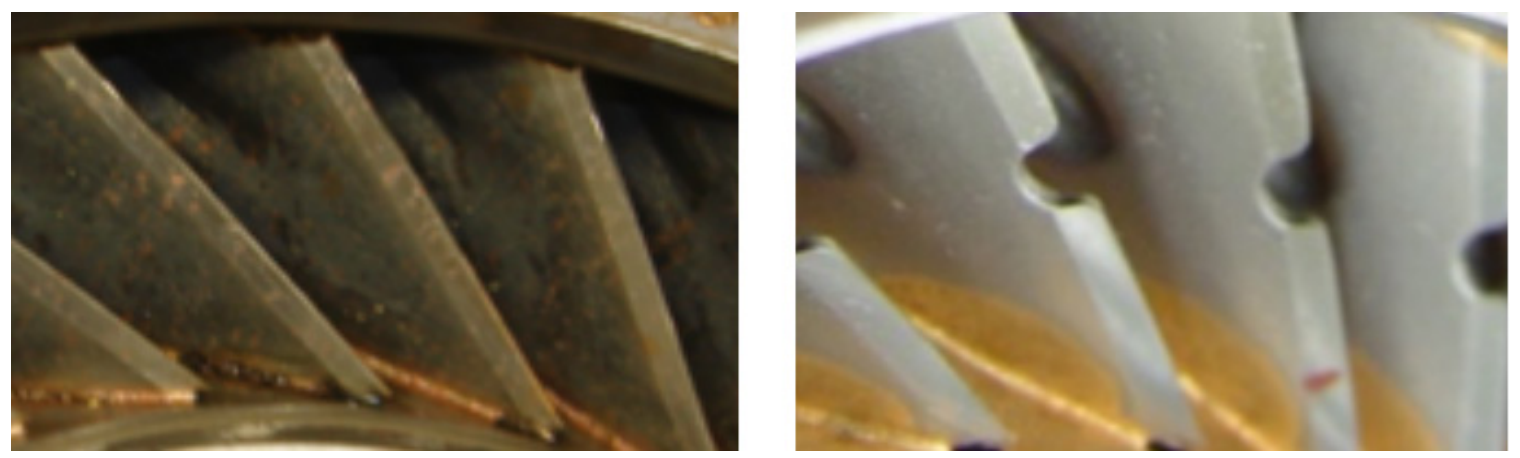

Figure 4.8: Photographs of an un-notched turbine that exhibits the "Turbine Noise" (Left) and a notched turbine (Right).

Structural Testing -Structural testing was performed on both known noisy and quiet turbines in an effort to determine if the frequency response alone can be used to predict whether or not a turbine will generate noise. Figure 4.9 shows a plot of the autopower response at the trailing edge of one blade of five different turbine constructions. The method used for excitation was a ball bearing dropped from a constant height onto the trailing edge of a turbine blade. This excitation method is further outlined by Walber (6). Since the noise is known to be affected greatly by modifications to the trailing edge, only driving point measurements were taken at the trailing edge of turbine blades.

There were some differences in the frequency response between all five of the turbines tested, however nothing definitive stood out to differentiate the two turbines that made noise from the ones that did not. The main natural frequencies of the two noisy turbines (the un-notched turbines) were separated by about $1000 \mathrm{~Hz}$, with all of the quiet turbines (the machined from solid and two notched turbines) interspaced in between. 


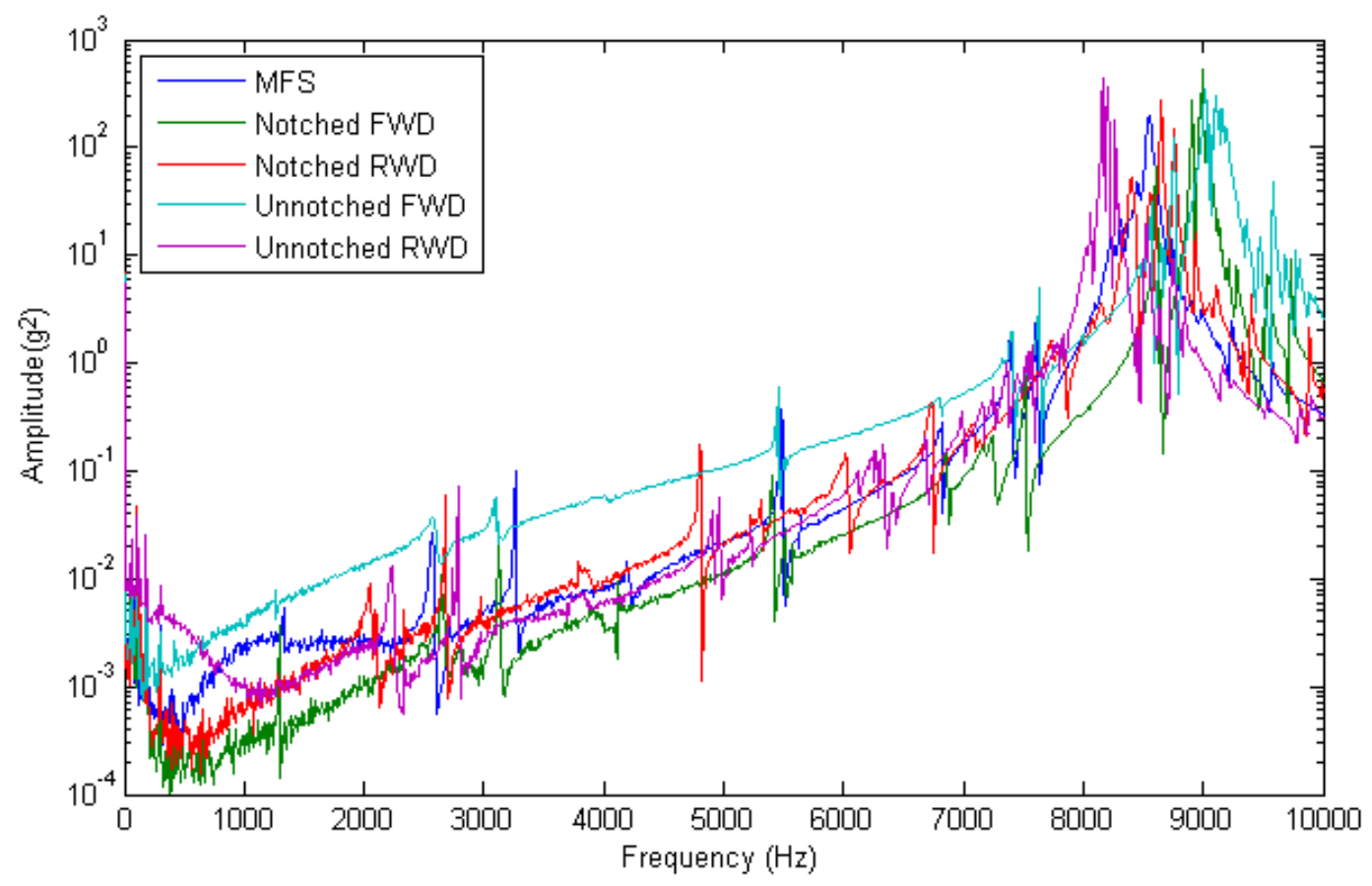

Figure 4.9: Comparison of autopower response of turbines known to be noisy during operation (Both un-notched turbines) to those that were known to be quiet during operation (MFS and both notched turbines) in air.

Modal testing was performed to determine if blade to blade variation on a specific turbine was as great as the variation from turbine to turbine. A roving impact method of excitation was used with three accelerometer references for the frequency response functions. Reference data was taken at the center of one of the turbine trailing edge blades, on the hub of the turbine, and on the outer shell of the turbine. Response measurements were collected using an impact hammer at three points along four different blades, eight points around the hub, 32 points around the surface of the shell of the turbines, and one point that corresponded to the driving point blade. Some of this data is shown in Figure 4.10 and Figure 4.11. 


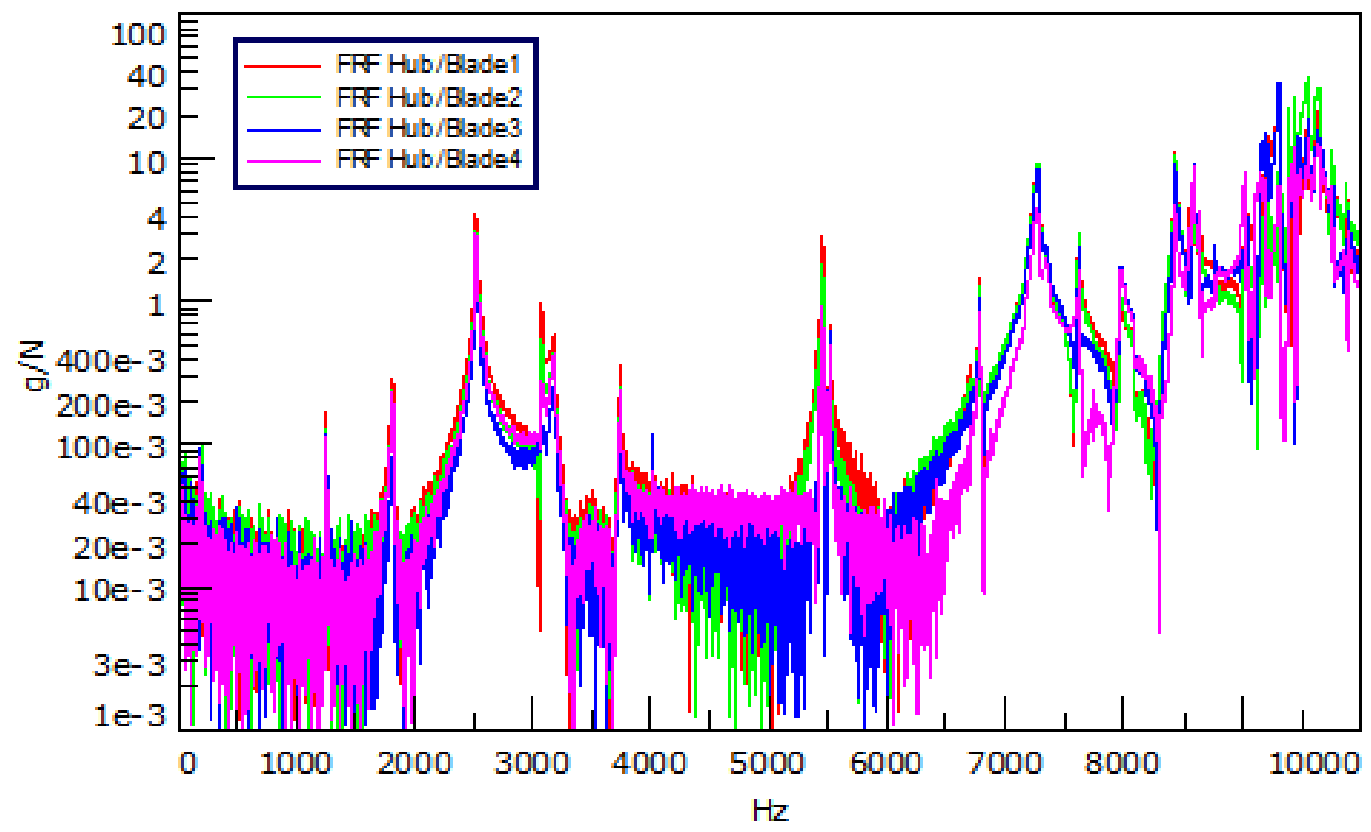

Figure 4.10: Comparison of frequency response functions of a reference point on the hub of the un-notched turbine of a noisy turbine with four trailing edge blades tested in air.

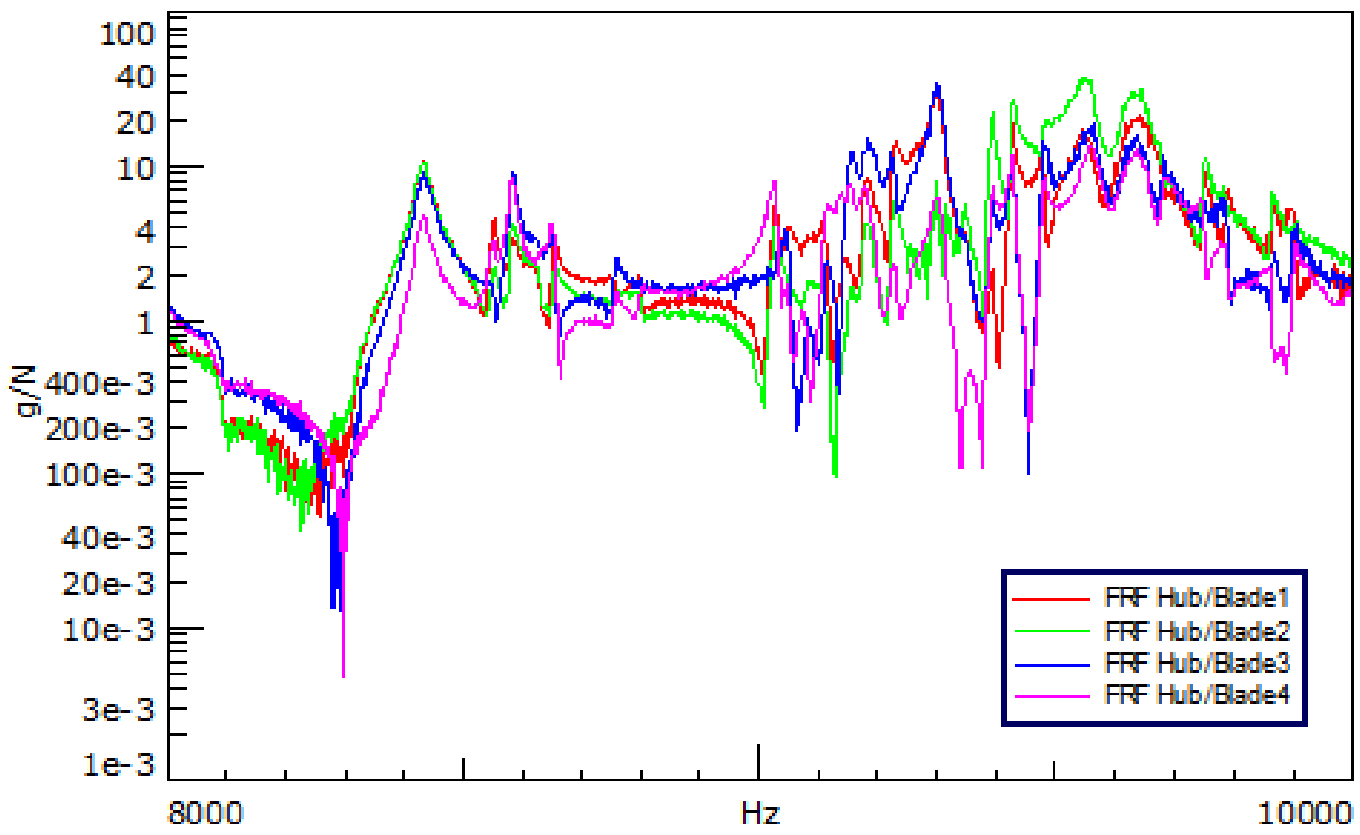

Figure 4.11: Comparison of frequency response functions of a reference point on the hub of the un-notched turbine of a noisy turbine with four trailing edge blades zoomed in to the natural frequencies thought to be associated with the trailing edge blades tested in air. 
Figure 4.10 shows that each blade has a very small variation with respect to the others when the hub is used as a frequency response reference point and looking at the entire frequency range. However, Figure 4.11 shows what is believed to be the individual natural frequencies associated with each turbine blade. It is apparent from review of the Figure 4.that it would be difficult to use variation of individual blade frequencies to determine whether or not a turbine is noisy. In addition, there does not appear to be a natural frequency at $4 \mathrm{kHz}$, the frequency at which the turbine noise occurs. However, this data was taken in air, which is not the normal operating medium for the torque converter.

The vibration mode of the turbine at the frequency of the noise in the operating medium, ATF, was investigated next. Only one turbine, an un-notched turbine, was tested both in and out of ATF since it was already determined that it could not easily be differentiated dynamically from a notched turbine or other quiet turbines. The same physical points on the structure were measured when the turbine was placed in ATF as when it was in air.

The data from the submerged testing is shown in Figure 4.12. The physical points referenced on the turbine where the data was measured are identical to those measured in Figure 4.10. The comparison between test results in air and in ATF shows that the natural frequencies of the turbine in ATF shift almost uniformly to lower frequencies, while the overall amplitude of the data remains about the same. This indicates that the addition of ATF to the turbine acts as mass loading with regards to vibration, while very little damping can be detected here. As can be seen in the peaks shown in Figure 4.12, the shift in the natural frequencies placed one resonance right at $4 \mathrm{kHz}$ and a second resonance at around $4.3 \mathrm{kHz}$. 


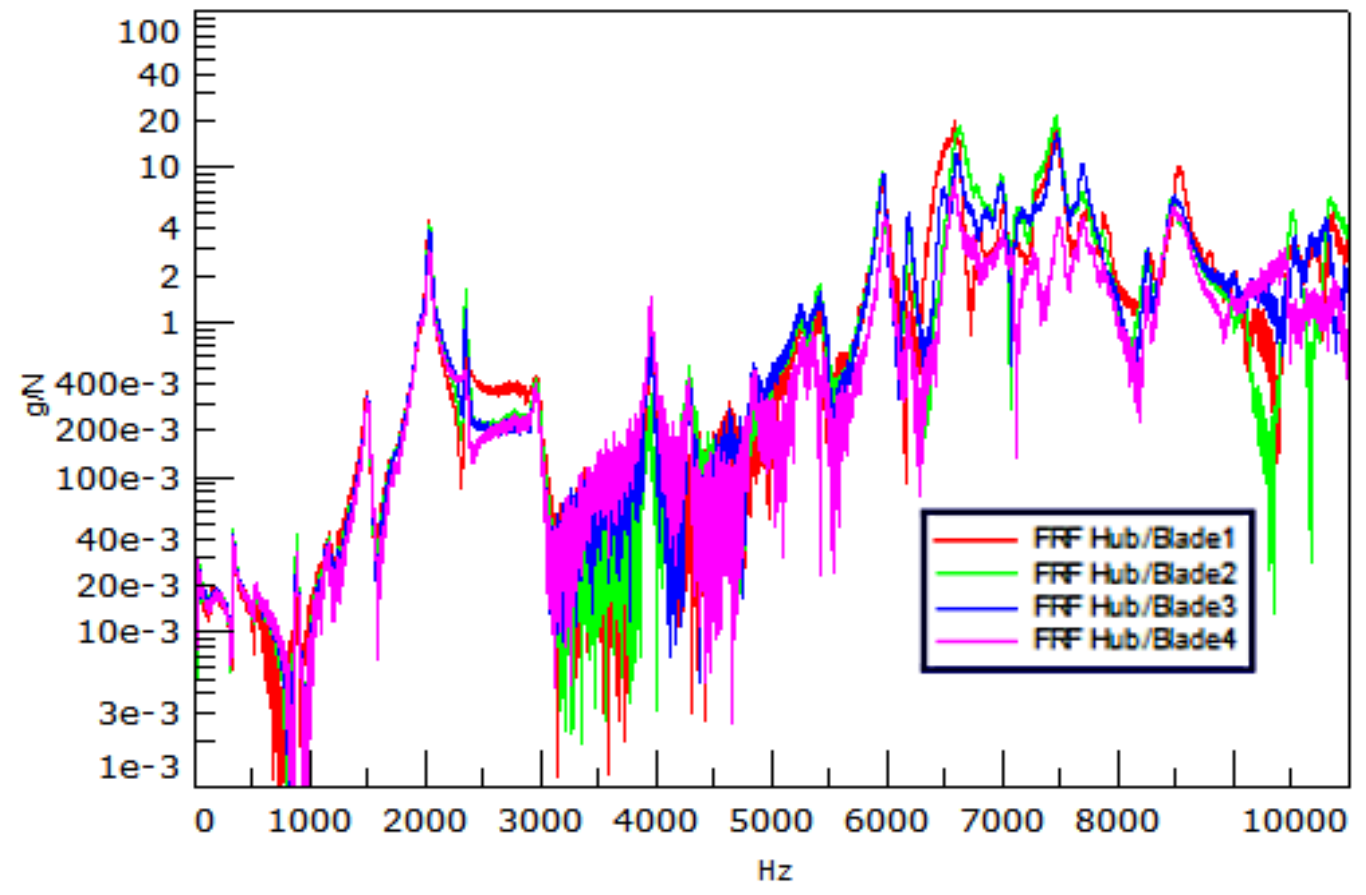

Figure 4.12: Comparison of frequency response functions of a reference point on the hub of the un-notched turbine of a noisy turbine with four trailing edge blades tested in ATF.

Operational Testing - To gain understanding of hydrodynamic performance and noise generation during the turbine noise event, a dynamometer test stand was utilized to perform operational testing. Kowalski, et al (2), developed a method to detect cavitation in torque converters tested on a dynamometer using nearfield acoustic measurements. A new acoustically treated dynamometer test fixture was employed by Robinette (1) to further study torque converter cavitation. The same dynamometer test stand and acoustic fixture were used to study turbine noise.

The test fixture utilizes two dynamometers to simulate automotive engine and transmission loading conditions. The acoustic test fixture is much larger than typically used for torque converter testing so that it can be filled with acoustic foam to assist in absorbing and insulating noise. Figure 4.13 shows pictures of the dynamometer test stand with both a typical torque converter test fixture and the acoustically treated fixture that was used for the cavitation experiments. On the right is a picture of the fixture opened to reveal a torque converter and acoustic foam. 


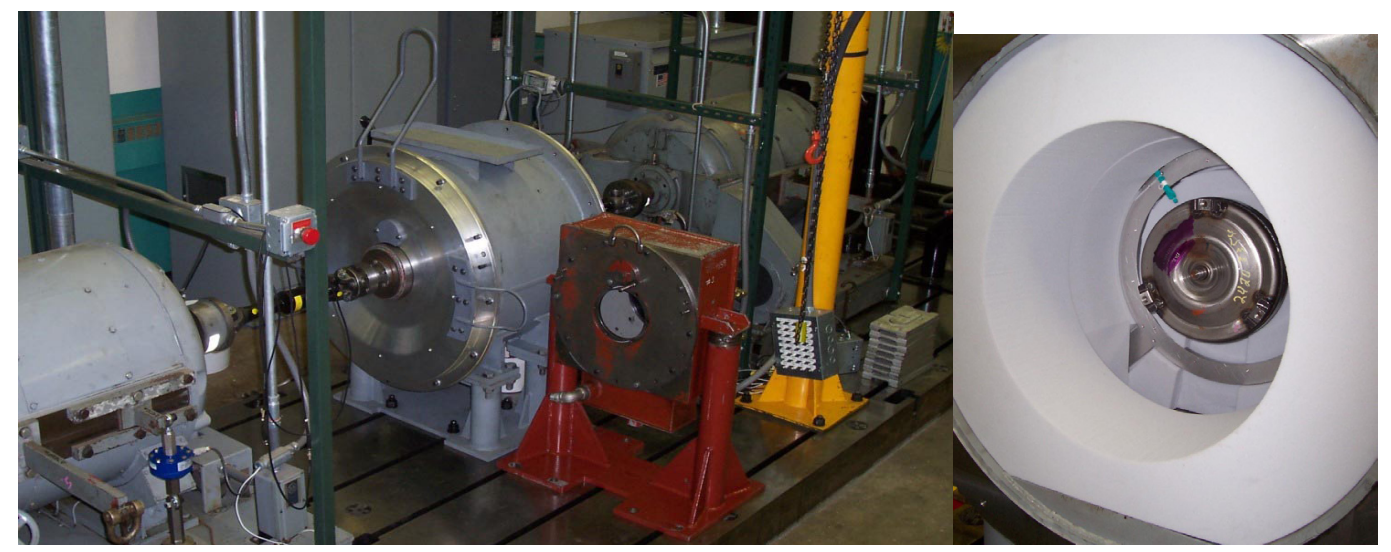

Figure 4.13: Left: Comparison of original torque converter dynamometer test fixture (Red) and acoustic test fixture (Gray). Right: Close up of opened acoustic test stand with acoustic foam. (1)

Test Procedure - For turbine noise testing, a standard dynamometer test procedure was developed to emulate torque converter performance during vehicle takeoff. The dynamometer test starts with a warm up and stabilization cycle. Then, the individual experiments begin with the torque converter pump being spun up to a specified constant torque. The turbine is rotated at a constant 500 RPM during this spin-up phase of the pump. Once the pump torque has settled out, the turbine is accelerated at a rate of 50 $\mathrm{RPM} / \mathrm{s}$ to a speed of $3000 \mathrm{RPM}$. During the test, all speeds, torques, pressures, temperatures, and flow rates are collected via the control system at a $10 \mathrm{~Hz}$ sample rate. Nearfield acoustic measurements are taken at a $51.2 \mathrm{kHz}$ sample rate. Pump and turbine speeds are also measured at $51.2 \mathrm{kHz}$ so that some reference may be made between the acoustic data and other test parameters. After a test is completed, a torque correction factor which includes dynamic torque losses due to the inertia of the entire dynamometer system is applied to the measured torques.

For most of the tests outlined in this paper, a suite of turbine data was acquired in addition to nearfield acoustic data. This included trailing edge blade acceleration, strain, and surface pressure data.

Dynamometer Tests - The turbine noise was known to be a high input torque phenomenon. To better understand the torque influence, an investigation was conducted to identify the effect of the input torque on the generated noise. Figure 4.14 is a waterfall 
plot of bandpass filtered SPL versus torque converter speed ratio for the range of input torques tested. Each increase in input torque increased the SR at which the noise began and ended. In addition, the peak noise level rose for each increase in input torque.

The individual torque plots in Figure 4.14 resemble the displacement plot of vortex lockin shown in Figure 4.6. The similarity could indicate that over the course of torque converter operation, the free stream velocity increases or decreases in such a way that the vortex frequency of the flow passes through the natural frequency of the turbine similar to the flow phenomenon depicted in Figure 4.6.

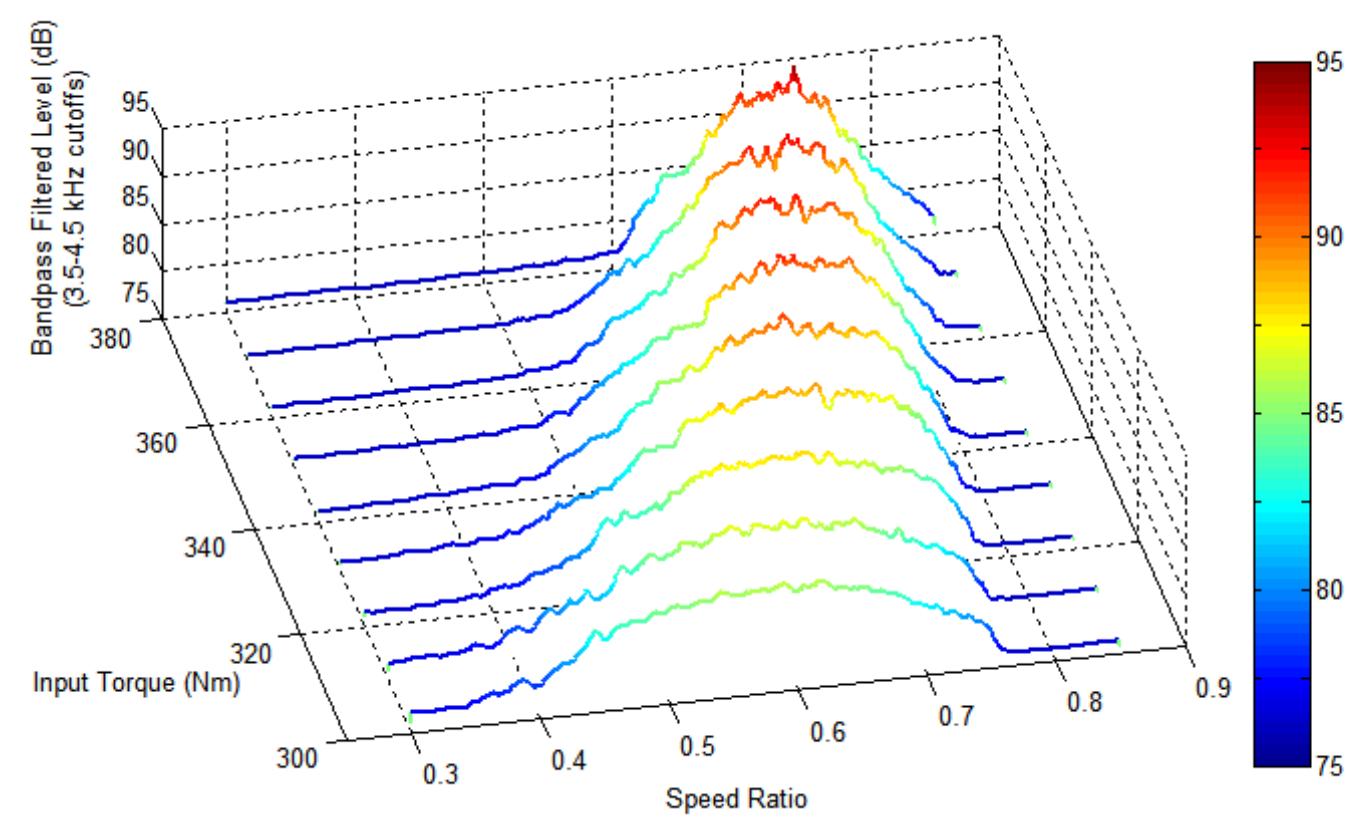

Figure 4.14: Comparison of Torque Converter turbine noise at various input torque steps.

Microwave Telemetry - To better understand the dynamics of the turbine noise phenomenon, the motion of and local pressure near the trailing edges of the turbine blades during operation were required. Therefore, a more invasive experimental technique was used. However, measurement difficulties stemmed from the fact that the point of interest was on a part rotating within and relative to another part within the torque converter. A wireless telemetry system was used to measure the parameters of the 
flow near the turbine's trailing edge. This wireless telemetry, designed by IR Telemetrics, employed a microwave technique of data transfer described by Sweger in (7).

Telemetry Pretest Experimentation - The phenomenon being measured was known to be affected by various modifications to the trailing edges of the turbine blades. Therefore, before proceeding to the actual testing, it was important to determine if alterations to the flow, due to the presence of transducers and wires on the turbine blades, would eliminate the turbine noise. Transducers were selected for small size, robustness, and ease of interface with the transmitter. Three attributes were selected to be measured; fluid pressure, blade strain, and blade acceleration. Telemetry wires and dummy transducers of similar size to the actual largest transducer were made from aluminum and placed on the turbine blades. Pictures of the altered turbine are shown in Figure 4.15. Dummy wires and transducers were placed on every blade to create a worst case test of the flow altering effects. Dynamometer tests were run first with an unaltered turbine, next with added dummy transducers, and finally with dummy transducers and wires. The resulting SPL curves are shown in Figure 4.16.
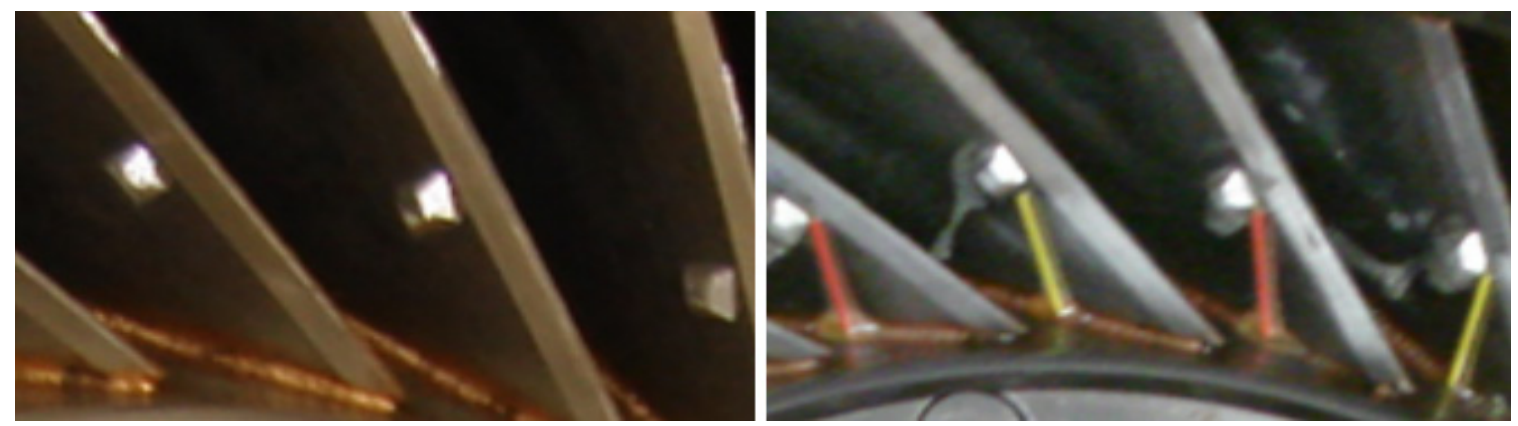

Figure 4.15: Photographs of un-notched turbine trailing edge with dummy transducers (Left) and wires combined with dummy transducers (Right).

As evident in Figure 4.16, the addition of the dummy transducers had a small effect on the noise generated, lowering the peak noise level by about $2 \mathrm{~dB}$. However, the addition of the wires significantly decreased the noise created by the turbine, lowering the peak noise level by almost $14 \mathrm{~dB}$. However, the dummy tests were performed with every blade of the turbine having an attached dummy block, while the actual telemetry turbine would only have seven transducers. As the turbine noise was still evident with a worst case 
dummy transducer/wire combination, it was determined that the telemetry testing could proceed.

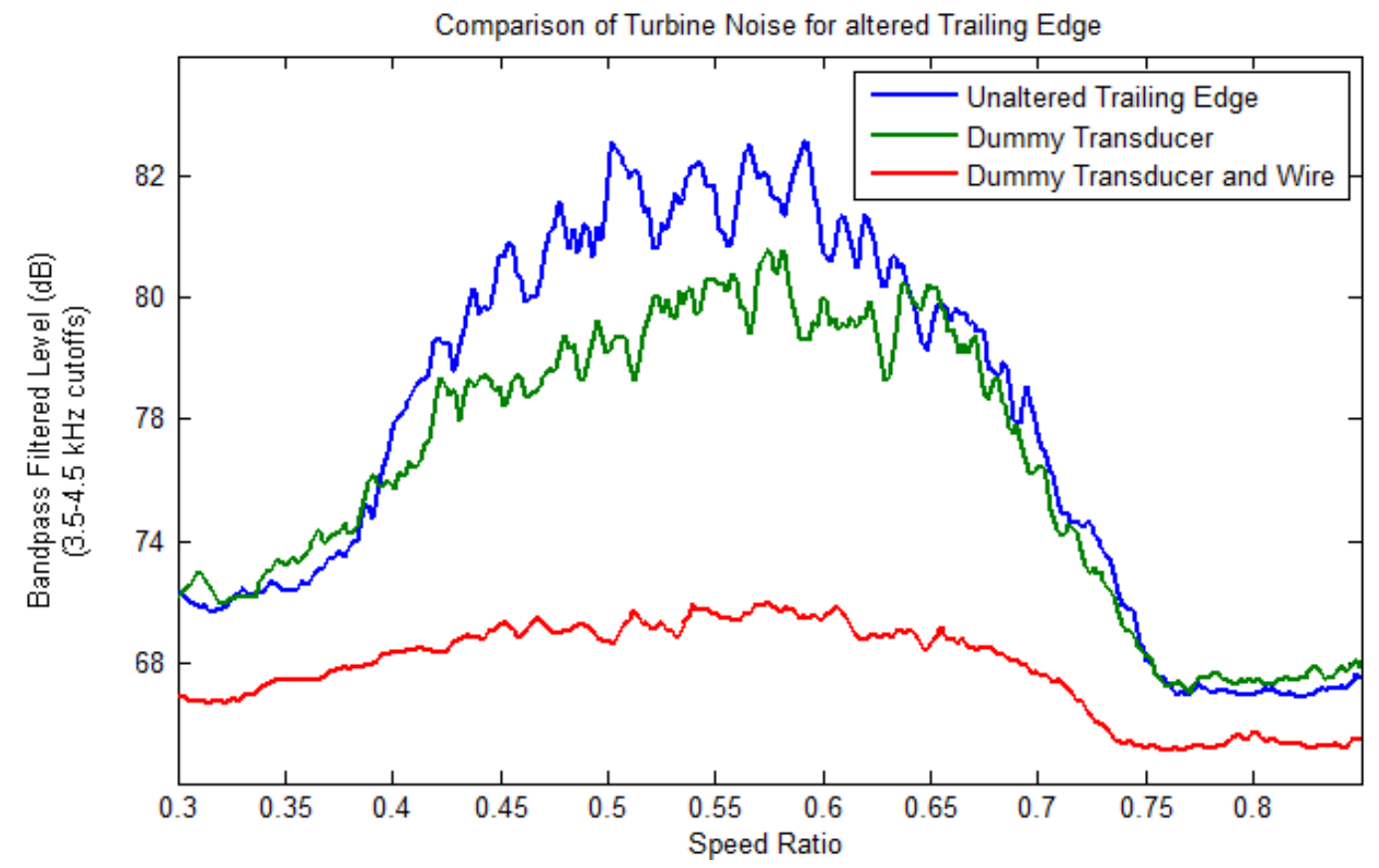

Figure 4.16: SPL plot comparing test alterations to turbine trailing edge.

The fact that the turbine noise diminished with the presence of dummy transducers and wires is in line with the vortex shedding hypothesis. According to Blevins (5), creating some disturbance in the flow field near the point where vortices form prevents them from coupling with the structure. This could be why the noise is reduced but not completely eliminated by the transducer wires. The resulting alteration to flow is not large enough to either eliminate the vortices or completely decouple them from the structure.

Microwave Telemetry Preparation - Several modifications to the existing torque converter assembly were required for installation and operation of the telemetry system. Since a torque converter is completely housed inside of a metal shell, creating a Faraday's Cage, an opening in the metal is required to allow the transmission of the microwaves to the receivers. A polycarbonate extension ring for a bolt together torque converter was designed to provide an outlet path for microwave transmission. The ring, shown in Figure 4.17, permits the microwaves to travel from the transmitter inside the torque converter to the external receiver. A second function of the polycarbonate 
extension ring is to allow enough clearance for the microwave transmitter to fit inside the torque converter. A cross-sectional view of the assembled torque converter, polycarbonate extension ring, and microwave telemetry is shown in Figure 4.18.

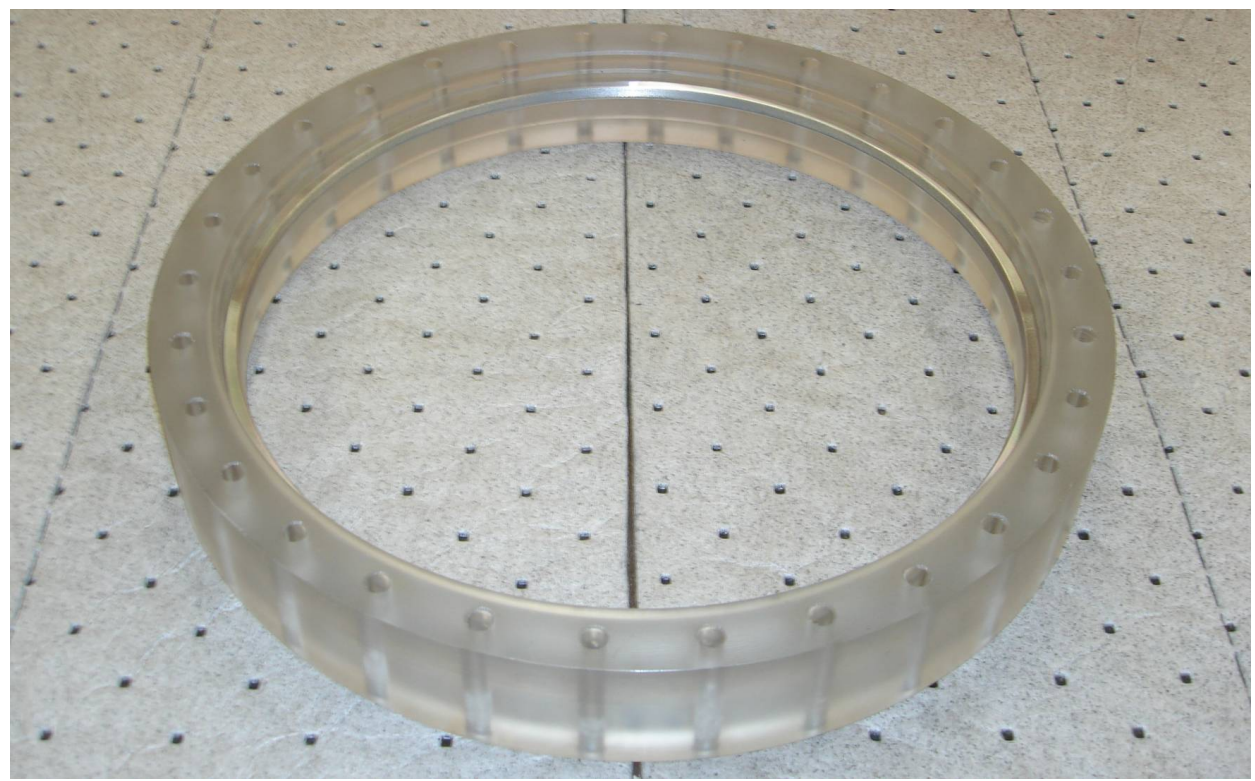

Figure 4.17: Polycarbonate Extension Ring used to facilitate microwave telemetry measurements.

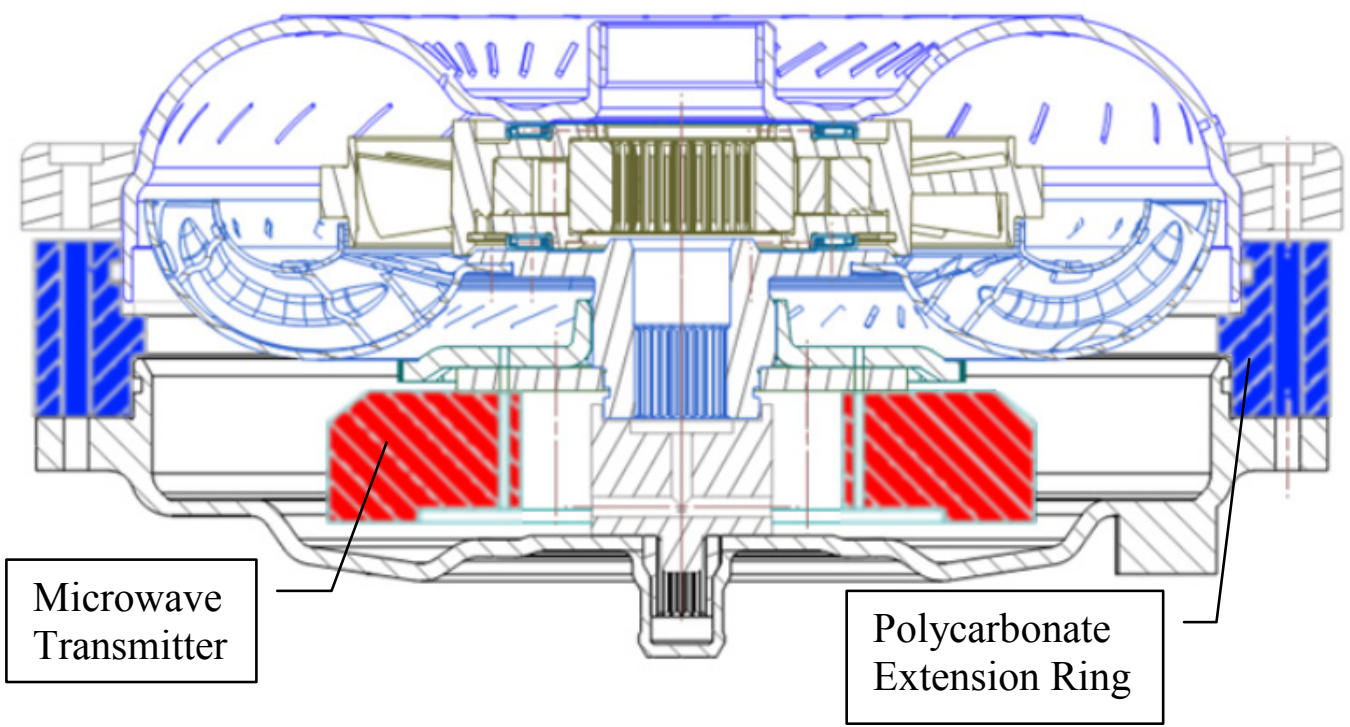

Figure 4.18: Cross-section of assembled telemetry torque converter. The polycarbonate extension ring and the microwave transmitter are annotated. 


\subsection{Analysis}

Telemetry Data Analysis - Figure 4.19 shows accelerometer data measured at the trailing edge of a turbine blade from a SR test with $350 \mathrm{Nm}$ input torque. The top plot contains measurements from the un-notched turbine and the bottom plot from the notched turbine. A clear band of motion at $4 \mathrm{kHz}$ is shown for both sets of data. However, the amount of motion is much greater on the un-notched turbine (ten times greater). This indicates that at the frequency of interest, the trailing edge of the turbine is indeed moving, and could be the source of the noise. With respect to Vortex shedding, recall that the impact data demonstrated that a natural frequency of the turbine blades exists at 4 $\mathrm{kHz}$ for both the notched and un-notched turbines in ATF. This means that the notch affects the flow in some way other than modifying the structural parameters of the turbine's trailing edge, and that blade motion occurs at a natural frequency for both turbines. The fact that the disturbance occurs at a natural frequency is a characteristic of vortex shedding with lock-in. With the notched turbine, the excitation is just not great enough to be detected.

For Figure 4.19 through 21, the responses are plotted in Decibels $(\mathrm{dB})$ to allow the data to better stand out and facilitate data comparisons. The decibel values are simply the log of the value measured divided by some reference value. In the cases of acceleration, fluid

pressure, and strain, the reference values used were $20 \mu \mathrm{m} / \mathrm{s}^{2}, 2 \mathrm{e}-5 \mathrm{psi}$, and $2 \mathrm{e}-5 \mu \mathrm{strain}$ respectively. 

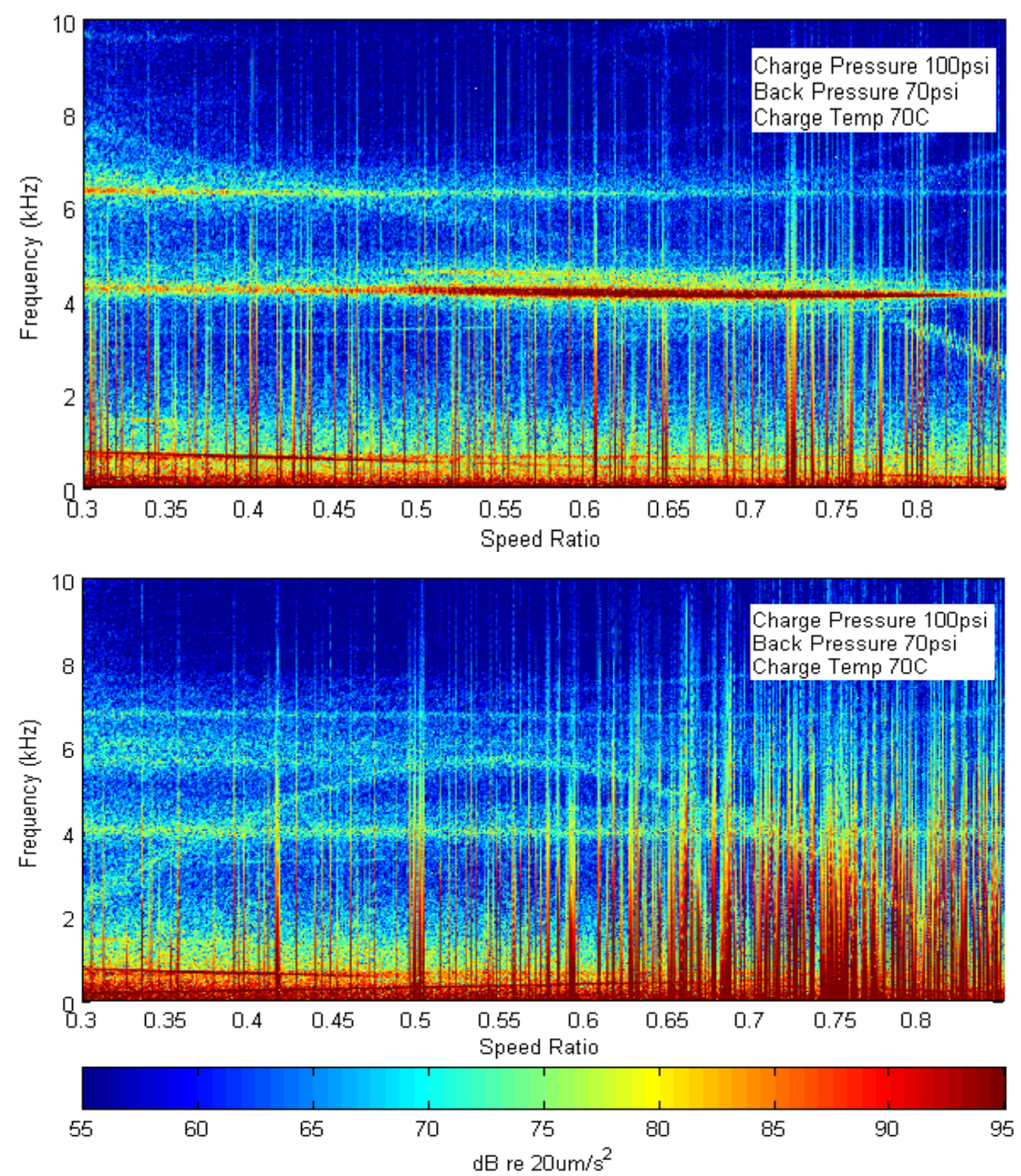

Figure 4.19: Spectrogram of trailing edge accelerometer measurement on automotive torque converters equipped with an un-notched (Top) and notched (Bottom) trailing edge turbine during simulated vehicle launch with an input torque of $350 \mathrm{Nm}$.

Normally bright lines in color maps, like those seen diagonally in the top part of Figure 4.19 and in an arc shape in the bottom part, are an indication of some vibratory phenomenon that is changing over time. However, after inspecting the content of the raw time signal it was determined that these lines were aliased artifacts of the telemetry signal. This is a known effect of the telemetry, and, once identified, can be ignored. Another known artifact of telemetry data is the bright vertical lines in any telemetry plot. 
These lines are a result of signal dropouts that occur from a poor signal path or excess noise at the telemetry carrier frequency, and can also be ignored.

Figure 4.20 and Figure 4.21 show the trailing edge fluid pressure response and blade strain at $350 \mathrm{Nm}$ input torque of the un-notched and notched turbines, respectively top and bottom. As with the accelerometer measurements, the pressure fluctuation and strain around $4 \mathrm{kHz}$ are evident in the un-notched turbine. In the notched turbine no pressure fluctuation is detectable above the background noise, while only minimal strain is observed. These results also follow the vortex induced vibration hypothesis. The minimal strain and absence of pressure fluctuations could mean that the addition of notches prevents the flow from forming vortices about the trailing edge of the turbine. 

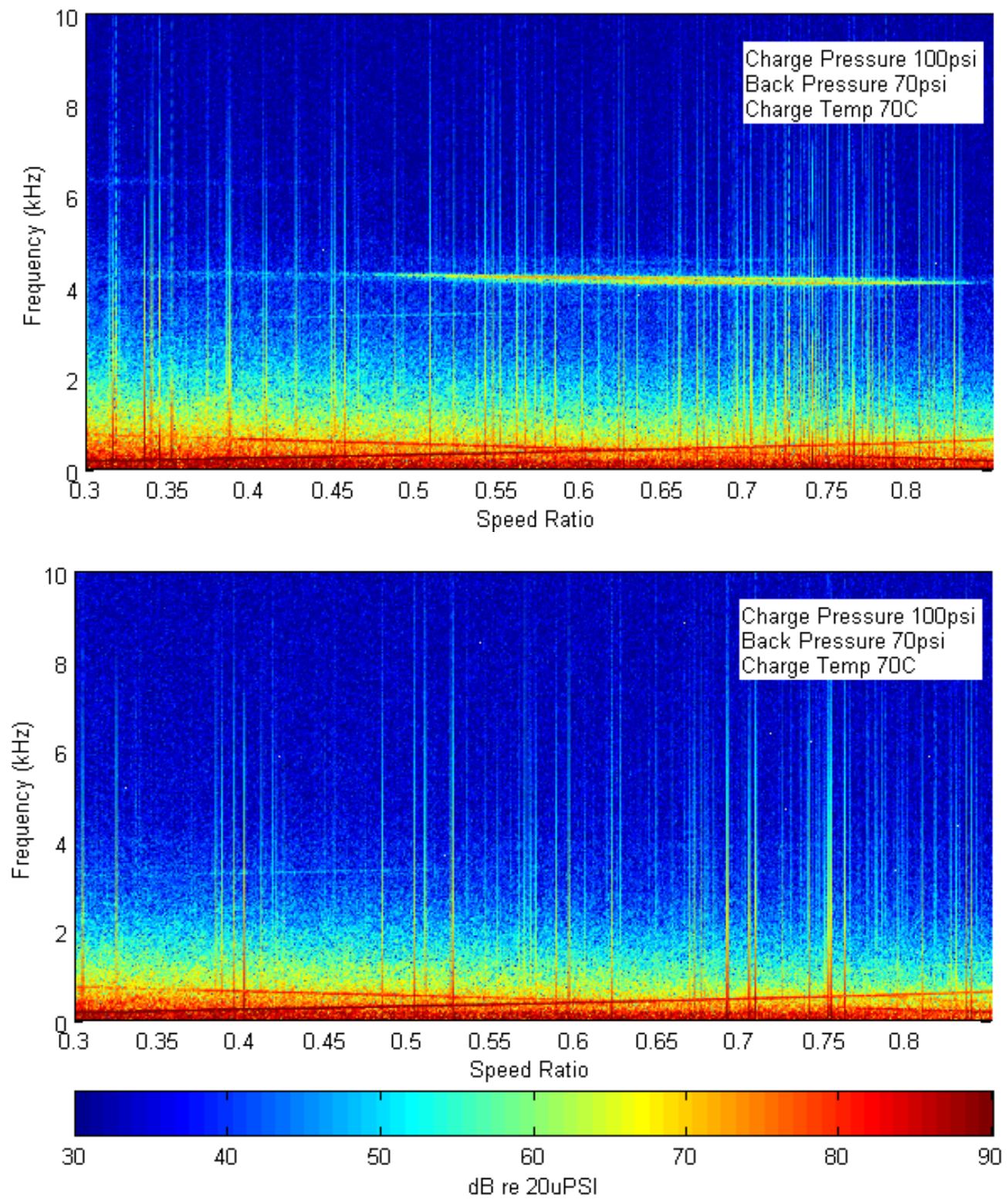

Figure 4.20: Spectrogram of trailing edge pressure measurement on automotive torque converter equipped with an un-notched (Top) and notched (Bottom) trailing edge turbine during simulated vehicle launch with an input torque of $350 \mathrm{Nm}$. 

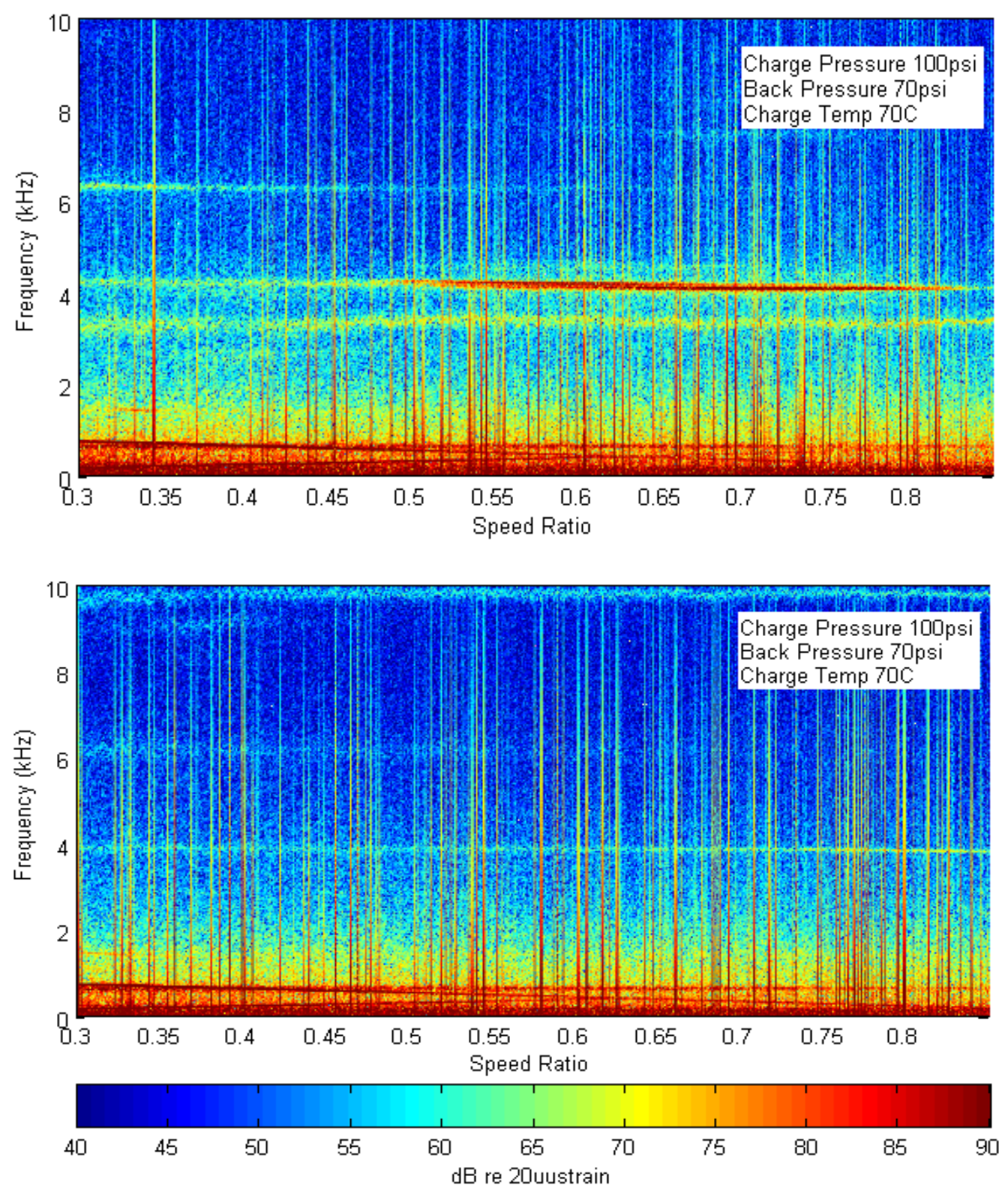

Figure 4.21: Spectrogram of trailing edge strain gage measurement on automotive torque converter equipped with an un-notched (Top) and notched (Bottom) trailing edge turbine during simulated vehicle launch with an input torque of $350 \mathrm{Nm}$.

Turbine Exit Flow Velocity - Further analysis of vortex shedding required determining the average velocity of the ATF exiting the turbine. Referring to Equation 3, free stream velocity can be used to calculate the vortex frequency from the Strouhal Number of a given structure. During the course of these experiments, however, Strouhal Number was 
unknown for automotive torque converters. The goal, then, became to formulate the Strouhal Number with what was known. This estimated Strouhal Number would then be used to calculate the vortex frequency, and thereby ascertain whether or not the turbine was operating near a lock-in condition during the noise phenomenon.

Unfortunately there exists no means by which free stream velocity at the turbine exit could be measured directly during operational conditions. Therefore, a computational fluid dynamics (CFD) model was created by the GM Powertrain Transmission Systems and CFD Analysis Group using the given torque converter geometry and the test parameters. Flow velocity results from the CFD simulations were used to calculate the average toroidal flow velocity exiting the turbine for a limited matrix of input torques and speed ratio conditions. That velocity data was then used to estimate the turbine exit velocity over speed ratio at each input torque tested. The results are shown in Figure 4.22.

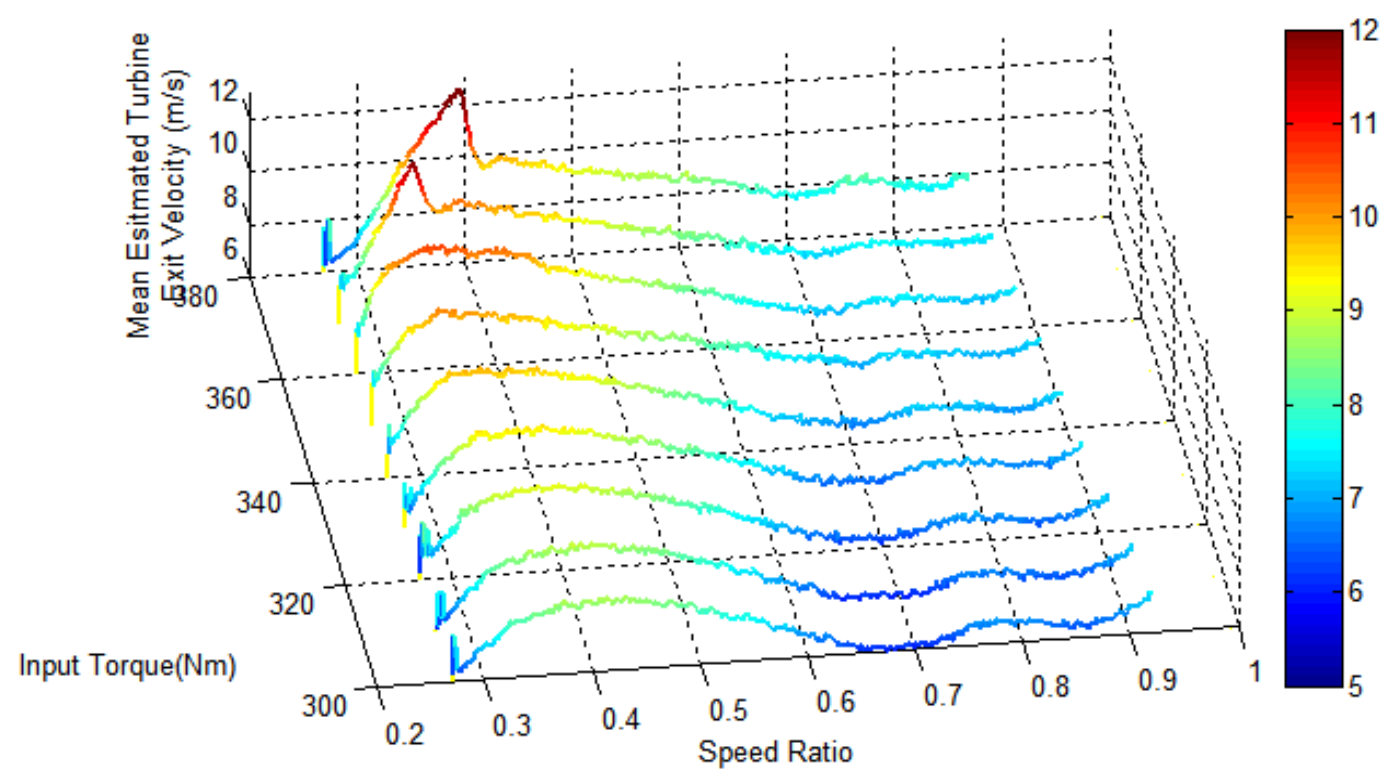

Figure 4.22: Estimated turbine exit mean velocity at each tested input torque over speed ratio.

The estimated flow velocities and the center frequency of the noise band excited were used to calculate the Strouhal Number. The geometry component in Equation 3 is usually the thickness of the structure normal to the direction of flow. For the turbine, this is the 
blade trailing edge thickness. A Strouhal Number of 0.28 was calculated and was assumed to be constant across all of the tested conditions. This fits very well in the range of known Strouhal Numbers for existing geometries. The top curve in Figure 4.23 shows a plot of the resulting vortex frequency curve versus SR calculated from the estimated turbine exit velocities using 0.28 as the Strouhal Number, and using $0.6 \mathrm{~mm}$ as the characteristic length. The lower curve in Figure 4.23 is the filtered sound pressure level curve from the $350 \mathrm{Nm}$ input torque test. As speed ratio increases and the vortex frequency nears $4 \mathrm{kHz}$, the noise begins to develop (this occurs at about $0.5 \mathrm{SR}$ ). The vortex frequency continues down to just below $3.5 \mathrm{kHz}$ and stays constant through the rest of the experiment. A frequency of $3.5 \mathrm{kHz}$ might be just far enough away from the natural frequency of the structure that the vortices begin to decouple and allow the noise to dissipate, similar to the lock-in flow phenomenon depicted in Figure 4.6.
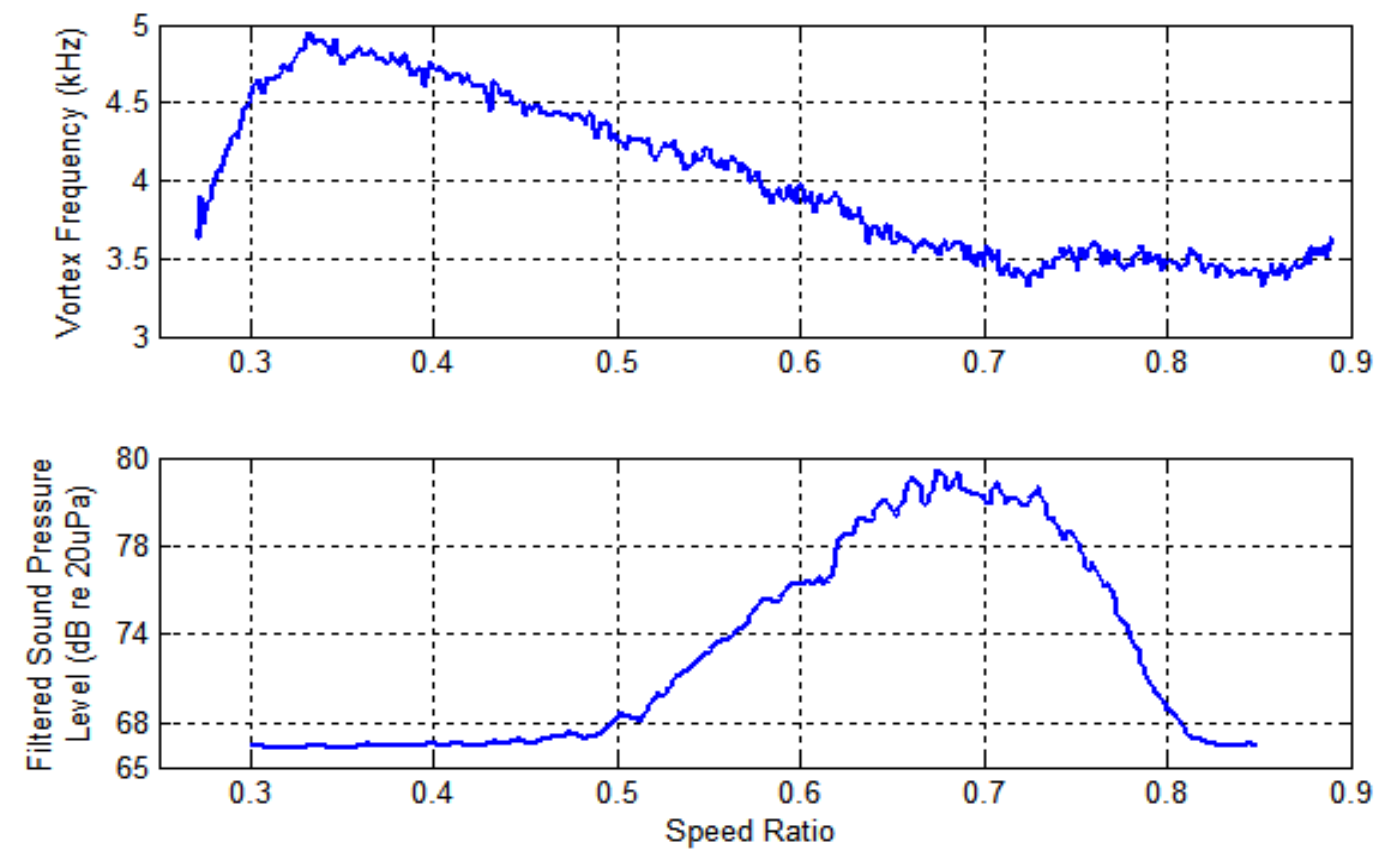

Figure 4.23: Plots of estimated vortex frequency (Top) and filtered sound pressure level (Bottom) over speed ratio. The noise data was taken during simulated vehicle launch testing on dynamometer with an input torque of $350 \mathrm{Nm}$. The vortex frequency was calculated using the turbine exit velocity curve for $350 \mathrm{Nm}$, a trailing edge thickness of $.6 \mathrm{~mm}$, and a Strouhal Number of .28 over the entire test.

It is interesting to note that the exit flow velocity, and therefore the vortex frequency, of the turbine do not vary much once the onset of the noise occurs. This could explain why 
the noise is maintained for such a long duration of speed ratio. Once lock-in occurs, the flow velocity does not deviate far enough to allow the vortex frequency to break away from the natural frequency of the structure.

Noise Testing Torque Converter Elements - Additional alterations to the torque converter were made to further test the Vortex shedding theory. It is well understood from Equation 3 that changing flow velocity changes the vortex frequency. However, for a single converter operating at a constant input torque with the turbine accelerated at a given rate (as is performed during the experimentation described here), the flow velocity profile over speed ratio is assumed to be repeatable with the values shown in Figure 4.22. Therefore, to alter the flow velocity profile over speed ratio, the blade geometries of the pump and stator were changed. Figure 4.24 shows a comparison of the noise versus speed ratio of three converters, all using the same turbine but with different combinations of pump and stator. All of these measurements were taken with a microphone, and all three converters were operated with a $300 \mathrm{Nm}$ input torque.

Two trends can be observed. First, it appears that the stator affects the flow in a way which determines the duration of the noise with respect to speed ratio. In the vortex shedding hypothesis, this means that the flow velocities favorable to the vortex frequency of interest are sustained for either a longer or a shorter range of speed ratios depending on the stator in use. Second, changing the pump of the converter appears to have the effect of altering the magnitude of the turbine noise. 


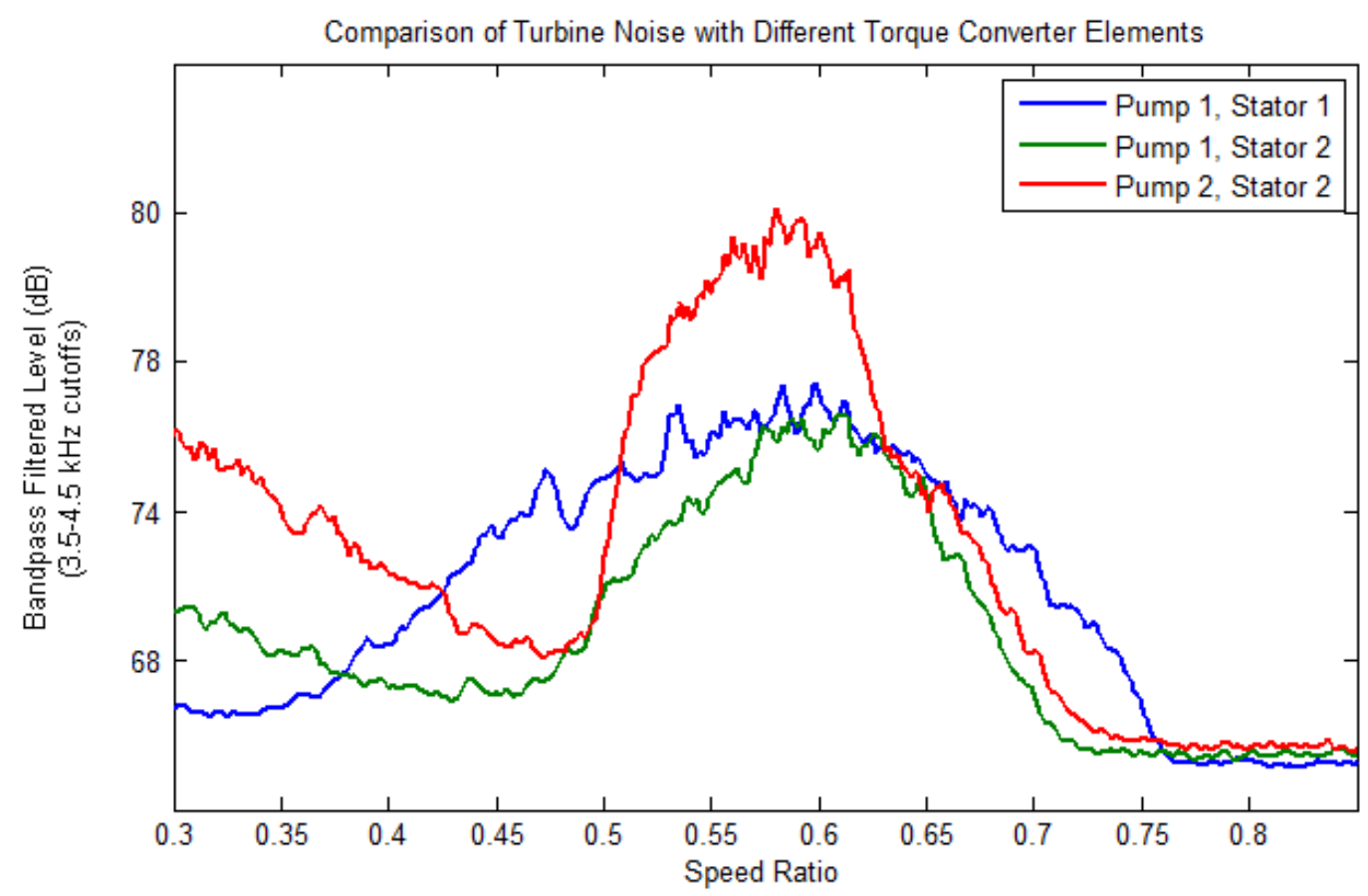

Figure 4.24: SPL plot comparing noise level in converters with different stator and pump combinations. Each of the tests shown was run at $300 \mathrm{Nm}$ input torque.

Turbine Blade Trailing Edge Thickness - Following Equation 3 once again, altering the characteristic length of the geometry hypothesized to be creating the vortices should also alter the vortex frequency. For this experiment, another turbine was selected and tested for turbine noise. The blade trailing edges of this turbine were then ground thinner to a thickness where the vortex frequency was expected to interact with a higher turbine natural frequency and create a similar noise at the new frequency.

Figure 4.25, shows the sound pressure level color maps from testing the un-notched turbine and testing the same turbine after the trailing edge modification. It is evident that making the trailing edge thinner did indeed alter the noise, but by eliminating it instead of changing its frequency. One possibility for the noise disappearance could be that even if the vortices are still occurring at the higher vortex frequency, they might not couple with a vibration mode of the turbine to excite it acoustically. The disturbance therefore would be undetectable with the microphone. 

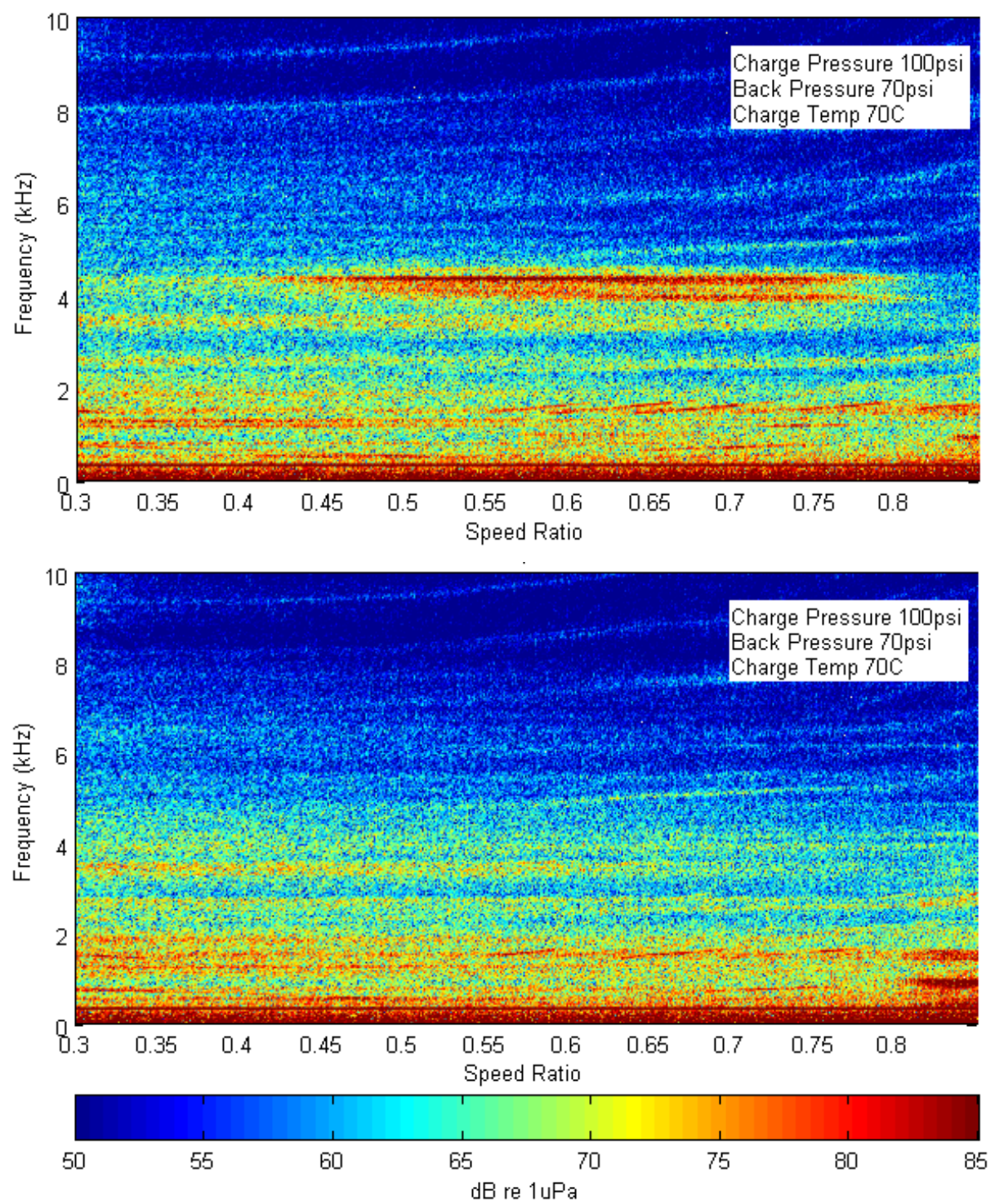

Figure 4.25: Color maps comparing a stock turbine noise (Top) with the same turbine after machining the trailing edge thinner (Bottom), both tests at $350 \mathrm{Nm}$ input torque

\subsection{Conclusions}

In order to investigate the root cause of the turbine noise phenomenon, numerous modifications to the torque converter turbine and to operating conditions were performed to evaluate the effects on turbine noise. The following observations from the acoustic and blade vibration measurements and analysis confirm that the turbine noise phenomenon follows the conditions for Von Karman Vortex Shedding Lock-in. 
- Acoustic and vibration measurements show that turbine noise was generated at a frequency between 3.5 and $4.5 \mathrm{kHz}$. Impact testing of the turbine in ATF showed a natural frequency near $4 \mathrm{kHz}$. The close match of frequencies supports a Vortex Shedding Lock-in condition.

- Increasing input torque results in an upward shift of the turbine noise relative to speed ratio and in higher noise levels. The change in free stream velocity exiting the turbine that results from the change in input torque alters the Strouhal Number and the proximity of vortex frequency to turbine natural frequency. The resulting noise versus input torque and speed ratio pattern resembles the lock-in and release pattern of Vortex Shedding.

- Acoustic measurements and turbine blade measurements for acceleration, strain, and pressure confirmed that altering the trailing edge of a noisy turbine either reduces or completely eliminates the noise. The blade geometry modifications from the notch and telemetry transducers alter the flow enough to reduce or eliminate the vortices or decouple them from the structure. The effect is similar to claims made for vortex suppression features added to other types of turbomachinery such as computer fans.

- Free stream velocities (and thus Strouhal number and vortex frequency) were modified by testing several combinations of pumps and stators. Similar to changing input torque, the alterations to pump and stator blade geometries either shifted the noise duration with respect to torque converter speed ratio, or changed the level of noise.

- The structure characteristic length for Strouhal number was changed by reducing the turbine trailing edge thickness. The modified Strouhal number was expected to shift the vortex frequency and turbine noise to a higher turbine natural frequency. The fact that the noise was eliminated instead could mean that the vortices could not couple with a turbine mode to excite it acoustically.

- The value of Strouhal number for the turbine noise phenomenon was estimated from CFD simulations of free stream velocity exiting the turbine for a range of operating conditions. The resulting estimate was a constant of 0.28 which fits well with known Strouhal Numbers of other similar geometries. The estimated Strouhal Number was 
subsequently used to calculate turbine vortex frequency versus speed ratio. The results in Figure 4.23 show that the onset of the noise coincided with vortex frequency passing near $4 \mathrm{kHz}$. Therefore, the simulated characteristics of flow exiting the turbine during the turbine noise phenomenon follow requirements for vortex lockin.

\section{Acknowledgement}

The writers would like to thank Ibraheem Shaik from the GM Powertrain Systems and CFD Analysis Group for his contribution of CFD simulations used to estimate torque converter flow velocities at the turbine exit.

\subsection{References}

(1) Robinette DL. Detecting and Predicting the onset of Cavitation in Automotive Torque Converters. PhD Dissertation, Michigan Technological University. Ann Arbor: ProQuest/UMI. 2007.

(2) Kowalski D, Anderson CL, Blough JR. Cavitation Detection in Automotive Torque Converters Using Nearfield Acoustical Measurements. SAE-NVH. 5/16/2005; 200501- 2516 .

(3) La Rosa Siqueira C. Wikipedia. File:Vortext-street-animation.gif. 2005. http://en.wikipedia.org/wiki/File:Vortex-street-animation.gif.

(4) Blevins R. Flow Induced Vibration. Krieger Publishing Company. 2001.

(5) Noctua Fan Company. Nine Blade Design with Vortex-Control Notches. 2012; http://www.noctua.at/main.php?show=nine_blade_design\&lng=en.

(6) Walber C, Blough JR, Anderson CL, Johnson MA. Measuring and Comparing Frequency Response Functions of Torque Converter Turbines Submerged In Transmission Fluid. SAE-NVH. 5/17/2011; 2011-01-1662.

(7) Sweger P, Anderson CL, Blough JR. Measurements of Strain on $310 \mathrm{Mm}$ Converter Turbine Blade. International Journal of Rotating Machinery. 2004; Volume 10, No 1: pp. 55-63.

(8) Mekkes J, Anderson CL, Narain A. Static Pressure Measurements and Cavitation Signatures on the Nose of a Torque Convertor's Stator Blade. ISROMAC. 2004.

(9) Robinette DL, Schweitzer JM, Maddock DG, Anderson CL, Blough JR, Johnson MA. Predicting the Onset of Cavitation in Automotive Torque Converters-Part I: Designs with Geometric Similitude. International Journal of Rotating Machinery. 2008; Article ID 803940. 


\section{Chapter 5 - Conclusions}

This chapter will discuss the findings of the two primary projects, desinent cavitation detection during vehicle launch and the turbine noise phenomenon. Several recommendations will be made with regards to torque converter research in general.

\subsection{Experimental Desinent Cavitation Conclusions}

The observations made with relation to desinent cavitation are based on nearfield acoustic measurements and analysis collected during constant input torque turbine speed sweeps. The findings of this study are summarized here:

1) A dimensionless group created from the product of dimensionless input torque and speed ratio was selected as the dimensionless response in an effort to be able to relate both quantities to the torque converter design parameters as well as the varying operating conditions during vehicle launch. This quantity was named dimensionless rotational power.

2) Increasing charge pressure and decreasing the temperature of the cooling flow reduces the duration of cavitation occurring in the torque converter; that is by ending cavitation at a lower speed ratio. This is in accordance with the stall cavitation relation.

3) Increasing torque input to the torque converter increases the duration of cavitation.

4) The response surface models that were created based on the converters having similar geometric properties (Torus aspect ratio) or similar operating capacities (Unit input speed) are capable of predicting the dimensionless rotational power of the converter at the point of desinent cavitation within a \%RMSE of 10.8 and 11.6 percent respectively.

5) The general model developed from test results for all the converters tested is capable of predicting dimensionless rotational power at desinent cavitation with a 14.4 $\% R M S E$. 


\subsection{Turbine Noise Study Conclusions}

A summary of the discoveries made during the turbine noise study are listed below.

1) Some turbines are more susceptible to the noise phenomenon than others. It is believed that the sensitivity is due to a combination of braze quality, hub attachment, and trailing edge finish and thickness.

2) The turbine noise shifts to higher speed ratios when a higher input torque is applied.

3) Altering the trailing edge of a turbine which is noisy either reduces the noise or eliminates it completely. This includes the addition of trip wires, notches, dummy blocks, as well as altering the taper at the trailing edge of the turbine.

4) Some turbines generate the noise when paired with some stator and pump combinations, but not in others.

5) The frequency at which the noise occurs does not shift with any of the alterations discussed in the previous conclusions.

6) Based on previous conclusions, it is believed that vortex shedding is the most likely cause of the noise phenomenon. The frequency at which the vortices form relates very closely to both a natural frequency of the turbine trailing edge blades as well as the estimated vortex frequency during that point of torque converter operation.

7) Thus far it has been impossible to determine by measuring frequency response or any other static non-operating tests whether or not a turbine will be noisy or quiet.

\subsection{General Automotive Torque Converter Recommendations}

The study discussed here has raised some additional questions that require additional research to fully understand. The following list contains work that is recommended to be done in the future to better understand automotive torque converters in general, as well as the two primary investigations discussed here.

1) Create a bench-top test to perform flow measurements on portions of torque converter elements to understand the flow interactions inside the individual passages of each specific element (pump, stator, and turbine). These experiments should take into 
account not only flow speed, but also angle of attack of the flow on the leading edge of the blades of that particular element.

2) As a part of a bench-top turbine test, determine if Von-Karman Vortices can be seen shedding off of the trailing edges of the turbine blades.

3) Select one torque converter where the operational parameters are very well known, and perform a full vehicle launch simulation, including transmission shifts and throttle adjustments, using the dynamometer test stand and correlate the findings to actual vehicle launch data. Include nearfield acoustic measurements in both experiments.

4) Develop a full computational fluid dynamics (CFD) model of the flow inside the torque converter. This model should be correlated to not only steady state torque converter testing, but also speed sweep data and the testing performed in the benchtop experiment mentioned previously. If possible, a multiphase flow simulation should be included in this model in order to further increase understanding of cavitation and its effect on the operation of the torque converter.

5) Develop and perform a flow visualization experiment on an entire automotive torque converter assembly. The CFD model should also be correlated to the bench-top experimentation to be sure that all information garnered from the model is as accurate as possible.

6) Perform a full transfer path analysis on the torque converter-flex shaft-crank shaft vibration path during simulated operation. Use both axial force transducers and in line torque cells at each interface to measure the forces actually being imparted on the engine by the torque converter.

7) Perform more clutch engagement experimentation. Include clutch engagement in the transfer path analysis already discussed, but also include acoustic measurements during the experiment to determine if locking the clutch creates additional noise. This may be more important with the advent of launch converters as clutch chatter may become more prevalent. 


\section{References}

(1) Robinette DL. Detecting and Predicting the onset of Cavitation in Automotive Torque Converters. PhD Dissertation, Michigan Technological University. Ann Arbor: ProQuest/UMI. 2007.

(2) Arakeri V. Cavitation Inception. Proc. Indian Acad. Sci. 1979; Vol. C2, Pt. 2: pp. $149-177$.

(3) Mekkes J, Anderson CL, Narain A. Static Pressure Measurements on the Nose of a Torque Converter Stator during Cavitation. ISROMAC. 2004.

(4) Zeng L, Anderson CL, Sweger PO, Narain A. Experimental Investigation of Cavitation Signatures in an Automotive Torque Converter Using a Microwave Telemetry Technique. ISORMAC. 2002.

(5) Blander M, Katz JL. Bubble Nucleation in Liquids. American Institute of Chemical Engineers. 1975; Vol. 21, No. 5: pp. 833-847.

(6) Reynolds O. On The Dynamical Theory of Incompressible Viscous Fluids and the Determination of the Criterion. Proceedings of the Royal Society of London. 1895.

(7) Strasberg M, Taylor D. Propeller Cavitation Noise after 35 Years of Study. Noise and Fluids Engineering. 1977.

(8) Kowalski D, Anderson CL, Blough JR. Cavitation Detection in Automotive Torque Converters Using Nearfield Acoustical Measurements. SAE-NVH. 5/16/2005; 2005-01-2516.

(9) Robinette DL, Anderson CL, Blough JR, Johnson MA, Maddock DG, Schweitzer JM. Characterizing the Effect of Automotive Torque Converter Design Parameters on the Onset of Cavitation at Stall. SAE-NVH. 5/15/2007; 2007-01-2231.

(10) Alfayez L, Mba D, Dyson G. The Application of Acoustic Emission for Detecting Incipient Cavitation and Best Efficiency Point of a $60 \mathrm{Kw}$ Centrifugal Pump: Case Study. NDT \& E International. 2005; Vol. 38, Issue 5: pp. 354-358.

(11) Barna G. IR Telemetrics. 2010.

(12) Robinette DL, Schweitzer JM, Maddock DG, Anderson CL, Blough JR, Johnson MA. Predicting the Onset of Cavitation in Automotive Torque Converters-Part II: A Generalized Model. ISORMAC. 2008; Article ID 312753. 
(13) Kowalski D, Anderson CL, Blough JR. Cavitation Prediction in Automotive Torque Converters. SAE-NVH. 2005; 2005-01-2557.

(14) La Rosa Siqueira C. Wikipedia. File:Vortext-street-animation.gif. 2005. http://en.wikipedia.org/wiki/File:Vortex-street-animation.gif.

(15) Blevins R. Flow Induced Vibration. Krieger Publishing Company. 2001

(16) Noctua Fan Company. Nine Blade Design with Vortex-Control Notches. 2012; http://www.noctua.at/main.php?show=nine_blade_design\&lng=en.

(17) Raabe J. Cavitation Effects in Turbomachinery - European Experiences: Cavitation State of Knowledge. ASME Fluid Engineering Div. 6/1969.

(18) Sweger P, Anderson CL, Blough JR. Measurements of Strain on $310 \mathrm{Mm}$ Converter Turbine Blade. International Journal of Rotating Machinery. 2004; Volume 10, No 1: pp. 55-63.

(19) Wen Y, Henry M. Time Frequency Characteristics of the Vibroacoustic Signal of Hydrodynamic Cavitation. ASME Journal of Vibration and Acoustics. 2002; Vol 124: pp. 469-476.

(20) Arakeri VH, Acosta AJ. Viscous Effects in the Inception of Cavitation on Axisymmetric Bodies. ASME Journal of Fluids Engineering. 12/1973; pp. 519527.

(21) Baldassarre A, DeLucia M, Nesi P. Real-Time Detection of Cavitation for Hydraulic Turbomachines. Real-Time Imaging. 1998; pp. 403-416.

(22) Blake WK, Wolpert MJ, Geib FE. Cavitation Noise As Influenced By Boundary Layer Development on a Hydrofoil. Journal of Fluid Mechanics. 1977; Vol. 80: pp. 617-640.

(23) Brennen CE. Cavitation and Bubble Dynamics. Oxford University Press. 1995; .

(24) Caron JF, Farhat M, Avellan F. Physical Investigation of the Cavitation Phenomenon. 4th International Symposium on Cavitation. 6/2001.

(25) Chahine GL. Nuclei Effects on Cavitation Inception and Noise. 25th Symposium on Naval Hydrodynamics. 8/2004.

(26) Chen Y, Heister SD. Two-Phase Modeling of Cavitated Flows. Computers and Fluids. 1995; Vol. 24, No. 7: pp. 799-809. 
(27) Gu W, He Y, Hu T. Transcritical Patterns of Cavitating Flow and Trends of Acoustic Level. ASME. 2001; Vol. 123: pp. 850-857.

(28) Guennoun F, Farhat M, Bouziad YA, Avellan F. Experimental Investigation of a Particular Traveling Bubble Cavitation. 5th International Symposium on Cavitation. 2003.

(29) Knapp RT, Hammitt JW, Daily FG. Cavitation. Engineering Societies Monographs. 1970.

(30) Kunz R, Boger D, Stinebring D, Checzewski T. A Preconditioned Navier-Stokes Method for Two-Phase Flows with Application to Cavitation Prediction. Computers and Fluids. 2000; Vol. 29: pp. 849-875.

(31) Martin CS, Medlarz H, Wiggert DC, Brennen C. Cavitation Inception in Spool Valves. Transaction of ASME. 1981; Vol. 103: pp. 564-576.

(32) McNulty PJ, Pearsall IS. Cavitation Inception in Pumps. ASME Journal of Fluids Engineering. 1982; Vol. 104: pp. 99-104.

(33) Robinette DL, Schweitzer JM, Maddock DG, Anderson CL, Blough JR, Johnson MA. Predicting the Onset of Cavitation in Automotive Torque Converters - Part II: A Generalized Model. International Journal of Rotating Machinery. 2008; Article ID 312753.

(34) Robinette DL. Detecting and Predicting the onset of Cavitation in Automotive Torque Converters. PhD Dissertation, Michigan Technological University. Ann Arbor: ProQuest/UMI. 2007.

(35) Kowalski D, Anderson CL, Blough JR. Cavitation Detection in Automotive Torque Converters Using Nearfield Acoustical Measurements. SAE-NVH. 5/16/2005; 2005-01-2516.

(36) Mekkes J, Anderson CL, Narain A. Static Pressure Measurements on the Nose of a Torque Converter Stator during Cavitation. ISROMAC. 2004.

(37) Robinette DL, Schweitzer JM, Maddock DG, Anderson CL, Blough JR, Johnson MA. Predicting the Onset of Cavitation in Automotive Torque Converters - Part II: A Generalized Model. International Journal of Rotating Machinery. 2008; Article ID 312753.

(38) Dong Y, Lakshminarayana B. Rotating Probe Measurements of the Pump Passage Flow Field in an Automotive Torque Converter. ASME J. of Fluids Eng.. 2001; Volume 123: pp. 81-91. 
(39) Madsen JI, Shyy W, Haftka RT. Response Surface Techniques for Diffuser Shape Optimization. AIAA Journal. 2000; Volume 38, No 9: pp. 1512-1518.

(40) Strasberg M, Taylor D. Propeller Cavitation Noise after 35 Years of Study. Noise and Fluids Engineering. 1977.

(41) Robinette DL, Anderson CL, Blough JR, Johnson MA, Maddock DG, Schweitzer JM. Characterizing the Effect of Automotive Torque Converter Design Parameters on the Onset of Cavitation at Stall. SAE-NVH. 5/15/2007; 2007-01-2231.

(42) Cakar O, Sanliturk KY. Elimination of Transducer Mass Loading Effects from Frequency Response Functions. Science Direct Mechanical Systems and Signal Processing. 2005; Volume 19: pp. 87-104.

(43) Heylen W, Lammens S, Sas P. Excitation Considerations. Modal Analysis Theory and Testing, Katholieke Universiteit Leuven. 1997.

(44) Homm, Ehrlich J, Peine H, Wiesner H. Experimental and Numerical Investigation of a Complex Submerged Structure, Part I: Modal Analysis. Acta Acustica united with Acustica. 2003; Volume 89: pp. 61-70.

(45) Robinette DL. Detecting and Predicting the onset of Cavitation in Automotive Torque Converters. PhD Dissertation, Michigan Technological University. Ann Arbor: ProQuest/UMI. 2007.

(46) Kowalski D, Anderson CL, Blough JR. Cavitation Detection in Automotive Torque Converters Using Nearfield Acoustical Measurements. SAE-NVH. 5/16/2005; 2005-01-2516.

(47) La Rosa Siqueira C. Wikipedia. File:Vortext-street-animation.gif. 2005. http://en.wikipedia.org/wiki/File:Vortex-street-animation.gif.

(48) Blevins R. Flow Induced Vibration. Krieger Publishing Company. 2001.

(49) Noctua Fan Company. Nine Blade Design with Vortex-Control Notches. 2012; http://www.noctua.at/main.php?show=nine_blade_design\&lng=en.

(50) Walber C, Blough JR, Anderson CL, Johnson MA. Measuring and Comparing Frequency Response Functions of Torque Converter Turbines Submerged In Transmission Fluid. SAE-NVH. 5/17/2011; 2011-01-1662.

(51) Sweger P, Anderson CL, Blough JR. Measurements of Strain on $310 \mathrm{Mm}$ Converter Turbine Blade. International Journal of Rotating Machinery. 2004; Volume 10, No 1: pp. 55-63. 
(52) Mekkes J, Anderson CL, Narain A. Static Pressure Measurements and Cavitation Signatures on the Nose of a Torque Convertor's Stator Blade. ISROMAC. 2004.

(53) Robinette DL, Schweitzer JM, Maddock DG, Anderson CL, Blough JR, Johnson MA. Predicting the Onset of Cavitation in Automotive Torque Converters-Part I: Designs with Geometric Similitude. International Journal of Rotating Machinery. 2008; Article ID 803940. 


\section{Appendix A: Matlab Code}

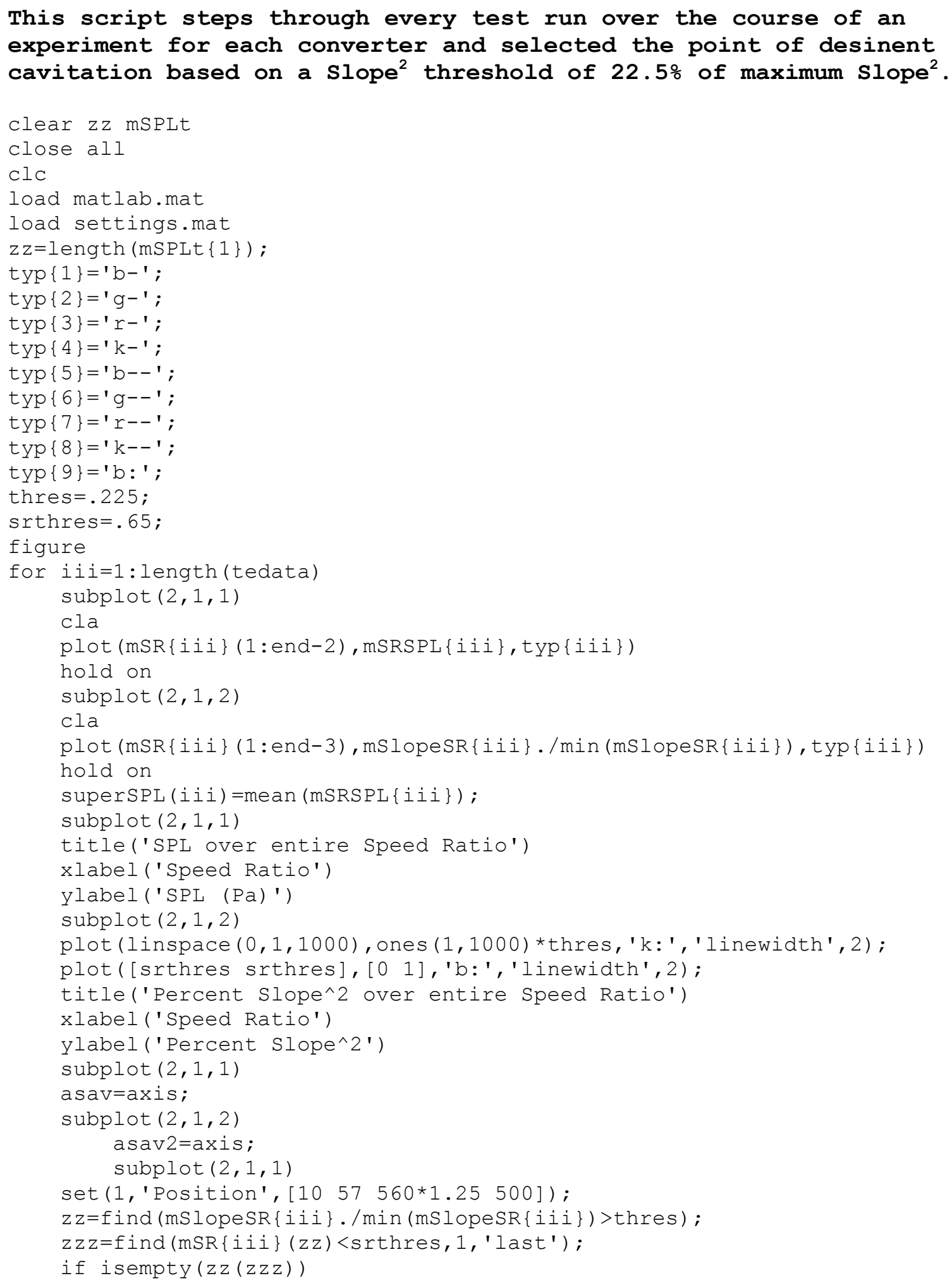




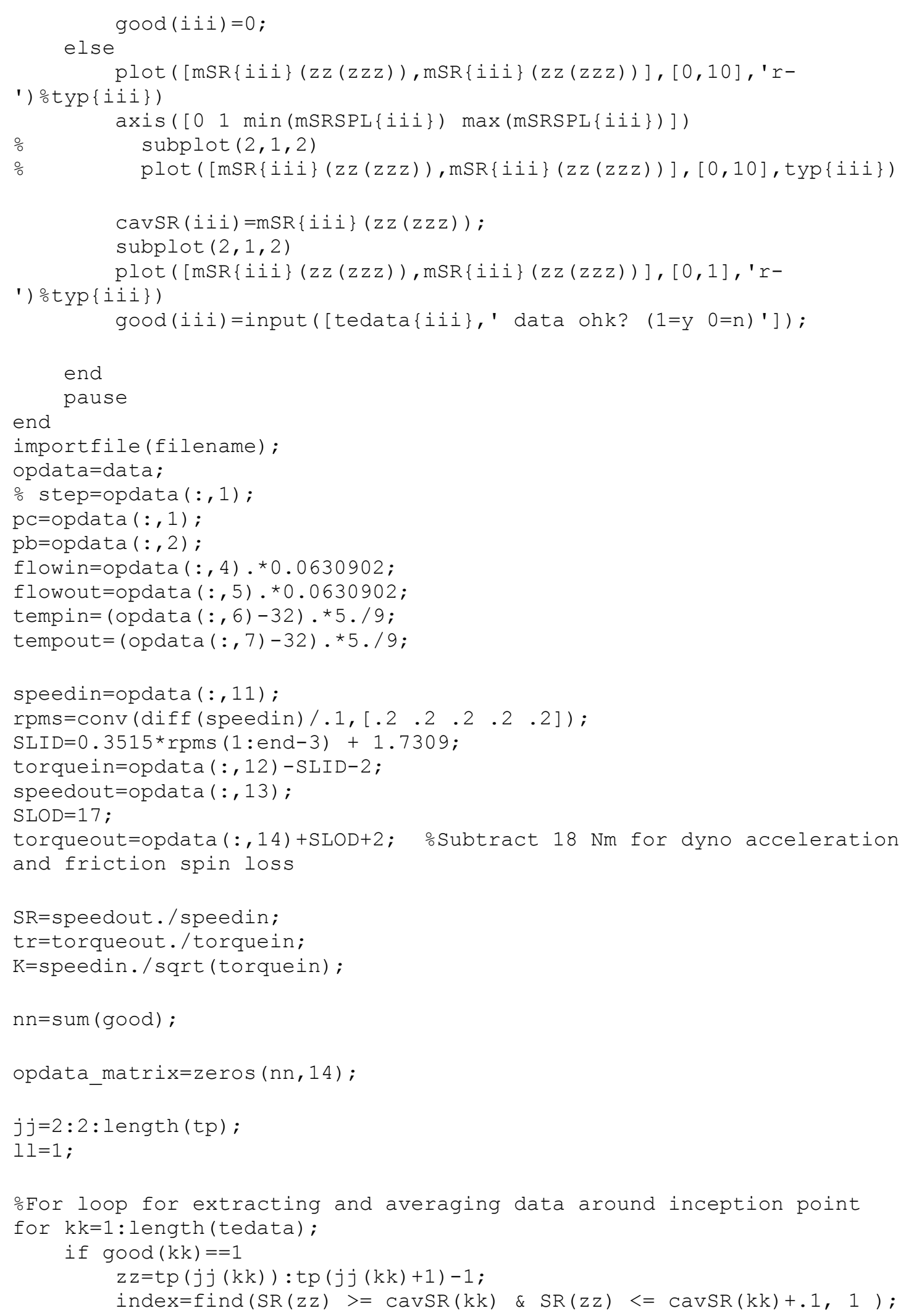




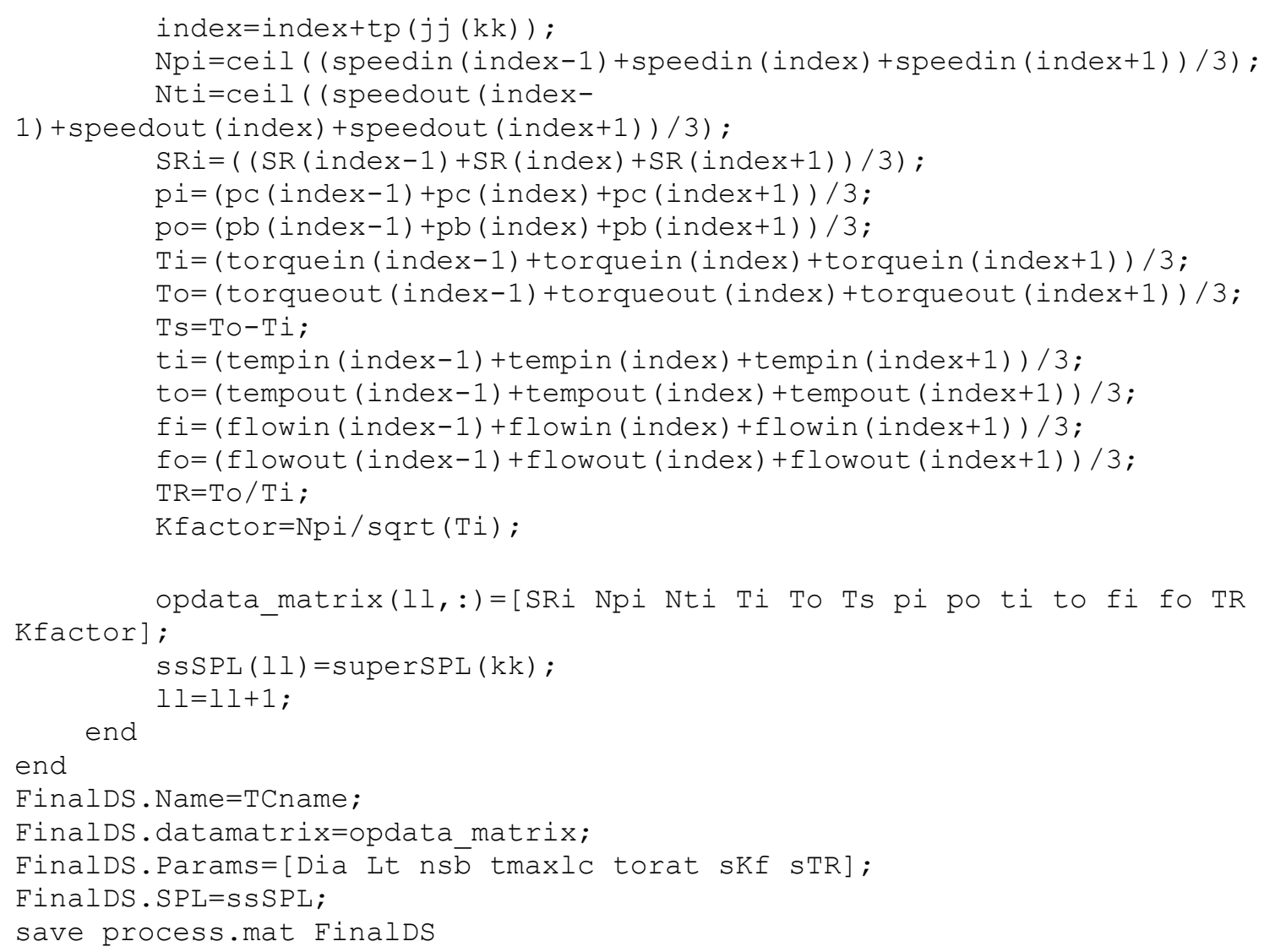




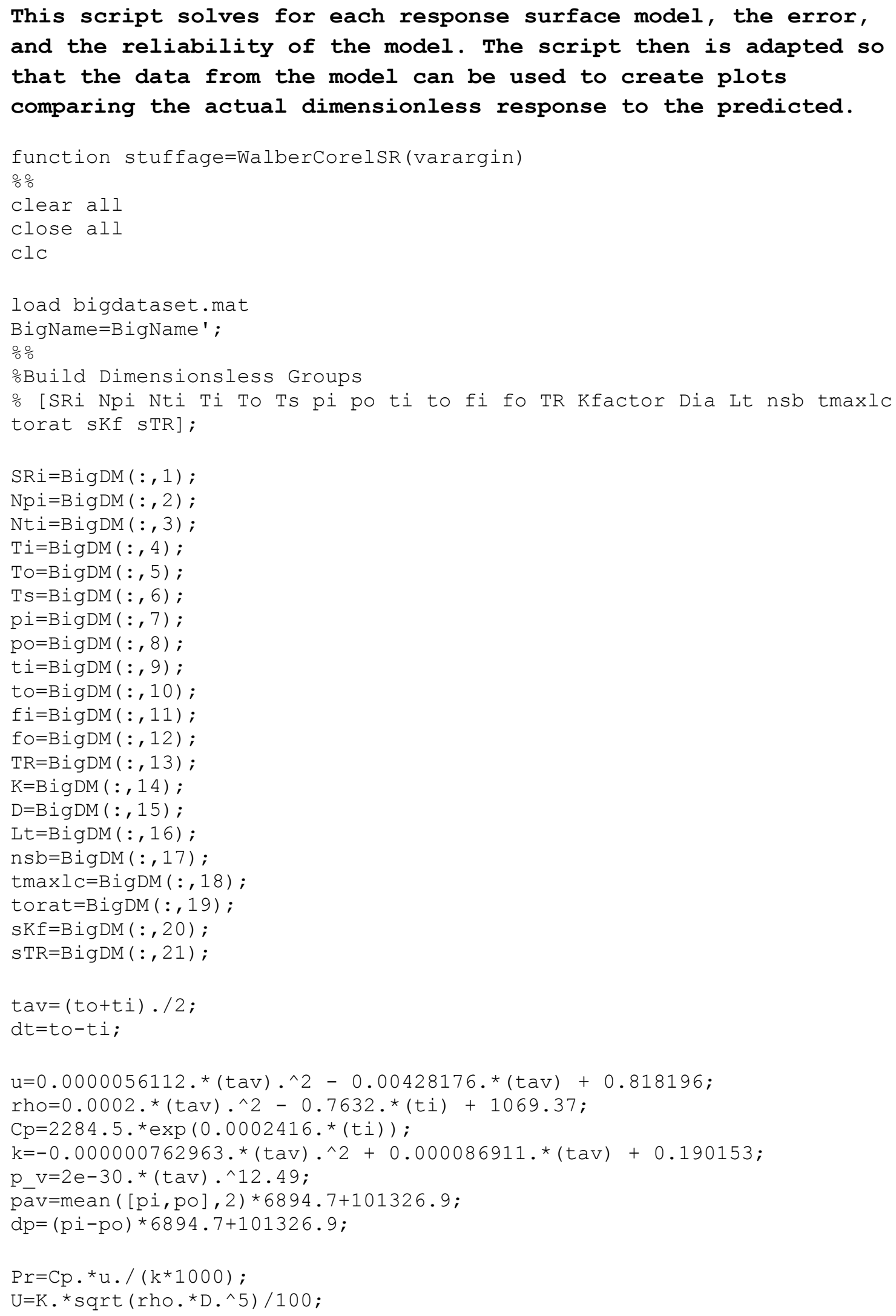




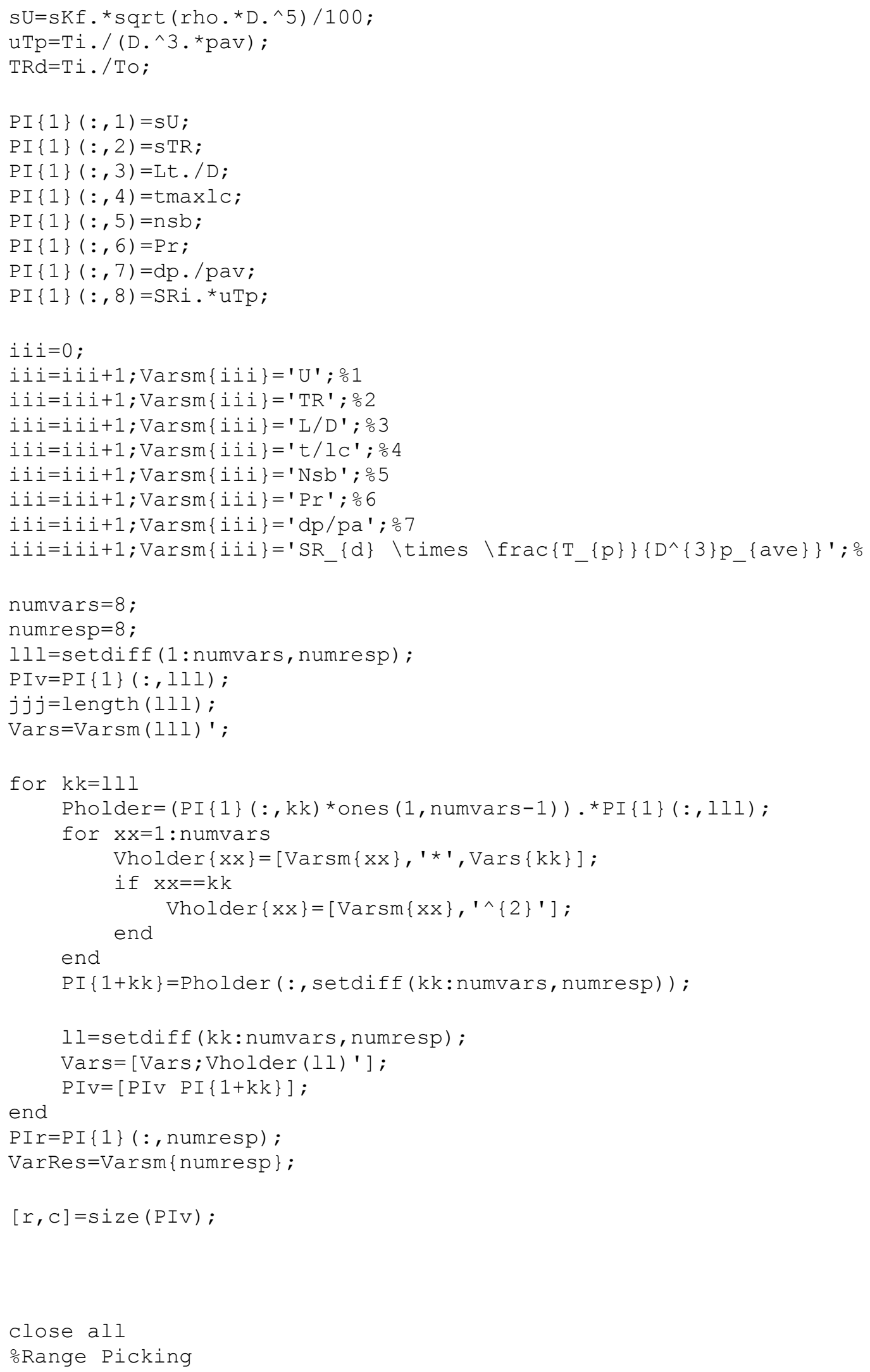




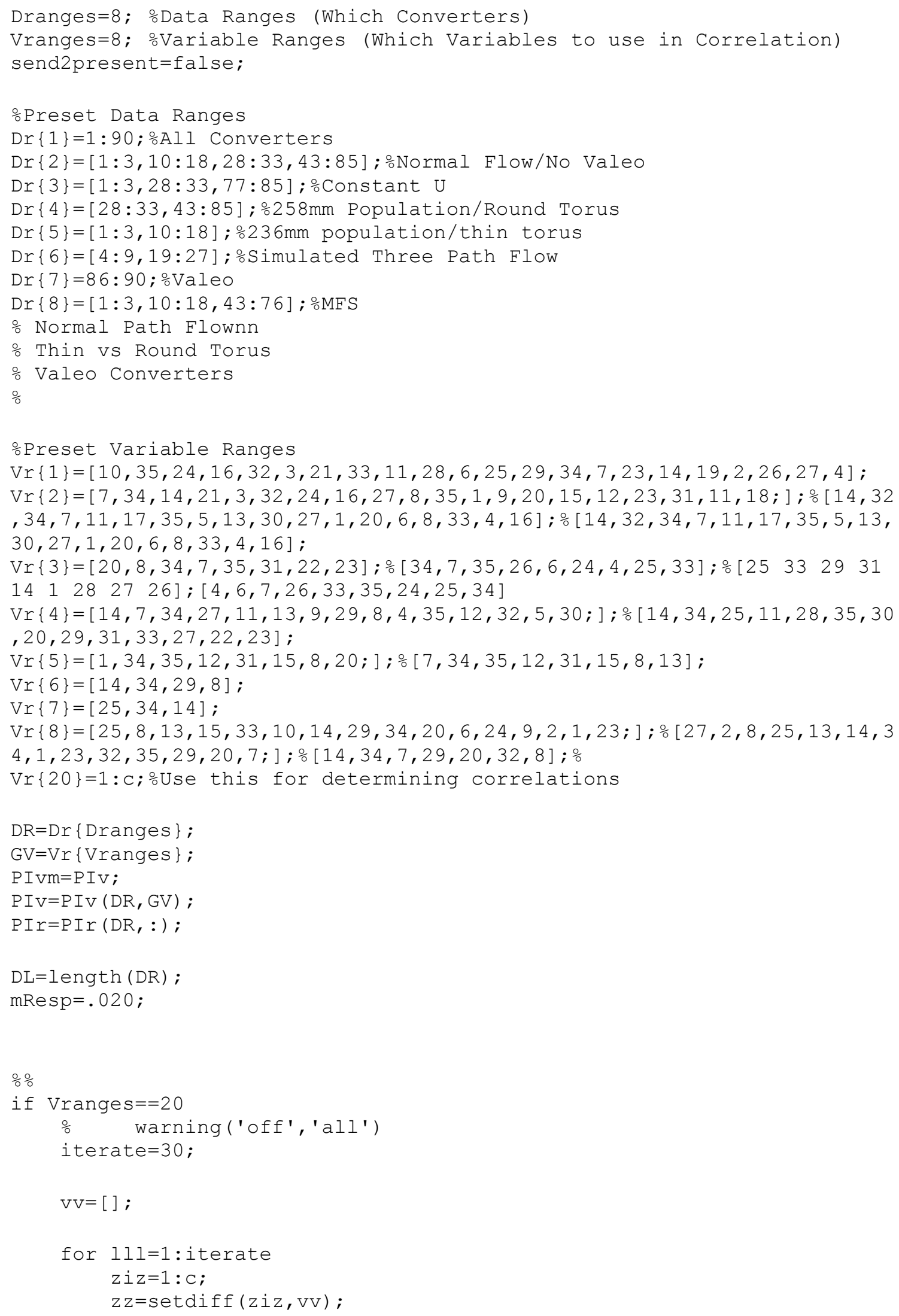




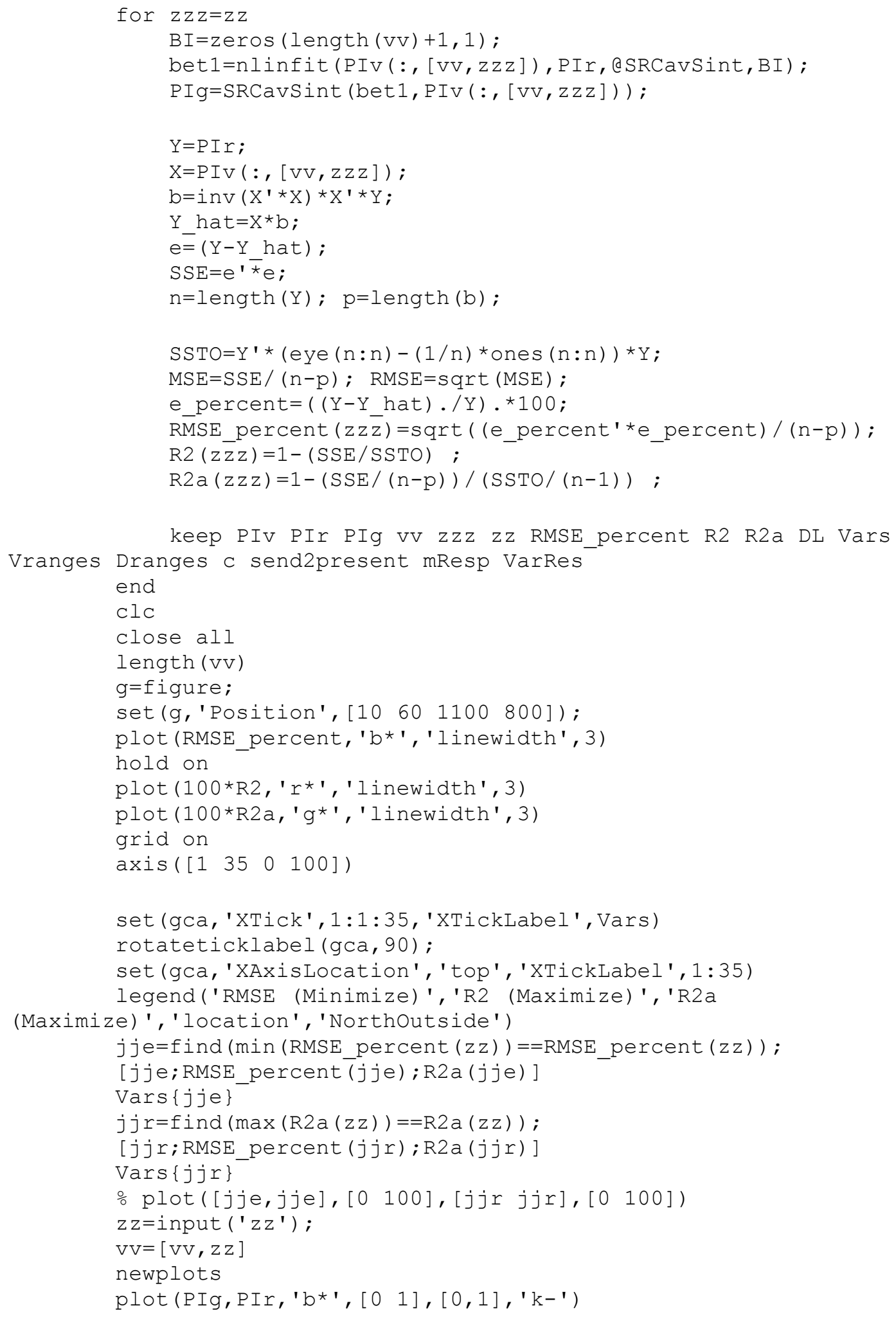




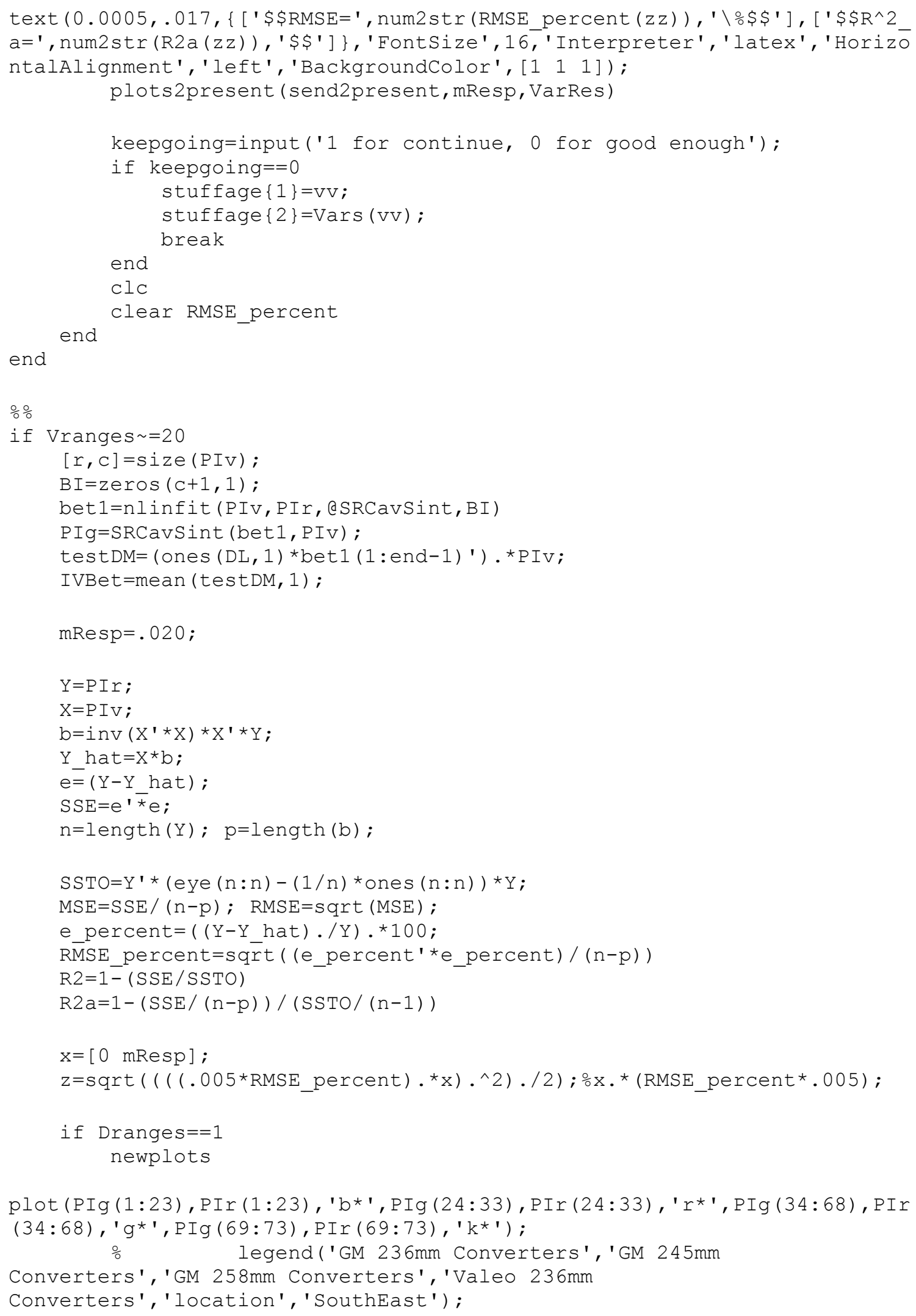


legend('236mm Diameter Converters','245mm Diameter

Converters','258mm Diameter Converters', 'Valeo $236 \mathrm{~mm}$

Converters', 'location', 'SouthEast');

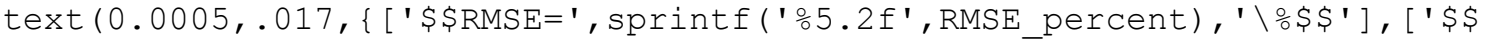
R^2 a=', sprintf('⒌3f', R2a), '\$\$']\},' FontSize', 16,' Interpreter', 'latex'

,'HorizontalAlignment', 'left', 'BackgroundColor', [ [ $\left.\left.1 \begin{array}{ll}1 & 1\end{array}\right]\right)$;

end plots2present (send2present, mResp, VarRes)

if Dranges $==2$

newplots

plot (PIg (1:12), PIr (1:12), 'b*' , PIg(13:18), PIr (13:18), 'r*', PIg (19:61), PIr $(19: 61), ' g * ')$;

legend('236mm Diameter Converters','245mm Diameter

Converters','258mm Diameter Converters', 'location', 'SouthEast');

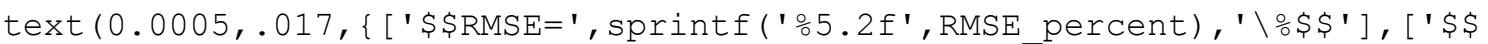
R^2_a=', sprintf('⒌3f', R2a),'\$\$']\},' FontSize', 16,' Interpreter', 'latex'

, 'HorizontalAlignment', 'left', 'BackgroundColor' , [l $\left.\left.\begin{array}{lll}1 & 1 & 1\end{array}\right]\right)$;

plots2present ( send2present, mResp, VarRes)

newplots

plot (PIg (1:3), PIr (1:3), 'b*', PIg ( [4:18,52:61]), PIr ([4:18,52:61]), 'r*', PI $\left.g(19: 35), \operatorname{PIr}(19: 35),{ }^{*}{ }^{*} ', \operatorname{PIg}(36: 51), \operatorname{PIr}(36: 51), \mathrm{k}^{*} \mathbf{\prime}^{\prime}\right)$; legend('29 Stator Blades','21 Stator Blades','17 Stator

Blades','15 Stator Blades','location', 'SouthEast');

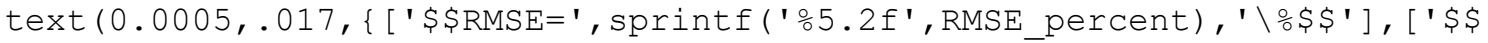
R^2 a=', sprintf('⒌ 5 . ' ', R2a), '\$\$']\}, 'FontSize', 16,' Interpreter', 'latex'

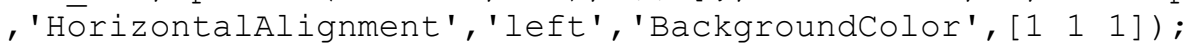

plots 2 present (send2present, mResp, VarRes)

for $\mathrm{pp}=1:$ length (Vr $\{$ Dranges $\})$

$\mathrm{PIV}=\mathrm{PIVm}(\mathrm{DR}, \mathrm{GV}(1: \mathrm{pp}))$;

cond (PIV)

$[r, c]=\operatorname{size}(P I v)$;

$\mathrm{BI}=\operatorname{zeros}(\mathrm{C}+1,1)$;

bet $1=n l i n f i t(P I V, P I r$, aSRCavSint,BI) ;

PIg=SRCavSint (bet $1, \mathrm{PIV})$;

test $\mathrm{DM}=($ ones $(\mathrm{DL}, 1)$ *bet1 $(1$ : end -1$)$ ' $) . * \mathrm{PIV}$;

IVBet=mean (testDM, 1);

$\mathrm{mResp}=.020$;

$\mathrm{Y}=\mathrm{PIr} ;$

$\mathrm{X}=\mathrm{PIV}$;

$\mathrm{b}=\operatorname{inv}\left(\mathrm{X}^{\prime} \star \mathrm{X}\right) * \mathrm{X}^{\prime} * \mathrm{Y}$;

$Y$ hat $=X * b$;

$\mathrm{e}=(\mathrm{Y}-\mathrm{Y}$ hat $)$;

$\mathrm{SSE}=\mathrm{e}^{\prime \bar{\star}} \mathrm{e}$;

$\mathrm{n}=$ length (Y); $\mathrm{p}=$ length (b) ; 


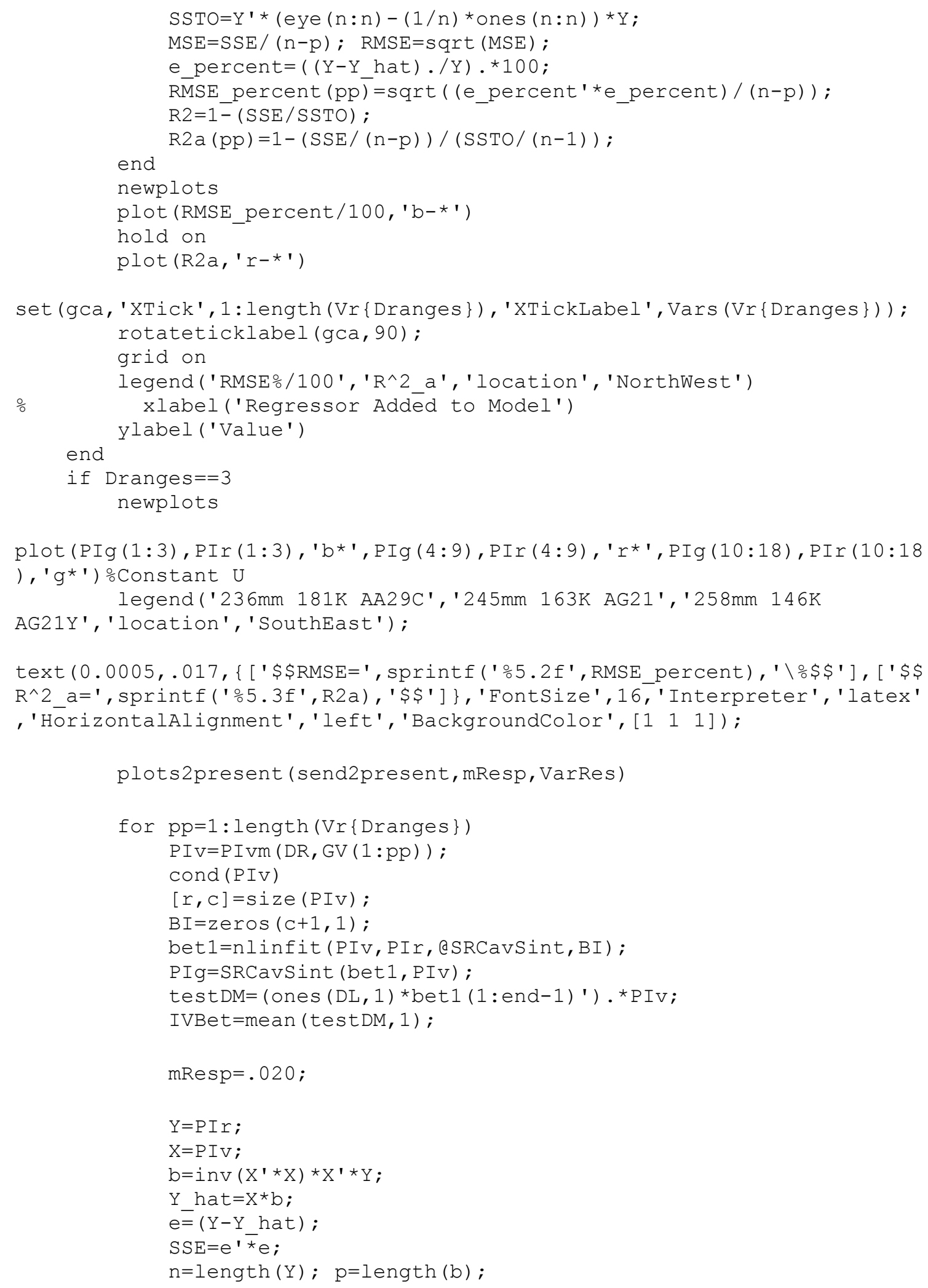




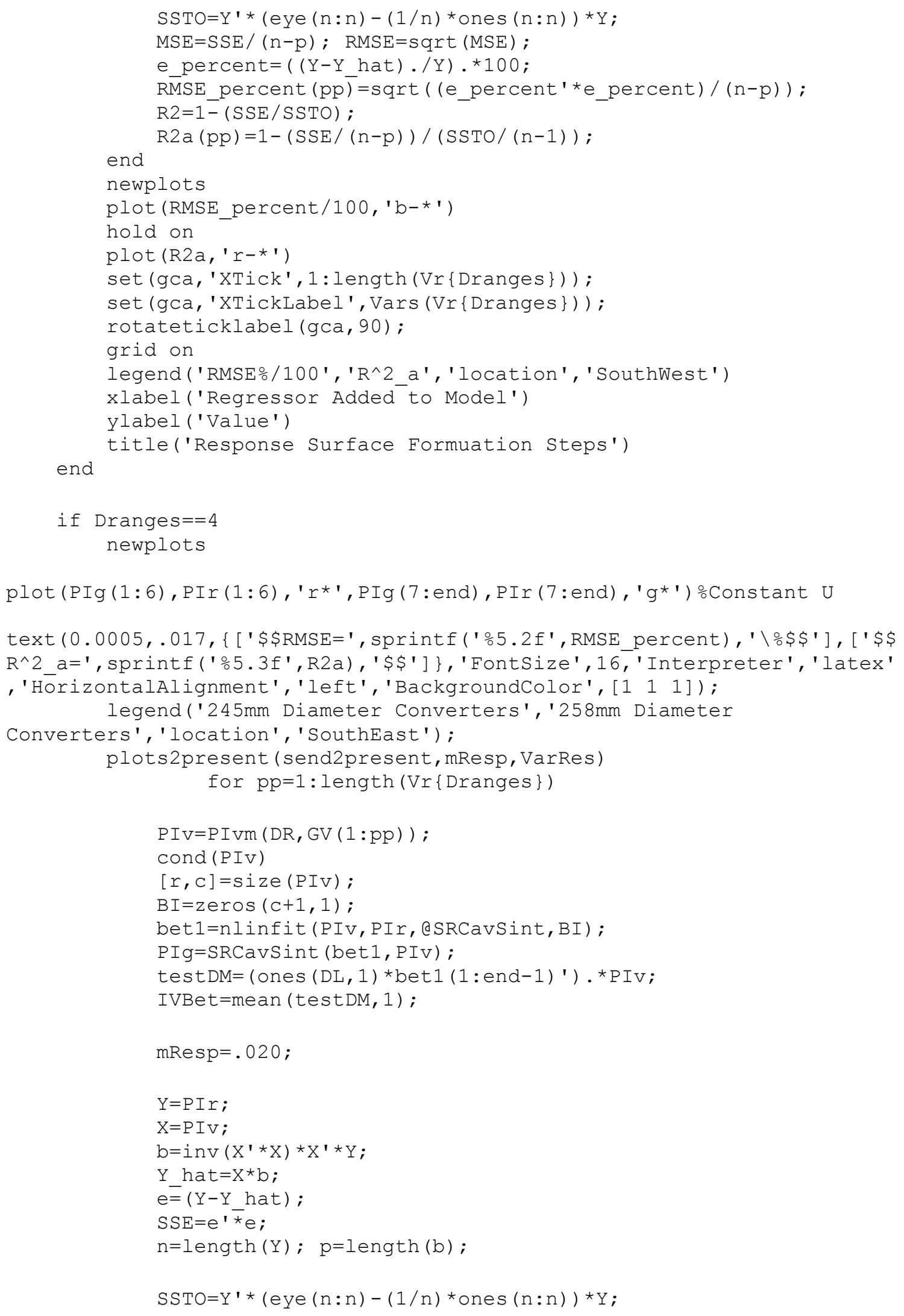




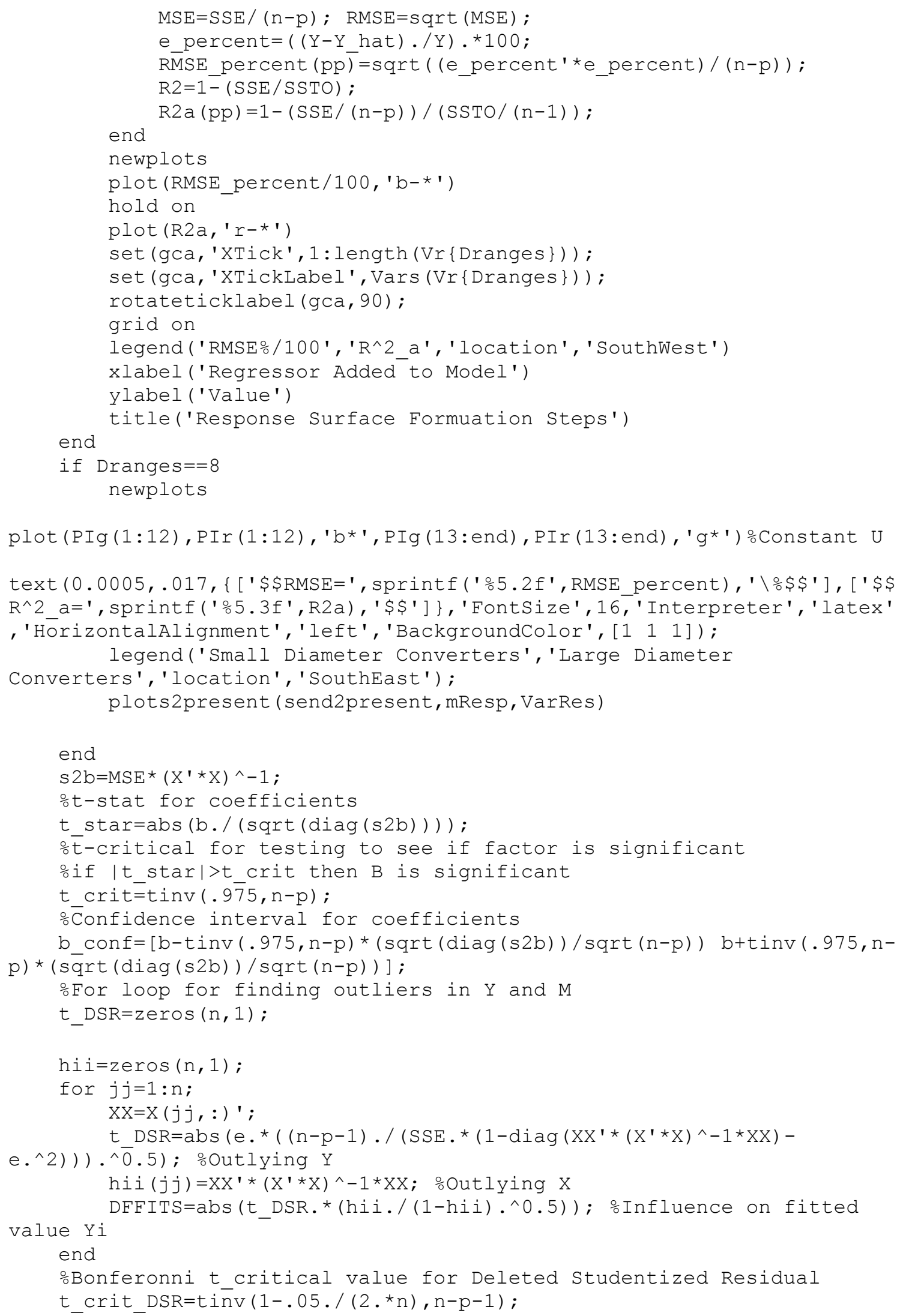




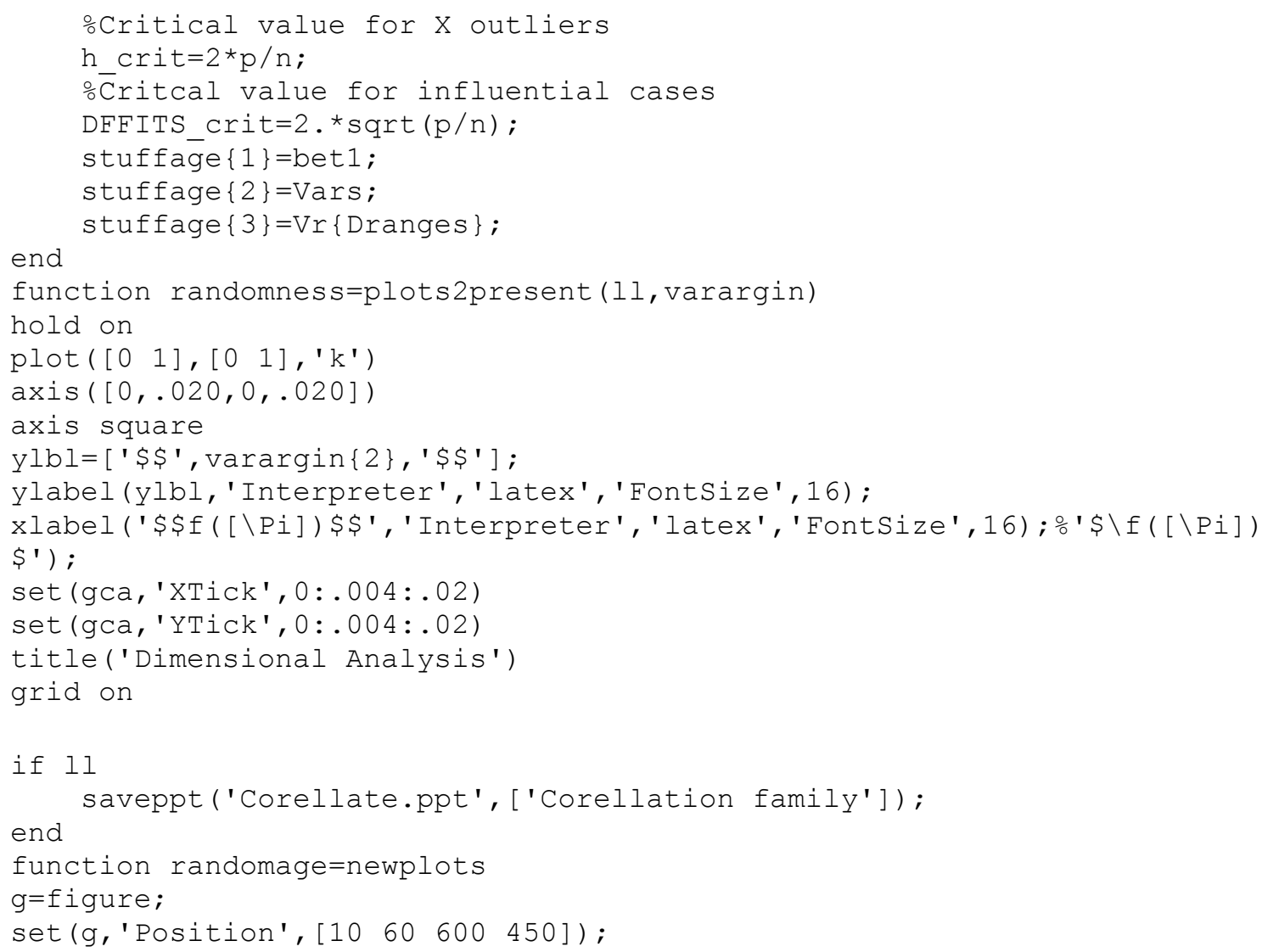




\section{These scripts are the acquisition software for the VXI data acquisition hardware. The computer running this needs Agilent IO drivers and the DAQ Toolbox for Matlab installed.}

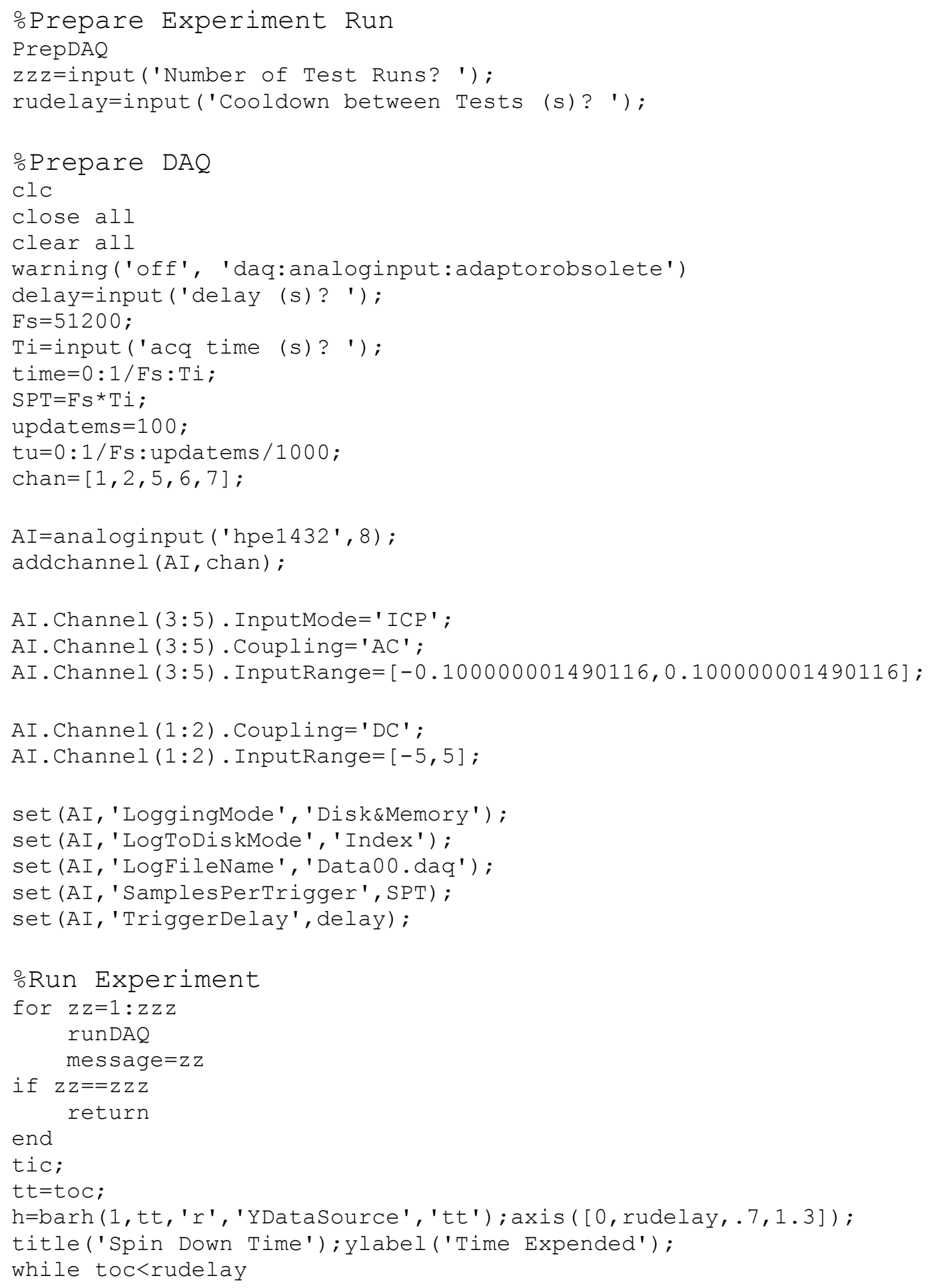




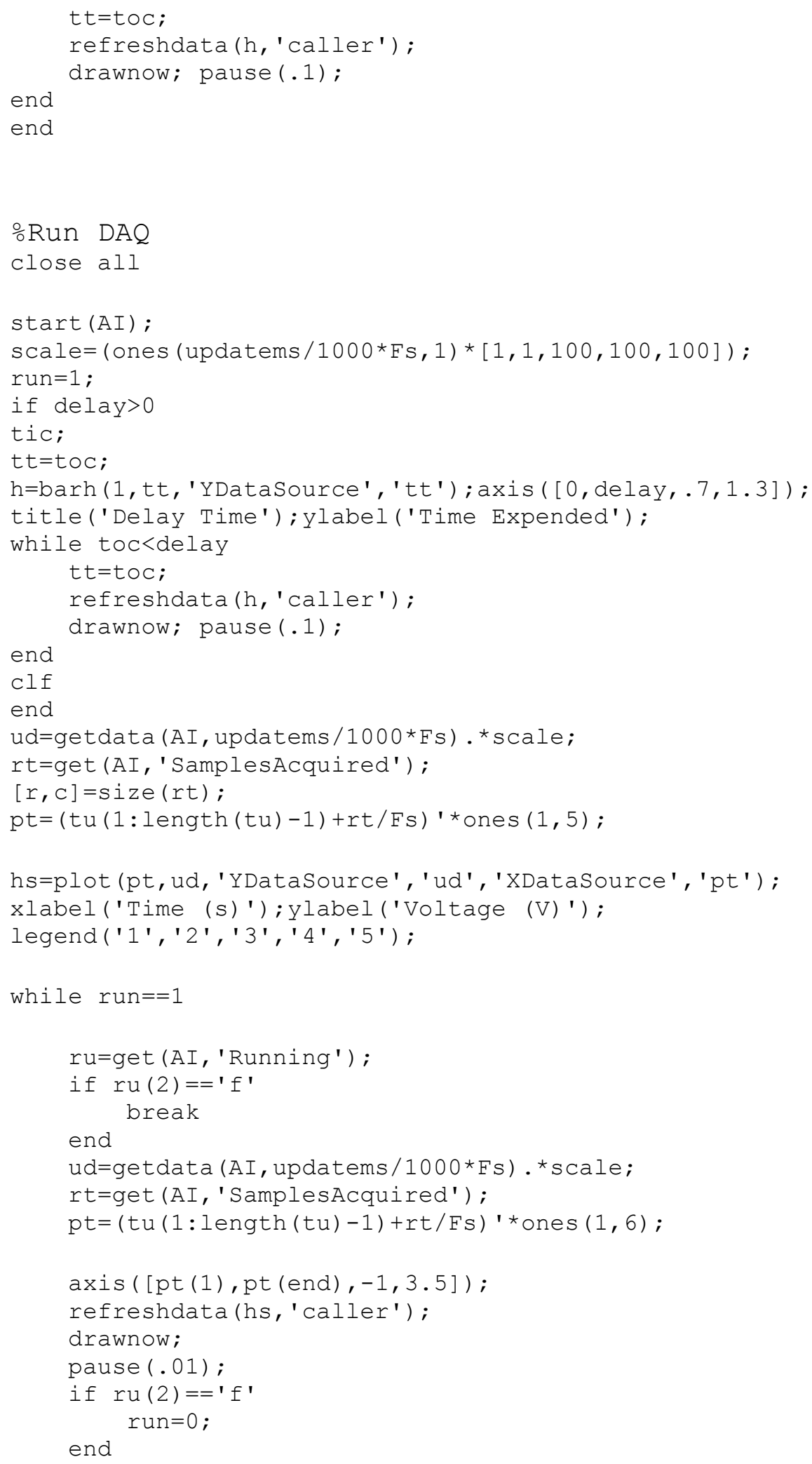




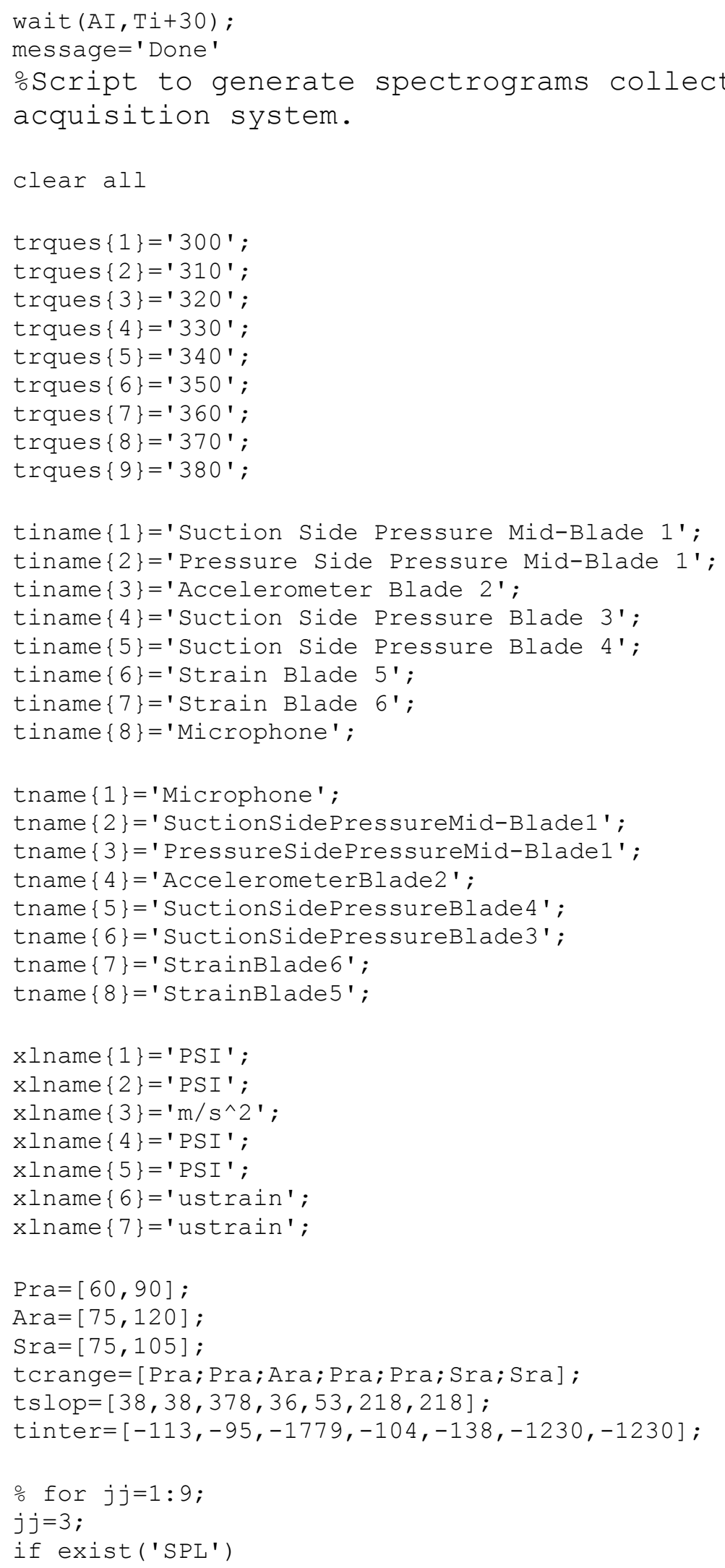




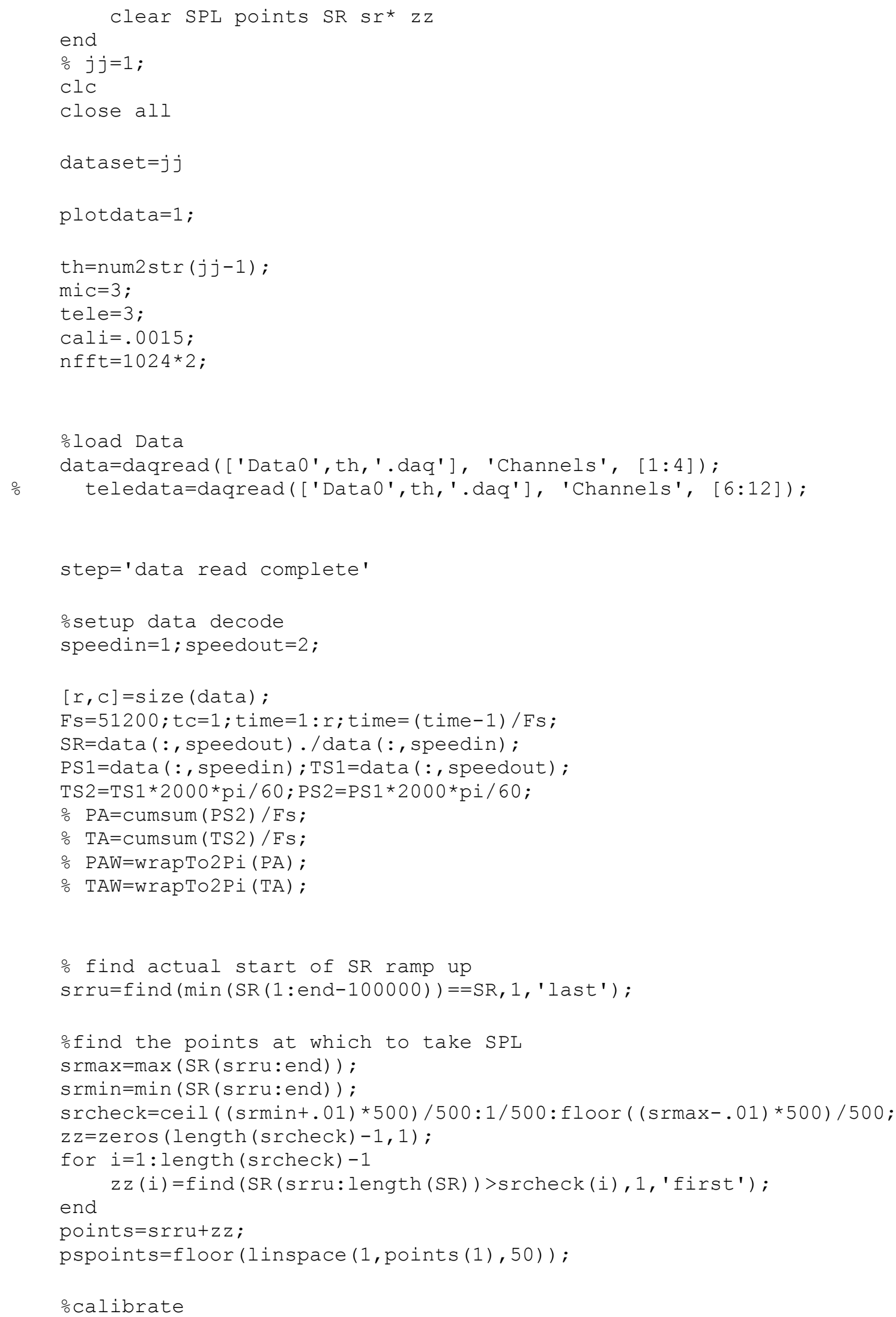




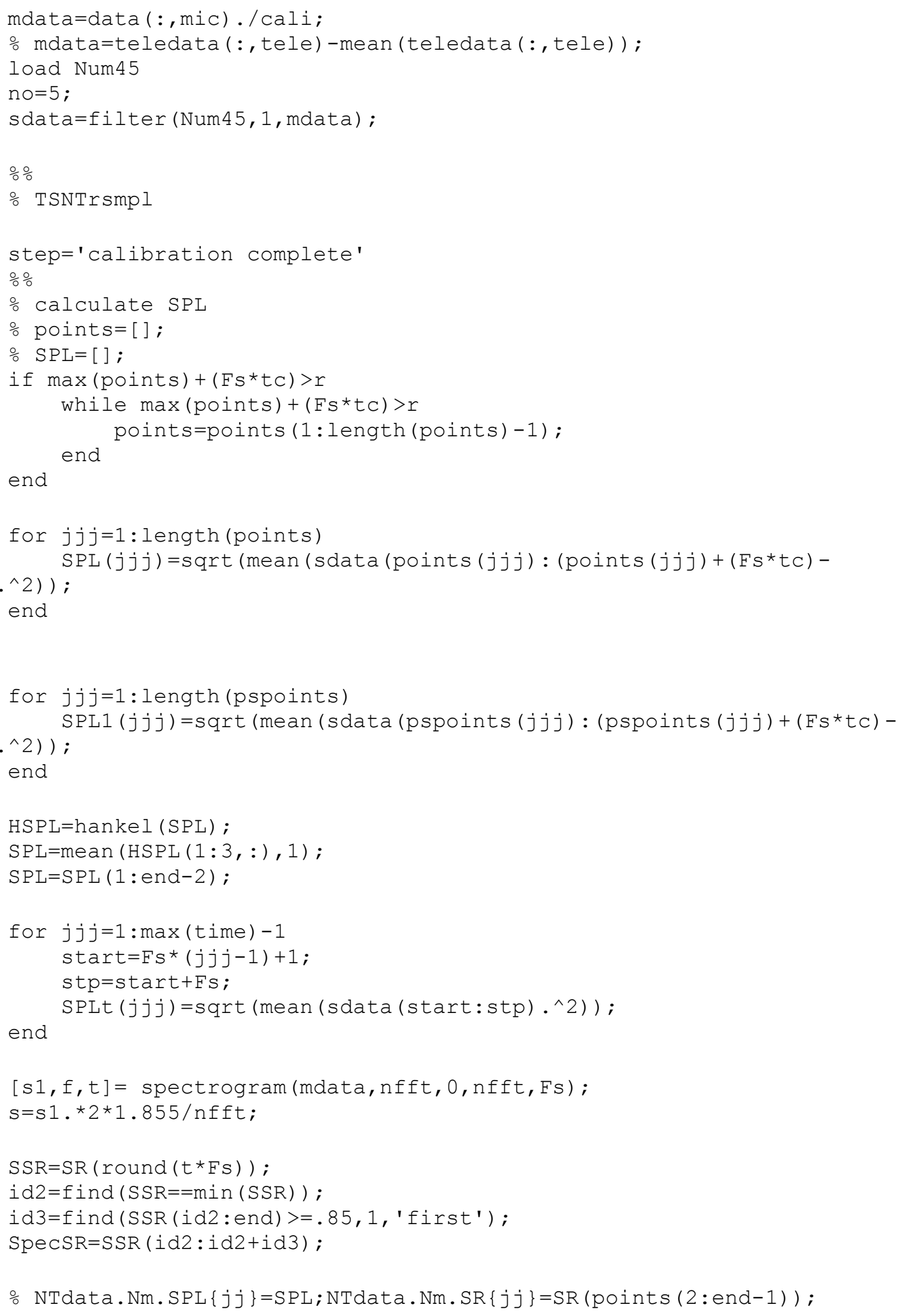




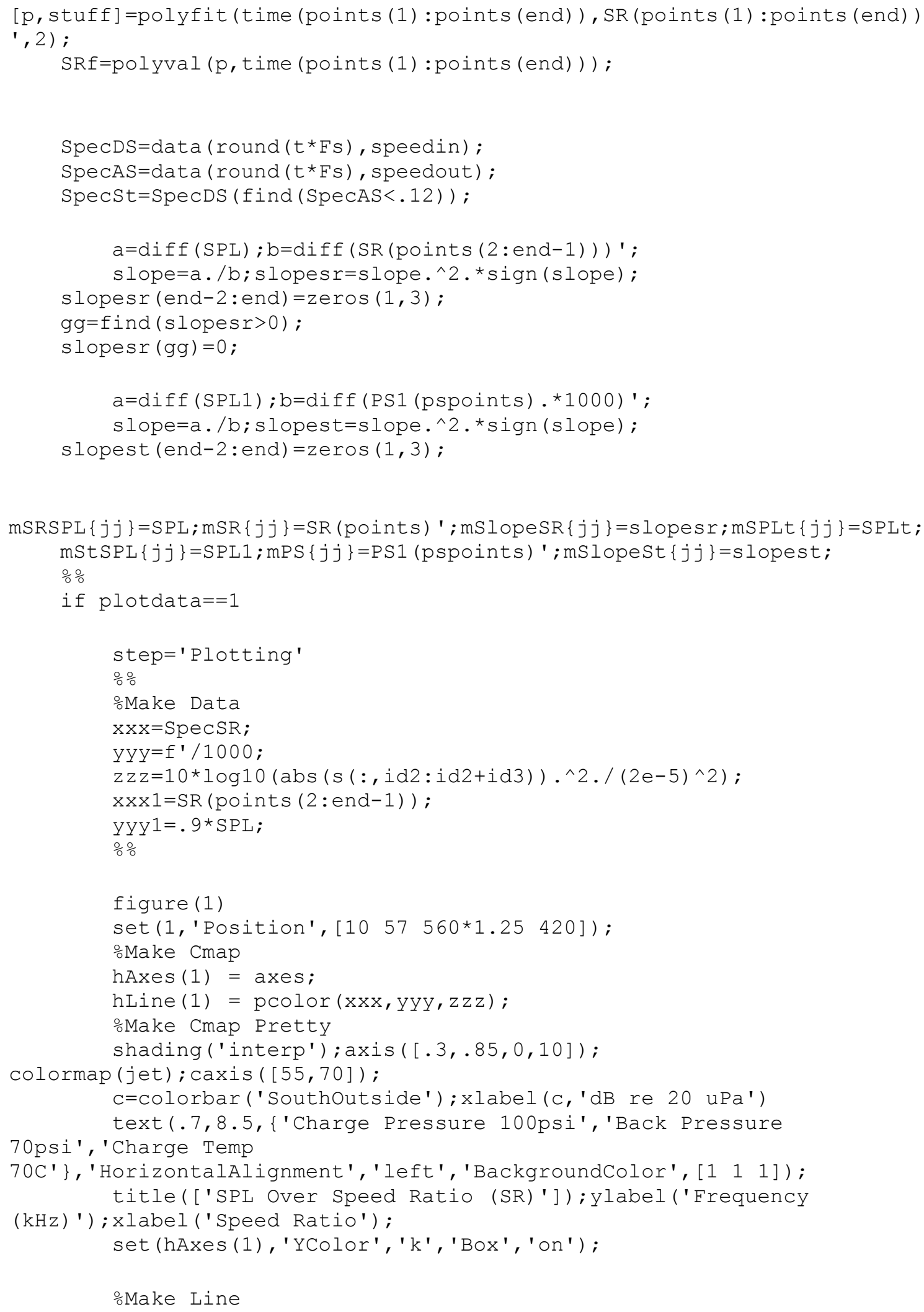




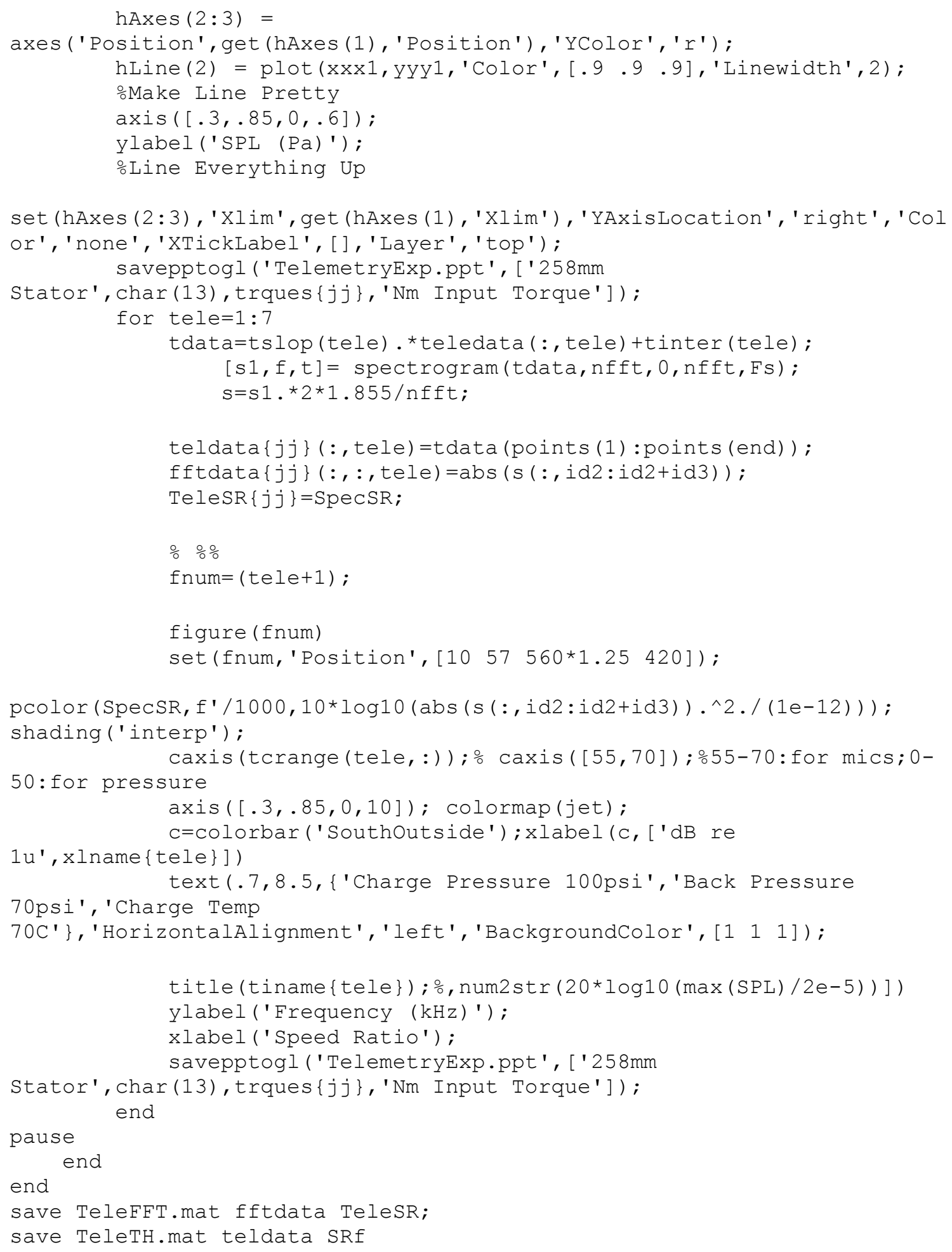




\section{Appendix B: Submerged Turbine Modal Analysis}

Chapter 3 discussed the procedure for measuring frequency response functions of a torque converter turbine in dry air and in automatic transmission fluid (ATF). The results were discussed in Chapter 3 and again in Chapter 4. This appendix gives a more complete description of the testing and the analysis performed after.

Both known noisy and quiet turbines were tested in an effort to determine if by dynamically testing alone, that is, measuring the frequency response can be used to predict whether or not a turbine would be noisy. Figure B.1 shows a plot of the autopower response at the trailing edge of one blade of five different turbine constructions. The excitation for each of these was a ball bearing dropped from a constant height onto the trailing edge of a turbine blade. This excitation method is further outlined by Walber [6]. Since the noise is known to be affected greatly by modifications to the trailing edge, only driving point measurements were taken at the trailing edge of turbine blades.

There were some differences between all five of the turbines tested, however nothing definitive further stood out to differentiate the two turbines that made noise from the ones that did not. The main natural frequencies of the two noisy turbines, the unnotched turbines, were separated by about $1000 \mathrm{~Hz}$, with all of the quiet turbines, the machined from solid and two notched turbines, interspaced in between. 


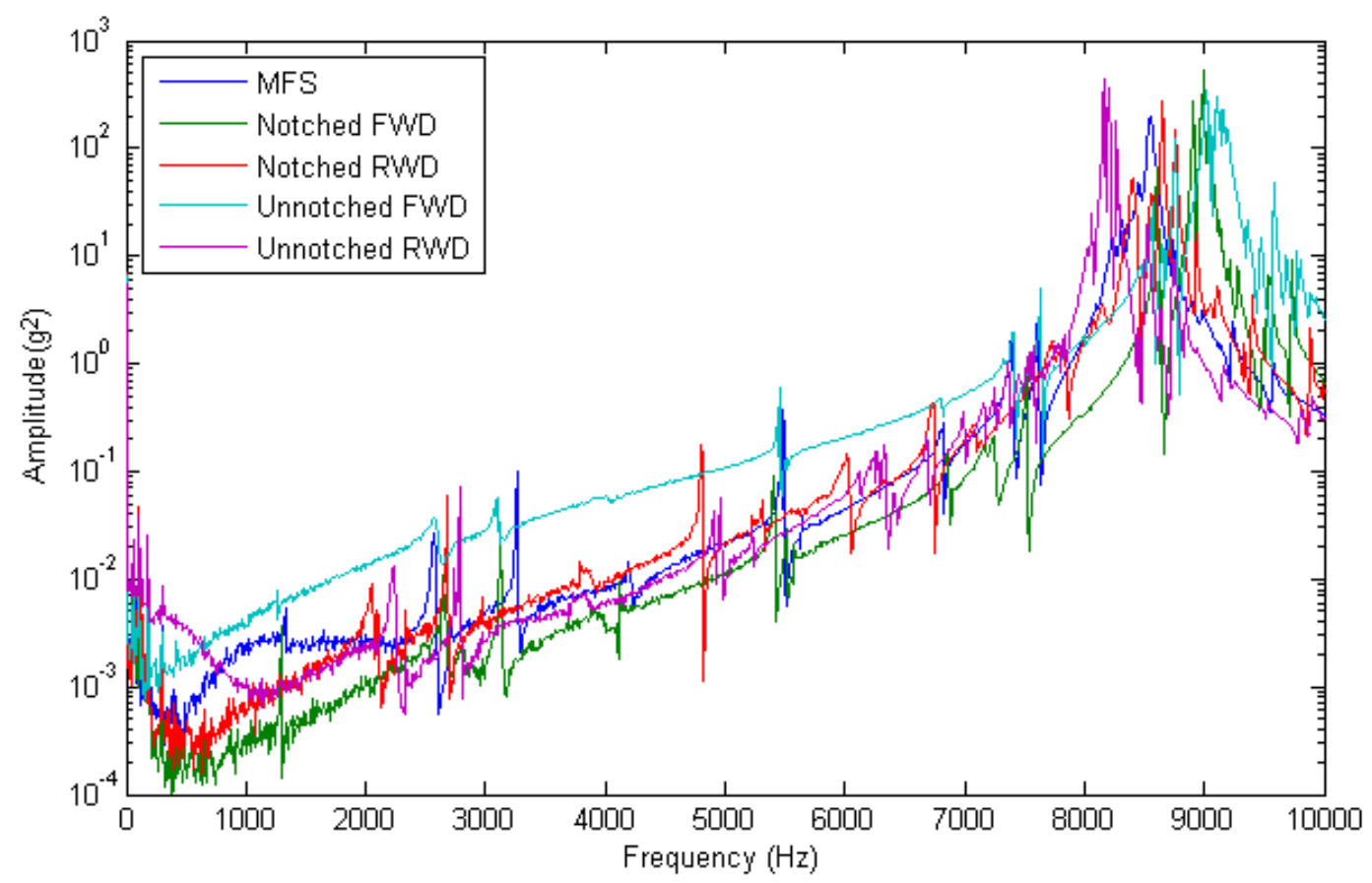

Figure B.1: Comparison of autopower response of turbines known to be noisy during operation (Both unnotched turbines) to those that were known to be quiet during operation (MFS and both notched turbines) in air.

Modal testing was performed to determine if blade to blade variation on a specific turbine was as great as the variation from turbine to turbine. A roving impact method of excitation was used with three accelerometer references for the frequency response functions. Reference data was taken at the center of one of the turbine trailing edge blades, on the hub of the turbine, and on the outer shell of the turbine. Response measurements were collected using an impact hammer at three points along four different blades, eight points around the hub, 32 points around the surface of the shell of the turbines, and one point that corresponded to the driving point blade. Some of this data is shown in Figure B.2 and Figure B.3. 


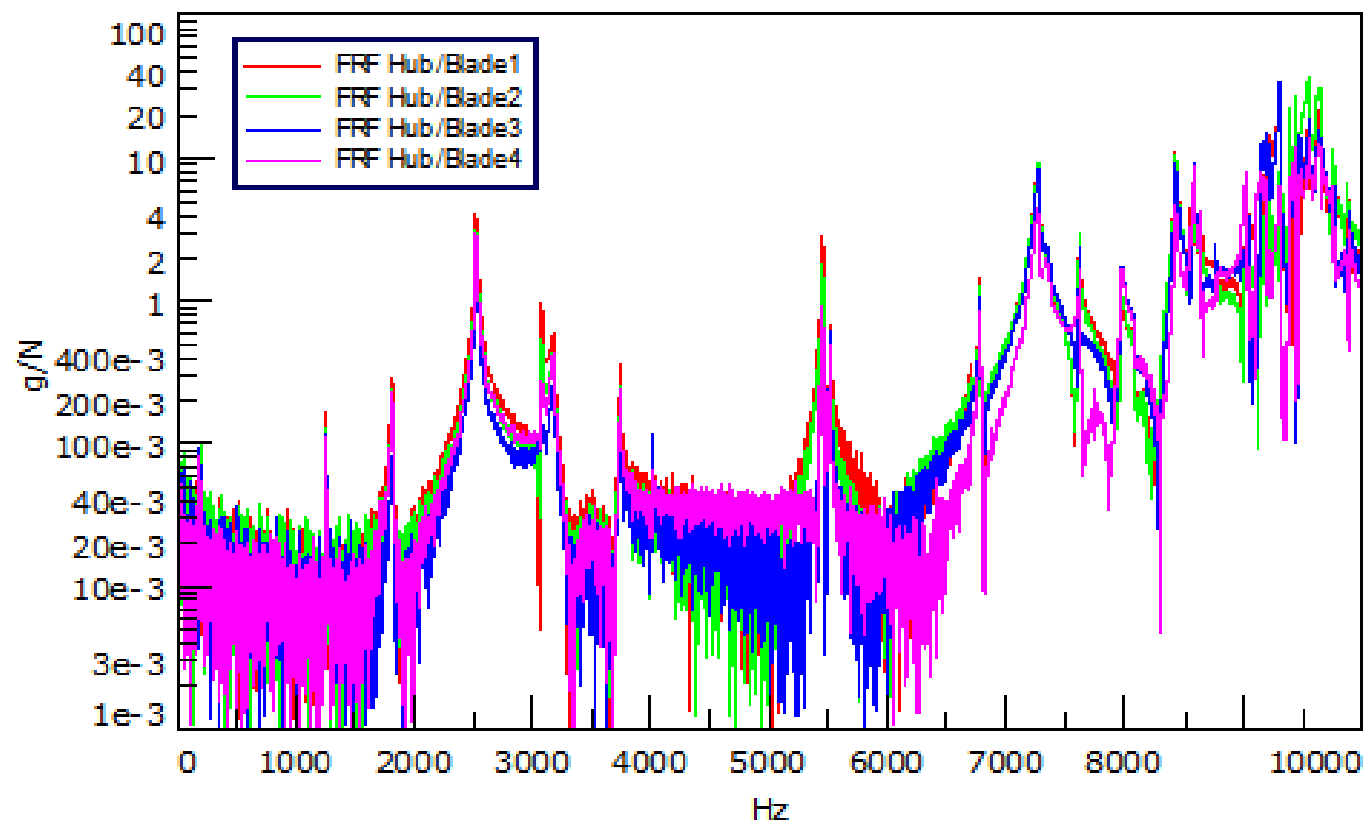

Figure B.2: Comparison of frequency response functions of a reference point on the hub of the unnotched turbine of a noisy turbine with four trailing edge blades tested in air.

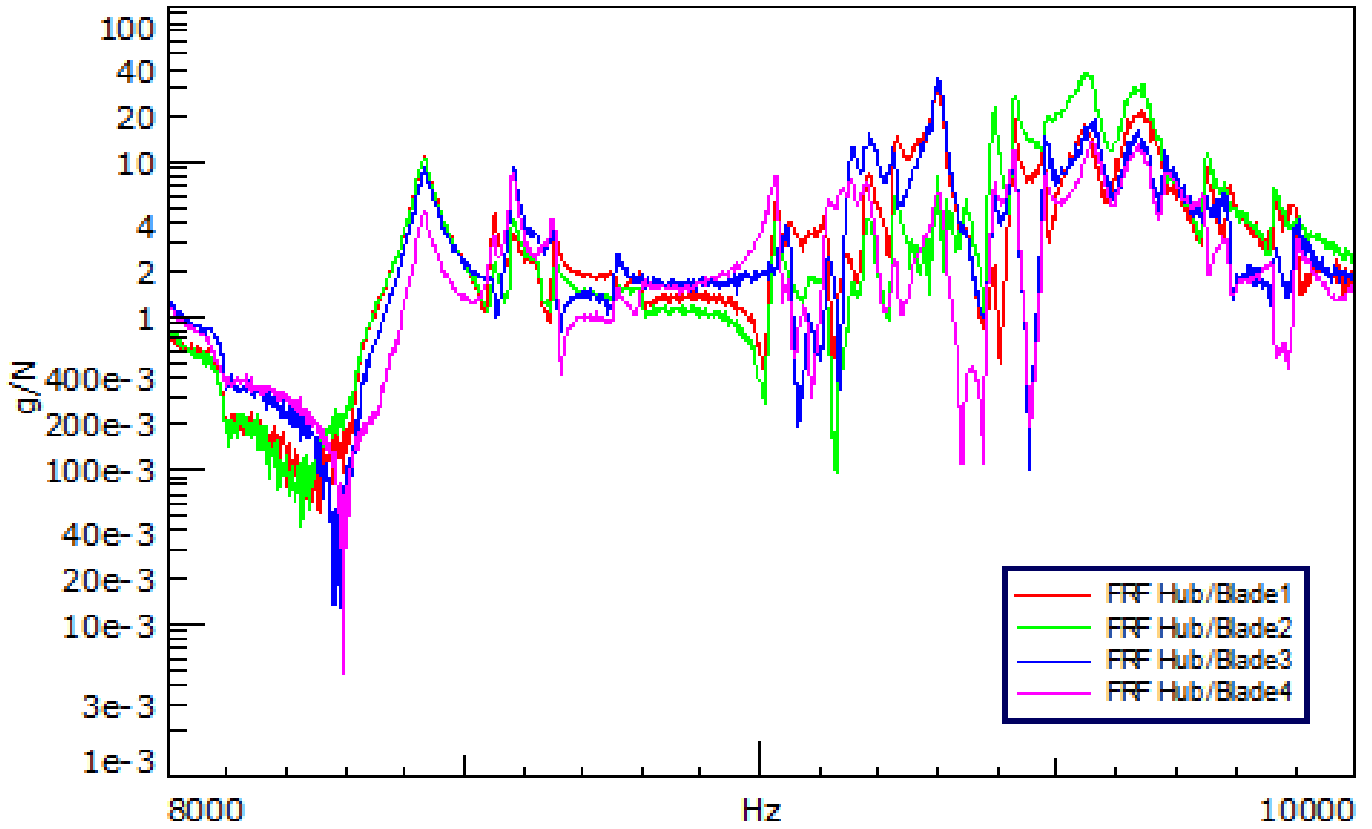

Figure B.3: Comparison of frequency response functions of a reference point on the hub of the unnotched turbine of a noisy turbine with four trailing edge blades zoomed in to the natural frequencies thought to be associated with the trailing edge blades tested in air. 
Figure B.2 shows that each blade has a very small variation with respect to the others when the hub is used as a frequency response reference point and looking at the entire frequency range. However, Figure B.3 shows what was believed to be the individual natural frequencies associated with each turbine blade. Here it was much more evident that individual blade variation would make it difficult to determine whether or not a turbine was noisy based on an impact test. Another thing to note here is that there does not appear to be a natural frequency at $4 \mathrm{kHz}$, the frequency at which the turbine noise occurs. However, this data was taken in air, which is not the normal operating medium for the torque converter.

The vibration mode of the turbine at the frequency of the noise in the operating medium, ATF, became the next point of investigation. This was to learn not only if there was an actual mode of vibration at the frequency of interest, but also to learn the shape of the motion. The challenge in this is that the frequency response of a structure is different when it is in air from what it is in a more dense fluid, such as ATF. For this reason the turbine was tested for mode shapes both in and out of the transmission fluid. Only one turbine, an unnotched turbine, was tested both in and out of ATF since it was already determined that it could not easily be differentiated dynamically from a notched turbine or other quiet turbine. The same physical points on the structure were measured when the turbine was placed in ATF as when it was in air.

The data from the submerged testing is shown in Figure B.4. The physical points referenced on the turbine where the data was measured for Figure B.4 are identical to those measured in Figure B.2. The comparison between test results in air and in ATF shows that the natural frequencies of the turbine in ATF shift almost uniformly to lower frequencies, while the overall amplitude of the data remains about the same. This means that the addition of ATF to the turbine acts as mass loading with regards to vibration, while very little damping can be detected here. This shift in the natural frequencies placed a resonance right at $4 \mathrm{kHz}$ and a second resonance at around $4.3 \mathrm{kHz}$. Both of these can be seen in the peaks shown in Figure B.4. 


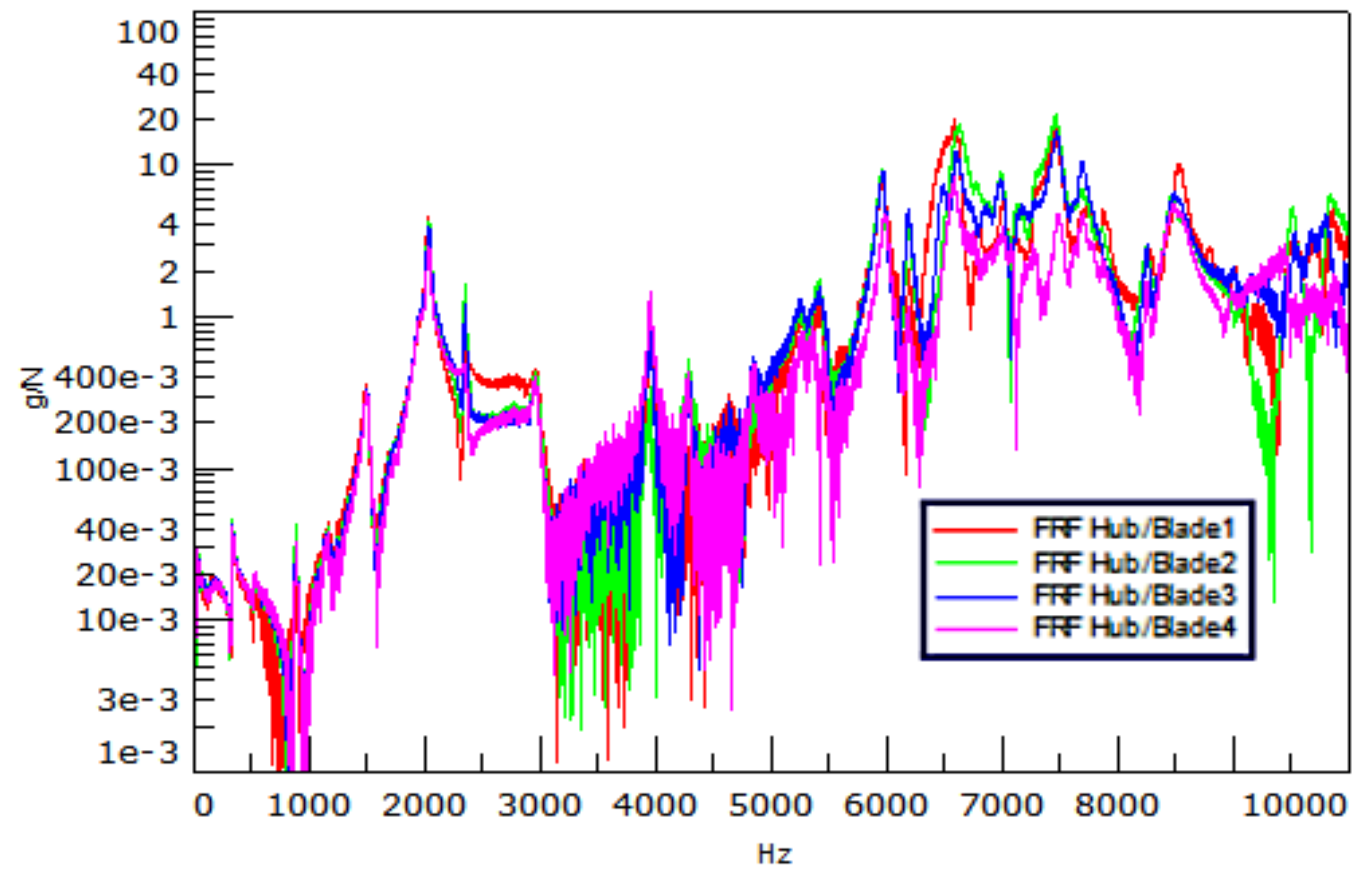

Figure B.4: Comparison of frequency response functions of a reference point on the hub of the unnotched turbine of a noisy turbine with four trailing edge blades tested in ATF.

All of the frequency response functions for each set of measurements were curve-fit, and solved for mode shapes and natural frequencies. Two sets of mode shapes were found to be of interest in the turbine submerged in ATF near $4 \mathrm{kHz}$. These modes are shown in Figure B.5 and Figure B.6. The modal assurance criteria (MAC) of each mode in air were calculated with reference to its corresponding mode in ATF. MAC is a measure of similarity between the two mode shapes, equivalent to the dot product of the vectors containing the amplitudes of the mode shapes. The goal was to show that each of these pairs of modes were very similar. 


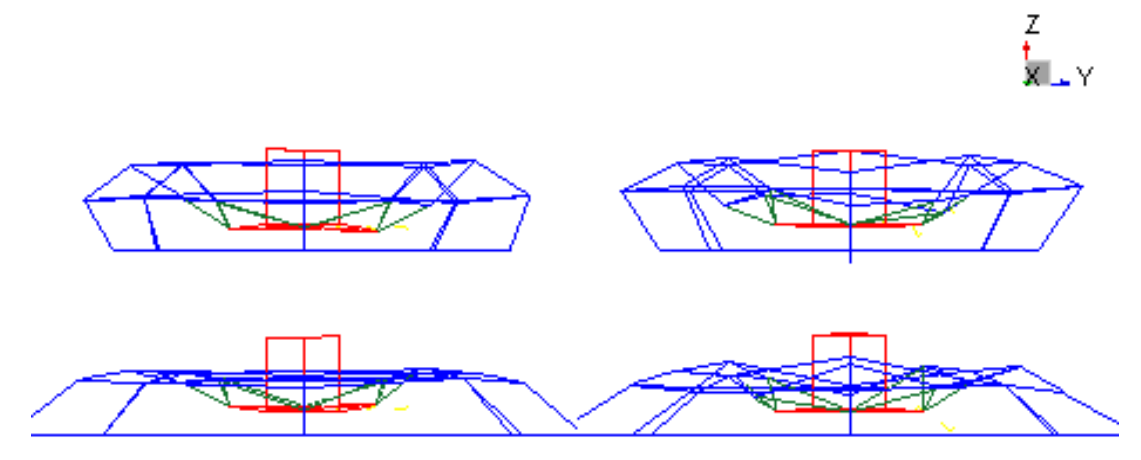

Mode $5: 5509.1946 \mathrm{~Hz}, 0.07 \%$

Mode $7: 4245.2956 \mathrm{~Hz}, 0.61 \%$

Figure B.5: Shell breathing mode shape of turbine in air (Left column) and in ATF (Right column) shown at the opposite motion extremes (Top and bottom). $\mathrm{MAC}=\mathbf{7 2} \%$
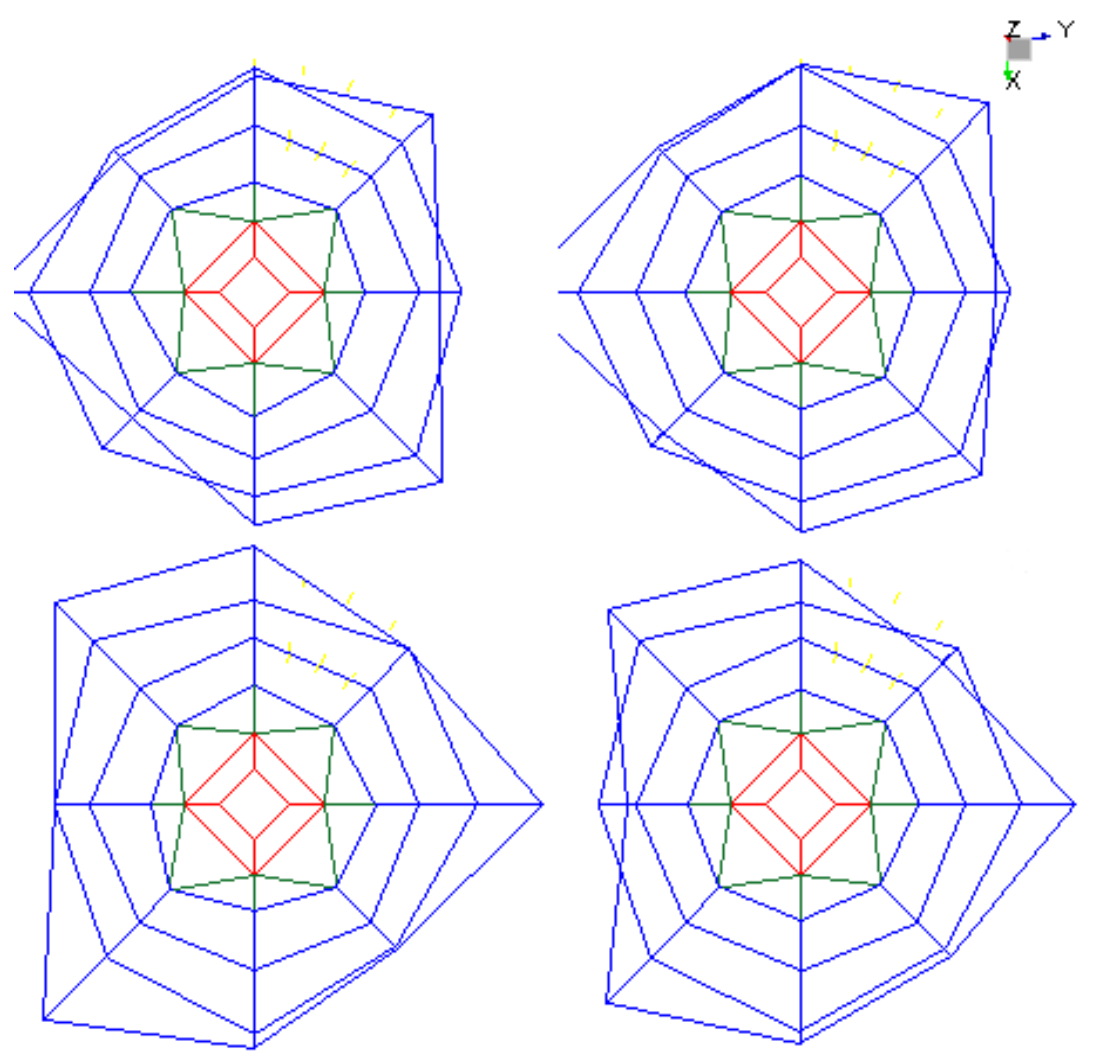

Mode $1: 5414.2871 \mathrm{~Hz}, 0.08 \%$

Mode 5 : $4091.4884 \mathrm{~Hz}, 0.34 \%$

Figure B.6: Three lobed mode shape of turbine in air (Left column) and in ATF (Right column) shown at the opposite motion extremes (Top and bottom). MAC $=69 \%$ 
Typically a MAC of greater than $95 \%$ is needed to indicate that two modes are in fact identical. However, the structure was modified greatly through being submerged into ATF, which will alter the mass loading of the blades and shell, but only slightly change the motion of the hub. The ATF will also change the overall damping characteristic of the structure. Because of this, a MAC of $69 \%$ for the $4091 \mathrm{~Hz}$ to $5414 \mathrm{~Hz}$ mode and $72 \%$ for the $4245 \mathrm{~Hz}$ to $5509 \mathrm{~Hz}$ mode were acceptable to determine that the mode shapes are identical. This means that the motion of the turbine while submerged in ATF at $4 \mathrm{kHz}$ is not purely due to a natural frequency of the individual turbine blades (which were shown in air to be about $8 \mathrm{kHz}$ in Figure B.1), but instead is a vibration mode that encompasses the entire turbine. This case would be much better at producing an excitation that could be detected acoustically. Both of these sets of natural frequencies can be seen in Figure B.4 and Figure B.2.

Both sets of these mode shapes move a great deal of fluid, and are therefore ideal for radiating acoustic energy. Also, although difficult to tell from the static figures, both sets of these modes show a good deal of motion on the trailing edge of the turbine blade. This means that an excitation in the frequency range of 4 to $4.5 \mathrm{kHz}$ on the trailing edge of the turbine blades should be able to make the shell of the turbine move as shown in Figure B.5 and Figure B.6. This means that natural frequencies of the turbine that are excited by the trailing edge of the blades while submerged in the ATF line up with the noise being excited by the turbine during operational testing. Therefore, vortex shedding is a definite possibility as a root cause. 


\section{Appendix C: Copyright Information}

This appendix contains permission letters from Noctua Fan Company, Krieger Publishing, IR Telemetrics, and Darrell Robinette for use of images used in this dissertation.

This letter is permission to use Figures 1.16, 1.17, and 4.7.

Jakob Dellinger - Noctua.at presse@noctua.at

$8 / 2 / 11$

to cmwalber@mtu.edu

Dear Chad,

We hereby grant you permission to use the linked images for your Dissertation. Just out of curiosity, may I ask what you're writing about?

Best Regards, Jakob Dellinger

presse@noctua.at

http://www.noctua.at

RASCOM Computerdistribution Ges.m.b.H

Huttengasse 71-75

A-1160 Wien

Austria

http://www.rascom.at

FN 198653n | Handelsgericht Wien

This letter is permission to use Figures 1.13, 1.15, and 4.5.

KRIEGER PUBLISHING admin@krieger-publishing.com

$8 / 4 / 11$

to cmwalber@mtu.edu

Dear Mr. Walber:

Book: Flow-Induced Vibration

Author: Blevins

ISBN: $1-57524-183-8$

Figures: $3.6,3.14,3.23,3.25$

Copies: for dissertation only 
Permission to duplicate the above material as stated in your email of August 1 is granted with a minimum royalty/permissions fee of $\$ 25.00$. The material is not to be used commercially in any way. Any further permissions must be granted by the publisher only.

Be sure to give proper credit citing author, title, and publisher.

When sending payment include a copy of this email which grants you permission. This will ensure that your account is properly credited. If the material is not used we ask that notification be given so that we may update our records.

Sincerely,

Shannon L. Ryder

Permissions Department

Krieger Publishing Company

1725 Krieger Drive

Malabar, FL 32950 USA

Tel: (321) 724-9542

Fax: (321) 951-3671

Toll Free Within U.S. (800) 724-0025

E-Mail: info@krieger-publishing.com

WEB: www.krieger-publishing.com

This letter is permission to use Figures 1.8, 1.9, and 1.10.

Glen Barna irtel@irtelemetrics.com

$6 / 22 / 11$

to cmwalber@mtu.edu

Chad,

I would say that it IS basic radio frequency transmission ....... in the microwave range......

"Any resistive, piezoresistive, or voltage producing ( thermocouples) transducer......"

We use a "Double FM" technique. You can use the signal evolution diagram to show this.

If you want to come over I can give you more detail for inclusion in your thesis.

You may also use the other torque converter image from our website, as discussed.

Glen Barna 
IR Telemetrics

9064820583

This letter is permission to use Figures $1.1,1.2,1.4,1.6,1.7,2.1,2.2,2.3,2.6,4.1$, and 4.13.

Darrell Robinette darrell.robinette@gm.com

$8 / 26 / 12$

to cmwalber@mtu.edu

Chad,

I have no issues with your request to use the images listed below.

Good luck with finishing up the dissertation. Let me know if you need further input,

Darrell

GM Powertrain

2488309927

Figure 1.4 is a data plot that originated from Strasberg, et. al., "Propeller Cavitation Noise After 35 Years of Study," Noise and Fluids Engineering, 1977. and was used in Darrell Robinette's Dissertation.

Figures 1.12 and 4.4 (Same) are an animation from Wikipedia. As per the following webpage, the copyright holder allows this image to be used for any purpose.

http://en.wikipedia.org/wiki/File:Vortex-street-animation.gif

Publishing agreement for Chapter 2 according to International Journal of Rotating Machinery:

http://www.hindawi.com/journals/ijrm/guidelines/

Papers must be submitted on the understanding that they have not been published elsewhere (except in the form of an abstract or as part of a published lecture, review, or thesis) and are not currently under consideration by another journal published by Hindawi or any other publisher. The submitting author is responsible for ensuring that the article's publication has been approved by all the other coauthors. It is also the authors' responsibility to ensure that the articles emanating from a particular institution are submitted with the approval of the necessary institution. Only an acknowledgment from the editorial office officially establishes the date of receipt. Further correspondence and proofs will be sent to the author(s) before publication unless otherwise indicated. It is a condition of submission of a paper that the authors permit editing of the paper for 
readability. All enquiries concerning the publication of accepted papers should be addressed to ijrm@hindawi.com.

Publishing agreement for Chapter 4 according to Society of Experimental Mechanics: Experimental Techniques:

http://mc.manuscriptcentral.com/societyimages/ext/CTA_ET_NEW_Mar-2011.PDF

2. Reproduction, posting, transmission or other distribution or use of the final Contribution in whole or in part in any medium by the Contributor as permitted by this Agreement requires a citation to the Journal and an appropriate credit to the Society and Wiley-Blackwell as Publisher, suitable in form and content as follows: (Title of Article, Contributor, Journal Title and Volume/Issue, Copyright $\mathbb{C}$ [year], [copyright owner as specified in the Journal] [Publisher]. Links to the final article on Wiley-Blackwell's website are encouraged where appropriate.

Publishing agreement for Chapter 3 according to SAE NVH

http://volunteers.sae.org/authors/copyright.pdf

SAE hereby grants Assignor a nonexclusive license to reproduce and publicly distribute the Work for five (5) years following the sixth (6th) month after the date of first publication of the Work by SAE. Any such reproduction or distribution of the Work shall include the SAE copyright notice thereon and shall be offered free of charge and without implying endorsement by SAE of any service or product. 